\title{
ChARACTERIZATION OF BLOOD-BRAIN BARRIER DISRUPTION IN A FOCAL MODEL OF NEUROMYELITIS OPTICA
}

\author{
Doctoral Thesis \\ In partial fulfillment of the requirements for the degree \\ "Doctor rerum naturalium (Dr. rer. nat.)" \\ in the Molecular Medicine Study Program \\ at the Georg-August University Göttingen
}

submitted by

Anne Winkler

born in Ilmenau

Göttingen, June 2015 


\section{Members of the Thesis Committee:}

\section{Supervisor (Reviewer)}

Prof. Dr. Wolfgang Brück

Department of Neuropathology

University Medical Center, Georg-August-University Göttingen

\section{Second member of the thesis committee (Reviewer)}

Prof. Dr. Mikael Simons

Department of Cellular Neuroscience

Max-Planck-Institute for Experimental Medicine, Göttingen

Department of Neurology

University Medical Center, Georg-August-University Göttingen

Third member of the thesis committee

Prof. Dr. Alexander Flügel

Institute for Multiple Sclerosis Research, Dept. of Neuroimmunology

University Medical Center, Georg-August-University Göttingen

Date of Disputation: 


\section{AfFIDAVIT}

Here I declare that my doctoral thesis entitled "Characterization of blood-brain barrier disruption in a focal model of neuromyelitis optica" has been written independently with no other sources and aids than quoted.

Anne Winkler

Göttingen, June 2015 
Meinen Eltern 


\section{List of PUbLications}

\section{Original article}

Kinzel, S., Lehmann-Horn, K., Zamvil, S.S., Winkler, A., Bernard, C.C., Stadelmann-Nessler, C., Brück, W., and Weber, M.S. (2015). Myelin-specific antibodies trigger spontaneous CNS autoimmune disease in the absence of myelin-specific $B$ cells. Journal of Neuroimmunology $275,7-8$.

Li, H.*, Moll, J.*, Winkler, A*., Frappart, L., Brunet, S., Hamann, J., Kroll, T., Verlhac, M.H., Heuer, H., Herrlich, P., Ploubidou, A. (2015). RHAMM deficiency disrupts folliculogenesis resulting in female hypofertility. Biol Open.

$*$ = equal contribution

Wrzos, C., Winkler, A., Metz, I., Kayser, D.M., Thal, D.R., Wegner, C., Brück, W., Nessler, S., Bennett, J.L., and Stadelmann, C. (2014). Early loss of oligodendrocytes in human and experimental neuromyelitis optica lesions. Acta Neuropathol 127, 523-538.

\section{Abstract}

Winkler, A., Wrzos, C., Brück, W., Bennett, J.L., Nessler, S., Stadelmann, C.

Early breakdown of the blood-brain barrier in a model of neuromyelitis optica $12^{\text {th }}$ Congress of the International Society of Neuroimmunology, November $9^{\text {th }}-13^{\text {th }} 2014$, Mainz, Germany, Postersession

Winkler, A., Wrzos, C., Brück, W., Bennett, J.L., Nessler, S., Stadelmann, C. Early breakdown of the blood-brain barrier in a model of neuromyelitis optica $16^{\text {th }}$ International Symposium on "Signalling in the Blood-Brain Barriers", September $12^{\text {th }}-$ $14^{\text {th }} 2013$, Sümeg, Hungary, Postersession 


\section{TABLE OF CONTENTS}

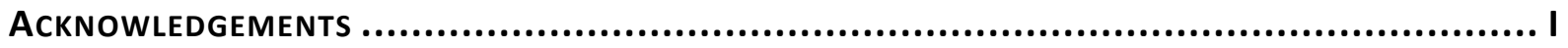

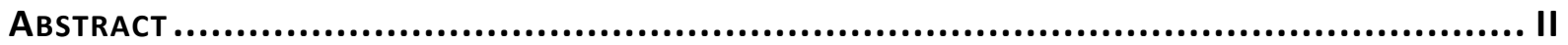

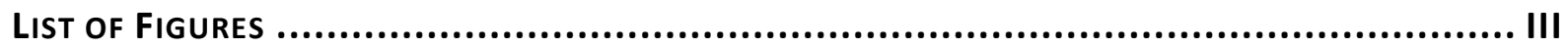

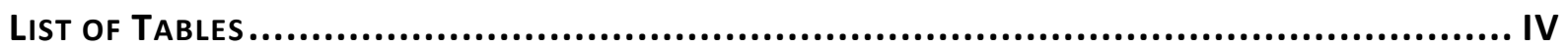

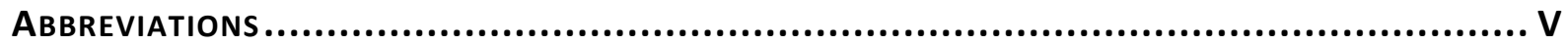

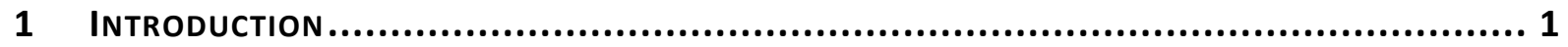

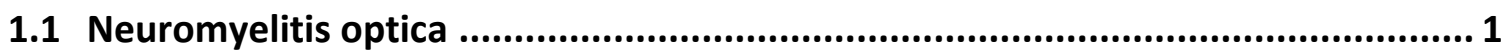

1.1.1 Clinical presentation and disease course ................................................. 1

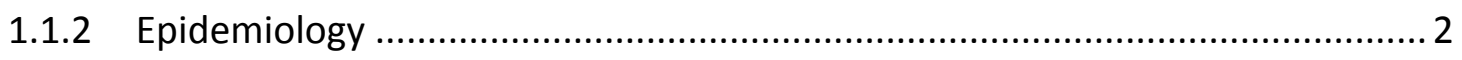

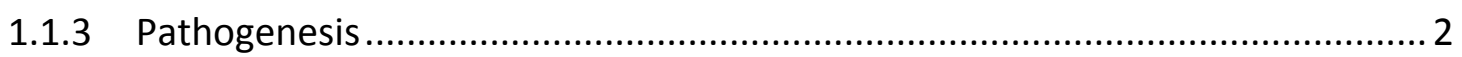

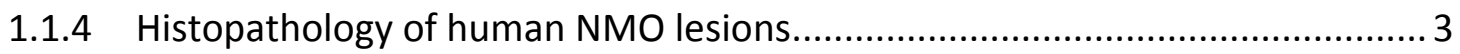

1.1.5 The blood-brain barrier in NMO ........................................................... 4

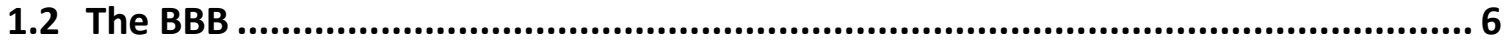

1.2.1 The BBB phenotype of brain endothelial cells............................................ 6

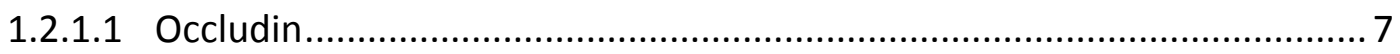

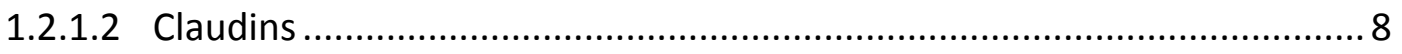

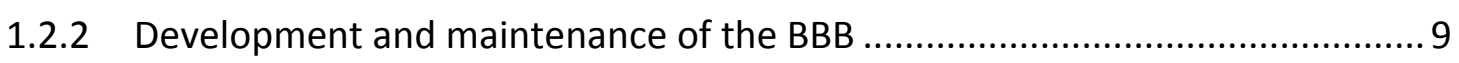

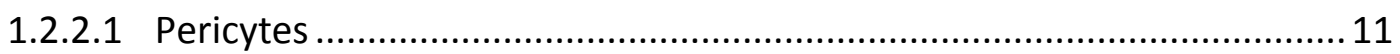

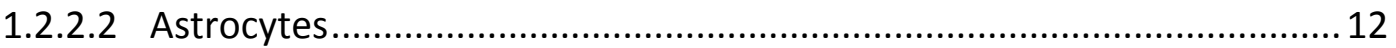

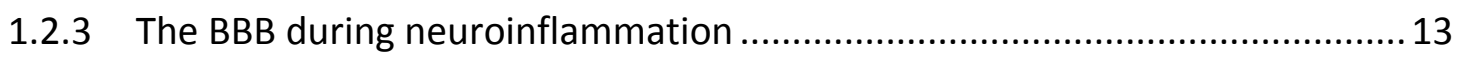

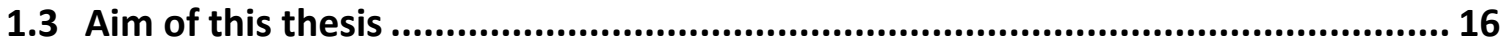

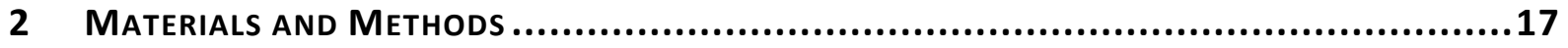

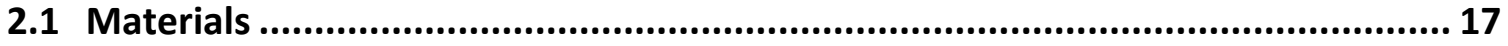

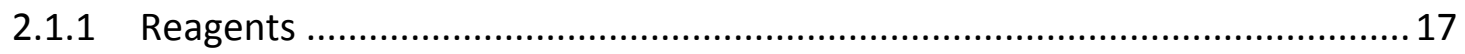

2.1.2 Solutions, buffers and cell culture media ................................................... 19

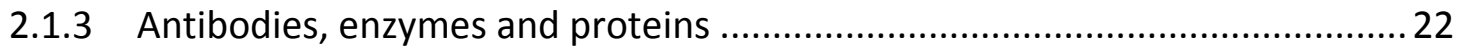

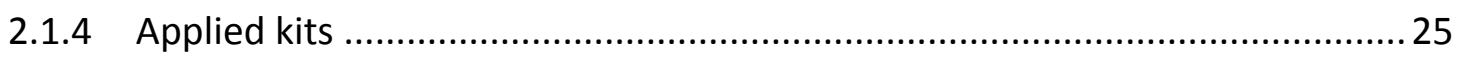

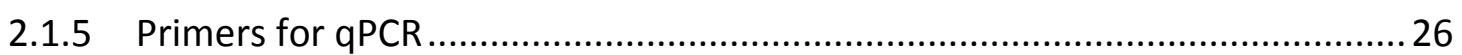

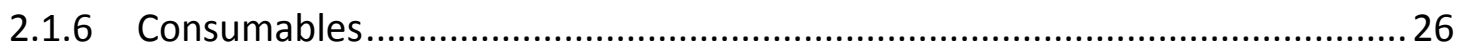

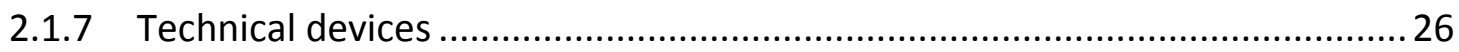

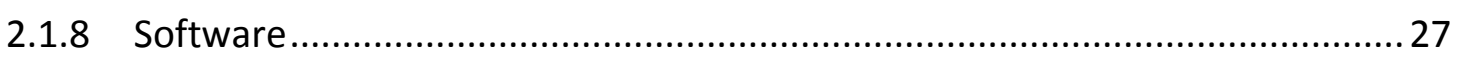

2.2 Human neuromyelitis optica (NMO) tissue .................................................. 27

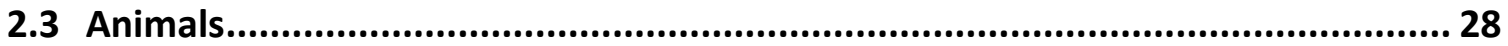

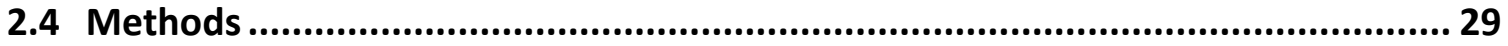




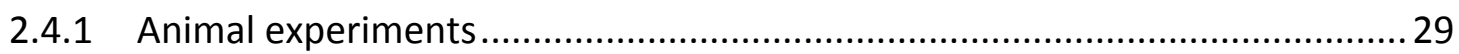

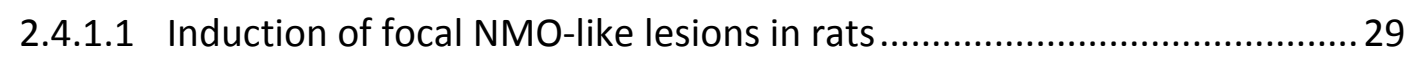

2.4.1.2 Assessment of blood brain barrier (BBB) permeability ..........................2 29

2.4.1.3 Depletion of polymorphonuclear cells (PMN) in rats .............................30

2.4.1.4 Pharmacological inhibition of the complement component 5a receptor

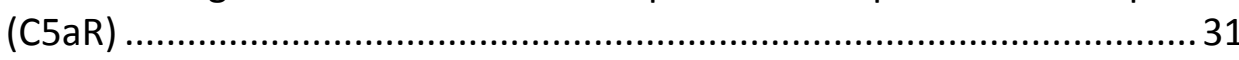

2.4.1.5 Pharmacological inhibition of neutrophil elastase ..............................31

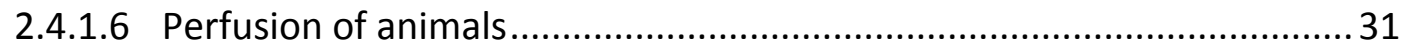

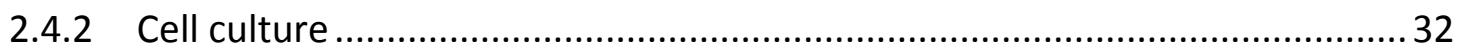

2.4.2.1 Isolation and purification of primary rat brain endothelial cells (RBEC) and measurement of the transendothelial resistance (TEER) .................32

2.4.2.2 Isolation and purification of primary rat astrocytes ..............................33

2.4.2.3 Co-culture of RBEC and astrocytes as in vitro model of the BBB..............33

2.4.2.4 Incubation of RBEC monolayers with proteases and assessment of

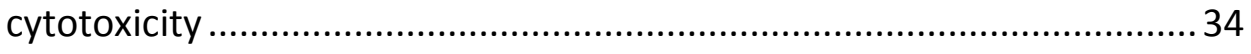

2.4.2.5 Immunocytochemcial staining of RBEC .............................................. 34

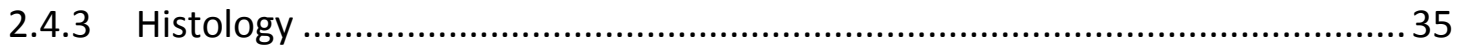

2.4.3.1 Cutting, deparaffinization and dehydration of histological paraffin-

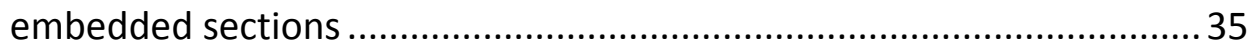

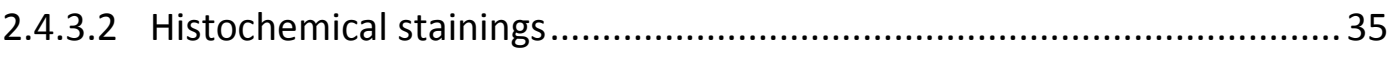

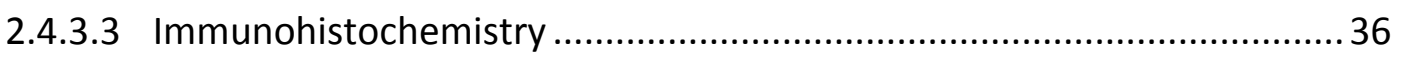

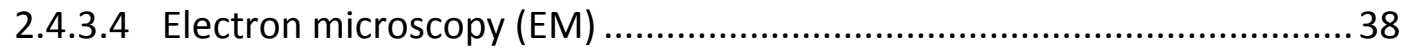

2.4.4 Microdissection of lesions for mRNA expression analysis.............................. 39

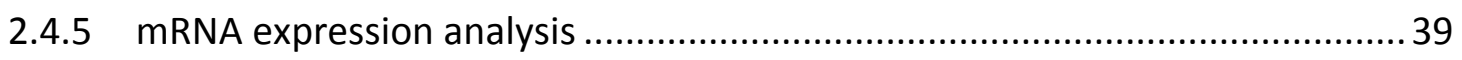

2.4.6 Morphometric analysis and data acquisition ..............................................39

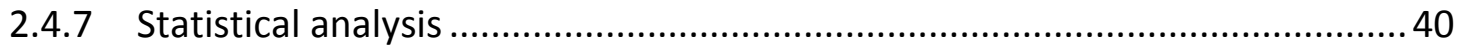

3 Results

3.1 Induction of neuromyelitis optica (NMO)-like lesions results in breakdown of the blood-brain barrier (BBB).

3.1.1 Time course of astrocyte depletion after focal injection of NMO-antibody and human complement .....

3.1.2 Breakdown of the BBB is observed $6 \mathrm{~h}$ after lesion induction ....................... 42

3.1.3 Injection of tracer molecules indicates rapid restoration of the BBB within 24 $\mathrm{h}$ after lesion induction.

3.2 Co-culture of rat brain endothelial cells (RBEC) with astrocytes as an in-vitro model of the BBB did not result in increased TEER values.

3.3 Platelet-derived growth factor receptor $\beta$ (PDGFRß) positive cells are in close proximity to brain vessels $6 \mathrm{~h}$ and $24 \mathrm{~h}$ after lesion induction......................... 47

3.4 Characterization of tight junctions (TJ) in NMO-like lesions 
3.4.1 Transient loss of occludin from TJ after lesion induction ...............................49

3.4.2 No morphological alterations of TJ on the ultrastructural level .....................52

3.4.3 mRNA levels of occludin and claudin 5 are increased in NMO-like lesions ....53

3.5 Infiltration of polymorphonuclear cells (PMN) correlates with FITC-albumin extravasation $6 \mathrm{~h}$ after lesion induction ............................................................ 54

3.6 Effect of PMN depletion on lesion development...............................................57

3.6.1 Depletion of PMN prevents astrocyte depletion, breakdown of the BBB and occludin loss $6 \mathrm{~h}$ after lesion induction

3.6.2 Treatment with anti-PMN antiserum decreases immune cell numbers in the blood

3.7 Inhibition of complement component $\mathrm{C5a}$ receptor (C5aR) significantly reduces astrocyte lesion size $6 \mathrm{~h}$ after lesion induction

3.8 Investigation of PMN granule proteases in the regulation of BBB permeability .. 64

3.8.1 Treatment of RBEC with MMP9 decreases electrical resistance in vitro ........64

3.8.2 MMP9 positive PMN are abundant in NMO-like lesions $6 \mathrm{~h}$ after lesion induction

3.8.3 Inhibition of NE reduces astrocyte lesion size and PMN infiltration $6 \mathrm{~h}$ after lesion induction.

3.9 PMNs are a component of the inflammatory infiltrate in early human NMO lesions

Discussion.

4.1 Rapid restoration of the BBB in the focal NMO model, independent of loss of astrocytes and occludin

4.2 PMN play a crucial role in the breakdown of the BBB in focal NMO like lesions.. 76

4.3 PMN attracting molecules and released granule contents modulate NMO-like lesion development and BBB permeability

5 Summary ANd CONCLUSIONS 


\section{ACKNOWLEDGEMENTS}

First and foremost, I would like to thank my supervisor Prof. Dr. Christine StadelmannNessler not only for her excellent guidance and inspiring scientific discussions throughout my PhD project but also for giving me the possibility to develop ideas on my own. Also I would like to thank her for her personal support and her patience and for always taking time for questions and problems.

I am also grateful to Prof. Dr. Wolfgang Brück for giving me the opportunity to join his Department and for the scientific discussions.

I want to thank my thesis committee members Prof. Dr. Alexander Flügel and Prof. Dr. Mikael Simons for their interest in my project and their supportive contributions during my progress reports. Additionally I want to thank Prof. Dr. Mikael Simons for agreeing to act as second reviewer.

Furthermore, I want to thank Dr. Claudia Wrzos for the methods she taught me and the scientific discussions I had with her; as well as Dr. Stefan Nessler for his scientific advice.

Many thanks to our laboratory assistants Brigitte, Katja, Heidi, Olga, Angela and Jasmin for their excellent technical and theoretical support in histology, immunohistochemistry and electron microscopy as well as for the nice conversations and the enjoyable atmosphere in the lab. I am also grateful to Michael Haberl for his help with the FACS staining and analysis of blood samples. I want to thank Prof. Dr. Jeffrey Bennett for providing me with his recombinant antibodies. Also, I want to thank Nadine, Silke and David for their helpful comments on this manuscript.

I am grateful to Nielsen, Silke, Claudia, Nadine, Erika, Verena, Franziska, Alonso, Patrik, Lena, Linda, and all other colleagues and friends for the enjoyable working atmosphere, scientific discussions, and also the nice time we spent outside the lab.

I want to acknowledge our secretaries Cynthia and Heidi for administrative support and the Molecular Medicine PhD program, especially Dr. Eric Meskauskas.

I am very grateful to my family, especially my parents Wolfgang and Rita and my brother Paul for supporting me over all these years. Thank you for your unlimited love and persistent confidence in me.

Finally, I would like to thank David for believing in me. 


\section{Abstract}

Neuromyelitis optica (NMO) is a demyelinating autoimmune disease of the central nervous system (CNS), with serum anti-aquaporin4 (AQP4) antibodies (Ab) detected in the majority of patients. Binding of NMO-Ab to AQP4 results in complement- and cell-mediated astrocyte depletion. In addition, a severe impairment of the blood-brain barrier (BBB) is observed as evidenced by gadolinium-enhanced lesions on magnetic resonance imaging (MRI). To investigate the structural, molecular and cellular correlates of the BBB breakdown, we employed a focal model of NMO-like lesions in rats which is based on the intracerebral injection of a human recombinant NMO-Ab directed against AQP4, and human complement.

Astrocytes and pericytes are considered crucial for maintenance and repair of the BBB. In NMO-like lesions, we demonstrated that a transient breakdown of the BBB coincided with the onset of astrocyte loss. However, the BBB integrity to vascular tracers was rapidly restored, even in the absence of astrocytes. No loss of pericytes from NMO-like lesions was observed indicating that pericytes might contribute to the rapid restoration of the BBB.

Tight junctions (TJ) restrict the paracellular diffusion of solutes across the BBB. Therefore, we analyzed the TJ in NMO-like lesions and detected a transient loss of the TJ protein occludin, while the expression of claudin-3 and claudin-5 was not altered. However, no morphological alterations of the TJ were observed on the ultrastructural level, and the integrity of the BBB to vascular tracers was re-established in the absence of occludin.

Inflammatory cells infiltrating the CNS were shown to contribute to BBB disruption. In early NMO-like lesions we demonstrated that polymorphonuclear cells (PMN) were the most abundant infiltrating leukocytes and that the number of PMN in the lesions correlated with the extent of vascular tracer extravasation. Depletion of PMN prevented not only the breakdown of the BBB, but interestingly also the loss of astrocytes. Furthermore, we could show that inhibition of the complement component C5a receptor (C5aR), which is important for PMN attraction and activation, reduced the area of astrocyte loss in NMO-like lesions. In addition, proteases released from PMN granules upon activation were implied in BBB disruption. We demonstrated that matrix metalloproteinase 9 (MMP9) decreased the electrical resistance of endothelial monolayers and that inhibition of the neutrophil elastase (NE), another PMN protease, reduced the loss of astrocytes and PMN infiltration in vivo.

This study affirms the importance of PMN in the development of NMO-like lesions and is the first to show that infiltrating PMN mediate the breakdown of the BBB. These findings, together with the detection of PMN in early human NMO lesions, identify the PMN mediated breakdown of the BBB as a promising target for future therapeutic approaches. 


\section{LIST OF FIGURES}

Figure 1: Early NMO lesions are characterized by loss of GFAP and AQP4 immunoreactivity, demyelination, relative axonal preservation and breakdown of the BBB

Figure 2: Cellular and structural components of the neurovascular unit ..... 10

Figure 3: Injection of NMO-Ab and human complement resulted in astrocyte loss

Figure 4: Breakdown of the BBB was detected $6 \mathrm{~h}$ after focal injection as assessed by immunohistochemistry for IgG and fibrinogen

Figure 5: Restoration of the BBB within $24 \mathrm{~h}$ of focal intracerebral injection of NMO-Ab and human complement

Figure 6: Co-culture of RBEC with astrocytes as an in vitro model of the BBB did not result in altered TEER values.

Figure 7: No loss of PDGFRß positive cells from the blood vessels in NMO-like lesions $6 \mathrm{~h}$ and $24 \mathrm{~h}$ after focal NMO-Ab injection

Figure 8: Loss of occludin from the blood vessels was observed in focal NMO-like lesions 51

Figure 9: Ultrastructure of TJ in cerebral blood vessels after lesion induction 52

Figure 10: mRNA expression of occludin and claudin-5 were increased $10 \mathrm{~h}$ and $24 \mathrm{~h}$ after stereotactic injection

Figure 11: Strong infiltration of PMN correlated with extravasation of FITC-albumin $6 \mathrm{~h}$ after lesion induction

Figure 12: Depletion of PMN inhibited astrocyte lesion formation, breakdown of the BBB and loss of occludin form the TJ $6 \mathrm{~h}$ after lesion induction.

Figure 13: Treatment of animals with anti-PMN antiserum resulted in a strong decrease of PMN numbers but also a non-selective reduction of leukocytes compared to normal serum treated animals

Figure 14: Decreased astrocyte loss after systemic treatment with the C5aR antagonist PMX-53 $6 \mathrm{~h}$ after lesion induction

Figure 15: Treatment of RBEC with MMP9, but not NE or cathepsin G, reduced TEER in vitro 65

Figure 16: Intracellular MMP9 expression was detected in infiltrating PMN $6 \mathrm{~h}$ and $12 \mathrm{~h}$ after lesion induction

Figure 17: Inhibition of NE using Sivelestat resulted in a significant reduction of astrocyte lesion size and PMN infiltration $6 \mathrm{~h}$ after lesion induction

Figure 18: In the inflammatory infiltrate of early human NMO lesions PMN, monocytes, 


\section{LIST OF TABLES}

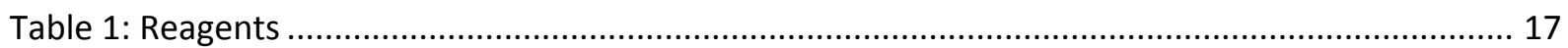

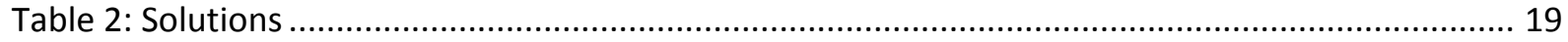

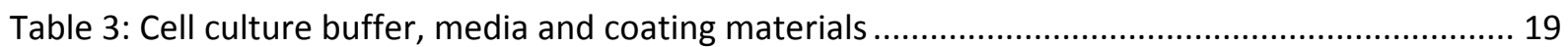

Table 4: Solutions for histochemistry, immunohistochemistry and electron microscopy ................. 21

Table 5: Primary antibodies for immunohistochemical staining .................................................. 22

Table 6: Secondary antibodies for immunohistochemical staining .......................................... 23

Table 7: Monoclonal antibodies for flow cytometry............................................................ 24

Table 8: Recombinant human antibodies for induction of focal NMO lesions in vivo......................... 24

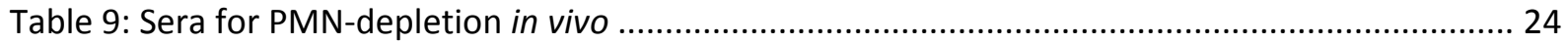

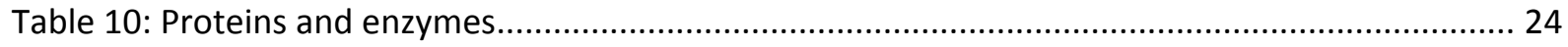

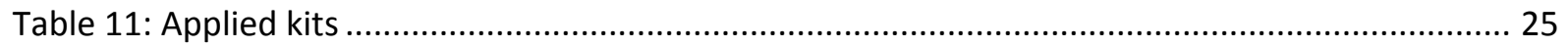

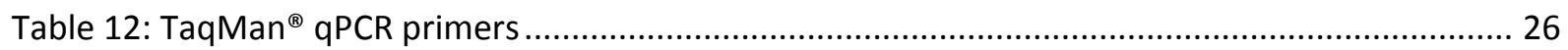

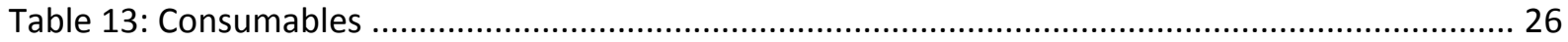

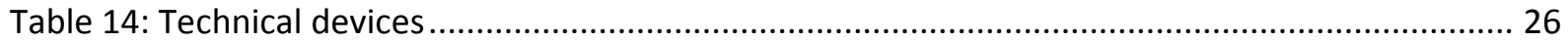

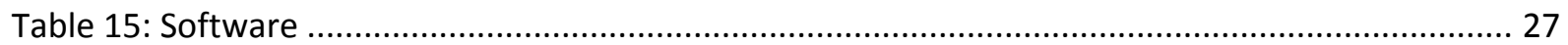

Table 16: Clinical data of patients with anti-AQP4 seropositive NMO or NMO spectrum disorder ..... 28 


\section{AbBreviations}

$A b$

$A B C$ transporter

ADCC

AGT

AGT-II

AJ

Ang-1

ApoE

AQP4

AT1

BBB

${ }^{\circ} \mathrm{C}$

C5aR

C5L2

CAE

$\mathrm{Ccl}$

$\mathrm{Ccr}$

CD

CDC

CNS

CSF

ctrl

CXCL

Cy5

Da

DAB

DAMP

DAPI

DDSA

DMP-30

DMSO
Antibody/antibodies

ATP-binding cassette transporter

Antibody-dependent cellular cytotoxicity

Angiotensinogen

Angiotensin II

Adherens junction

Angiopoietin 1

Apolipoprotein E

Aquaporin 4

Angiotensin-II receptor type 1

Blood brain barrier

Degrees Celsius

Complement component $\mathrm{C} 5 \mathrm{a}$ receptor

C5a receptor-like 2

Chloroacetate esterase

Chemokine ( $\mathrm{C}-\mathrm{C}$ motif) ligand

C-C chemokine receptor

Cluster of differentiation

Complement dependent cytotoxicity

Central nervous system

Cerebrospinal fluid

Control

Chemokine (C-X-C motif) ligand

Cyanine 5

Dalton

3,3'-Diaminobenzidine

Damage-associated molecular pattern molecules

4',6-diamidino-2-phenylindole

2-Dodecenylsuccinic acid anhydride

2,4,6 Tris(dimethylaminomethyl)phenol

Dimethyl sulfoxide 
e.g.

EAAT2

EAE

EC

EDSS

EM

ESAM

FACS

FCS

FITC

g

GFAP

Glut1

GM

h

$\mathrm{H}_{2} \mathrm{O}_{2}$

$\mathrm{HCl}$

i.e.

i.p.

i.v.

ICAM-1

iDTR

Ig

IL

INF

JAM

$\mathrm{kDa}$

I

LAM 1

LCM

LPS

M

MARVEL

MBP
Exempli gratia

Excitatory amino acid transporter-2

Experimental autoimmune encephalomyelitis

Endothelial cell(s)

Expanded disability status scale

Electron microscopy

Endothelial cell-selective adhesion molecule

Fluorescence activated cell sorting

Fetal calf serum

Fluorescein isothiocyanate

Gram

Glial fibrillary acidic protein

Glucose transporter 1

Grey matter

Hour(s)

Hydroxic peroxide

Hydrochloric acid

Id est

Intraperitoneal

Intravenous

Intercellular adhesion molecule 1

Cre-inducible diphtheria toxin receptor

Immunoglobulin

Interleukin

Interferon

Junctional adhesion molecule

Kilodalton

Liter

Laminin $\gamma 1$

Laser capture microdissection

Lipopolysaccharide

Molar

MAL and related proteins for vesicle trafficking and membrane link Myelin basic protein 


\begin{tabular}{|c|c|}
\hline$\mu g$ & Microgram \\
\hline mg & Milligram \\
\hline $\min$ & Minute(s) \\
\hline$\mu l$ & Microliter \\
\hline $\mathrm{ml}$ & Milliliter \\
\hline$\mu \mathrm{m}$ & Micrometer \\
\hline$\mu \mathrm{M}$ & Micromolar \\
\hline MMP9 & Matrix metalloproteinase 9 \\
\hline MRI & Magnetic resonance imaging \\
\hline MRP14 & Myeloid-related protein-14 \\
\hline MS & Multiple sclerosis \\
\hline $\mathrm{NaOH}$ & Sodium hydroxide \\
\hline NE & Neutrophil elastase \\
\hline $\mathrm{nm}$ & Nanometer \\
\hline NMO & Neuromyelitis optica \\
\hline NVU & Neurovascular unit \\
\hline$P$ & Postnatal day \\
\hline PAMP & Pathogen-associated molecular pattern molecules \\
\hline PBS & Phosphate buffered saline \\
\hline PDGF & Platelet-derived growth factor \\
\hline PDGFRß & Platelet-derived growth factor receptor $ß$ \\
\hline PE & Phycoerythrin \\
\hline PerCP & Peridinin chlorophyll protein \\
\hline PFA & Paraformaldehyde \\
\hline PKC & Protein kinase C subtype \\
\hline PLL & Poly-L-Lysin \\
\hline PMN & Polymorphonuclear cell(s) \\
\hline POX & Streptavidin-horseradish peroxidase \\
\hline RBEC & Rat brain endothelial cell(s) \\
\hline $\mathrm{RR}$ & Relapsing remitting \\
\hline RT & Room temperature \\
\hline $\mathrm{s}$ & Second(s) \\
\hline S100ß & S100 calcium-binding protein $ß$ \\
\hline SD & Standard deviation \\
\hline
\end{tabular}


Shh

SSeCKS

TBE

TCR

TEER

TGF

TJ

TNF

Tris

U

VCAM-1

VEGF

VWF

WBC

WM

wt

ZO

ZONAB
Sonic hedgehog

Src-suppressed C-kinase substrate

Tris/borate/EDTA

T cell receptor

Transendothelial electrical resistance

Transforming growth factor

Tight junction(s)

Tumor necrosis factor

Tris(hydroxymethyl)aminomethane

Units

Vascular cell adhesion molecule 1

Vascular endothelial growth factor

von Willebrand Factor

White blood cells

White matter

Wild type

Zonula occludens

ZO-1-associated nucleic acid binding protein 


\section{$1 \quad$ INTRODUCTION}

\section{$1.1 \quad$ Neuromyelitis optica}

Neuromyelitis optica (NMO) is an idiopathic demyelinating disease of the central nervous system (CNS). It is also known as Devic's disease, named after the French neurologist Eugène Devic. Devic and his student Gault established the syndrome with the characteristics of acute myelitis and optic neuritis in 1894 (Devic, 1894; Gault, 1894). Whether NMO represents a variant of multiple sclerosis (MS) was discussed for a long time since NMO and MS both share the features of inflammatory demyelination, optic neuritis and myelitis. However, today NMO is recognized as a separate disease entity with specific clinical and pathological features. The discovery of a specific NMO-antibody (Ab) in 2004 by Lennon and coworkers contributed substantially to the understanding of the pathogenesis of NMO (Lennon et al., 2004; Lennon, 2005).

\subsubsection{Clinical presentation and disease course}

Characteristic features of NMO are severe attacks of optic neuritis and longitudinally extensive transverse myelitis. Frequently, attacks of optic neuritis and myelitis occur sequentially with separating periods spanning sometimes years or decades (Wingerchuk et al., 1999). Patients typically present with loss of vision, severe symmetric paraplegia, sensory disturbances and loss of bladder control (Wingerchuk et al., 2007). Cervical spinal cord lesions may extend into the brain stem, resulting in symptoms like vomiting, nausea and intractable hiccups (Almekhlafi et al., 2011; Misu et al., 2005; Wingerchuk et al., 1999). Although brain lesions were historically viewed as an exclusion criterion for the diagnosis of NMO (Wingerchuk et al., 1999), brain abnormalities detected by magnetic resonance imaging (MRI) are described in about $50-65 \%$ of NMO patients which are normally clinically silent. About $10 \%$ of the patients develop unique NMO brain lesions in the hypothalamus, corpus callosum, brainstem or periventricular (Bichuetti et al., 2008; Cabrera-Gomez et al., 2007; Pittock et al., 2006). The revised criteria for NMO include acute myelitis and optic neuritis as absolute criteria. Furthermore, 2 of the following 3 criteria are required: longitudinally extensive myelitis with lesions spanning typically 3 or more contiguous vertebral segments, negative or MS atypical brain MRI and NMO-IgG seropositivity (Wingerchuk et al., 2007).

A relapsing-remitting (RR) disease course is the most common form in NMO which affects 80 - $90 \%$ of the patients. $10-20 \%$ of the patients develop a monophasic disease course 
(Wingerchuk et al., 1999). The recovery after attacks is incomplete and results in the attackrelated development of permanent disabilities. Disabilities like blindness or paraplegia are often severe and accumulate rapidly (Wingerchuk et al., 1999; Wingerchuk et al., 2007).

\subsubsection{Epidemiology}

NMO more frequently affects females than males with a ratio of 9:1. The median age of onset is in the late thirties, however, NMO can also affect young children or older people (Asgari et al., 2011; Cabre et al., 2001; Cabrera-Gomez et al., 2009; Kuroiwa et al., 1975; Rivera et al., 2008). In one study, e.g., the reported age at disease onset ranged from 3-81 years (Mealy et al., 2012). NMO is a rare disease, and population based studies suggest yearly incidence rates of 0.053 to $0.4 \mathrm{NMO}$ cases per 100,000 persons and prevalence rates of 0.52 to 4.4 per 100,000 (Asgari et al., 2011; Cabre et al., 2001; Cabrera-Gomez et al., 2009; Kuroiwa et al., 1975; Rivera et al., 2008).

\subsubsection{Pathogenesis}

$\mathrm{NMO}$ is a severe demyelinating autoimmune disease with the astrocyte as the main target. About $70 \%$ of the patients are seropositive for specific NMO-Ab directed against aquaporin4 (AQP4), a water channel expressed on astrocytic endfeet (Jarius et al., 2011; Lennon et al., 2005; Lennon et al., 2004; Mealy et al., 2012). Several observations in patients indicate a pathogenic role of NMO-Ab: complement and Ab depositions together with loss of AQP4 immunoreactivity were observed in human NMO lesions (Hinson et al., 2007; Roemer et al., 2007). Furthermore, NMO-Ab levels correlated with disease activity (Jarius et al., 2012a; Kim et al., 2012; Takahashi et al., 2007), and B-cell depletion and plasma exchange are beneficial for NMO patients (Bonnan et al., 2009; Cree et al., 2005; Watanabe et al., 2007). In addition, $\mathrm{NMO}-\mathrm{Ab}$ were shown to be pathogenic in various in vitro and in vivo studies. NMO-Ab induced astrocyte death was demonstrated to be mediated by complement-dependent and cell-mediated cytotoxicity (CDC and ADCC) (Phuan et al., 2012; Ratelade et al., 2013; Vincent et al., 2008; Wrzos et al., 2014; Zhang and Verkman, 2013). Furthermore it was shown in vivo that passive transfer of NMO-Ab into rats with experimental autoimmune encephalomyelitis (EAE), an inflammatory disease commonly used as an experimental model for MS, results in NMO-like lesions in the recipient animals (Bennett et al., 2009; Bradl et al., 2009; Kinoshita et al., 2009; Wrzos et al., 2014). Similarly, development of NMO-like lesions was observed after intracerebral injection of NMO-Ab together with human complement in rats and mice (Ratelade et al., 2013; Saadoun et al., 2010; Wrzos et al., 2014). The resulting NMO-like 
lesions were characterized by loss of astrocytes and oligodendrocytes, demyelination, immune cell infiltration and deposits of complement and immunoglobulin (Ig) G.

With regard to disease effector mechanisms, CDC and ADCC were found to be relevant in experimental models. Furthermore, also in human NMO therapeutic approaches indicate an important role of CDC in the disease process. Using a C1-esterase inhibitor (Cinryze ${ }^{\circledR}$ ) as addon therapy to prevent the activation of the complement cascade after the binding of the NMO-Ab to AQP4 in NMO lesions reduced neurologic damage, and improved outcomes were observed in a phase 1 open-label trial (Levy and Mealy, 2014). Another open-label trial used the monoclonal antibody eculizumab that is directed against the complement component C5 and thereby inhibits the effector pathway of the complement cascade. Treatment of RR NMO patients with eculizumab significantly reduced attack frequency and improved or stabilized the neurological disability measures (Pittock et al., 2013).

\subsubsection{Histopathology of human NMO lesions}

NMO lesions in the CNS are characterized by extensive demyelination and astrocyte loss which may affect both the grey and white matter. Furthermore, demyelination is associated with a pronounced reduction in the numbers of oligodendrocytes (Lucchinetti et al., 2002; Parratt and Prineas, 2010; Wrzos et al., 2014). Early NMO lesions present with relative axonal preservation (Figure $1 \mathrm{~B}$ ), while in more advanced stages of lesion development axonal loss is observed (Lucchinetti et al., 2002; Parratt and Prineas, 2010). Unique for NMO lesions is the extensive loss of astrocytes (Hinson et al., 2007; Roemer et al., 2007). Interestingly, loss of AQP4 immunoreactivity exceeds the loss of glial fibrillary acidic proteins (GFAP) immunoreactivity, an intermediate filament protein used as marker for astrocytes, within NMO lesions (Figure 1 C vs. D; Parratt and Prineas, 2010). Furthermore, perivascular IgG and IgM deposits associated with immunoreactivity for the terminal complement membrane attack complex are observed in a characteristic rim and rosette pattern in active NMO lesions (Lucchinetti et al., 2002). Moreover, focal deposits of Ig and complement coincide with areas of AQP4 loss (Hinson et al., 2007; Roemer et al., 2007). Vascular Ig and fibrinogen deposits (Figure $1 \mathrm{E}$ ) also indicate a loss of blood-brain barrier (BBB) integrity in NMO lesions. In addition, blood vessels are thickened and hyalinized (Lucchinetti et al., 2002; Mandler et al., 1993). The immune cell infiltrate in NMO lesions consists of monocytes, abundant numbers of macrophages, lymphocytes and polymorphonuclear cells (PMN), including neutrophils, basophils and eosinophils (Lucchinetti et al., 2002). 


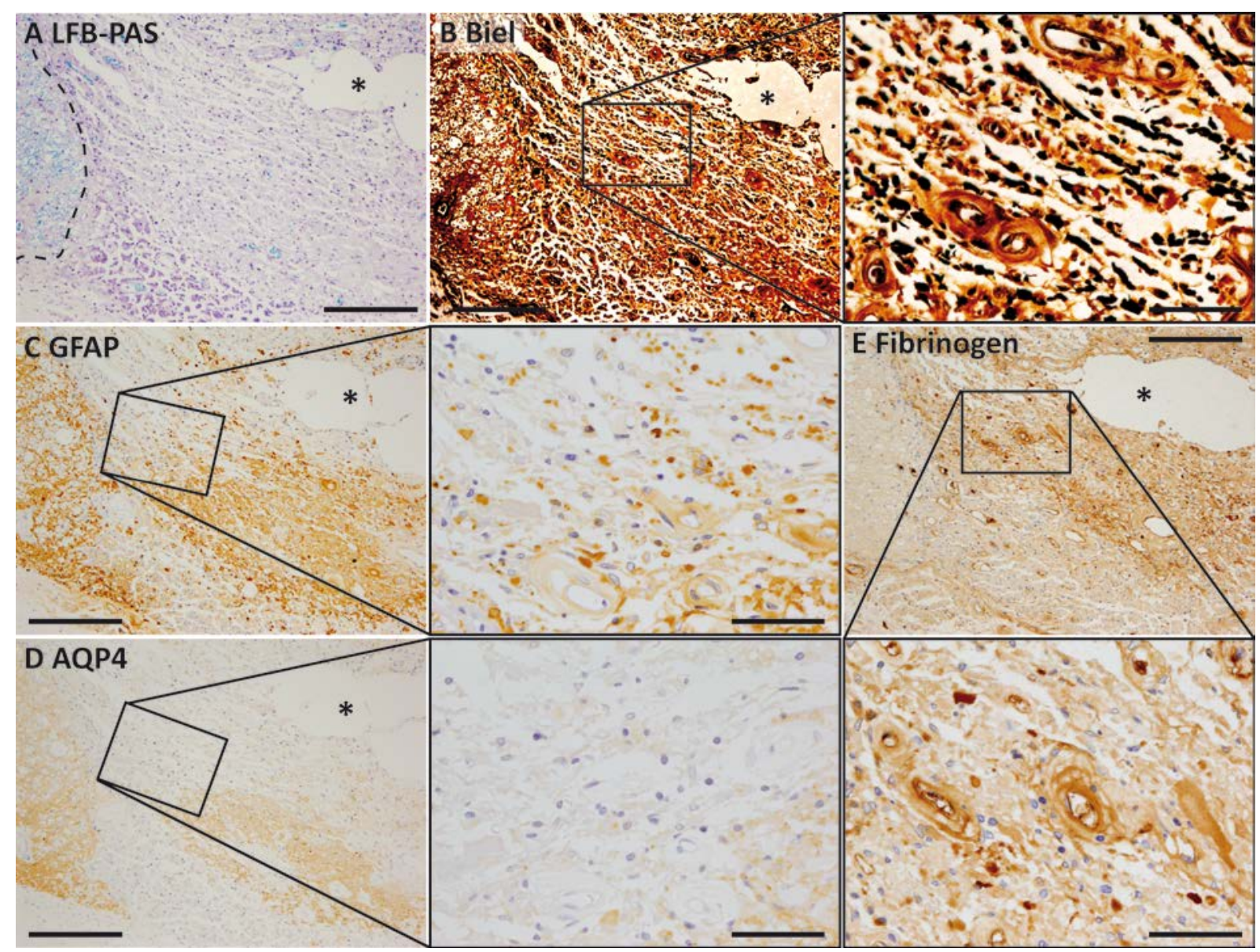

Figure 1: Early NMO lesions are characterized by loss of GFAP and AQP4 immunoreactivity, demyelination, relative axonal preservation and breakdown of the BBB

Representative microphotographs of an early NMO lesion are depicted (autopsy tissue, patient 6). NMO lesions present with demyelination (A, myelin is stained in blue, dotted line indicates lesion border) with relative axonal preservation (B). Loss of astrocytes is apparent in GFAP (C, brown) and AQP4 (D, brown) immunohistochemistry with loss of AQP4 immunoreactivity exceeding the area of GFAP loss. Deposits of fibrinogen indicate loss of BBB integrity (E, brown). Asterisks indicate the same vessel in serial sections. Scale bar $200 \mu \mathrm{m}$, scale bar in inserts $50 \mu \mathrm{m}$

\subsubsection{The blood-brain barrier in NMO}

Although the NMO-Ab present in NMO patients was shown to be pathogenic in animal experiments, it is not known precisely which conditions result in its pathogenicity. In a case report one patient was described to have been seropositive for NMO-Ab more than 10 years before disease onset (Nishiyama et al., 2009). This is supported by observations in vivo where intravenous (i.v.) injection of NMO-Ab in rats did not result in lesion formation (Bradl et al., 2009) which indicates that an intact BBB prevents the passage of the NMO-Ab into the brain parenchyma. Attempts to deliver NMO-Ab to the CNS using juvenile rats in which the BBB was described to be leaky did also not result in lesion formation (Bradl et al., 2009). Similar results were obtained after mechanical brain injury in NMO-Ab seropositive mice (Bradl et al., 2009; Ratelade et al., 2011). This indicates that a second trigger beside the 
impairment of the BBB may be required for the NMO-Ab to become fully pathogenic. Indeed it was demonstrated that the induction of non-specific inflammation and the disruption of the BBB by treatment with complete Freund's adjuvant is sufficient to induce the development of NMO-like lesions in seropositive rats (Kinoshita et al., 2010).

The BBB in NMO patients is severely impaired which is evidenced by the presence of multiple gadolinium enhancing lesions in the CNS on MRI (de Seze et al., 2002; Ito et al., 2009; Kim et al., 2015; Wingerchuk et al., 1999). The disruption of the BBB is associated with edema and infiltration of inflammatory cells. Furthermore, it was demonstrated that the disease severity correlates with the extent of BBB permeability. Investigating the ratio of cerebrospinal fluid (CSF):serum albumin as a measure for BBB permeability Tomizawa and coworkers demonstrated that the breakdown of the BBB in NMO is more severe than in MS and correlates with expanded disability status scale (EDSS) scores during the acute phase of the disease (Tomizawa et al., 2012).

Furthermore it was shown in vitro that incubation of brain endothelial cells (EC) with serum isolated from NMO-patients during the acute phase of the disease increases the transendothelial permeability and decreases the expression of the tight junction (TJ) protein claudin-5. This was significant compared to serum from MS patients or healthy controls (Shimizu et al., 2012; Tasaki et al., 2014). Furthermore, upregulation of cytokines (Chemokine ( $C-X-C$ motif) ligand ( $C X C L) 10$, Chemokine ( $C-C$ motif) ligand $(C C L) 2, C C L 5$, interleukin (IL)-6), the adhesion molecule VCAM-1, the matrix metalloproteinases (MMPs) -2 and -9 and vascular endothelial growth factor (VEGF) by brain EC were observed after treatment with NMO patient serum (Shimizu et al., 2015; Shimizu et al., 2012; Tasaki et al., 2014). However, how serum from NMO patients mediates these effects is so far not known. The authors proposed that humoral factors present in the blood during acute NMO disease phases might play a role, including autoantibodies directed against EC. In vitro work of a second group investigated the effect of the NMO-Ab on the permeability of endothelial monolayers in a co-culture model of the BBB consisting of astrocytes and EC. They demonstrated that astrocytes express AQP4 in a polarized pattern, concentrated on the astrocytic endfeet. Binding of NMO-Ab to AQP4 leads to a depolarized expression and internalization of AQP4 which in turn results in an increase in the permeability of the endothelial/astrocyte barrier (Vincent et al., 2008). In conclusion, the integrity of the BBB in $\mathrm{NMO}$ is severely impaired and the degree of BBB permeability correlates with the EDSS score 
during the acute phase of the disease. However, how the permeability of the BBB is regulated during the disease course is not known.

\subsection{The BBB}

The phenomenon of the BBB was first noted by Paul Ehrlich. He observed that after peritoneal injection of hydrophilic dyes all peripheral organs were stained but not the CNS. He presumed that this difference in staining intensity was due to the different binding affinities of various tissues to different dyes (Ehrlich, 1885). The concept of the BBB as a highly selective permeability barrier was developed only later with the conclusion that "brain capillaries must hold back certain molecules" (Goldmann, 1913; Lewandowski, 1900). Subsequently, this observation was associated with the molecular correlates at the level of EC, the TJ which seal the intercellular gap between adjacent EC and prevent paracellular leakage of solutes (Reese and Karnovsky, 1967). In addition, the BBB controls movements of nutrients, electrolytes, neuroactive agents and neurotransmitters as well as potential neurotoxins from the blood into the brain, and furthermore mediates the efflux of waste products. Thereby the BBB forms a physical, metabolical and transport barrier that is crucial for the maintenance of the unique CNS environment which enables neuronal function (Abbott et al., 2010; Abbott et al., 2006; Wolburg et al., 2008).

\subsubsection{The BBB phenotype of brain endothelial cells}

Brain EC express a variety of transporters and specialized enzyme systems which results in the selective uptake of polar molecules from the blood into the brain. Solute carriers transport polar molecules and supply the brain with nutrients such as glucose (e.g. via glucose transporter 1), amino acids (e.g. via the L-system for large neutral amino acids or the glycine transporter), nucleosides, nucleotides, nucleobases (e.g. via the equilibrative nucleoside transporter 1), and organic ions (e.g. organic cation transporters) (Boado et al., 1999; Borowsky et al., 1993; Gorboulev et al., 1997; Griffiths et al., 1997; Mueckler et al., 1985). Solute carriers not only facilitate the transport of molecules into the brain, but also the transport of waste products back from the brain into the blood. Beside solute carriers, ATP-binding cassette $(A B C)$ transporters play an important role for the BBB phenotype. They restrict the entry of lipophilic molecules into the brain and function as active efflux pumps to remove potentially neurotoxic compounds from the CNS. At the BBB, e.g. P-glycoproteins, multidrug resistance-associated proteins and breast cancer resistance proteins are expressed (Eisenblätter et al., 2003; Juliano and Ling, 1976; Potschka et al., 2003; Zhang et 
al., 2000). A third transport pathway is the transcytosis of macromolecules which can be either receptor- or adsorption mediated (Drin et al., 2003; Pan et al., 2000; Pardridge et al., 1990; Visser et al., 2004; Zlokovic et al., 1990). However, endocytotic vesicles in brain EC are less frequent than in the endothelium of other organs although they might increase during BBB inflammation (Claudio et al., 1989).

The selectivity of the transport into the brain is maintained by the formation of a physical barrier restricting the paracellular diffusion of molecules. Brain EC develop tight interendothelial junctions, i.e. the TJ, which restrict the paracellular movement of even ions like $\mathrm{Na}^{+}$and $\mathrm{Cl}^{-}$. The transendothelial resistance (TEER) is a measure of this ion flux restriction and reaches values of about $1800 \Omega \Omega^{*} \mathrm{~cm}^{2}$ in the brain endothelium of adult rats while values in peripheral capillaries range only from 2-20 $\Omega^{*} \mathrm{~cm}^{2}$ (Butt et al., 1990).

The ultrastructure of TJ at the endothelial junctions in the brain was first described in 1967 using electron microscopy (EM) (Reese and Karnovsky, 1967). Today it is known that the TJ consist of transmembrane linker proteins and cytoplasmic proteins. Three families of transmembrane linker proteins are known: (i) claudins, (ii) TJ-associated MARVEL (MAL and related proteins for vesicle trafficking and membrane link) proteins, including occludin (Furuse et al., 1993), tricellulin (Ikenouchi et al., 2005) and marvelD3 (Steed et al., 2009) and (iii) immunoglobulin superfamily membrane proteins, including junctional adhesion molecules (JAM)-A, JAM-B and JAM-C (Aurrand-Lions et al., 2001) and ESAM (endothelial cell-selective adhesion molecule) (Nasdala et al., 2002). These transmembrane proteins interact via their cytoplasmic domains with intracellular proteins, such as zonula occludens 1 (ZO1), ZO2 and ZO3, which connect the TJ to the actin cytoskeleton and form the backbone of the TJ plaque (Gumbiner et al., 1991; Stevenson et al., 1986). In addition numerous regulatory and signaling molecules are described to be located at the intracellular TJ plaque including small GTPases, ZO-1-associated nucleic acid-binding protein (ZONAB) or protein kinase C subtypes (PKC and PKC $\lambda$ ) (Balda et al., 2003; Citi et al., 2011; Gopalakrishnan et al., 1998; Suzuki et al., 2002; Yamanaka et al., 2001). In addition, the cell-cell contacts in the junctional zone are stabilized by adherens junctions (AJ) (Schulze and Firth, 1993).

\subsubsection{Occludin}

Occludin was the first TJ-specific transmembrane protein to be discovered. It was described to be localized at the TJ in chicken (Furuse et al., 1993) and later also in mammals (AndoAkatsuka et al., 1996). Occludin has a molecular size of about 60-65 kDa and consists of four transmembrane helices, two extracellular loops, a short intracellular $\mathrm{N}$-terminus and a long 
coiled coil containing C-terminal tail. Its overexpression was shown to increase TEER values in vitro (Balda et al., 1996; McCarthy et al., 1996). However, in TJ deficient insect cells or mouse fibroblasts, occludin expression failed to induce de novo formation of intercellular TJ (Furuse et al., 1996; Furuse et al., 1998b). Furthermore, occludin deficient mice show an intact TJ morphology in intestinal epithelial cells, both by immunofluorescence and freeze fracture EM. No difference between wild type (wt) and occludin deficient mice regarding the electrophysiological barrier function of the intestinal epithelium was observed. However, occludin deficient mice display abnormalities in several tissues, like chronic gastritis, calcification of the brain and atrophy of the testis (Saitou et al., 2000). These data indicate that occludin is not the central TJ protein to seal the paracellular cleft. However, a regulatory and accessory function for occludin in TJ formation and physiology is assumed. It was shown that occludin is targeted by several kinases (Chen et al., 2002; Sakakibara et al., 1997; Tsukamoto and Nigam, 1999; Wong, 1997). For example VEGF-induced occludin phosphorylation was demonstrated to result in increased permeability (Harhaj et al., 2006) which was also correlated with occludin degradation in a model of retinopathy (Murakami et al., 2009). Another example is the Src mediated occludin phosphorylation after focal ischemia, also resulting in increased permeability (Kago et al., 2006; Takenaga et al., 2009).

\subsubsection{Claudins}

Claudins constitute the backbone of the TJ strands. To date 27 members of the claudin family have been identified (Mineta et al., 2011). Like occludin, claudins consist of four membrane spanning domains, two extracellular loops and cytosolic $\mathrm{N}$ - and $\mathrm{C}$-terminal domains and have a molecular size of 20-27 kDa (Furuse et al., 1998a; Morita et al., 1999). Claudins are expressed in a tissue-specific manner. They do not only function in the sealing of the paracellular cleft but also form ion-selective pores with a specific pore size and charge selectivity in different tissues. One example is claudin-16 which forms selective pores for the reaborption of $\mathrm{Mg}^{2+}$ and $\mathrm{Ca}^{2+}$ ions in the kidney and is therefore selective for bivalent, but not monovalent cations (Simon et al., 1999).

In the brain, claudin-5 was shown to be the key contributor to TJ formation and BBB integrity. In addition, claudin-3 and -12 are localized at the TJ of the BBB, however with significantly lower expression levels (Daneman et al., 2010a; Liebner et al., 2000b; Nitta, 2003; Ohtsuki et al., 2008; Wolburg et al., 2003). The localization of claudin-1 at the BBB has been controversial as certain Ab show cross-reactivity with claudin-3 (Nitta, 2003; Wolburg et al., 2003). 
Numerous studies have demonstrated claudin- 5 to be important in the sealing of the BBB. Indeed, in vitro studies showed that exogenous expression of claudin-5 enhances barrier properties in rat brain EC (Ohtsuki et al., 2007) while the disruption of claudin-5 increases the paracellular permeability (Luissint et al., 2012). Using knockout mice, claudin-5 was shown to be crucial for the sealing of the BBB to small molecules of $<800 \mathrm{Da}$. Interestingly, TJ appeared normal on the ultrastructural level in claudin-5 deficient mice, indicating that other TJ proteins can partially compensate for the loss of claudin-5. Using immunohistochemistry claudin-12 and ZO1 were demonstrated to be located at the TJ. Claudin-5 deficient mice die within $10 \mathrm{~h}$ after birth although the cause of death is not known (Nitta, 2003).

Claudin-3 was shown to be important for TJ formation at the level of the blood-CFS barrier. Its expression levels are higher in the choroid plexus than in brain capillaries (Kooij et al., 2013; Kratzer et al., 2012). Claudin-3 deficient mice were reported to be viable and fertile and to have no apparent phenotype. However, enhanced blood-CSF permeability was detected using Evans blue as a tracer molecule, which indicates an important role for claudin-3 in the formation of the blood-CSF barrier. Additionally, when challenged, these mice displayed an earlier EAE disease onset and exacerbated disease course compared to wt mice (Kooij et al., 2013).

\subsubsection{Development and maintenance of the BBB}

The development and maintenance of the BBB is dependent on cellular and non-cellular components which were shown to interact with EC and form the so called neurovascular unit (NVU, Figure 2). The NVU comprises EC, astrocytes, pericytes (at the level of capillaries), vascular smooth muscle cells (at the level of arteries), the basal lamina, neurons and microglia (Abbott et al., 2006). Together these cells compose a dynamic structure that is able to respond to peripheral or central cues by the regulation of the permeability of the BBB and the expression of TJ and transporter molecules. During inflammation the NVU can also regulate the expression and presentation of adhesion molecules or chemokines on the endothelium. 


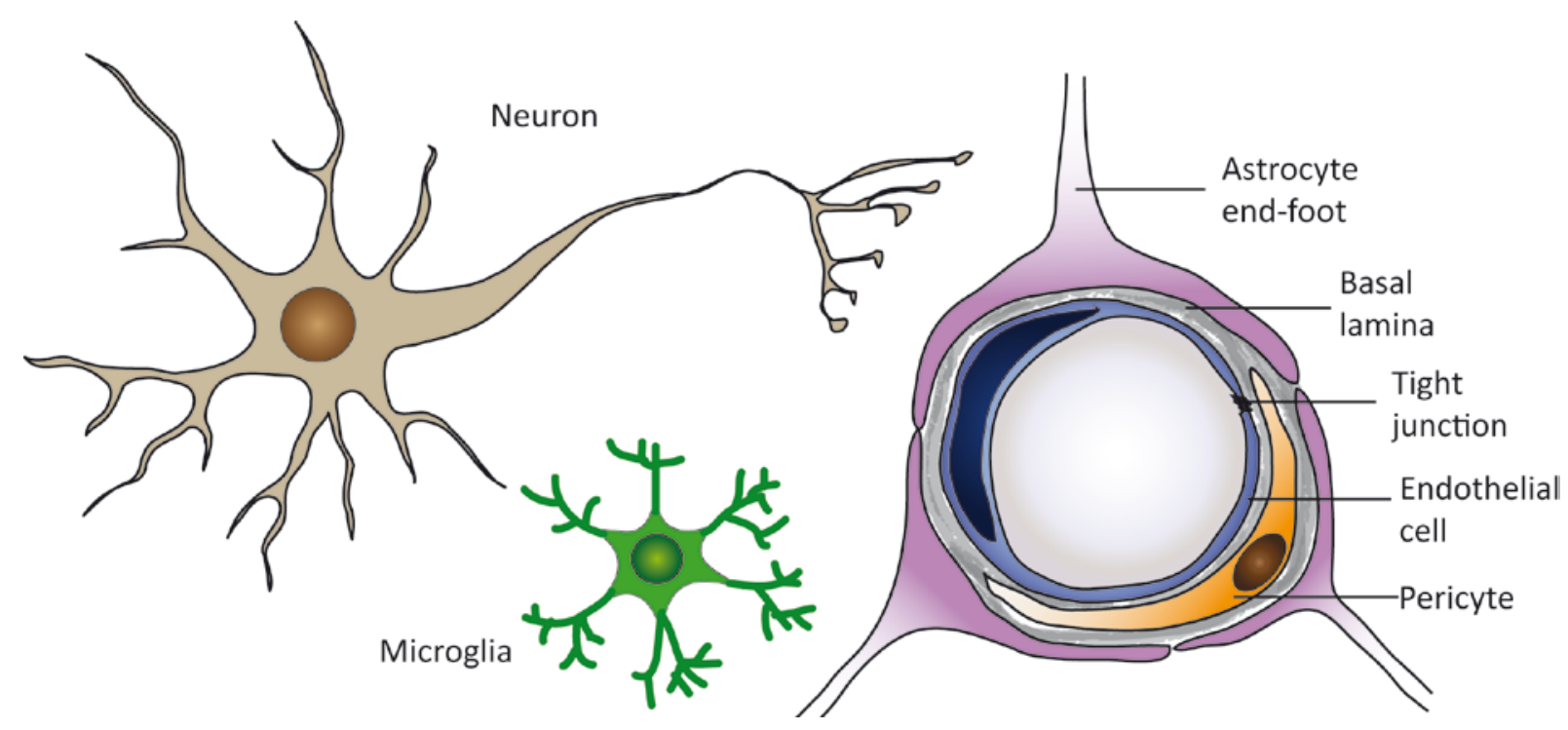

Figure 2: Cellular and structural components of the neurovascular unit

The BBB is composed of brain EC, a surrounding basal lamina, pericytes and astrocytic endfeet. These components are in close contact. Astrocytic and pericytic signaling where shown to be crucial for the development and maintenance of the BBB phenotype of brain EC. In addition, the broader term NVU includes other brain cells such as neurons and microglia which were also shown to directly or indirectly interact with EC.

Furthermore, the interplay between the components of the NVU is essential for the development and maintenance of the BBB. The BBB develops during embryogenesis, and its characteristics are fully developed by birth. During embryonic development mesoderm derived angioblasts were shown to invade the head region where they form the perineural vascular plexus, a vascular network that covers the neural tube. Subsequently, vascular sprouts start to invade the neural tube originating from the perineural vascular plexus and thereby form the CNS vasculature (Bar, 1983; Feeney and Watterson, 1946; Strong, 1964). The induction of BBB defining properties in the vasculature is dependent on the cellular and molecular crosstalk between the sprouts and the neuroectoderm. This was demonstrated in early quail to chicken transplantation studies. When non-vascularized neural tissue from quail embryos was transplanted into the coelomic cavity of chick embryos, abdominal host vessels vascularized the grafted tissue and adopted a BBB phenotype. In contrast, brain vessels vascularizing transplanted mesodermal tissue did not develop BBB characteristics (Stewart and Wiley, 1981).

CNS angiogenesis depends mainly on two signaling molecules: VEGF and Wht both of which are produced by neural progenitor cells. In the brain VEGF is secreted by neural progenitor cells in the subventricular neuroectoderm resulting in the formation of a VEGF concentration gradient that guides sprouting vessels (Carmeliet et al., 1996; Raab et al., 2004). While VEGF 
has a crucial role in the angiogenesis in all tissues, the Wnt/ß-catenin pathway is specific for the angiogenesis in the CNS. Mice deficient of the two Wnt ligands Wnt7a and Wnt7b die around embryonic day 12.5 and present severe hemorrhage and abnormal vessel morphology (Daneman et al., 2009; Stenman et al., 2008). In addition, the Wnt/ß-catenin pathway plays a role in BBB maturation, i.e. TJ formation as ß-catenin depletion in EC resulted in reduced expression of claudin-3 and -5 . This observation was accompanied by an increased permeability to the tracer dye Evans blue (Liebner et al., 2008). Another factor implied in the maturation of the BBB is sonic hedgehog (Shh). Mouse embryos deficient in Shh show a decreased expression of TJ proteins such as occludin and claudin-5 despite having normal numbers of blood vessels. Moreover, selective depletion of the downstream signaling protein smoothened from EC results in lower TJ protein expression and vessel leakage of plasma proteins (Alvarez et al., 2011). Other signaling molecules suggested to play a role in the maturation and differentiation of the BBB are, e.g., transforming growth factor ß (TGF-ß), angiopoietin 1 (Ang-1), angiotensin II (AGT-II) and apolipoprotein E (ApoE) (Dohgu et al., 2005; Lee et al., 2003; Nishitsuji et al., 2011; Wosik et al., 2007b).

Cellular and molecular crosstalk between EC and brain parenchymal cells is important for the development of the BBB. Also in the adult brain, the CNS microenvironment appears to be crucial for the maintenance of the BBB as indicated by numerous in vitro studies. Once brain EC are isolated they lose BBB properties, resulting, e.g., in transcriptional changes and increased permeability (Butt et al., 1990; Demeuse et al., 2002; Lyck et al., 2009; Maxwell et al., 1987). Co-culture of isolated brain EC with either astrocytes, pericytes or neurons or combinations of these cells was shown to partly rescue their BBB phenotype. An increase in TEER values, reduction of permeability, induction of tighter TJ and an enhanced expression and polarization of transporters was observed under co-culturing conditions (Berezowski et al., 2004; Demeuse et al., 2002; Maxwell et al., 1987; Nakagawa et al., 2009; Nakagawa et al., 2007; Perriere et al., 2007; Schiera et al., 2003).

\subsubsection{Pericytes}

Pericytes enwrap the abluminal side of capillaries, small arterioles and venules. They are in close proximity to EC, only separated by the basal lamina (King and Schwyn, 1970; Movat and Fernando, 1964; Murakami et al., 1979). Although pericytes form a rather heterogeneous cell population with no distinct, pericyte-specific marker, platelet-derived growth factor receptor $ß$ (PDGFR-ß) was suggested as a cell specific molecule of brain pericytes (Armulik et al., 2010; Bell et al., 2010; Daneman et al., 2010b). Pericyte recruitment 
takes place early during angiogenesis and is mediated by the release of the PDGFR- $ß$ ligand PDGF-b by nascent sprouts which results in the attraction of PDGFR- $\beta$ expressing pericytes (Bjarnegård et al., 2004; Enge et al., 2002; Hellström et al., 1999). PDGF-b and PDGFR-ß deficient mice lack brain pericytes and are embryonically lethal (Lindahl et al., 1997). Moreover, these mice show an increased vascular permeability and altered junctional architecture (Daneman et al., 2010b; Hellström et al., 2001). Additionally a direct correlation between pericyte coverage of capillaries and BBB permeability to tracer molecules in neonatal mice was observed (Daneman et al., 2010b). This indicates a role of pericytes in the induction of a BBB phenotype in brain EC during embryogenesis. In addition, pericyte signaling has been implied in the maintenance of the BBB during adulthood. Using genetically modified mice with significantly decreased pericyte vessel coverage, an increase in BBB permeability (Armulik et al., 2010; Bell et al., 2010) and age dependent reduction of TJ protein expression (Bell et al., 2010) were shown. However, the molecular pathways by which pericytes participate in the maintenance of the BBB are still unknown.

\subsubsection{Astrocytes}

Astrocytes perform multiple functions in the brain including the uptake and recycling of neurotransmitters, the nutrition of neurons, the regulation of extracellular ion levels, the participation in immune reactions and the maintenance of the BBB. Astrocytic endfeet encircle the abluminal side of CNS vessels and have a close physical association with EC. Therefore, a possible regulatory function of astrocytes in the development and maintenance of the BBB has been assumed. Numerous mechanisms by which astrocytes might regulate the permeability of the BBB have been described. The release of src-suppressed C-kinase substrate (SSeCKS) by astrocytes was shown to increase during BBB maturation which resulted in an enhanced TJ protein expression and a decreased BBB permeability (Lee et al., 2003). Furthermore, astrocytes secrete Shh which binds to hedgehog receptors on EC and thereby increases the expression of occludin and claudin-5 (Alvarez et al., 2011). Another mechanism that has been proposed for the regulation of the BBB is the release of ApoE by astrocytes. Indeed, adult ApoE deficient mice show an increase in albumin permeability at the BBB (Methia et al., 2001) which progresses with age (Hafezi-Moghadam et al., 2007). Furthermore, the renin-angiotensin hormone system has been implied in the regulation of BBB permeability. Angiotensinogen (AGT) is expressed and released by astrocytes and is subsequently converted to the active form AGT-II which binds to its type 1 receptor (AT1) on EC. Activation of AT1 induces phosphorylation of occludin which results in a reduction of the 
BBB permeability in vitro. Conversely, AGT deficient mice show a loss of BBB integrity associated with disorganized occludin strands (Wosik et al., 2007b).

Furthermore, activation of astrocytes during CNS inflammation was shown to alter their expression of secreted molecules, e.g., in MS or its animal model EAE. Here, the increase in factors released by astrocytes was shown to have both beneficial and detrimental effects on the integrity of the BBB and the infiltration of immune cells. Enhanced expression of Shh and retinoic acid resulted in protection of the BBB and endothelial immune quiescence (Alvarez et al., 2011; Argaw et al., 2009; Mizee et al., 2014). In contrast, enhanced expression of VEGF-A by astrocytes disrupted occludin and claudin- 5 strands which was accompanied by an increased BBB permeability. Furthermore, inactivation of astrocytic VEGF-A expression reduced BBB breakdown, infiltration of immune cells and disease severity (Argaw et al., 2012; Argaw et al., 2009). These experimental data were supported by the observation that Shh, retinoic acid and VEGF-A are upregulated in reactive astrocytes in MS lesions (Alvarez et al., 2011; Argaw et al., 2009; Mizee et al., 2014; Proescholdt et al., 2002). Contrarily, the expression of AGT was observed to be reduced in perivascular astrocytes of MS lesions which might be mediated by proinflammatory cytokines. As mentioned above, AGT appears to be important for BBB maintenance and therefore a reduction in AGT expression during inflammation was proposed to contribute to the dysfunction of the BBB in MS patients (Wosik et al., 2007b).

\subsubsection{The BBB during neuroinflammation}

During neuroinflammation two major changes are described at the BBB: (i) increases in BBB permeability to solutes associated with disruption of TJ and (ii) the activation of the endothelium contributing to recruitment and activation of immune cells. Under pathologic conditions immune cells such as lymphocytes, monocytes, macrophages and PMN (including neutrophils, eosinophils and basophils) may be recruited into the CNS. Disruption of the BBB integrity and subsequent increases in vascular permeability and leukocyte extravasation were described during CNS inflammation, e.g., in NMO, MS, cerebral malaria and stroke (Brown et al., 1999; Grossman et al., 1986; O'Riordan et al., 1996; Virapongse et al., 1986). In MS, the infiltration of leukocytes into the CNS is thought to be an early event and associated with increases in BBB permeability which may favor the recruitment of additional leukocytes and thereby trigger and amplify neuroinflammation. 
The recruitment of leukocytes into the CNS is a multistep process and involves leukocyte rolling/tethering, arrest, firm adhesion and crawling at the endothelium and finally diapedesis into the parenchyma. The first step is mediated by a transient contact of leukocytes with the endothelium, the tethering and rolling phase. On the luminal side of the endothelium adhesion molecules of the selectin family (L-, E- and P-selectin) are expressed which are recognized by their respective ligands on leukocytes. Alternatively, tethering/rolling can be mediated by binding of the ligand vascular cell adhesion molecule (VCAM)-1 on EC to a4-integrins on leukocytes (Alon et al., 1995; Bernardes-Silva et al., 2001; Carvalho-Tavares et al., 2000). Subsequently, leukocytes slow down their velocity and may become activated by immobilized chemokines presented on the activated endothelium which are recognized by their G-protein coupled receptors. Intracellular signaling results in conformational changes of $\alpha 4$ - and $\beta 2$-integrins on the leukocyte surface transforming from a low to a high affinity/avidity state (Chigaev et al., 2003; Piccio et al., 2002). Activated integrins in turn bind to their respective ligands on the endothelial surface, e.g. VCAM-1, intercellular adhesion molecule (ICAM)-1 or ICAM-2 mediating arrest, adhesion and finally para- or transcellular diapedesis (Berlin et al., 1995; Gorina et al., 2014; Stanimirovic et al., 1997). Which factors favor which route of transmigration is still controversial. Transmigration into peripheral tissue may occur mainly via the paracellular route. However, when PMN encounter tight endothelial junctions like in the CNS or when ICAM-1 levels are high the transcellular route may be preferred (Abadier et al., 2015; Greenwood et al., 1994; Lossinsky et al., 1989; McMenamin et al., 1992; Raine et al., 1990; von Wedel-Parlow et al., 2011; Wolburg et al., 2005; Yang et al., 2005). The transcellular route furthermore enables leukocytes to cross the endothelium without TJ disruption. Moreover, during diapedesis, a transmigratory cup or dome is formed by the EC surrounding the infiltrating leukocytes which also may minimize permeability during transmigration (Carman and Springer, 2004; Petri et al., 2011; Wolburg et al., 2005).

However, under pathologic conditions the disruption of the BBB can be associated with loss of TJ proteins. This may be mediated, e.g., by the release of cytokines both from infiltrating leukocytes and perivascularly located cells such as astrocytes, pericytes or microglia. Indeed, leukocytes were demonstrated to release cytokines (e.g. tumor necrosis factor (TNF)- $\alpha$, interferon (IFN)- $\psi$, IL-6), enzymes (e.g. MMPs) and reactive oxygen species that may directly or indirectly facilitate BBB disruption. Receptors for TNF- $\alpha$ and INF- $\gamma$ were both reported to be expressed on EC, and alterations of the cellular distribution of TJ and AJ proteins after treatment with TNF- $\alpha$ and INF- $\gamma$ have been shown in the periphery (Blum et al., 1997; 
Kallmann et al., 2002; Ozaki et al., 1999). A direct regulation of TJ by these cytokines at the BBB has been controversial. However, TNF- $\alpha$ and INF- $\gamma$ were shown to increase the expression and secretion of chemokines and adhesion molecules by endothelial cells which may enhance leukocyte infiltration (Lombardi et al., 2009; Subileau et al., 2009; Wosik et al., 2007a). In addition, MMPs released during inflammation may play a role in the disruption of the BBB. MMPs are a family of zinc-dependent endopeptidases whose major functions are tissue remodeling and degradation of extracellular matrix components. They are therefore important during development. In addition, MMPs were shown to be upregulated in various neurological disorders, including MS and NMO (Adair et al., 2004; Alexander et al., 2010; Bernal et al., 2009; Hosokawa et al., 2011; Lindberg et al., 2001; Liuzzi et al., 2000; Lorenzl et al., 2002). Furthermore, mice deficient in both MMP9 and MMP2 are resistant to EAE (Agrawal et al., 2006) while pharmacologic inhibition of MMPs results in an ameliorated EAE course (Gijbels et al., 1994; Hewson et al., 1995; Niimi et al., 2013). Especially MMP9 has been suggested to play a role in the extravasation of leukocytes into the parenchyma in various diseases, such as NMO, MS, stroke or traumatic brain injury (Castellanos et al., 2003; Horstmann et al., 2003; Montaner et al., 2003; Suehiro et al., 2004). In NMO and MS elevated MMP9 levels were measured in the CSF and serum of patients (Alexander et al., 2010; Gijbels et al., 1992; Hosokawa et al., 2011; Mandler et al., 2001) as well as in the serum and CNS of animals with EAE (Clements et al., 1997; Kandagaddala et al., 2012; Kieseier et al., 1998; Nygårdas and Hinkkanen, 2002). Although the exact mechanism of BBB regulation by MMP9 is not known it may directly influence the permeability of the endothelium by the degradation of TJ proteins (Agrawal et al., 2006; Bojarski, 2004; Lischper et al., 2010; Liu et al., 2009; Reijerkerk et al., 2006; Yang et al., 2007).

In addition, leukocytes may directly influence BBB permeability by adhesion mediated signaling. Here, ICAM-1 and ß2-integrin interaction was shown to result in intracellular calcium signaling, actin rearrangement, phosphorylation of AJ proteins, endothelial contraction and increased permeability in vitro (Durieu-Trautmann et al., 1994; EtienneManneville et al., 2000; Gautam et al., 1998; Gautam et al., 2000; Turowski et al., 2008). Furthermore, deletion of the C-terminal ICAM-1 domain in ECs inhibited intracellular signaling and prevented leukocyte transmigration (Greenwood et al., 2003; Lyck et al., 2003). 


\subsection{Aim of this thesis}

The overall aim of this work was to characterize the breakdown of the BBB in a model of $\mathrm{NMO}$ in rats and to investigate the underlying mechanisms. Specifically, we were interested to examine whether there is (i) a relationship between BBB breakdown and astrocyte loss, (ii) a correlation between BBB breakdown and the disruption of TJ and (iii) a possible role of immune cells in the induction of BBB permeability. Therefore, this thesis intends to answer the following questions:

(i) Does the depletion of astrocytes from NMO-like lesions coincide with BBB permeability? Astrocytes, which are the main targets of the humoral immune response in NMO, were shown to play a role in the maintenance of the BBB phenotype. To investigate whether the loss of astrocytes in focal NMO-like lesions correlates with the induction of BBB permeability, time course experiments were performed using serum molecules and exogenous tracers as markers for BBB breakdown. Furthermore, the presence of pericytes in NMO-like lesions was investigated.

(ii) Is vascular leakage associated with a disruption of TJ?

The disruption of TJ strands between adjacent EC was demonstrated to be associated with the loss of BBB integrity to solutes. To evaluate TJ in NMO-like lesions, TJ molecules were investigated on the mRNA and protein level. In addition, the ultrastructural TJ morphology was assessed.

(iii) What is the role of leukocytes for BBB disruption and NMO lesion formation? Infiltrating leukocytes can regulate BBB permeability upon transmigration into the CNS and were shown to participate in the formation of NMO-like lesions by ADCC. Therefore, leukocyte infiltration into NMO-like lesions was investigated. PMN were depleted to investigate their contribution to astrocyte depletion and BBB disruption. Using small molecule inhibitors, the roles of the C5a receptor and neutrophil elastase for PMN recruitment and BBB disruption were assessed in vivo. Furthermore, the influence of proteases released by PMN on the permeability of endothelial monolayers was investigated in vitro. 


\section{$2.1 \quad$ Materials}

\subsubsection{Reagents}

Table 1: Reagents

\begin{tabular}{|c|c|}
\hline Reagents & Source of supply \\
\hline $0.9 \% \mathrm{NaCl}$ solution & B. Braun, Germany \\
\hline Acetic acid & Merck Millipore, Darmstadt, Germany \\
\hline Azure II, powder & Merck Millipore, Darmstadt, Germany \\
\hline BD Calibrite ${ }^{\mathrm{TM}}$ & BD Biosciences, Franklin Lakes, NJ, USA \\
\hline BD FACS Shutdown Solution & BD Biosciences, Franklin Lakes, NJ, USA \\
\hline BD FACSClean $^{\text {TM }}$ & BD Biosciences, Franklin Lakes, NJ, USA \\
\hline BD FACSFlow $^{\mathrm{TM}}$ & BD Biosciences, Franklin Lakes, NJ, USA \\
\hline BD Perm/Wash ${ }^{\text {TM }}$ & BD Biosciences, Franklin Lakes, NJ, USA \\
\hline Chemically defined lipid concentrate & Life Technologies GmbH, Darmstadt, Germany \\
\hline Chloral hydrate & Merck Millipore, Darmstadt, Germany \\
\hline Citric acid & Merck Millipore, Darmstadt, Germany \\
\hline DAB (3,3'-Diaminobenzidine) & Sigma-Aldrich Chemie GmbH, Steinheim, Germany \\
\hline DAPI (4',6-diamidino-2-phenylindole) & Sigma-Aldrich Chemie GmbH, Steinheim, Germany \\
\hline $\begin{array}{l}\text { DDSA (2-Dodecenylsuccinic acid } \\
\text { anhydride) }\end{array}$ & Serva Electrophoresis GmbH, Heidelberg, Germany \\
\hline DePeX & VWR international, Darmstadt, Germany \\
\hline $\begin{array}{l}\text { Di-sodium tetraborate decahydrate } \\
\text { (Borax) }\end{array}$ & Merck Millipore, Darmstadt, Germany \\
\hline $\begin{array}{l}\text { DMEM (High Glucose }(4.5 \mathrm{~g} / \mathrm{l}), \mathrm{w} / \mathrm{L}- \\
\text { Glutamine, sodium pyruvate) }\end{array}$ & Gibco, Life Technologies GmbH, Darmstadt, Germany \\
\hline $\begin{array}{l}\text { DMP-30 (2,4,6 } \\
\text { Tris(dimethylaminomethyl)phenol) }\end{array}$ & Serva Electrophoresis GmbH, Heidelberg, Germany \\
\hline DMSO (dimethyl sulfoxide) & Sigma-Aldrich Chemie GmbH, Steinheim, Germany \\
\hline EBM-2 basal medium & Lonza, Basel, Switzerland \\
\hline Eosin G & Merck Millipore, Darmstadt, Germany \\
\hline Ethanol, absolute & Merck Millipore, Darmstadt, Germany \\
\hline FCS (fetal calf serum) & Sigma-Aldrich Chemie GmbH, Steinheim, Germany \\
\hline Fluorescence mounting medium & Dako Deutschland GmbH, Hamburg, Germany \\
\hline $\begin{array}{l}\text { Forene }^{\circledR} 100 \%(\mathrm{~V} / \mathrm{V}) \text { (Isoflurane; 1-chloro- } \\
\text { 2,2,2-trifluoroethyldifluoromethylether) }\end{array}$ & Abbott GmbH \& Co. KG, Wiesbaden, Germany \\
\hline
\end{tabular}




\begin{tabular}{|c|c|}
\hline Reagents & Source of supply \\
\hline $\begin{array}{l}\text { Giemsa's Azur-Eosin-Methylene blue } \\
\text { solution }\end{array}$ & Merck Millipore, Darmstadt, Germany \\
\hline Glutaraldehyde, $25 \%$, aqueous solution & Merck Millipore, Darmstadt, Germany \\
\hline $\mathrm{H}_{2} \mathrm{O}_{2}$ (hydroxic peroxide), $30 \%$ & Merck Millipore, Darmstadt, Germany \\
\hline $\mathrm{HCl}$ (hydrochloric acid) & Merck Millipore, Darmstadt, Germany \\
\hline $\begin{array}{l}\text { HEPES (4-(2-hydroxyethyl)-1- } \\
\text { piperazineethanesulfonic acid)buffer, } 1 \mathrm{M}\end{array}$ & Sigma-Aldrich Chemie GmbH, Steinheim, Germany \\
\hline Hydrocortisone & Sigma-Aldrich Chemie GmbH, Steinheim, Germany \\
\hline Hydrogen peroxide, $30 \%$ solution & Merck Millipore, Darmstadt, Germany \\
\hline Isopropanol & Merck Millipore, Darmstadt, Germany \\
\hline Ketamine, $10 \%$ & Medistar $^{\circledR}$, Ascheberg, Germany \\
\hline L-Ascorbic Acid & Sigma-Aldrich Chemie GmbH, Steinheim, Germany \\
\hline Mayer's hemalum & Merck Millipore, Darmstadt, Germany \\
\hline Methylene blue & Merck Millipore, Darmstadt, Germany \\
\hline $\begin{array}{l}\text { Monastral blue (Copper (II) } \\
\text { phthalocyaninetetrasulfonic acid } \\
\text { tetrasodium salt) }\end{array}$ & Sigma-Aldrich Chemie GmbH, Steinheim, Germany \\
\hline $\begin{array}{l}\mathrm{NaOH} \text { (sodium hydroxide solution), } \\
1 \mathrm{M}\end{array}$ & Merck Millipore, Darmstadt, Germany \\
\hline Nitric acid, $65 \%$ & Merck Millipore, Darmstadt, Germany \\
\hline Osmium tetraoxide, powder & Carl Roth GmbH + Co. KG, Karlsruhe, Germany \\
\hline Paraffin (Paraplast Plus ${ }^{\circledR}$ ) & Tyco Healthcare, Neustadt, Germany \\
\hline PBS (phosphate buffered saline), 10x & Biochrom AG, Berlin, Germany \\
\hline PBS (phosphate buffered saline), sterile & Sigma-Aldrich Chemie GmbH, Steinheim, Germany \\
\hline $\begin{array}{l}\text { Penicillin-streptomycin (10000 U/ml } \\
\text { penicillin; } 10 \mathrm{mg} / \mathrm{ml} \text { streptomycin) }\end{array}$ & Sigma-Aldrich Chemie GmbH, Steinheim, Germany \\
\hline PFA (paraformaldehyde), powder & Merck Millipore, Darmstadt, Germany \\
\hline PLL (Poly-L-Lysin) & Sigma-Aldrich Chemie GmbH, Steinheim, Germany \\
\hline PMX-53 & Teva Pharmaceutical Industries Ltd, Petha Tikva, Israel \\
\hline $\begin{array}{l}\text { Puromycin dihydrochloride from } \\
\text { streptomyces alboniger }\end{array}$ & Sigma-Aldrich Chemie GmbH, Steinheim, Germany \\
\hline Renlam ${ }^{\circledR} \mathrm{M}-1$ & Serva Electrophoresis GmbH, Heidelberg, Germany \\
\hline Silver nitrate & Carl Roth GmbH + Co. KG, Karlsruhe, Germany \\
\hline Sivelestat sodium salt & R\&D Systems GmbH, Wiesbaden-Nordstadt, Germany \\
\hline Sodium carboxymethyl cellulose & Sigma-Aldrich Chemie GmbH, Steinheim, Germany \\
\hline Sodium thiosulfate pentahydrate & Sigma-Aldrich Chemie GmbH, Steinheim, Germany \\
\hline
\end{tabular}




\begin{tabular}{ll} 
Reagents & Source of supply \\
\hline ß-mercaptoethanol & Sigma-Aldrich Chemie GmbH, Steinheim, Germany \\
\hline Tris (tris(hydroxymethyl)aminomethane) & Carl Roth GmbH + Co. KG, Karlsruhe, Germany \\
\hline Triton $^{\circledast}$ X-100 & MP Biomedicals, LLC, Santa Ana, CA, USA \\
\hline Xylazine solution, $20 \mathrm{mg} / \mathrm{ml}$ & Ecuphar, Oostcamp, Belgium \\
\hline Xylene & $\begin{array}{l}\text { Chemsolute, Th. Geyer GmbH \& Co. KG, Renningen, } \\
\text { Germany }\end{array}$
\end{tabular}

\subsubsection{Solutions, buffers and cell culture media}

Table 2: Solutions

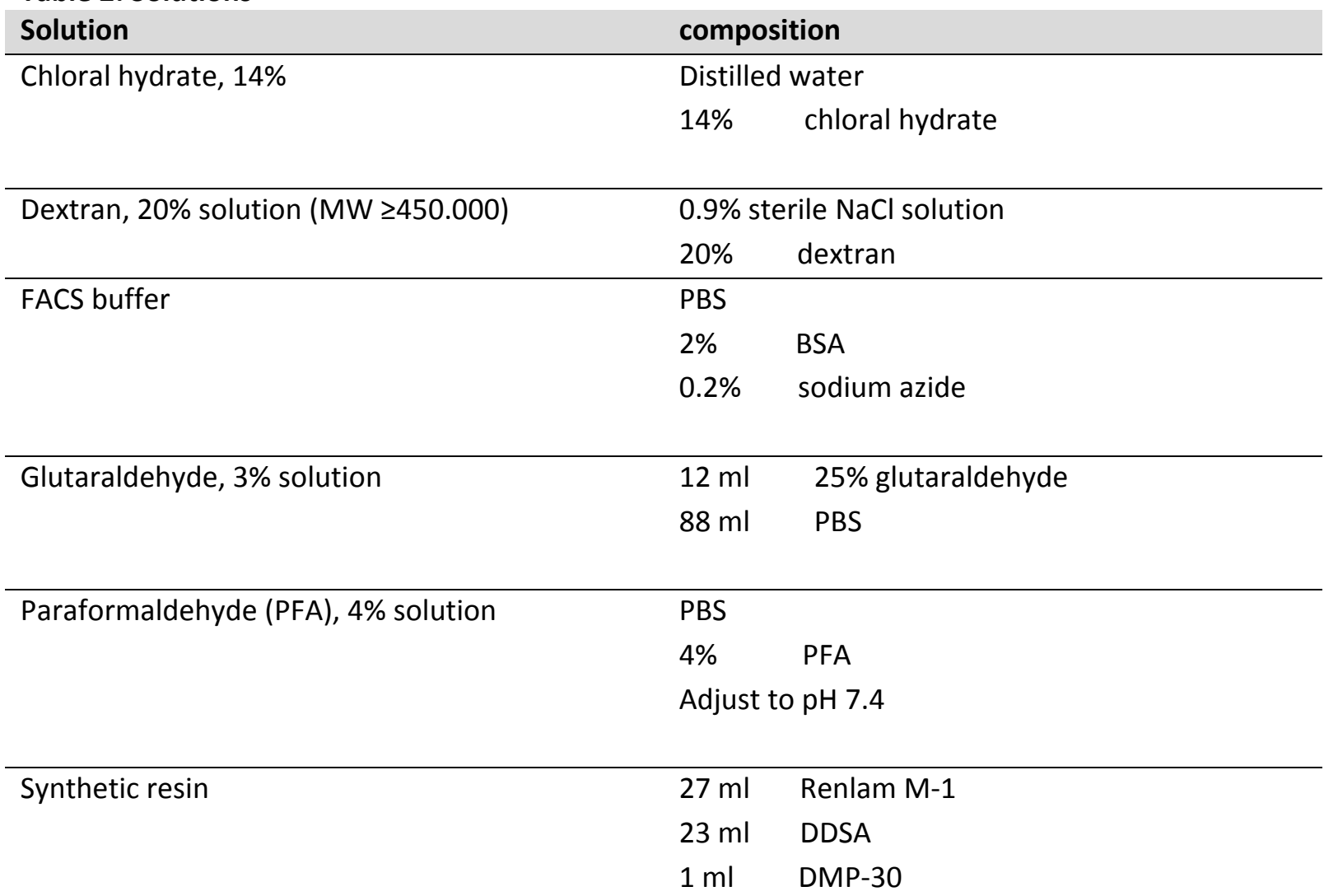

Table 3: Cell culture buffer, media and coating materials

\section{Solution}

0.01\% PLL solution for surface coating (primary astrocytes)

\section{Composition}

PBS, sterile

0.01\% PLL

Incubate over night at $37^{\circ} \mathrm{C}$ 


\begin{tabular}{|c|c|c|}
\hline \multirow{2}{*}{$\begin{array}{l}\text { Solution } \\
\text { Astrocyte culture medium }\end{array}$} & \multicolumn{2}{|c|}{ Composition } \\
\hline & \multicolumn{2}{|l|}{ DMEM } \\
\hline & $10 \%$ & FCS \\
\hline & $100 \mathrm{U} / \mathrm{ml}$ & penicillin \\
\hline & $0.1 \mathrm{mg} / \mathrm{ml}$ & streptomycin \\
\hline \multirow[t]{2}{*}{ RBEC density centrifugation medium } & \multicolumn{2}{|l|}{ DMEM } \\
\hline & $20 \%$ & BSA \\
\hline \multirow[t]{7}{*}{ RBEC digestion buffer } & \multicolumn{2}{|l|}{ DMEM } \\
\hline & $100 \mathrm{U} / \mathrm{ml}$ & penicillin \\
\hline & $0.1 \mathrm{mg} / \mathrm{ml}$ & streptomycin \\
\hline & $10 \mathrm{mM}$ & HEPES \\
\hline & $1 \mathrm{U} / \mathrm{ml}$ & Dispase II \\
\hline & $10 \mathrm{U} / \mathrm{ml}$ & DNase I \\
\hline & $0,1 \mathrm{U} / \mathrm{ml}$ & Collagenase D \\
\hline \multirow[t]{4}{*}{ RBEC dissection buffer } & \multicolumn{2}{|l|}{ DMEM } \\
\hline & $100 \mathrm{U} / \mathrm{ml}$ & penicillin \\
\hline & $0.1 \mathrm{mg} / \mathrm{ml}$ & streptomycin \\
\hline & $10 \mathrm{mM}$ & HEPES \\
\hline \multirow[t]{2}{*}{ RBEC experimental medium } & \multicolumn{2}{|c|}{ RBEC growth medium } \\
\hline & $140 \mu \mathrm{M}$ & hydrocortisone \\
\hline \multirow[t]{8}{*}{ RBEC growth medium } & \multicolumn{2}{|l|}{ EBM-2 } \\
\hline & $5 \%$ & FCS \\
\hline & $1 \%$ & $\begin{array}{l}\text { chemically defined lipid } \\
\text { concentrate }\end{array}$ \\
\hline & $100 \mathrm{U} / \mathrm{ml}$ & penicillin \\
\hline & $0.1 \mathrm{mg} / \mathrm{ml}$ & streptomycin \\
\hline & $5 \mu \mathrm{g} / \mathrm{ml}$ & ascorbic acid \\
\hline & $10 \mathrm{mM}$ & HEPES \\
\hline & $1 \mathrm{ng} / \mathrm{ml}$ & basic fibroblast growth factor \\
\hline \multirow[t]{2}{*}{ RBEC selection medium } & \multicolumn{2}{|c|}{ RBEC growth medium } \\
\hline & $4 \mu \mathrm{g} / \mathrm{ml}$ & puromycin dihydrochloride \\
\hline \multirow[t]{4}{*}{ Surface coating for RBEC } & \multicolumn{2}{|c|}{ Distilled water, sterile } \\
\hline & \multicolumn{2}{|c|}{$50 \mu \mathrm{g} / \mathrm{ml}$ collagen IV } \\
\hline & \multicolumn{2}{|c|}{$50 \mu \mathrm{g} / \mathrm{ml}$ fibronectin } \\
\hline & \multicolumn{2}{|c|}{ Pipette thin layer into insert and air dry } \\
\hline
\end{tabular}

Abbreviations: RBEC: rat brain endothelial cells 
Table 4: Solutions for histochemistry, immunohistochemistry and electron microscopy

\begin{tabular}{|c|c|}
\hline Solution & Composition \\
\hline \multirow[t]{4}{*}{$1 \%$ eosin } & isopropyl alcohol \\
\hline & $\operatorname{eosin} \mathrm{G}$ \\
\hline & Stir, filter \\
\hline & Before use add 0,5\% acetic acid \\
\hline \multirow[t]{2}{*}{$1 \% \mathrm{HCl}$} & $\mathrm{HCl}$ absolute \\
\hline & ethanol \\
\hline \multirow[t]{3}{*}{$10 \mathrm{mM}$ citric acid buffer } & distilled water \\
\hline & Citric acid \\
\hline & Adjust to $\mathrm{pH} 6$ \\
\hline \multirow{2}{*}{$\begin{array}{l}2 \% \text { sodium thiosulfate solution for Bielschowsky } \\
\text { silver impregnation }\end{array}$} & distilled water \\
\hline & sodium thiosulfate pentahydrate \\
\hline \multirow{2}{*}{$\begin{array}{l}20 \% \text { silver nitrate solution for Bielschowsky } \\
\text { silver impregnation }\end{array}$} & distilled water \\
\hline & silver nitrate \\
\hline
\end{tabular}

\begin{tabular}{|c|c|c|}
\hline \multirow{3}{*}{$\begin{array}{l}3,3^{\prime} \text {-diaminobenzidine tetrachloride (DAB) } \\
\text { working solution }\end{array}$} & \multicolumn{2}{|l|}{ PBS } \\
\hline & \multicolumn{2}{|c|}{$0.5 \mathrm{mg} / \mathrm{ml} \quad \mathrm{DAB}$} \\
\hline & \multicolumn{2}{|c|}{$\begin{array}{l}\text { Add } 20 \mu \mathrm{l} 30 \% \text { hydrogen peroxidase per } 50 \mathrm{ml} \\
\text { DAB solution before use }\end{array}$} \\
\hline \multirow[t]{2}{*}{ Blocking buffer for immunohistochemistry } & PBS & \\
\hline & $10 \%$ & FCS \\
\hline \multirow[t]{3}{*}{ Citric acid buffer, $10 \mathrm{mM}$} & $2.1 \mathrm{~g}$ & citric acid \\
\hline & & distilled water \\
\hline & \multicolumn{2}{|c|}{$\mathrm{NaOH}$, adjust to $\mathrm{pH} 6$} \\
\hline \multirow{4}{*}{$\begin{array}{l}\text { Developer stock solution for Bielschowsky silver } \\
\text { impregnation }\end{array}$} & $100 \mathrm{ml}$ & distilled water \\
\hline & $0.5 \mathrm{~g}$ & Citric acid \\
\hline & $20 \mathrm{ml}$ & $37 \%$ formalin \\
\hline & 2 drops & $65 \%$ nitric acid \\
\hline \multirow[t]{3}{*}{ Richardson's stain } & $10 \mathrm{ml}$ & $1 \%$ Azure II \\
\hline & $5 \mathrm{ml}$ & $2 \%$ methylene blue \\
\hline & $5 \mathrm{ml}$ & $1 \%$ borax \\
\hline
\end{tabular}




\subsubsection{Antibodies, enzymes and proteins}

Table 5: Primary antibodies for immunohistochemical staining

\begin{tabular}{|c|c|c|c|c|c|c|}
\hline Antigen & Marker for & $\begin{array}{l}\text { Species/ } \\
\text { clone }\end{array}$ & $\mathrm{P} / \mathrm{C}$ & $\begin{array}{l}\text { Antigen } \\
\text { retrieval/ } \\
\text { fixation }\end{array}$ & $\begin{array}{l}\text { Working } \\
\text { dilution }\end{array}$ & Source of supply \\
\hline AQP4 & $\begin{array}{l}\text { aquaporin4/ } \\
\text { water channel, } \\
\text { expressed in the } \\
\text { brain on } \\
\text { astrocytic endfeet }\end{array}$ & rabbit & $P$ & - & $1: 100$ & $\begin{array}{l}\text { Merck Millipore, } \\
\text { Darmstadt, } \\
\text { Germany }\end{array}$ \\
\hline CD3 & $\begin{array}{l}\text { cluster of } \\
\text { differentiation 3/ } \\
\text { T-cell receptor }\end{array}$ & $\begin{array}{l}\text { rat/ } \\
\text { CD3-12 }\end{array}$ & $P$ & $\begin{array}{l}\text { microwave, } \\
\text { citrate } \\
\text { buffer }\end{array}$ & $1: 200$ & $\begin{array}{l}\text { AbD Serotec, } \\
\text { Puchheim, } \\
\text { Germany }\end{array}$ \\
\hline CD68 (ED1) & $\begin{array}{l}\text { cluster of } \\
\text { differentiation } \\
68 / \text { macrophages } \\
\text { and activated } \\
\text { microglia in rat }\end{array}$ & $\begin{array}{l}\text { mouse/ } \\
\text { ED1 }\end{array}$ & $P$ & $\begin{array}{l}\text { Proteinase } \\
\mathrm{K}\end{array}$ & $1: 500$ & $\begin{array}{l}\text { AbD Serotec, } \\
\text { Puchheim, } \\
\text { Germany }\end{array}$ \\
\hline $\begin{array}{l}\text { CD68 } \\
\text { (KiM1P) }\end{array}$ & $\begin{array}{l}\text { cluster of } \\
\text { differentiation } \\
68 / \text { macrophages } \\
\text { and activated } \\
\text { microglia in } \\
\text { humans }\end{array}$ & mouse & $\mathrm{P}$ & $\begin{array}{l}\text { microwave, } \\
\text { citrate } \\
\text { buffer }\end{array}$ & $1: 5000$ & private \\
\hline Claudin-3 & $\begin{array}{l}\text { Transmembrane } \\
\text { TJ-molecule }\end{array}$ & rabbit & $\mathrm{C}$ & ethanol & $1: 100$ & $\begin{array}{l}\text { Acris Antibodies, } \\
\text { Inc., San Diego, } \\
\text { CA,USA }\end{array}$ \\
\hline Claudin-5 & $\begin{array}{l}\text { Transmembrane } \\
\text { TJ-molecule }\end{array}$ & rabbit & $\mathrm{C}$ & ethanol & $1: 250$ & $\begin{array}{l}\text { Acris Antibodies, } \\
\text { Inc., San Diego, } \\
\text { CA,USA }\end{array}$ \\
\hline Fibrinogen & $\begin{array}{l}\text { blood } \\
\text { glycoprotein, } \\
\text { used as marker } \\
\text { for BBB } \\
\text { breakdown }\end{array}$ & rabbit & $P$ & $\begin{array}{l}\text { proteinase } \\
\mathrm{K}\end{array}$ & $1: 300$ & $\begin{array}{l}\text { Dako Deutschland } \\
\text { GmbH, Hamburg, } \\
\text { Germany }\end{array}$ \\
\hline $\begin{array}{l}\text { FITC, HRP } \\
\text { conjugated }\end{array}$ & $\begin{array}{l}\text { fluorescein } \\
\text { isothiocyanate }\end{array}$ & rabbit & $\mathrm{P}$ & $\begin{array}{l}\text { microwave, } \\
\text { citrate } \\
\text { buffer }\end{array}$ & $1: 50$ & $\begin{array}{l}\text { Dako Deutschland } \\
\text { GmbH, Hamburg, } \\
\text { Germany }\end{array}$ \\
\hline GFAP & $\begin{array}{l}\text { glial fibrillary } \\
\text { acidic protein, } \\
\text { astrocytic } \\
\text { intermediary } \\
\text { filament }\end{array}$ & mouse & $P+C$ & $\begin{array}{l}\text { microwave, } \\
\text { citrate } \\
\text { buffer }\end{array}$ & $1: 300$ & $\begin{array}{l}\text { Synaptic Systems } \\
\text { GmbH, Göttingen, } \\
\text { Germany }\end{array}$ \\
\hline GFAP & $\begin{array}{l}\text { glial fibrillary } \\
\text { acidic protein, } \\
\text { astrocytic } \\
\text { intermediary } \\
\text { filament }\end{array}$ & rabbit & $\mathrm{P}$ & - & $1: 1000$ & $\begin{array}{l}\text { Dako Deutschland } \\
\text { GmbH, Hamburg, } \\
\text { Germany }\end{array}$ \\
\hline
\end{tabular}




\begin{tabular}{|c|c|c|c|c|c|c|}
\hline Antigen & Marker for & $\begin{array}{l}\text { Species/ } \\
\text { clone }\end{array}$ & $\mathrm{P} / \mathrm{C}$ & $\begin{array}{l}\text { Antigen } \\
\text { retrieval/ } \\
\text { fixation }\end{array}$ & $\begin{array}{l}\text { Working } \\
\text { dilution }\end{array}$ & Source of supply \\
\hline GFAP-Cy3 & $\begin{array}{l}\text { glial fibrillary } \\
\text { acidic protein, } \\
\text { astrocytic } \\
\text { intermediary } \\
\text { filament, used for } \\
\text { LCM }\end{array}$ & $\begin{array}{l}\text { mouse/ } \\
\text { G-A-5 }\end{array}$ & $\mathrm{C}$ & - & $1: 80$ & $\begin{array}{l}\text { Abcam, } \\
\text { Cambridge, } \\
\text { England }\end{array}$ \\
\hline LAM 1 & $\begin{array}{l}\text { laminin B2 chain; } \\
\text { component of } \\
\text { basement } \\
\text { membrane }\end{array}$ & $\begin{array}{l}\text { mouse/ } \\
2 \mathrm{E} 8\end{array}$ & $\mathrm{C}$ & ethanol & $1: 100$ & $\begin{array}{l}\text { Merck Millipore, } \\
\text { Darmstadt, } \\
\text { Germany }\end{array}$ \\
\hline MMP9 & $\begin{array}{l}\text { matrix } \\
\text { metalloproteinas } \\
\text { e 9; }\end{array}$ & rabbit & $P$ & $\begin{array}{l}\text { microwave, } \\
\text { citrate } \\
\text { buffer }\end{array}$ & $1: 50$ & $\begin{array}{l}\text { Paesel + Lorei } \\
\text { GmbH \& Co., } \\
\text { Duisburg, Germany }\end{array}$ \\
\hline MRP14 & $\begin{array}{l}\text { myeloid-related } \\
\text { protein-14, } \\
\text { neutrophils and } \\
\text { monocytes }\end{array}$ & $\begin{array}{l}\text { mouse/ } \\
\text { BM } \\
40263\end{array}$ & $P$ & $\begin{array}{l}\text { Proteinase } \\
\mathrm{K}\end{array}$ & $1: 500$ & $\begin{array}{l}\text { Acris Antibodies, } \\
\text { Inc., San Diego, } \\
\text { CA,USA }\end{array}$ \\
\hline Occludin & $\begin{array}{l}\text { Transmembrane } \\
\text { TJ-molecule }\end{array}$ & rabbit & $\mathrm{C}$ & ethanol & $1: 50$ & $\begin{array}{l}\text { Life Technologies } \\
\text { GmbH, Darmstadt, } \\
\text { Germany }\end{array}$ \\
\hline PDGFRß & $\begin{array}{l}\text { platelet-derived } \\
\text { growth factor } \\
\text { receptor on } \\
\text { pericytes }\end{array}$ & $\begin{array}{l}\text { rabbit/ } \\
28 \mathrm{E} 1\end{array}$ & $\mathrm{C}$ & ethanol & $1: 50$ & $\begin{array}{l}\text { Cell Signaling } \\
\text { Technology, Inc. } \\
\text { Danvers, MA, USA }\end{array}$ \\
\hline $\begin{array}{l}\text { rat IgG, } \\
\text { biotinylated }\end{array}$ & rat IgG & rat & $P$ & $\begin{array}{l}\text { Proteinase } \\
\mathrm{K}\end{array}$ & $1: 200$ & $\begin{array}{l}\text { GE Healthcare } \\
\text { Europe GmbH, } \\
\text { Freiburg, Germany }\end{array}$ \\
\hline Texas Red & Texas Red & Rabbit & $P$ & $\begin{array}{l}\text { microwave, } \\
\text { citrate } \\
\text { buffer }\end{array}$ & $1: 200$ & $\begin{array}{l}\text { Life Technologies } \\
\text { GmbH, Darmstadt, } \\
\text { Germany }\end{array}$ \\
\hline vWF & $\begin{array}{l}\text { von Willebrand } \\
\text { Factor; specific } \\
\text { for EC }\end{array}$ & rabbit & $\mathrm{C}$ & ethanol & $1: 70$ & $\begin{array}{l}\text { Abcam, } \\
\text { Cambridge, } \\
\text { England }\end{array}$ \\
\hline
\end{tabular}

Abbreviations: $\mathbf{P}=$ used on paraffin embedded tissue; $\mathbf{C}=$ used on cryopreserved tissue and/or for immunocytochemistry

Table 6: Secondary antibodies for immunohistochemical staining

\begin{tabular}{lll} 
Secondary antibody & Working dilution & Manufacturer \\
\hline anti-mouse IgG, biotinylated & $1: 200$ & $\begin{array}{l}\text { GE Healthcare Europe GmbH, Freiburg, } \\
\text { Germany }\end{array}$ \\
\hline anti-rabbit IgG, biotinylated & $1: 200$ & $\begin{array}{l}\text { GE Healthcare Europe GmbH, Freiburg, } \\
\text { Germany }\end{array}$ \\
\hline anti-rat IgG, biotinylated & $1: 200$ & $\begin{array}{l}\text { GE Healthcare Europe GmbH, Freiburg, } \\
\text { Germany }\end{array}$
\end{tabular}




\begin{tabular}{lll} 
Secondary antibody & Working dilution & Manufacturer \\
\hline $\begin{array}{l}\text { Cy } \\
\text { mouse }(\mathrm{H}+\mathrm{L})\end{array}$ & $1: 200$ & $\begin{array}{l}\text { Jackson ImmunoResearch Laboratories, } \\
\text { Inc., West Grove, PA, USA }\end{array}$ \\
\hline $\begin{array}{l}\mathrm{C} \mathrm{C}^{\mathrm{TM} 3} \text {-conjugated AffiPure goat Anti- } \\
\text { rabbit }(\mathrm{H}+\mathrm{L})\end{array}$ & $1: 200$ & $\begin{array}{l}\text { Jackson ImmunoResearch Laboratories, } \\
\text { Inc., West Grove, PA, USA }\end{array}$
\end{tabular}

Table 7: Monoclonal antibodies for flow cytometry

\begin{tabular}{lllll} 
Specificity & Fluorochrome & Clone & Dilution & Source of supply \\
\hline CD11b & Alexa Fluor 647 & Ox-42 & $1: 100$ & AbD Serotec, Oxford, UK \\
\hline CD4 & PE-Cy5 & OX-35 & 1.100 & $\begin{array}{l}\text { BD Biosciences, Franklin Lakes, } \\
\text { NJ, USA }\end{array}$ \\
\hline CD45RA & PE & OX-33 & $1: 100$ & BioLegend, San Diego, CA, USA \\
\hline CD8a & PerCP & OX-8 & $1: 100$ & BioLegend, San Diego, CA, USA \\
\hline granulocytes & PE & RP-1 & $1: 100$ & $\begin{array}{l}\text { BD Biosciences, Franklin Lakes, } \\
\text { NJ, USA }\end{array}$ \\
\hline TCR $\alpha /$ / & Alexa Fluor 647 & R73 & $1: 100$ & BioLegend, San Diego, CA, USA
\end{tabular}

Abbreviations: $\mathbf{C D}=$ cluster of differentiation; $\mathbf{C y 5}=$ cyanine 5; $\mathbf{P E}=$ phycoerythrin; $\mathbf{P e r C P}=$ peridinin chlorophyll protein; $\mathbf{T C R}=\mathrm{T}$ cell receptor

Table 8: Recombinant human antibodies for induction of focal NMO lesions in vivo

\begin{tabular}{llll} 
Specificity & Clone & Application & Source of supply \\
\hline AQP4 & rAb-53 & $\begin{array}{l}\text { Induction of focal } \\
\text { NMO lesions }\end{array}$ & $\begin{array}{l}\text { J. Bennett, Department of Neurology and } \\
\text { Ophthalmology, University of Colorado, } \\
\text { Denver, USA }\end{array}$ \\
\hline $\begin{array}{l}\text { Measles virus } \\
\text { nucleocapsid } \\
\text { protein }\end{array}$ & rAb-2B4 & $\begin{array}{l}\text { Isotype control } \\
\text { antibody }\end{array}$ & $\begin{array}{l}\text { J. Bennett, Department of Neurology and } \\
\text { Ophthalmology, University of Colorado, } \\
\text { Denver, USA }\end{array}$
\end{tabular}

Table 9: Sera for PMN-depletion in vivo

\begin{tabular}{lll} 
Serum & Application & Source of supply \\
\hline $\begin{array}{l}\text { anti-PMN antiserum } \\
\text { (AIA51140) }\end{array}$ & Depletion of PMN & $\begin{array}{l}\text { Accurate Chemical and Scientific } \\
\text { Corporation, Westbury, NY, USA }\end{array}$ \\
\hline $\begin{array}{l}\text { normal rabbit serum } \\
\text { (AIS403) }\end{array}$ & Control serum & $\begin{array}{l}\text { Accurate Chemical and Scientific } \\
\text { Corporation, Westbury, NY, USA }\end{array}$
\end{tabular}

Table 10: Proteins and enzymes

\begin{tabular}{ll} 
Proteins/ enzymes & Source of supply \\
\hline Albumin-fluorescein isothiocyanate conjugate & $\begin{array}{l}\text { Sigma-Aldrich Chemie GmbH, Steinheim, } \\
\text { Germany }\end{array}$ \\
\hline BSA (bovine serum albumin) & $\begin{array}{l}\text { SERVA Electrophoresis GmbH, Heidelberg, } \\
\text { Germany }\end{array}$
\end{tabular}




\begin{tabular}{|c|c|}
\hline Proteins/ enzymes & Source of supply \\
\hline Cathepsin G from human leukocytes & $\begin{array}{l}\text { Sigma-Aldrich Chemie GmbH, Steinheim, } \\
\text { Germany }\end{array}$ \\
\hline $\begin{array}{l}\text { Collagen from human placenta Bornstein and } \\
\text { Traub Type IV }\end{array}$ & $\begin{array}{l}\text { Sigma-Aldrich Chemie GmbH, Steinheim, } \\
\text { Germany }\end{array}$ \\
\hline Collagenase $\mathrm{D}$ & $\begin{array}{l}\text { Roche Diagnostics Deutschland GmbH, } \\
\text { Mannheim, Germany }\end{array}$ \\
\hline Complement sera human, lyophilized powder & $\begin{array}{l}\text { Sigma-Aldrich Chemie GmbH, Steinheim, } \\
\text { Germany }\end{array}$ \\
\hline Dispase II (neutral protease, grade II) & $\begin{array}{l}\text { Roche Diagnostics Deutschland GmbH, } \\
\text { Mannheim, Germany }\end{array}$ \\
\hline DNase I & $\begin{array}{l}\text { Roche Diagnostics Deutschland GmbH, } \\
\text { Mannheim, Germany }\end{array}$ \\
\hline Elastase, human neutrophil & Merck Millipore, Darmstadt, Germany \\
\hline ExtrAvidin ${ }^{\circledR}$-Peroxidase & $\begin{array}{l}\text { Sigma-Aldrich Chemie GmbH, Steinheim, } \\
\text { Germany }\end{array}$ \\
\hline Fibrinonectin from bovine plasma & $\begin{array}{l}\text { Sigma-Aldrich Chemie GmbH, Steinheim, } \\
\text { Germany }\end{array}$ \\
\hline $\begin{array}{l}\text { Fibroblast Growth Factor-Basic, human, } \\
\text { recombinant expressed in E. coli }\end{array}$ & $\begin{array}{l}\text { Sigma-Aldrich Chemie GmbH, Steinheim, } \\
\text { Germany }\end{array}$ \\
\hline MMP9, active, human, recombinant & Merck Millipore, Darmstadt, Germany \\
\hline Proteinase $\mathrm{K}$ & $\begin{array}{l}\text { Sigma-Aldrich Chemie GmbH, Steinheim, } \\
\text { Germany }\end{array}$ \\
\hline Texas $\operatorname{Red}^{\circledR}$ cadaverine & Life Technologies GmbH, Darmstadt, Germany \\
\hline Trypsin-EDTA (0.25\% and 0.05\%) & $\begin{array}{l}\text { Gibco, Life Technologies GmbH, Darmstadt, } \\
\text { Germany }\end{array}$ \\
\hline
\end{tabular}

\subsubsection{Applied kits}

Table 11: Applied kits

Kit

CytoTox-ONE ${ }^{\text {TM }}$ Homogeneous Membrane Integrity Assay

High-Capacity cDNA Reverse Transcription Kit with RNase Inhibitor

\begin{tabular}{ll}
\hline $\begin{array}{l}\text { Naphthol AS-D Chloroacetate (Specific Esterase) } \\
\text { Kit }\end{array}$ & $\begin{array}{l}\text { Sigma-Aldrich Chemie GmbH, Steinheim, } \\
\text { Germany }\end{array}$ \\
\hline qPCR Core Kit & Eurogentec Deutschland GmbH, Köln, Germany \\
\hline RNeasy Micro Kit & Qiagen GmbH, Hilden, Germany
\end{tabular}

\section{Source of supply}

Promega, Mannheim, Germany

Life Technologies GmbH, Darmstadt, Germany 


\subsubsection{Primers for qPCR}

Table 12: TaqMan $^{\circledR}$ qPCR primers

\begin{tabular}{llll} 
Primer number & RNA specificity & Reference sequence & Source of supply \\
\hline Rn00581751_s1 & claudin-3 & NM_031700.2 & $\begin{array}{l}\text { Technologies GmbH, Darmstadt, } \\
\text { Germany }\end{array}$ \\
\hline Rn01753146_s1 & claudin-5 & NM_031701.2 & $\begin{array}{l}\text { Technologies GmbH, Darmstadt, } \\
\text { Germany }\end{array}$ \\
\hline Rn01775763_g1 & gapdh & NM_017008.4 & $\begin{array}{l}\text { Technologies GmbH, Darmstadt, } \\
\text { Germany }\end{array}$ \\
\hline Rn00580064_m1 & occludin & NM_031329.2 & $\begin{array}{l}\text { Technologies GmbH, Darmstadt, } \\
\text { Germany }\end{array}$
\end{tabular}

\subsubsection{Consumables}

Table 13: Consumables

Consumable

Blaubrand $^{\circledR}$ intraMARK micropipettes, $5 \mu \mathrm{l}$

Bottle top filter, $0.2 \mu \mathrm{m}$

Cell culture dish, $60 \times 15 \mathrm{~mm}$

Cell culture flask, $75 \mathrm{~cm}^{2}$

Cell culture plate, flat bottom (12 well, 96 well)

Falcon 12 well permeable support companion plate

Falcon permeable support for 12 well plate with Corning, Corning, NY, USA $0.4 \mu \mathrm{m}$ transparent PET membrane

\begin{tabular}{ll}
\hline MMI IsolationCaps, diffuse, $0.5 \mathrm{ml}$ & $\begin{array}{l}\text { Molecular Machines \& Industries GmbH, Eching, } \\
\text { Germany }\end{array}$ \\
\hline MMI MembraneSlides, RNAse free & $\begin{array}{l}\text { Molecular Machines \& Industries GmbH, Eching, } \\
\text { Germany }\end{array}$ \\
\hline Needles & BD Biosciences, Franklin Lakes, NJ, USA \\
\hline Syringes & BD Biosciences, Franklin Lakes, NJ, USA \\
\hline Tubes $(50 \mathrm{ml}, 15 \mathrm{ml}, 2 \mathrm{ml}, 1.5 \mathrm{ml}, 0.5 \mathrm{ml}, 0.2 \mathrm{ml})$ & Sarstedt, Nuembrecht, Germany
\end{tabular}

\subsubsection{Technical devices}

Table 14: Technical devices

Device

BD FACSCalibur ${ }^{\text {TM }}$

BX51 Olympus light microscope equipped with DP71 digital and XM10 monochrome camera

Cellstar incubator

Centrifuge $5415 \mathrm{R}$

Centrifuge $5810 \mathrm{R}$

Cryotome CM3050

\section{Source of supply}

Brand $\mathrm{GmbH}$, Wertheim, Germany

Sarstedt, Nuembrecht, Germany

Greiner bio-one, Kremsmuenster, Austria

Greiner bio-one, Kremsmuenster, Austria

Greiner bio-one, Kremsmuenster, Austria

Corning, Corning, NY, USA

Molecular Machines \& Industries $\mathrm{GmbH}$, Eching

Germany

Sarstedt, Nuembrecht, Germany

\section{Source of supply}

BD Biosciences, Franklin Lakes, NJ, USA

Olympus, Hamburg, Germany

Nunc GmbH \& Co. KG, Wiesbaden, Germany

Eppendorf, Hamburg, Germany

Eppendorf, Hamburg, Germany

Leica, Wetzlar, Germany 


\begin{tabular}{ll} 
Device & Source of supply \\
\hline EM10B electron microscope & $\begin{array}{l}\text { Carl Zeiss Microscopy GmbH, Oberkochen, } \\
\text { Germany }\end{array}$ \\
\hline EVOM $^{2}$ Epithelial Voltohmmeter, STX2 electrode & $\begin{array}{l}\text { World Precision Instruments Germany GmbH, } \\
\text { Berlin, Germany }\end{array}$ \\
\hline iCycler iQ ${ }^{\text {TM5 }}$ & Bio-Rad Laboratories GmbH, München, Germany \\
\hline Leica Ultracut UCT & Leica, Wetzlar, German \\
\hline Microtome & Leica, Wetzlar, Germany \\
\hline Microwave & Bosch, Gerling-Schillerhohe, Germany \\
\hline NanoDrop ND-1000 & Peqlab, VWR International GmbH, Erlangen, \\
\hline Stereotactic device & Germany \\
\hline T3 Thermocycler & Stoelting Co, Wood Dale, IL, USA \\
\hline Tecan Safire plate reader & Biometra, Germany \\
\hline TP 1020 (tissue processor) & Tecan Group Ltd., Männedorf, Switzerland \\
\hline
\end{tabular}

\subsubsection{Software}

Table 15: Software

\begin{tabular}{lll} 
Software & Application & Source of supply \\
\hline ImageJ 1.47d & Measurements of lesion size & National Institutes of Health, \\
& and tracer extravasation & Bethesda, Maryland, USA \\
\hline GraphPad Prism 5.01 & Statistical analysis & $\begin{array}{l}\text { GraphPad software Inc., La } \\
\text { Jolla, CA, USA }\end{array}$
\end{tabular}

\subsection{Human neuromyelitis optica (NMO) tissue}

Biopsy or autopsy central nervous system (CNS) tissue from seven anti-AQP4 seropositive NMO or NMO spectrum disorder patients was analyzed to investigate the type and extent immune cell infiltration. In 5/7 patients, brain or spinal cord biopsies were taken for diagnostic reasons to exclude lymphoma, astrocytoma or infection. Additionally, autopsy material from two patients was included in this study. Astrocyte-depleting and demyelinating lesions compatible with NMO or NMO spectrum disorder was confirmed by immunohistochemistry in these patients. Loss of AQP4 and GFAP positive cells was observed in macrophage-rich areas. Inflammatory areas also presented with demyelination and relative axonal preservation and were therefore consistent with early NMO lesions. Immune cell infiltration was investigated in HE (PMN infiltration), MRP14 (PMN/early monocytes), KiM1P (CD68, macrophages/activated microglia) and CD3 (T cells) immunohistochemistry. The studies on human tissue were approved by the local ethics committee (14/5/03). 
Table 16: Clinical data of patients with anti-AQP4 seropositive NMO or NMO spectrum disorder

\begin{tabular}{lllllll}
$\begin{array}{l}\text { Patient } \\
\text { no. }\end{array}$ & B/A & Age/sex & $\begin{array}{l}\text { Disease duration } \\
\text { (years) }\end{array}$ & $\begin{array}{l}\text { disease } \\
\text { course }\end{array}$ & CNS involvement & lesions studied \\
\hline 1 & B & $37 / \mathrm{f}$ & 9 & RR & $\begin{array}{l}\text { Brain, spinal } \\
\text { cord, optic nerve }\end{array}$ & Occipital brain lesion \\
& & & & & &
\end{tabular}

\begin{tabular}{llllll}
\hline 2 & B & $31 / \mathrm{f}$ & 5 & $\mathrm{RR}$ & $\begin{array}{l}\text { Brain, spinal } \\
\text { cord, optic nerve }\end{array}$
\end{tabular}

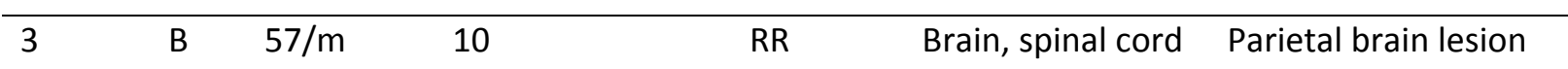

\begin{tabular}{lllllll}
\hline 4 & B & $67 / f$ & $<1$ & ns & Brain & Parietal brain lesion \\
\hline 5 & B & $45 / \mathrm{f}$ & 2 & RR & Spinal cord & spinal lesion \\
\hline 6 & A & $77 / f$ & 24 & RR & $\begin{array}{l}\text { Brain, spinal } \\
\text { cord, optic nerve }\end{array}$ & spinal lesion
\end{tabular}

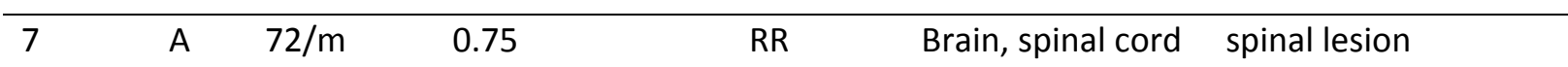

Abbreviations: $\mathbf{A}=$ autopsy, $\mathbf{B}=$ biopsy, $\mathbf{f}=$ female, $\mathbf{m}=$ male, $\mathbf{R R}=$ relapsing remitting, $\mathbf{n s}=$ not specified

\subsection{Animals}

For in vivo experiments, adult female inbred 2-3 month old Lewis rats (180-245 g) were purchased from Charles River (Sulzfeld, Germany).

For isolation of astrocytes and endothelial cells newborn Wistar rats were used on postnatal day 0 (P0) or P17, respectively. They were obtained from the in-house breeding facility.

Animals were kept in cages with up to 5 animals each on a $12 / 12 \mathrm{~h}$ light/dark cycle at constant temperature and humidity with access to food and water ad libitum. Before each experiment, the animals were allowed to adapt to the new environment for 7 days. All animal experiments were conducted in accordance with the European Communities Council Directive of 24 November 1986 (86/EEC) and were approved by the Government of Lower Saxony, Germany (12/0964). 


\section{$2.4 \quad$ Methods}

\subsubsection{Animal experiments}

\subsubsection{Induction of focal NMO-like lesions in rats}

NMO-like lesions in rats were induced by intracortical injection of a human recombinant NMO-Ab, directed against AQP4, together with complement. The NMO-Ab was generated in the laboratory of Jeffrey Bennett (Department of Neurology and Ophthalmology, University of Colorado, Denver, USA). Briefly, cerebrospinal fluid (CSF) plasma cells were isolated from an NMO patient after a first clinical attack. The paired heavy- and light-chain sequences from a single plasma cell clone were reconstructed to create AQP4-specific recombinant human IgG1 monoclonal antibodies (Bennett et al., 2009). For the induction of focal NMO-like lesions the recombinant anti-AQP4 Ab 53 ( $\mathrm{rAB}-53$; from now on referred to as NMO-Ab) was used. A similarly produced non-CNS antigen specific recombinant human IgG1 antibody directed against measles virus nucleocapsid protein ( $\mathrm{AAb} 2 \mathrm{~B} 4$; designated in the following control-(ctrl-)Ab) served as a control.

To induce focal NMO-like lesions, female Lewis rats were anesthetized by intraperitoneal (i.p.) injection of ketamine $(60 \mathrm{mg} / \mathrm{kg}$ body weight) and xylazine $(8 \mathrm{mg} / \mathrm{kg}$ body weight). After loss of consciousness and pedal reflexes, a rostro-caudal cut was performed to gain access to the skull. The head of the animal was fixed in a stereotactic device and a small hole was drilled through the skull, $1 \mathrm{~mm}$ caudally and $2 \mathrm{~mm}$ laterally to the bregma. When only a thin layer of bone was left, a small hole was made using a microdissection knife in order to avoid damage to the brain. Using a finely calibrated glass capillary, $1 \mu l$ antibody mixture was slowly injected into the cortex. This mixture contained $2.5 \mathrm{mg} / \mathrm{ml} \mathrm{Ab}$ (either NMO-Ab or ctrlAb), $15 \mathrm{U} / \mathrm{ml}$ human complement and the tracer dye Monastral Blue. After injection, the capillary was carefully removed and the skin was sutured. To provide analgesia $0.03 \mathrm{mg} / \mathrm{kg}$ buprenorphine (Temgesic(C) was injected i.p. directly after surgery, which was repeated after $6 \mathrm{~h}$ and $12 \mathrm{~h}$.

\subsubsection{Assessment of blood brain barrier (BBB) permeability}

In order to assess the integrity of the $\mathrm{BBB}$, the extravasation of the endogenous marker molecules IgG and fibrinogen was investigated by immunohistochemical staining. Additionally, experiments applying exogenous tracers were performed. Therefore, two differently sized, lysine-fixable marker molecules were used: fluorescein isothiocyanate (FITC)-albumin (70 kDa) and Texas $\operatorname{Red}^{\circledR}$ cadaverine (690 Da). Rats were intravenously (i.v.) 
injected either with $1 \mathrm{ml}$ of a $10 \mathrm{mg} / \mathrm{ml} \mathrm{FITC-albumin} \mathrm{solution} \mathrm{(dissolved} \mathrm{in} \mathrm{saline)} 40 \mathrm{~min}$ before perfusion, or $1 \mathrm{ml}$ of a $5 \mathrm{mg} / \mathrm{ml}$ Texas Red cadaverine solution (dissolved first in $40 \mu \mathrm{l}$ DMSO, and then filled up to $1 \mathrm{ml}$ with saline solution) $50 \mathrm{~min}$ before perfusion.

\subsubsection{Depletion of polymorphonuclear cells (PMN) in rats}

For the depletion of blood PMN in rats rabbit anti-PMN antiserum (Accurate Chemical and Scientific Corporation) was used. Control rats received normal rabbit serum (Accurate Chemical and Scientific Corporation). To deplete PMN $1 \mathrm{ml} / \mathrm{kg}$ body weight anti-PMN antiserum or normal serum (filled to $2 \mathrm{ml}$ with saline) was injected i.p. $18 \mathrm{~h}$ prior and directly after intracerebral injection of NMO- or ctrl-Ab. To verify the efficiency of PMN depletion, blood samples were taken twice from the animals: before PMN depletion and at $6 \mathrm{~h}$ after lesion induction when the animals were sacrificed. Blood smear samples were analyzed by Giemsa staining. Therefore, air dried blood smears were incubated in a 1:5 dilution of Giemsa's azur-eosin-methylene blue solution for $40 \mathrm{~min}$. Subsequently, the staining was differentiated in $1 \% \mathrm{HCl}$ (in distilled water) and the smear was dehydrated and mounted. Differential cell counts were performed using light microscopy (400 x magnification). In each slide, 300 cells were counted and the percentage of PMN was calculated.

In addition, the effect of the treatment with anti-PMN antiserum on PMN, B-cells, T-cells and monocytes/macrophages was investigated using flow cytometry. Flow cytometric analysis was performed by Michael Haberl (Department of Neuroimmunology, Institute for Multiple Sclerosis research, Göttingen). Blood samples were obtained by cardiac puncture once the animals were sacrificed, and diluted 1:1 with PBS. Subsequently, 0.75 volumes $20 \%$ dextran ( $\geq 450 \mathrm{kDa}$ ) were added to the blood/PBS mixture and incubated at room temperature (RT) for $30 \mathrm{~min}$ in order to allow for the aggregation of erythrocytes at the bottom of the tube. The leukocyte-rich plasma was then transferred to a new tube and centrifuged at $1200 \mathrm{rpm}$ for 5 min. The supernatant was discarded, the pellet was washed with PBS and centrifuged again. Subsequently, the cell pellet was resuspended in FACS buffer. $100 \mu \mathrm{l}$ of the cell suspension were used for each staining. Prior to granulocyte labeling, cells were fixed with 2\% PFA for 20 min, centrifuged, washed with FACS-buffer and centrifuged again. The cells were then permeabilized by resuspension of the cell pellet in $1 \mathrm{x}$ BD Perm/Wash ${ }^{\mathrm{TM}}$ buffer, incubated for 30 min on ice and washed twice with FACS-buffer. For staining of CD4 T cells, CD8 T cells, B cells and myeloid cells, no pretreatment was needed. Subsequently, Ab were diluted 1:100 in FACS buffer, added to the cells and incubated on ice in the dark for 30 min. The following Ab were used: granulocytes: RP-1; CD4 T cells: TCR $\alpha / ß$ and CD4; CD8 T cells: 
TCR $\alpha / \beta$ and CD8a; $B$ cells: CD45RA; myeloid cells: CD11b. Cells were washed twice in FACS buffer and the cell pellet was resuspended in $100 \mu$ l FACS buffer. Finally, $50 \mu$ of BD Calibrite $^{\mathrm{TM}}$ beads $\left(20 \times 10^{4}\right.$ beads $\left./ \mathrm{ml}\right)$ were added to the suspension and cells were counted until a minimum 1000 bead events were recorded using the BD FACSCalibur.

\subsubsection{Pharmacological inhibition of the complement component 5a receptor (C5aR)}

To inhibit C5aR mediated signaling, the small molecule C5aR antagonist PMX-53 was used. PMX-53 was dissolved in distilled water at a concentration of $10 \mathrm{mg} / \mathrm{ml}$ and then further diluted in normal sterile saline $(0.9 \%)$ to a final concentration of $0.2 \mathrm{mg} / \mathrm{ml}$.

Animals were treated once i.v. with $1 \mathrm{mg} / \mathrm{kg}$ body weight PMX-53 or vehicle $3 \mathrm{~h}$ after focal intracerebral injection of NMO-Ab and human complement. $6 \mathrm{~h}$ after focal injection rats were perfused and brains were prepared for histological analysis.

\subsubsection{Pharmacological inhibition of neutrophil elastase}

For inhibition of the neutrophil elastase (NE), the competitive NE inhibitor Sivelestat sodium salt hydrate was used. Sivelestat was dissolved to a final concentration of $20 \mathrm{mg} / \mathrm{ml}$ in water. Rats were injected i.p. with $50 \mathrm{mg} / \mathrm{kg}$ body weight Sivelestat or vehicle $3 \mathrm{~h}$ after NMO-like lesion induction. Rats were perfused $6 \mathrm{~h}$ after intracerebral injection of NMO-Ab and human complement, and brains were processed for histological investigation.

\subsubsection{Perfusion of animals}

In order to preserve the tissue morphology for histological analyzes and to remove the blood from the vasculature, animals were perfused transcardially. Rats were anesthetized by i.p. injection of a lethal dose of $14 \%$ chloral hydrate. After the animal was deeply sedated and had lost the pedal and corneal reflexes, the thorax was opened. The perfusion was performed via the left heart ventricle.

For histological evaluations, animals were perfused with PBS followed by 4\% PFA. Subsequently, the brain, spleen and liver were collected in a falcon tube containing 4\% PFA and were post-fixed for an additional $48 \mathrm{~h}$ at $4^{\circ} \mathrm{C}$. The brain was then washed with PBS, dissected into 2-4 $\mathrm{mm}$ thick coronal sections close to the injection site marked with Monastral blue and washed again with water. Spleen and liver were processed in the same way and served as ctrl tissue in the same paraffin block. For paraffin embedding, the tissues were gradually dehydrated via alcohol, xylene and in the end embedded in paraffin using the automated tissue processor TP 1020 over night. 
For RNA extraction and cryo-tissue preparation the perfusion was performed with sterile PBS only. After PBS perfusion the brain was removed, trimmed near the injection site and placed in a drop of Tissue-Tek ${ }^{\circledast}$ on a cork plate. Subsequently, the tissue was frozen in liquid nitrogen-cooled isopentan and stored at $-80^{\circ} \mathrm{C}$.

\subsubsection{Cell culture}

\subsubsection{Isolation and purification of primary rat brain endothelial cells (RBEC) and measurement of the transendothelial resistance (TEER)}

Primary RBEC were isolated based on a modified protocol published by Joan Abbott and coworkers in 2006 (Abbott et al., 2006). For the isolation, 17 days old Wistar rats were used. Rats were deeply anesthetized with isoflurane and decapitated. Brains were removed and directly transferred into dissection buffer in which they were stored on ice until further processing. All the following steps were performed under sterile conditions in a laminar flow hood. From each brain, the cerebellum, the olfactory bulbs and the optic nerves were removed, and both hemispheres were separated. One hemisphere was transferred to Whatman filter paper, the midbrain was removed, and the brain was folded open in order to remove the white matter (WM) and meninges. Subsequently the grey matter (GM) tissue was collected and transferred to a new Petri dish. The GM was cut into small pieces and transferred to a new tube containing dissection buffer and stored on ice. After centrifugation $\left(800 \times \mathrm{g}, 4^{\circ} \mathrm{C}, 7 \mathrm{~min}\right) 7 \mathrm{ml}$ of digestion solution containing Collagenase $\mathrm{D}$, Dispase and DNase I was added to the pellet. The tissue was digested at $37^{\circ} \mathrm{C}$ for 30 min under constant agitation and then homogenized by pipetting. The suspension was centrifuged, the supernatant was aspirated and $20 \mathrm{ml}$ of ice cold DMEM with $20 \%$ BSA was added. The pellet was thoroughly resuspended and centrifuged at $1000 \mathrm{xg}$ for $20 \mathrm{~min}$ at $4^{\circ} \mathrm{C}$. After centrifugation the upper phase contains myelin, oligodendrocytes, neurons and microglia, while microvessels can be found as a pellet at the bottom of the tube. The supernatant was discarded and the microvessels were washed once with dissection buffer and subsequently resuspended in 3 $\mathrm{ml}$ digestion buffer. After an additional digestion time of $20 \mathrm{~min}$ at $37^{\circ} \mathrm{C}$ the cells were centrifuged. The pellet was resuspended in selection medium containing puromycin in order to remove contaminating non-P-glycoprotein-expressing cells (Perrière et al., 2005). RBEC were seeded in 12 well inserts (PET membrane, $0.4 \mu \mathrm{m}$ pore size) precoated with collagenIV and fibronectin and placed in a $\mathrm{CO}_{2}$ incubator $\left(5 \% \mathrm{CO}_{2}, 37^{\circ} \mathrm{C}\right)$.

After incubation for $3 \mathrm{~d}$, selection medium was replaced with normal growth medium. The medium was replaced every other day and exchanged by experimental medium containing 
hydrocortisone once the RBEC layer had reached $90 \%$ confluency. Cells were directly used for experiments when they reached TEER values of around $40 \Omega^{*} \mathrm{~cm}^{2}$.

TEER measurements are an important readout to assess the integrity of the RBEC monolayer. It represents the permeability of tight junctions (TJ) to sodium ions. High TEER values represent an impermeable monolayer. TEER was measured using the EVOM ${ }^{2}$ resistance meter (World Precision Instruments) with STX-2 electrodes, where one electrode is placed in the apical and the other one in the basolateral compartment. The resistance measured was then expressed relative to the surface area of the insert $\left(\Omega^{*} \mathrm{~cm}^{2}\right)$. The TEER values of coated cell-free inserts $\left(140-155 \Omega^{*} \mathrm{~cm}^{2}\right)$ was subtracted from the values of the monolayers.

To ensure that treatment with puromycin removed all contaminating cells from the RBEC culture, initial qPCR for AQP4 (astrocytes), CD11b (microglia), $\alpha-S M A$ and PDGFRß (pericytes), and immunocytochemistry for endothelial cell markers (VWF, occludin, claudin-3, claudin-5) was performed. qPCR and immunohistochemistry showed no contamination by other cells.

\subsubsection{Isolation and purification of primary rat astrocytes}

Primary rat astrocytes were isolated from newborn Wistar rats (P0-P1). Rats were decapitated, the brain was isolated and transferred into a Petri dish containing HBSS buffer $\left(37^{\circ} \mathrm{C}\right)$. Subsequently, the meninges were removed with forceps to prevent meningeal fibroblasts to interfere with the glial cell growth. Brains were digested in $0.25 \%$ Trypsin-EDTA solution for $20 \mathrm{~min}$ at $37^{\circ} \mathrm{C}$. Then the cells were washed once with HBSS. Subsequently, DMEM containing $10 \%$ FCS and $1 \% \mathrm{P} / \mathrm{S}$ was added to the tissue pellet and the tissue was dissociated by pipetting. Cells were seeded in PLL coated falcon flasks and grown for 10 to 14 $\mathrm{d}$ in an incubator $\left(5 \% \mathrm{CO}_{2}, 37^{\circ} \mathrm{C}\right)$ with medium changes every $2-3 \mathrm{~d}$. When the mixed cell culture containing astrocytes, oligodendrocytes and microglia reached $100 \%$ confluency, the loosely sitting oligodendrocytes and microglia were removed by vigorous shaking of the flasks. The supernatant was discarded, while astrocytes stayed firmly attached to the plastic surface. Astrocytic cultures were used maximally for 3 weeks to avoid loss of primary phenotype.

\subsubsection{Co-culture of RBEC and astrocytes as in vitro model of the BBB}

In order to assess the indirect influence of astrocytes on RBEC, cells were co-cultured in a 12well transwell system. Astrocytes were plated in a 12-well cell culture plate (basolateral 
compartment) containing astrocyte medium. At the same time, RBEC were seeded in inserts (apical compartment). When astrocytes reached $100 \%$ confluency, inserts with a confluent RBEC monolayer were transferred into the 12-well plate containing astrocytes. Astrocyte medium was replaced by RBEC experimental medium. TEER was measured daily for $7 \mathrm{~d}$ in triplicates.

\subsubsection{Incubation of RBEC monolayers with proteases and assessment of cytotoxicity}

To investigate the direct influence of neutrophil proteases on RBEC, NE $(6 \mu \mathrm{g} / \mathrm{ml})$, cathepsin $\mathrm{G}(0.4 \mathrm{U} / \mathrm{ml})$ or active matrix metalloproteinase $9(0.5 \mu \mathrm{g} / \mathrm{ml})$ were added directly to the apical compartment of a transwell system containing RBEC. Experiments were performed in triplicates. TEER was measured before treatment and after 60 and $120 \mathrm{~min}$. Subsequently, cells were fixed with $100 \%$ ethanol for immunocytochemical analysis.

To exclude a cytotoxic effect of neutrophil proteases on RBEC the CytoTox-ONE ${ }^{\mathrm{TM}}$ Homogeneous Membrane Integrity Assay (Promega) was used according to the manufacturer's instructions. It is based on the release of lactate dehydrogenase from dying cells with a leaky cell membrane. The lactate dehydrogenase-measurement was performed after $2 \mathrm{~h}$ of treatment with proteases using $100 \mu \mathrm{l}$ of the supernatant from the apical compartment. Supernatant of lyzed RBEC served as positive control as described in the manufacturer's protocol.

\subsubsection{Immunocytochemcial staining of RBEC}

To investigate TJ expression and localization in the isolated RBEC, cells were grown on coverslips. After reaching confluency, they were washed once with warm PBS, transferred into precooled $100 \%$ ethanol and fixed at $-20^{\circ} \mathrm{C}$ for at least $30 \mathrm{~min}$. Coverslips were washed 3 times with PBS and incubated with blocking buffer (PBS with $10 \%$ horse serum and $1 \%$ BSA) for $20 \mathrm{~min}$. Primary antibody diluted in blocking buffer was added to the coverslips for $1 \mathrm{~h}$. RBEC were immunolabeled for the TJ molecules occludin (1:50), claudin-3 (1:100), claudin-5 (1:300) and VWF (1:100) (Table 5). Cells were washed 3 times with PBS and subsequently incubated with a fluorescently labeled secondary antibody (Table 6) diluted in blocking buffer for $30 \mathrm{~min}$. Cells were washed and the nuclei were stained using 4',6-diamidino-2phenylindole (DAPI, 1:10000 in PBS). After a last washing step coverslips were rinsed in distilled water and mounted on glass slides using fluorescence mounting medium. 


\subsubsection{Histology}

\subsubsection{Cutting, deparaffinization and dehydration of histological paraffin- embedded sections}

Paraffin blocks were cut into 1-2 $\mu \mathrm{m}$ thick sections using a sliding microtome, and were then mounted on glass slides. For identification of the injection sites, sections were checked for traces of Monastral blue in the tissue.

Prior to the staining procedure, sections were gradually deparaffinized. Therefore, the paraffin was melted for at least $1 \mathrm{~h}$ at $56^{\circ} \mathrm{C}$. Subsequently, the sections were first delipidized and then rehydrated by consecutive immersion in the following solutions: xylene (four times for $5 \mathrm{~min}$ ), isoxylene (once for $2 \mathrm{~min}$ ), $100 \%$ isopropyl alcohol (twice for $3 \mathrm{~min}$ ), and once in $90 \%, 70 \%$ and $50 \%$ isopropyl alcohol for $3 \mathrm{~min}$ each, and in the end washed with distilled water.

After the staining, sections were again dehydrated in reverse order to the deparaffinization procedure and mounted in DePex medium.

\subsubsection{Histochemical stainings}

\subsection{Hematoxylin and eosin staining (HE)}

For a general overview of the tissue with regard to inflammation and apoptosis HE staining was performed. The first component of the staining is hematoxylin, a basic dye which colors basophilic structures (particularly the DNA in the nucleus and the rough endoplasmic reticulum) with a blue-purple hue. The second component is the alcohol-based acidic eosin that stains eosinophilic structures like proteins bright pink. For staining, the sections were deparaffinized, washed and incubated in Mayer's hemalum for $8 \mathrm{~min}$. Then slides were washed with water, briefly differentiated in $1 \% \mathrm{HCl}$ and blued for $10 \mathrm{~min}$ in running tap water. Subsequently, the slides were stained with $1 \%$ eosin for $6 \mathrm{~min}$, washed with distilled water, dehydrated and mounted.

\subsection{Bielschowsky silver impregnation}

In order to ensure that the stereotactic injections into the brain were not destroying the surrounding tissue reflected by axonal destruction and tissue necrosis, Bielschowsky silver impregnation was used. It stains nerve fibers and pathological deposits black. Sections were deparaffinized and incubated in $20 \%$ silver nitrate (in distilled water) for 20 min and subsequently transferred into a cuvette with distilled water. Then $32 \%$ ammonium hydroxide was added dropwise into the $20 \%$ silver nitrate solution until the resulting 
precipitations were cleared. Slides were returned into the cuvette containing silver nitrate and ammonium hydroxide and incubated in the dark for $15 \mathrm{~min}$. Slides were then placed in $50 \mathrm{ml}$ distilled water containing three drops of ammonium hydroxide. Now 10 drops of developing solution were added to the silver nitrate and ammonium hydroxide solution and slides were briefly developed. When the desired staining intensity was reached, the reaction was stopped in distilled water. Slides were then incubated for $2 \mathrm{~min}$ in $2 \%$ sodium thiosulfate, rinsed, dehydrated and mounted.

\subsection{Chloroacetate esterase enzyme histochemistry (CAE)}

CAE stain (also called Leder stain) takes advantage of the enzymatic activity of "specific esterase", an enzyme found in cells of the granulocyte lineage and was therefore used to detect neutrophils in the brain. In the naphthol AS-D chloroacetate kit (Sigma) that was used, naphthol AS-D chloroacetate is enzymatically hydrolyzed. Thereby a naphtol compound is liberated and couples with a diazonium compound, leading to the formation of bright pink deposits at sites of enzymatic activity.

CAE staining was performed according to manufacturer's instruction. Briefly, the slides were deparaffinized, rinsed in distilled water and preheated in PBS at $37^{\circ} \mathrm{C}$ for $1 \mathrm{~h}$. Then the working solution was prepared, consisting of $2 \%$ sodium nitrite, $2 \%$ Fast Corinth salt, $10 \%$ Trizmal $^{\mathrm{TM}}$ buffer and $2 \%$ naphtol AS-D chloroacetate. Slides were then incubated in the working solution at $37^{\circ} \mathrm{C}$ for $2 \mathrm{~h}$ and washed with distilled water. Subsequently they were briefly counterstained in Mayer's hemalum, washed and mounted using Aquamount.

\subsubsection{Immunohistochemistry}

\subsection{Antigen retrieval of paraffin embedded tissues}

Due to fixation of the tissues with PFA, antigens in the sections can be masked. This can prevent the primary $A b$ binding to the antigen. Therefore we used different methods to unmask the epitopes, which were determined separately for each antibody and are listed in Table 5.

For heat-induced antigen retrieval 10mM citric acid, $1 \mathrm{mM}$ Tris-EDTA or Tris- $\mathrm{HCl}$ solutions were used. The sections were heated in a microwave 5 times for $3 \mathrm{~min}$, refilling first with water and then alternatingly with buffer and water after each cycle. After the solutions cooled down, buffers were replaced by water. 
Alternatively, proteinase K-induced antigen retrieval was used. Here the slides were incubated at $60^{\circ} \mathrm{C}$ in a preheated proteinase $\mathrm{K}$ solution for $10 \mathrm{~min}$ and then washed with water.

\subsection{Cutting and fixation of frozen tissues}

Native, unfixed frozen tissues were cut into $5 \mu \mathrm{m}$ thick sections using a cryostat and mounted on glass slides. They were then either stored at $-80^{\circ} \mathrm{C}$ or further processed. To prevent detachment of the sections from the slides during the staining procedure, the sections were air-dried for at least $30 \mathrm{~min}$ at RT.

Frozen tissues were fixed depending on the antibody, either for $10 \mathrm{~min}$ in $100 \%$ ethanol or $30 \mathrm{~min}$ in $100 \%$ ether at RT (see Table 5). Subsequently sections were air-dried for $10 \mathrm{~min}$, rehydrated for $10 \mathrm{~min}$ in PBS and immunostained.

\subsection{Immunostaining of tissues}

Immunostainings were performed in order to investigate astrocyte pathology (GFAP, AQP4), BBB breakdown (IgG, Fibrinogen, FITC, Texas Red), immune cell infiltration (ED1, CD3, Iba1, MRP14, KIM1P), blood vessel pathology (occludin, claudin-3, claudin-5, LAMY1) or pericytes (PDGFRß).

For immunohistochemical staining, the labeled streptavidin-biotin method was used. To block the endogenous peroxidase, pretreated frozen or paraffin embedded tissues were first incubated with $3 \%$ hydrogen peroxide in PBS and then washed 3 times in PBS. To block unspecific antibody binding, sections were treated with blocking buffer (PBS with 10\% FCS) for $10 \mathrm{~min}$. Next, the primary antibody, diluted in blocking buffer (Ab dilutions are given in Table 5) was added and incubated over night at $4^{\circ} \mathrm{C}$. Afterwards, slides were rinsed 3 times with PBS. Then the corresponding biotinylated secondary antibody, diluted in blocking buffer, was applied to the slides for $1 \mathrm{~h}$ at RT (Table 6). Non-bound antibody was removed by washing 3 times with PBS. To amplify the signal $0.1 \%$ streptavidin horseradish peroxidase diluted in blocking buffer was added to the slides and incubated for $45 \mathrm{~min}$ at RT. After washing 3 times with PBS, slides were developed in a 3,3' diaminobenzidine (DAB) solution, and the reaction was controlled under a microscope. The chromogen DAB acts here as a substrate of the peroxidase, which results in a dark brown staining. Subsequently, the slides were washed in distilled water and counterstained in Mayer's hemalum for $30 \mathrm{~s}$. Then they were washed with distilled water, differentiated in $1 \% \mathrm{HCl}$ solution, blued in tap water for 7 min, dehydrated and mounted as described above. 
The indirect immunofluorescence method was used in order to stain different target antigens simultaneously, e.g. to evaluate the TJ of vessels within the lesions. Pretreated frozen tissues were washed 3 times with PBS, blocked with blocking buffer and incubated with the primary antibodies (Table 5 ) at $4^{\circ} \mathrm{C}$ over night. Subsequently slides were washed 3 times in PBS and incubated with the corresponding fluorochrome-labeled secondary antibodies (Table 6) for 45 min at RT in the dark. After washing with PBS 3 times, nuclei were counterstained with DAPI (1:10000 in PBS) for $15 \mathrm{~min}$ at RT in the dark. Then the slides were washed again 3 times in PBS, rinsed once in distilled water and mounted using fluorescence mounting medium.

\subsubsection{Electron microscopy (EM)}

For assessment of the TJ morphology on the ultrastructural level, electron microscopy (EM) was performed. Therefore animals were perfused first with PBS and subsequently with $4 \%$ PFA. Then the brain was removed and cut coronally at the injection site. For postfixation the area around the injection site was cut into three pieces $\left(1 \mathrm{~mm}^{3}\right)$ which were then transferred to $3 \%$ glutaraldehyde over night. The brain pieces were washed two times in PBS and incubated with $1 \%$ osmium tetroxide solution for $1 \mathrm{~h}$ at $4^{\circ} \mathrm{C}$. Dehydration followed for $10 \mathrm{~min}$ in $50 \%$ ethanol, and additional staining was performed with $0.5 \%$ uranyl acetate for $1 \mathrm{~h}$ at $4{ }^{\circ} \mathrm{C}$ in the dark. The tissue was dehydrated in $70 \%$ ethanol over night, for $10 \mathrm{~min}$ in $80 \%$ and $96 \%$ ethanol and two times for $15 \mathrm{~min}$ in $100 \%$ ethanol. Next, for resin embedding, the tissue was incubated 2 times in propylene oxide for $20 \mathrm{~min}$. Afterwards resin:propylene oxide ratios were adjusted to $1: 1$ and subsequently to $2: 1$ solution in which the tissue was incubated for $35 \mathrm{~min}$ each. Finally, the tissue was transferred into $100 \%$ resin for $1 \mathrm{~h}$ and embedded in moulds filled with araldite.

Embedded tissues were cut in semi-thin sections of $360-400 \mathrm{~nm}$. Sections were transferred to a glass slide and dried on a heating plate at $60{ }^{\circ} \mathrm{C}$ for $1 \mathrm{~h}$. Semi-thin sections were stained with Richardson's stain, which stains myelin in dark blue and cytoplasm in light blue, for 3 min. Finally they were rinsed 3 times with distilled water air dried and mounted with Depex. According to the area of interest in the semi-thin section, the tissue blocks were trimmed and ultrathin sections of 70-72 $\mathrm{nm}$ were cut. These sections were collected on a grid, dried and stained with lead citrate for $10 \mathrm{~min}$.

All samples were evaluated using the electron microscope EM10B (Zeiss) and imaged. 


\subsubsection{Microdissection of lesions for mRNA expression analysis}

In order to evaluate mRNA expression of TJ genes in NMO-like lesions, laser capture microdissection (LCM) was performed. Therefore brain tissue of NMO- or ctrl-Ab injected animals was fixed in liquid nitrogen. Tissue was cut into $10 \mu \mathrm{m}$ thick sections using a cryostat, mounted on polyethylene naphthalate membranes and stored at $-80^{\circ} \mathrm{C}$.

In order to prevent mRNA degradation, a rapid staining protocol for astrocytes was established to identify the lesion outline. The tissue was first fixed in $-20^{\circ} \mathrm{C}$ cold ethanol for 2 min, washed briefly with PBS and then stained for 2 min with anti-GFAP-Cy3. Subsequently slides were washed with PBS and dipped into $100 \%$ ethanol for dehydration. LCM was then performed using the MMI CellCut Plus system microscope. In order to prevent RNA degradation LCM was performed for a maximum of $10 \mathrm{~min}$. Only astrocyte depleted areas were extracted from NMO-Ab injected animals, while in ctrl-Ab injected animals the area around the injection site was isolated. The extracted tissue was directly transferred into RLTbuffer (according to the manufacturer's instructions containing 1\% ß-mercaptoethanol) and stored at $-80^{\circ} \mathrm{C}$ until further processing.

\subsection{5 mRNA expression analysis}

RNA was isolated using the RNeasy Micro Kit (Qiagen) according to the manufacturer's protocol for microdissected tissues. Subsequently, RNA was transcribed into cDNA using the High-Capacity RNA-to-cDNA ${ }^{\mathrm{TM}}$ Kit (Life Technologies) according to the manufacturer's instructions.

qPCR was performed using the qPCR core kit (Eurogentec) according to the manufacturer's instructions. TaqMan Gene Expression Assays (Life Technologies) for claudin-3, -5 and occludin (TJ molecules) were used. The house keeping gene gapdh served as control. Duplicates of each probe were checked for pipetting errors which would result in high standard deviation (SD). Samples with a SD higher than 0.4 were excluded from further calculation.

\subsubsection{Morphometric analysis and data acquisition}

Astrocyte lesion size and extravasation of molecules into the parenchyma was measured using ImageJ. Therefore, images were taken at 40x magnification using the BX51 Olympus light microscope equipped with a DP71 digital camera. 
For assessment of lesion size, areas of complete loss of AQP4 and GFAP immunoreactivity were measured.

For extravasation of the endogenous tracer molecules IgG and fibrinogen and the exogenous tracer molecules FITC-albumin and Texas $\operatorname{Red}^{\circledR}$-cadaverine the different channels (haematoxylin-DAB-3rd component) were separated using the ImageJ plugin DAB color deconvolution. In the brown channel (DAB) the intensity threshold for selection was set to 0150 and the resulting marked area was measured. For the IgG and fibrinogen time course the intensity threshold was adjusted to the staining intensity due to background differences in the different slides.

To evaluate the percentage of blood vessels positive for TJ molecules, immunofluorescently labeled sections (NMO-Ab injected: claudin-3/claudin-5/occludin - LAMY1 - GFAP; ctrl-Ab injected: claudin-3/claudin-5/occludin - LAM 1 1) were imaged at 100x magnification using the BX51 Olympus light microscope equipped with a XM10 monochrome camera. The total number of LAMY1-positive blood vessels was evaluated as well as the number of LAMY1positive blood vessels which were immunopositive for occludin, claudin-3 or claudin-5 at the TJ. From this, the percentage of brain vessels with occludin, claudin-3 or claudin-5 localized at the TJ was calculated.

To assess the number and density of immune cells infiltrating focal NMO-like lesions, immunohistochemistry for ED1 (monocytes/macrophages) and CD3 (T cells) was performed. In addition, CAE was used to label PMN. Cells were counted using a light microscope with an ocular morphometric grid. For assessment of infiltrates in animals, cells were counted in the whole lesion at 100x magnification. For human lesions the density of immune cells was evaluated by evaluating the total number of positive cells in 5 visual fields at $400 x$ magnification.

\subsubsection{Statistical analysis}

Statistical analysis was carried out using the GraphPad Prism 5.01 software.

For parametric data the independent t-test with Welch's correction was used to compare two groups. To compare more than two groups, the Kruskal-Wallis test followed by Dunn's multiple comparison test was performed. Pearson's correlation coefficients were used to assess associations between area of FITC-albumin extravasation and number of infiltrated $P M N$. Statistical significance was defined as $p<0.05$. All data are given as mean $\pm S D$. 


\subsection{Induction of neuromyelitis optica (NMO)-like lesions results in breakdown of the blood-brain barrier (BBB)}

\subsubsection{Time course of astrocyte depletion after focal injection of NMO-antibody and human complement}

To investigate the pathogenesis of lesion formation in NMO, a focal model of NMO-like lesions in Lewis rats was established in our group (Wrzos et al., 2014). In this model NMOlike lesions were induced by stereotactic injection of a recombinant human NMO-antibody (Ab) directed against aquaporin4 (AQP4) together with human complement into the motor cortex of Lewis rats (Bennett et al., 2009). As a control, stereotactic injection of a similarly produced non-CNS antigen specific control (ctrl)-Ab together with human complement was performed. To investigate the development of the astrocyte depleted lesion, a time course study was done. Therefore, the animals were sacrificed $3 \mathrm{~h}, 6 \mathrm{~h}, 24 \mathrm{~h}, 3 \mathrm{~d}$ and $7 \mathrm{~d}$ after lesion induction (Figure $3 \mathrm{~A}$ ). Lesion development was investigated using immunohistochemistry for the astrocyte markers glial fibrillary acidic protein (GFAP) and AQP4.

$3 \mathrm{~h}$ after lesion induction no confluent loss of GFAP immunoreactivity was observed (Figure 3 B). However, damaged GFAP positive cells with retracted cell processes were detected (Figure $3 \mathrm{~B}$, insert). Confluent, well-demarcated areas of GFAP loss were observed $6 \mathrm{~h}$ after lesion induction (area of GFAP loss: $0.26 \pm 0.15 \mathrm{~mm}^{2}$, Figure $3 \mathrm{G}$ ) in contrast to ctrl-Ab injected animals at the same time point $\left({ }^{*} p<0.05\right)$. Subsequently, loss of GFAP immunoreactivity expanded until lesions developed the maximal extent between $24 \mathrm{~h}$ and $3 \mathrm{~d}$ (area of GFAP loss: $24 \mathrm{~h}=1.03 \pm 0.89 \mathrm{~mm}^{2}$ and $3 \mathrm{~d}=1.11 \pm 0.85 \mathrm{~mm}^{2},{ }^{*} \mathrm{p}<0.5, * * \mathrm{p}<0.01$, $* * * p<0.001$, Figure $3 \mathrm{G}$ ). Loss of GFAP immunoreactivity in astrocyte depleted lesions was confirmed by loss of AQP4 immunoreactivity $24 \mathrm{~h}$ after lesion induction (Figure $3 \mathrm{C}, \mathrm{D}$ ). No loss of GFAP- (Figure $3 \mathrm{E}$ ) and AQP4-positive cells (Figure $3 \mathrm{~F}$ ) was observed in animals injected with ctrl-Ab and human complement after $24 \mathrm{~h} .7 \mathrm{~d}$ after lesion induction $91.1 \%$ of the formerly astrocyte-depleted area was repopulated by GFAP positive cells (area of GFAP loss at $7 \mathrm{~d}$ : $0.09 \pm 0.04 \mathrm{~mm}^{2}$ compared to $24 \mathrm{~h}: 1.03 \pm 0.89 \mathrm{~mm}^{2}$, Figure $3 \mathrm{G}$ ). 


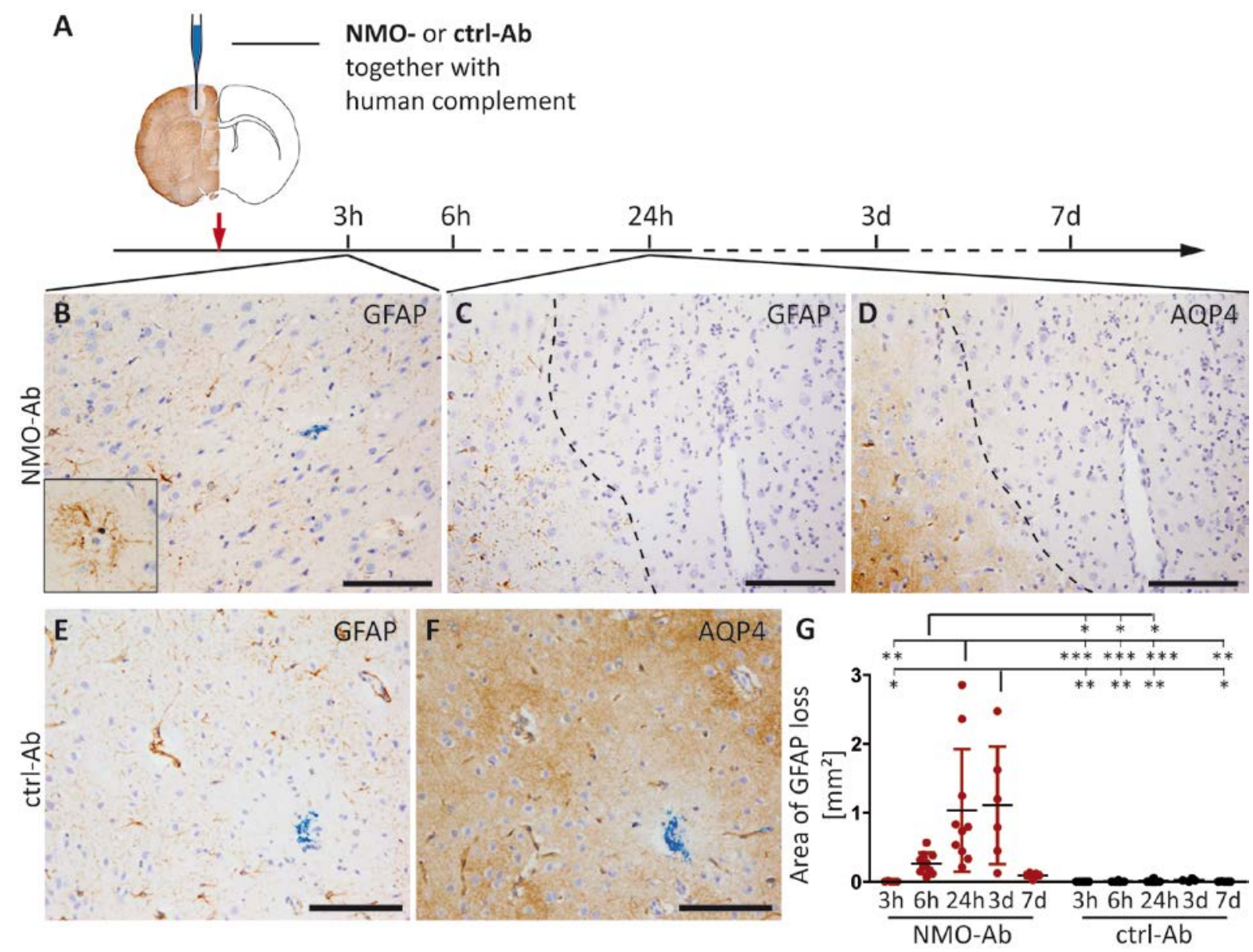

Figure 3: Injection of NMO-Ab and human complement resulted in astrocyte loss

Schematic illustration of the experimental setup for the assessment of astrocyte lesion development over time (A). $3 \mathrm{~h}$ after focal injection of NMO-Ab and human complement, GFAP positive astrocytes were still localized at the injection site (B). However, GFAP positive dying cells with retracted processes were observed (B, insert). $24 \mathrm{~h}$ after injection large, well-demarcated areas with loss of GFAP- (C) and AQP4 immunoreactivity (D; serial sections of the same lesion) were detected. No loss of GFAP (E) and AQP4 (F) positive cells was observed in animals with focal injection of ctrl-Ab and human complement after $24 \mathrm{~h}$. The time course study revealed the first loss of GFAP immunoreactivity $6 \mathrm{~h}$ after lesion induction. Astrocyte depleted lesions reached their maximal extent between $24 \mathrm{~h}$ and $3 \mathrm{~d}$. $7 \mathrm{~d}$ after focal injection $91.1 \%$ of the formerly astrocyte depleted lesion were repopulated by GFAP positive cells. No astrocyte loss was observed after injection of ctrl-Ab together with human complement (number of evaluated lesions: NMO-Ab/ctrl-Ab; 3h: $n=5 / 5 ; 6 \mathrm{~h}: n=10 / 10 ; 24 \mathrm{~h}: n=10 / 10 ; 3 \mathrm{~d}$ : $n=6 / 4 ; 7 \mathrm{~d}: n=5 / 5, \mathbf{G})$. Data were analyzed using Kruskal-Wallis test followed by Dunn's multiple comparison test and are shown as mean \pm SD. ${ }^{*} \mathrm{p}<0.05,{ }^{* *} \mathrm{p}<0.01,{ }^{* * *} \mathrm{p}<0.001$. B, E, F: Monastral blue marks the injection site. Dotted lines indicate area of astrocyte loss. Scale bar $100 \mu \mathrm{m}$

\subsubsection{Breakdown of the BBB is observed $6 \mathrm{~h}$ after lesion induction}

Astrocytes are in close proximity to brain endothelial cells (EC). In the adult brain astrocytic signaling is involved in maintaining BBB integrity (Abbott et al., 2006). To investigate whether astrocyte lesion development correlates with loss of BBB integrity, we investigated the permeability of the BBB to soluble blood-borne molecules by immunohistochemistry for immunoglobulin $\mathrm{G}(\operatorname{IgG})$ and fibrinogen. Both are plasma proteins with a size of 150 and $340 \mathrm{kDa}$, respectively. Under physiological conditions the intact BBB is impermeable to IgG 
and fibrinogen. However, under pathological conditions leakage of both proteins into the brain parenchyma may be observed.

$3 \mathrm{~h}$ after focal injection of NMO-Ab and human complement only little extravasation of IgG (area of extravasation: $0.05 \pm 0.08 \mathrm{~mm}^{2}$, Figure $4 \mathrm{~A}, \mathrm{D}$ ) and fibrinogen (area of extravasation: $0.01 \pm 0.01 \mathrm{~mm}^{2}$, Figure $4 \mathrm{E}, \mathrm{H}$ ) into the brain parenchyma was observed. However, $6 \mathrm{~h}$ after lesion induction a prominent area of the brain parenchyma immunostained positively for IgG $\left(0.38 \pm 0.34 \mathrm{~mm}^{2}\right.$, Figure $\left.4 \mathrm{~B}, \mathrm{D}\right)$ and fibrinogen $\left(0.33 \pm 0.38 \mathrm{~mm}^{2}\right.$ Figure $\left.4 \mathrm{~F}, \mathrm{H}\right)$. After $3 \mathrm{~d}$ the integrity of the BBB was restored, with some deposit-like immunopositive structures still present around the blood vessels (IgG: $0.26 \pm 0.38 \mathrm{~mm}^{2}$, Figure $4 \mathrm{C}$, G; fibrinogen: $0.11 \pm$ $0.09 \mathrm{~mm}^{2}$, Figure $4 \mathrm{G}, \mathrm{H}$ ). Measurement of the immunopositive area revealed a significant extravasation of both IgG (Figure $3 \mathrm{D}$ ) and fibrinogen (Figure $4 \mathrm{H}$ ) $6 \mathrm{~h}$ and $24 \mathrm{~h}$ after NMOlesion induction $(* p<0.5)$. Some extravasation of both proteins was also observed in animals injected with ctrl-Ab together with human complement, indicating that injection of human complement alone may result in leakage of plasma proteins from the blood vessels. However, the area of extravasation in ctrl-Ab injected animals reached only $15.7 \%(6 \mathrm{~h} \lg G)$, 15.0\% (24 h $\operatorname{lgG}$ ), 21.5\% (6 h fibrinogen) and 57.2\% (24 h fibrinogen) of the extravasation measured in NMO-Ab injected animals at the same time points (Figure $4 \mathrm{D}, \mathrm{H}$ ).

$3 \mathrm{~h}$

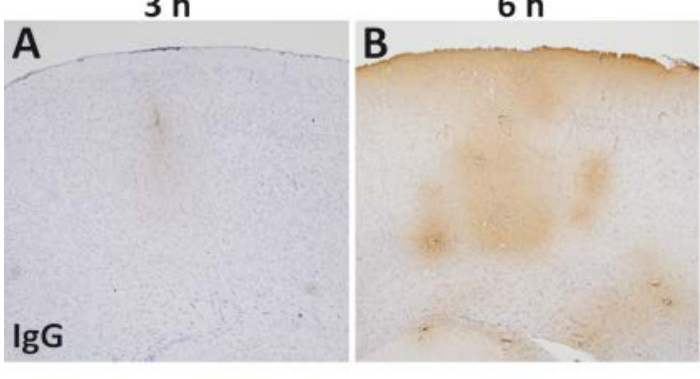

E
$3 d$
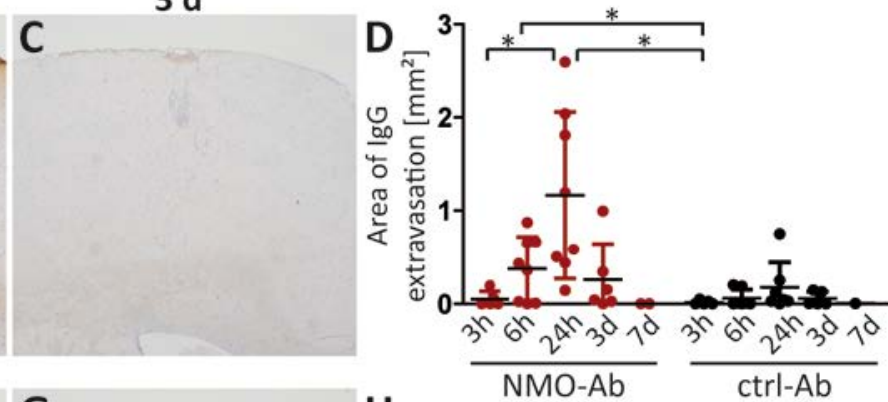

G

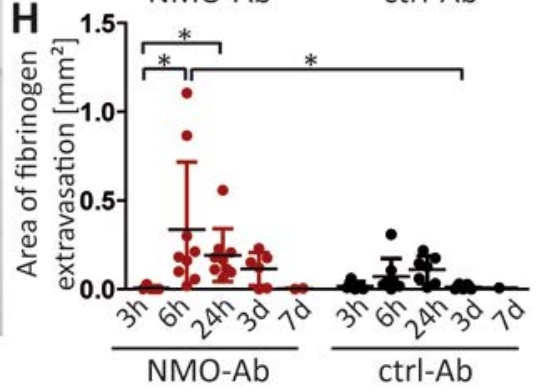

Figure 4: Breakdown of the BBB was detected $6 \mathrm{~h}$ after focal injection as assessed by immunohistochemistry for IgG and fibrinogen

$3 \mathrm{~h}$ after focal injection of NMO-Ab and human complement the BBB was intact and revealed no signs of the blood-borne proteins IgG (A) and fibrinogen (E). $6 \mathrm{~h}$ later the brain parenchyma immunostained positively for IgG (B) and fibrinogen (F) around the injection site. $3 \mathrm{~d}$ after injection the BBB appeared intact with some immunopositive deposits of IgG and fibrinogen left around the blood vessels (C, D). Quantification of the area of IgG and fibrinogen extravasation showed an accumulation of IgG and fibrinogen in NMO-Ab injected animals 
between $6 \mathrm{~h}$ and $3 \mathrm{~d}$. Extravasation in ctrl-Ab injected animals was markedly less compared to NMO-Ab injected animals. Number of lesions evaluated in D: NMO-Ab/ctrl-Ab; 3h: $n=5 / 5 ; 6 \mathrm{~h}: n=8 / 7 ; 24 \mathrm{~h}: n=8 / 7 ; 3 \mathrm{~d}$ : $n=6 / 5 ; 7 \mathrm{~d}: n=2 / 1$. Number of lesions evaluated in H: NMO-Ab/ctrl-Ab; 3h: $n=5 / 5 ; 6 \mathrm{~h}: n=9 / 8 ; 24 \mathrm{~h}: n=9 / 7 ; 3 \mathrm{~d}$ : $n=6 / 5 ; 7 \mathrm{~d}: n=2 / 1$. Data were analyzed using Kruskal-Wallis test followed by Dunn's multiple comparison test and are shown as mean \pm SD. ${ }^{*} p<0.05$. Scale bar $500 \mu \mathrm{m}$

\subsubsection{Injection of tracer molecules indicates rapid restoration of the BBB within $24 \mathrm{~h}$ after lesion induction}

Although the extravasation of endogenous, blood-borne proteins may serve as an indicator for the opening of the BBB, extravasated proteins may be retained in the brain parenchyma after the BBB integrity is re-established. To further specify the time frame of BBB disruption, the BBB permeability was additionally assessed by intravenous (i.v.)-injection of exogenous tracer molecules before perfusion. Two lysine-fixable fluorescent tracer molecules were employed: fluorescein isothiocyanate (FITC)-albumin and Texas $\operatorname{Red}^{\circledR}$ cadaverine with molecular sizes of $70 \mathrm{kDa}$ and $690 \mathrm{Da}$, respectively. Tracer molecules were injected $40 \mathrm{~min}$ (FITC-albumin) or $50 \mathrm{~min}$ (Texas $\operatorname{Red}^{\circledR}$ cadaverine) before perfusion. To analyze the permeability of the BBB, animals were stereotactically injected with NMO-Ab or ctrl-Ab and human complement. Subsequently, animals were sacrificed $3 \mathrm{~h}, 6 \mathrm{~h}, 24 \mathrm{~h}$ and $3 \mathrm{~d}$ (FITC-

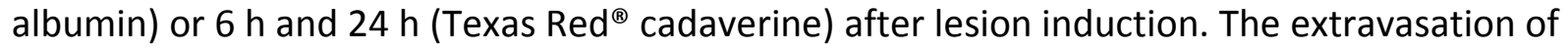
the tracer molecules was assessed by immunohistochemistry against FITC-albumin and Texas Red $^{\circledR}$ cadaverine.

$3 \mathrm{~h}$ after focal injection the BBB was impermeable to FITC-albumin (NMO-Ab: $0.00 \pm 0.00$ $\mathrm{mm}^{2}$, ctrl-Ab: $0.01 \pm 0.02 \mathrm{~mm}^{2}$, Figure $5 \mathrm{C}$ ) which confirms the observations made regarding the extravasation of the plasma proteins $\operatorname{lgG}$ and fibrinogen (Figure $4 \mathrm{D}, \mathrm{H}$ ). After $6 \mathrm{~h}$, however, a prominent extravasation of FITC-albumin (NMO-Ab: $1.03 \pm 0.54 \mathrm{~mm}^{2}$, ctrl-Ab:

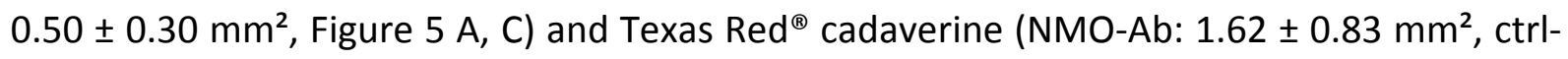
$A b: 0.84 \pm 0.38 \mathrm{~mm}^{2}$, Figure $5 \mathrm{D}, \mathrm{F}$ ) from the vasculature into the brain parenchyma was observed. $24 \mathrm{~h}$ after lesion induction the BBB integrity was again restored as demonstrated by FITC-albumin (NMO-Ab: $0.00 \pm 0.00 \mathrm{~mm}^{2}$, ctrl-Ab: $0.00 \pm 0.00 \mathrm{~mm}^{2}$, Figure $5 \mathrm{~B}, \mathrm{C}$ ) and Texas Red ${ }^{\circledast}$ cadaverine antibody staining (NMO-Ab: $0.04 \pm 0.07 \mathrm{~mm}^{2}$, ctrl-Ab: $0.00 \pm 0.00$ $\mathrm{mm}^{2}$, Figure $5 \mathrm{E}, \mathrm{F}$ ). This is contrary to the data we obtained from $\lg$ and fibrinogen measurements and confirms that deposits of both proteins can still be detected after the integrity of the BBB is re-established. By using the vascular tracers FITC-albumin and Texas Red $^{\circledR}$ cadaverine we could narrow down the time frame of increased BBB leakage to between 3 and $24 \mathrm{~h}$ after lesion induction. This also demonstrates that the BBB is closed to proteins with molecular sizes of $\geq 690$ Da before astrocytes repopulate the lesion (Figure 3). 
In addition, some extravasation of tracers was also observed $6 \mathrm{~h}$ after injection of ctrl-Ab and human complement, but represented only $48.8 \%$ (FITC-albumin) and 52.3\% (Texas Red ${ }^{\circledR}$ cadaverine) of the extent of extravasation detected after injection of NMO-Ab (Figure $5 \mathrm{C}, \mathrm{F}$ ).
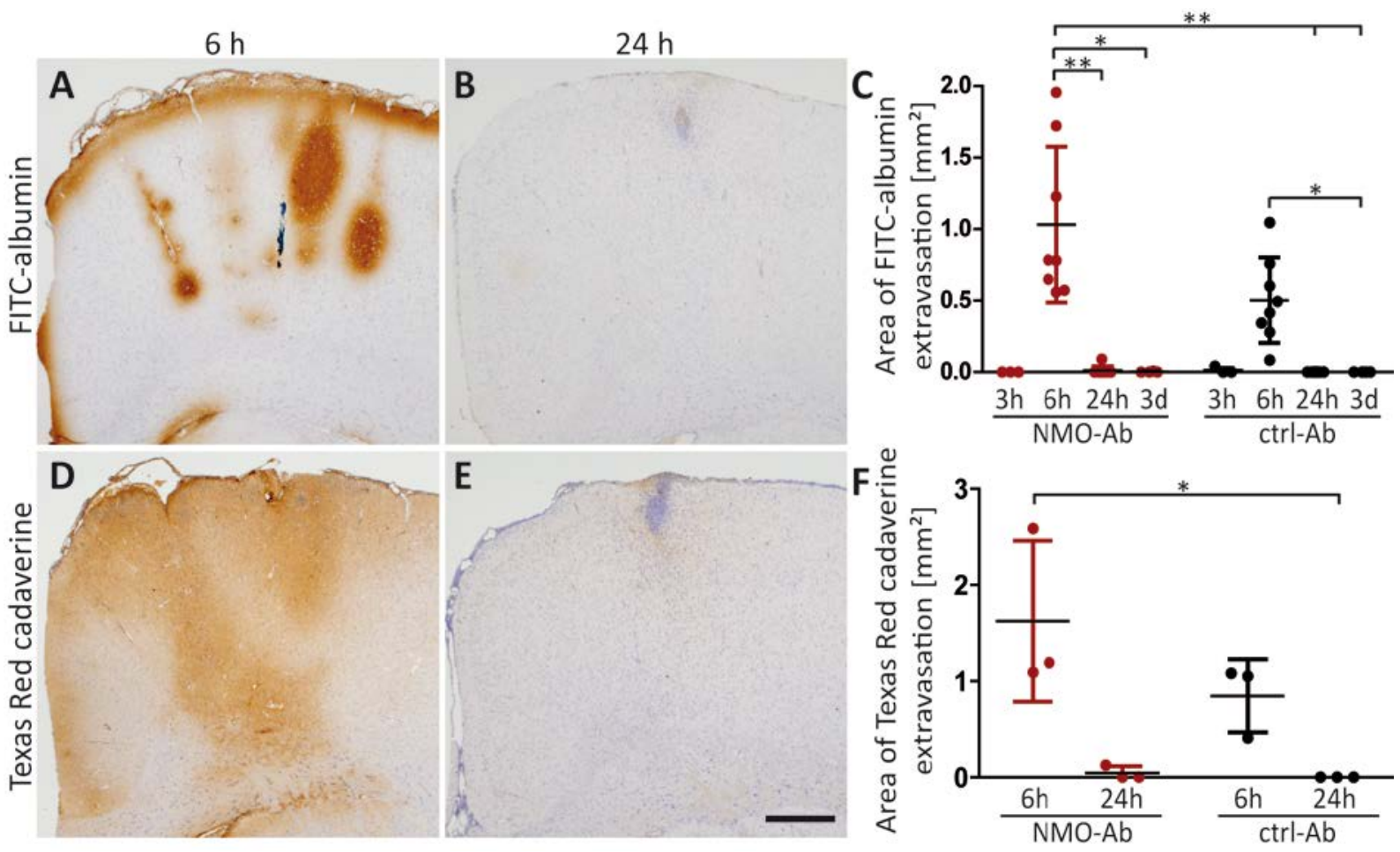

Figure 5: Restoration of the BBB within $24 \mathrm{~h}$ of focal intracerebral injection of NMO-Ab and human complement

$6 \mathrm{~h}$ after focal injection a prominent extravasation of FITC-albumin (A) and Texas Red ${ }^{\circledR}$ cadaverine (D) into the brain parenchyma was observed. No vascular leakage of either molecule was detected $24 \mathrm{~h}$ after stereotactic injection (B: FITC-albumin, E: Texas Red ${ }^{\circledR}$ cadaverine). Quantification of extravasation demonstrated that the BBB was permeable for FITC-albumin and Texas Red ${ }^{\circledR}$ cadaverine at $6 \mathrm{~h}$. However, it was restored within $24 \mathrm{~h}$ after focal injection of $A b$ and human complement. Moreover, no extravasation of FITC-albumin was observed at $3 \mathrm{~h}$ and $3 \mathrm{~d}$ after lesion induction. At $6 \mathrm{~h}$ after stereotactic injection, some extravasation of both tracer molecules was also detected in animals injected with ctrl-Ab and human complement. However, the area of extravasation was substantially smaller in ctrl-Ab injected animals and covered around $50 \%$ of the area observed in NMO-Ab injected animals (FITC-albumin: $3 \mathrm{~h}: n=3,6 \mathrm{~h}: n=8,24 \mathrm{~h}: n=9,3 \mathrm{~d}: n=4$, C, Texas Red ${ }^{\circledR}$ cadaverine: $n=3, \mathbf{F})$. Kruskal-Wallis test followed by Dunn's multiple comparison test ${ }^{*} p<0.05,{ }^{* *} p<0.01$. Graphs are shown as mean \pm SD. Scale bar $500 \mu \mathrm{m}$

\subsection{Co-culture of rat brain endothelial cells (RBEC) with astrocytes as an in- vitro model of the BBB did not result in increased TEER values}

$24 \mathrm{~h}$ after lesion induction the greatest extent of astrocyte loss was observed (Figure $3 \mathrm{G}$ ). However, no extravasation of i.v. injected exogenous tracer molecules was detected at that time point in vivo (Figure 5). Therefore, the direct influence of factors released by astrocytes on the transendothelial electrical resistance (TEER) of primary RBEC monolayers was investigated in vitro. TEER measurements are an important readout to assess the integrity of the RBEC monolayer. They represent the permeability of tight junctions (TJ) to sodium ions. 
Therefore, the higher the resistance, the lower the permeability of the EC monolayer to sodium ions.

$17 \mathrm{~d}$ old Wistar rats were used for the isolation of primary RBEC. Isolated RBEC grew in a dense monolayer and had a spindle shaped morphology. Additionally, immunocytochemistry demonstrated the localization of the endothelial cell marker von Willebrand factor (vWF) in the Weibel-Palade bodies of RBEC (Figure $6 \mathrm{~A}$ ). Furthermore, RBEC expressed the TJ molecules occludin, claudin-3 and -5 which were localized at the cell borders (Figure $6 \mathrm{~A}$ ). The expression of TJ molecules implicates a preservation of the primary BBB phenotype.

In order to investigate the influence of factors released by astrocytes on the TEER values of the RBEC monolayer, an indirect co-culture model was developed. A transwell system was used in which primary rat astrocytes were plated in the basolateral compartment and RBEC in the apical compartment (Figure 6B). Starting from day 3 after isolation TEER values were measured daily. The electrical resistance increased until day 6 after RBEC isolation. Starting from day 6 on, TEER values decreased. This was due to RBEC apoptosis and formation of holes in the monolayer as assessed by light microscopy. Co-culture of RBEC with astrocytes did not result in a significant change of electrical resistance compared to RBEC cultured alone at any time point investigated (Figure 6C).

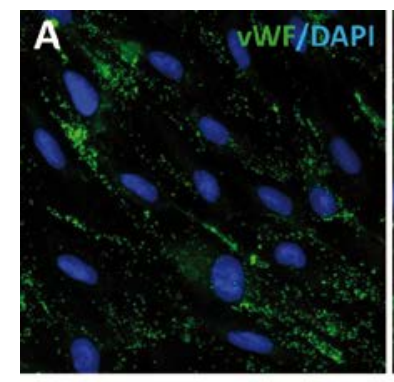

B

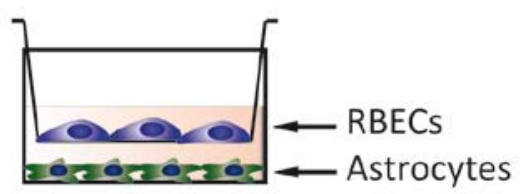

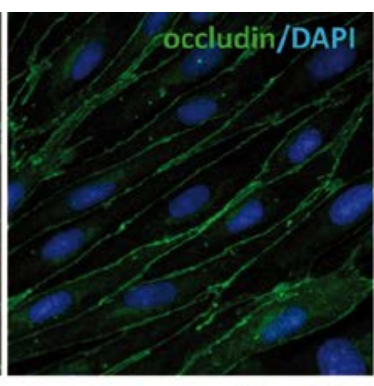
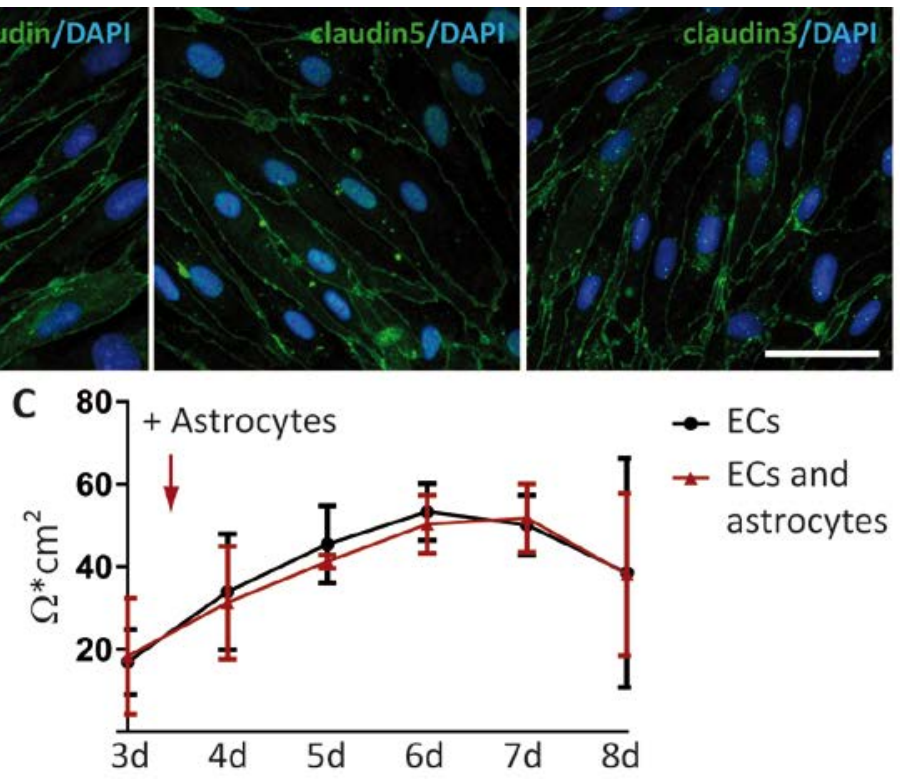

$\rightarrow$ ECS

- ECs and astrocytes

Figure 6: Co-culture of RBEC with astrocytes as an in vitro model of the BBB did not result in altered TEER values.

Immunocytochemistry of endothelial marker molecules is depicted in A. Isolated primary RBEC stain positively for the endothelial cell marker VWF, which is stored in the Weibel-Palade bodies. Additionally they display the TJ molecules occludin, CLDN3 and CLDN5 located at the endothelial cell-cell contacts (A). Co-culture model of the BBB with primary rat astrocytes growing in the basolateral compartment, and RBEC plated in the apical compartment (B). TEER values of the RBEC in the inserts were measured daily after day 3 . The first 
measurement was performed from RBEC grown alone. Subsequently, inserts containing RBEC were transferred into wells with a confluent astrocyte monolayer (indicated by red arrow). However, co-culture of astrocytes did not affect TEER values of RBEC (pooled data of 3 independent experiments performed in triplicates, C). Data are shown as mean \pm SD. Scale bar $40 \mu \mathrm{m}$.

\subsection{Platelet-derived growth factor receptor $B$ (PDGFRß) positive cells are in close proximity to brain vessels $6 \mathrm{~h}$ and $24 \mathrm{~h}$ after lesion induction}

It has been reported previously that pericytes play an important role for the development of the BBB and for its maintenance in the adult animal in vivo (Armulik et al., 2010; Bell et al., 2010; Daneman et al., 2010b). They are - beside EC, the basal lamina and astrocytes - a further component of the BBB. Pericytes ensheath blood vessels and share a basement membrane with EC. CNS vessels have the highest density of pericyte coverage in the body (Shepro and Morel, 1993). In order to assess the presence of pericytes within astrocyte depleted lesions animals were stereotactically injected with NMO-Ab and human complement and sacrificed $6 \mathrm{~h}$ and $24 \mathrm{~h}$ later. Perilesional brain tissue was used as control. PDGFRß, a marker for pericytes, was used to investigate the presence of pericytes at the BBB. In addition, to visualize the blood vessels, the basal lamina was stained using an Ab against laminin $\gamma 1$ (LAM 1$)$. Astrocyte depleted areas in NMO-Ab injected animals were visualized using anti-GFAP Ab.

In perilesional tissue PDGFRß positive cells (green) line the brain vessels which are also surrounded by a LAMY1 (red) positive basal lamina (Figure 7 A). After 6 h (Figure 7 B) and $24 \mathrm{~h}$ (Figure $7 \mathrm{C}$ ) of NMO lesion induction, expression of PDGFRß on LAMY1 positive vessels was not altered in astrocyte depleted areas compared to perilesional tissue. This indicates that pericytes were not markedly reduced by depletion of astrocytes in NMO-like lesions. 


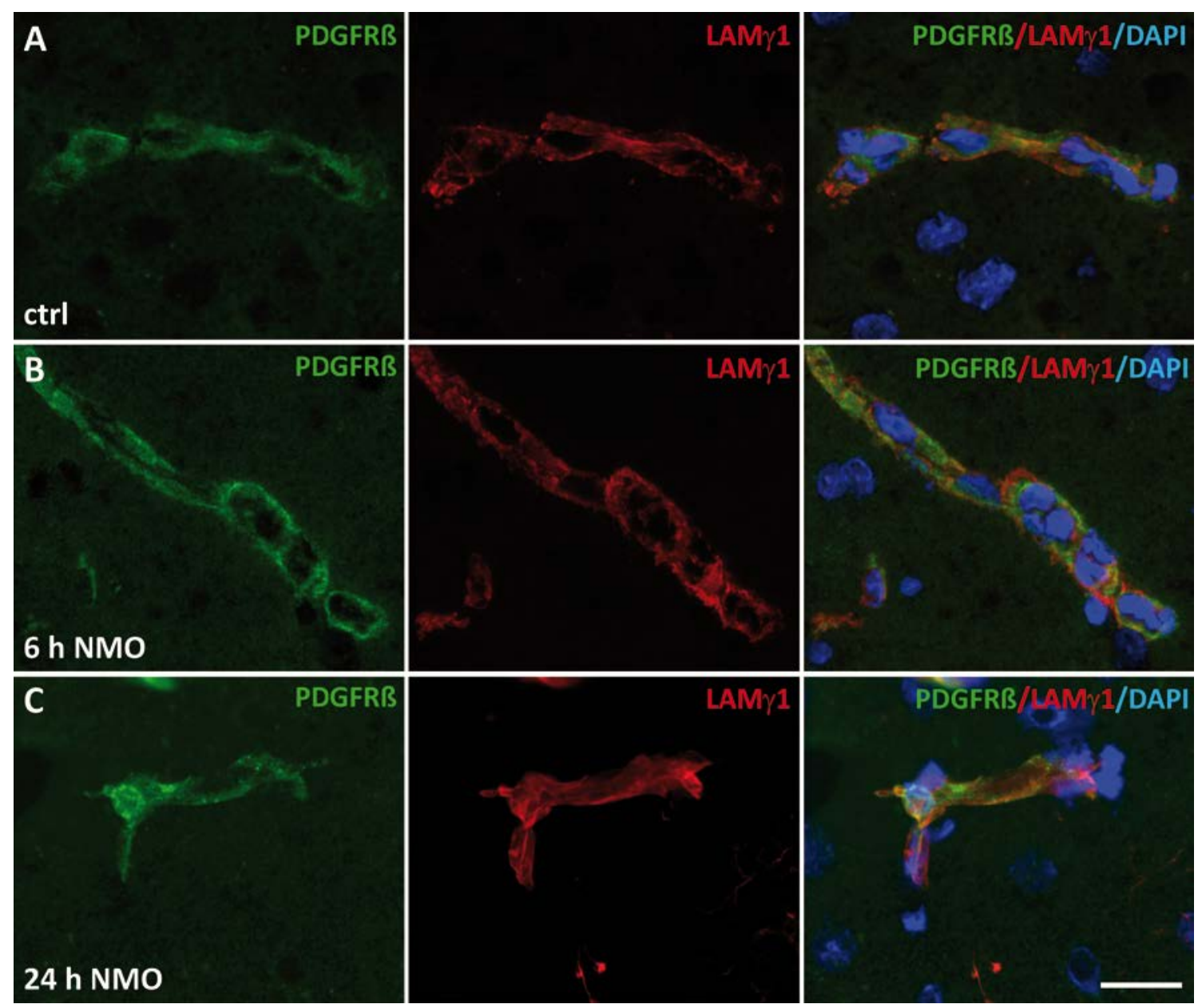

Figure 7: No loss of PDGFRß positive cells from the blood vessels in NMO-like lesions $6 h$ and $24 h$ after focal NMO-Ab injection

PDGFRß positive cells (green) ensheath brain vessels marked by the basal lamina component LAMY1 (red) in perilesional brain tissue (A). The staining pattern is not altered in astrocyte depleted lesions $6 \mathrm{~h}$ (B) and $24 \mathrm{~h}$ (C) after lesion induction. Scale bar $20 \mu \mathrm{m}$

\subsection{Characterization of tight junctions (TJ) in NMO-like lesions}

TJ are the major structure responsible for the low permeability of the BBB. They are located at the cell-cell contacts of adjacent EC and prevent the paracellular diffusion of blood-borne molecules into the CNS. Disruption of TJ morphology or loss of TJ proteins from the vasculature were implied in the loss of BBB integrity in neuroinflammatory diseases like multiple sclerosis (MS) or its model experimental autoimmune encephalomyelitis (EAE) (Argaw et al., 2009; Errede et al., 2012; Kirk et al., 2003; Plumb et al., 2002; Wolburg et al., 2003). In focal NMO-like lesions a transient loss of the BBB integrity is observed. In order to investigate a correlation between a loss of TJ proteins and the breakdown of the BBB, the transmembrane TJ proteins occludin, claudin-3 and -5 were investigated on the immunohistochemical and mRNA levels. In addition, electron microscopy (EM) of blood vessels in lesions was performed. 


\subsubsection{Transient loss of occludin from $\mathrm{TJ}$ after lesion induction}

The transmembrane TJ proteins occludin, claudin-3 and -5 are highly expressed in the brain vasculature and disruption of their expression has been implied in the dysfunction of the BBB (Kirk et al., 2003; Liebner et al., 2000a; Plumb et al., 2002; Wolburg et al., 2003). In order to investigate whether the transient breakdown of the BBB observed during the development of focal NMO-like lesions correlates with a loss of TJ molecules from the brain vasculature, a time course study was performed. Animals were injected with NMO- or ctrlAb together with human complement and sacrificed after $6 \mathrm{~h}, 10 \mathrm{~h}, 24 \mathrm{~h}, 3 \mathrm{~d}$ or $6 \mathrm{~d}$. Since a certain loss of BBB integrity was also observed in animals injected with ctrl-Ab and human complement (Figure 4 and Figure 5) uninjected brain tissue from naïve rats was used as additional control. Immunofluorescent staining with Ab against occludin, claudin-3 and -5 was used to investigate TJ proteins at the BBB. Double-labeling with the basal lamina component LAMY1 was performed to visualize blood vessels. Immunohistochemistry for GFAP demarcated the astrocyte lesion border in NMO-Ab injected animals. The percentage of LAMY1-positive blood vessels with vascular localization of the TJ proteins occludin, claudin-3 or -5 was determined for each animal. In NMO-Ab injected animals, blood vessels within areas of GFAP loss were evaluated, whereas in ctrl-Ab injected animals the corresponding area adjacent to the injection site, marked by Monastral blue, were assessed.

In uninjected, naïve controls, sharply defined strands of occludin immunoreactivity (green) marking the paracellular cleft of adjacent EC within the LAM 1 1-immunopositive basal membrane (red) were observed (Figure 8 A). Quantification of occludin positive vessels revealed occludin immunoreactivity in $93.3 \pm 3.3 \%$ of the vessels in the brain of uninjected controls. However, loss of occludin immunoreactivity from TJ in LAMY1 positive vessels was observed $10 \mathrm{~h}$ and $24 \mathrm{~h}$ after lesion induction (3.5 $\pm 2.2 \%(10 \mathrm{~h})$ and $3.4 \pm 3.4 \%(24 \mathrm{~h})$ of vessels are immunopositive for occludin, Figure $8 \mathrm{~A}, \mathrm{D}) .3 \mathrm{~d}$ after lesion induction a vascular localization of occludin within GFAP depleted areas was again observed in $68.5 \pm 4.6 \%$ of the blood vessels (Figure $8 \mathrm{~A}, \mathrm{D}$ ), indicating occludin restoration at TJ. $6 \mathrm{~d}$ later, the localization of occludin at the TJ was restored and no difference in occludin immunoreactivity was detected compared to uninjected controls. Here, $94.6 \pm 3.0 \%$ of the vessels stained positively for occludin which was similar to $93.3 \pm 3.3 \%$ in naïve controls (Figure $8 \mathrm{D}$ ). A less pronounced reduction of occludin positive vessels was also observed in ctrl-Ab injected animals $10 \mathrm{~h}$ and $24 \mathrm{~h}$ after stereotactic injection (38.2 $\pm 15.2 \%$ and $31.9 \pm 31.5 \%$ of vessels were positive for occludin at the respective time points) (Figure $8 D$ ). 
In contrast, no loss of claudin-3 and -5 immunoreactivity (green) from LAM 1 positive vessels (red) was observed at any time point investigated (Figure 8 B, C, E, F). In both, uninjected controls and GFAP depleted lesions $10 \mathrm{~h}$ after lesion induction, sharply defined claudin-3 and -5 immunoreactivity marking the paracellular cleft of adjacent EC within the LAMY1-immunopositive basal membrane was observed (Figure 8 B, C). Assessment of the percentage of claudin-3 and -5 positive vessels revealed no alterations of claudin- 3 and -5 immunoreactivity $6 \mathrm{~h}, 10 \mathrm{~h}, 24 \mathrm{~h}, 3 \mathrm{~d}$ or $6 \mathrm{~d}$ after lesion induction compared to ctrl-Ab injected animals and uninjected controls (Figure $8 \mathrm{E}, \mathrm{F}$ ). Claudin-3 immunoreactivity was detected in $76.9 \% \pm 8.7$ ( $24 \mathrm{~h}$ NMO-Ab) to $91.9 \% \pm 2.7$ ( $3 \mathrm{~d}$ ctrl-Ab) of LAMp1 positive vessels (Figure $8 \mathrm{E}$ ), and claudin-5 immunoreactivity was detected in $85.5 \% \pm 8.1$ (10 h NMO-Ab) to $97.1 \% \pm 1.9$ ( $6 \mathrm{~d}$ NMO-Ab) of LAMp1 positive vessels (Figure $8 \mathrm{~F}$ ).

In summary, a transient loss of occludin starting from $6 \mathrm{~h}$ after lesion induction was observed. Localization of occludin at the TJ was partially recovered $3 \mathrm{~d}$ after lesion induction and back to control levels after $6 \mathrm{~d}$. No loss of claudin-3 or -5 from the TJ was detected at any time point investigated. 

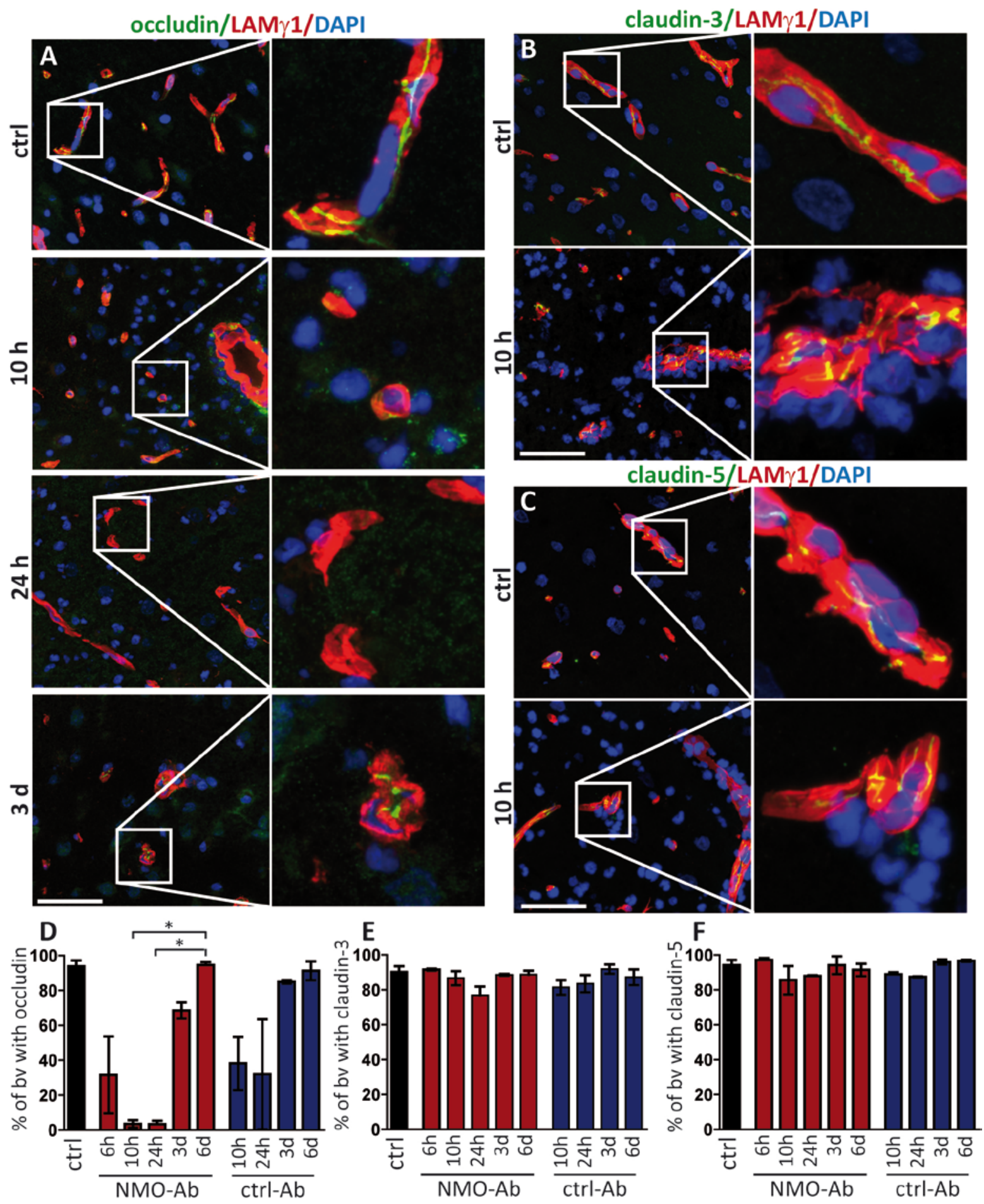

Figure 8: Loss of occludin from the blood vessels was observed in focal NMO-like lesions

Immunolabelling for occludin, claudin-3 and -5 (green) was performed to assess the localization of these TJ proteins in brain vessels (visualized with LAMY1, a marker of the basal lamina, red) around the injection site. Immunoreactivity for occludin (A), claudin-3 (B) and -5 (C) was localized at the TJ between adjacent EC which formed sharply defined, continuous strands in uninjected, naïve controls. However, loss of occludin immunoreactivity was observed $10 \mathrm{~h}$ and $24 \mathrm{~h}$ after lesion induction in astrocyte depleted areas. Vascular occludin localization was recovered to $68.5 \pm 4.6 \%$ within $3 \mathrm{~d}$ after focal injection of NMO-Ab and human complement (A). Quantification of occludin positive vessels confirms the transient loss of occludin immunoreactivity from the blood vessels in areas of GFAP loss. A less pronounced decrease of occludinimmunopositive vessels was also observed in animals injected with ctrl-Ab together with human complement $10 \mathrm{~h}$ and $24 \mathrm{~h}$ after focal injection (41-307 vessels/animal evaluated depending on astrocyte lesion size; ctrl 
$\mathrm{n}=3$, NMO-Ab/ctrl-Ab; 6h: $n=4 / 0 ; 10 \mathrm{~h}: n=3 / 3 ; 24 \mathrm{~h}: n=3 / 2 ; 3 \mathrm{~d}: n=3 / 3 ; 6 \mathrm{~d}: n=3 / 3$, D). In contrast, no loss of claudin-3 and -5 immunoreactivity was detected after lesion induction and sharply defined immunoreactivity for claudin-3 (B) and -5 (C) was observed at the TJ of LAMY1 positive vessels at $10 \mathrm{~h}$. Quantification of claudin-3 positive vessels (45-218 vessels/animal evaluated; $c t r l \mathrm{n}=3, \mathrm{NMO}-\mathrm{Ab} / \mathrm{ctrl}-\mathrm{Ab} ; 6 \mathrm{~h}: n=4 / 0 ; 10 \mathrm{~h}: n=2 / 3 ; 24 \mathrm{~h}$ : $n=3 / 2 ; 3 \mathrm{~d}: n=2 / 2 ; 6 \mathrm{~d}: n=3 / 3, \mathrm{E})$ and claudin-5 positive vessels (28-209 vessels/animal, ctrl $\mathrm{n}=3, \mathrm{NMO}-A b / c t r l-A b$; 6h: $n=4 / 0$; 10h: $n=3 / 3$; 24h: $n=3 / 2 ; 3 \mathrm{~d}: n=3 / 2 ; 6 \mathrm{~d}: n=3 / 3$, F) confirms this observation. Kruskal-Wallis test followed by Dunn's multiple comparison test $*_{p}<0.05$. Data are shown as mean \pm SD. Scale bar $50 \mu \mathrm{m}$. $b v=$ blood vessels.

\subsubsection{No morphological alterations of $\mathrm{TJ}$ on the ultrastructural level}

A transient loss of occludin immunoreactivity from the TJ of LAMY1 positive vessels was observed starting $6 \mathrm{~h}$ after lesion induction. Only about 3.5\% of the blood vessels showed occludin immunoreactivity $10 \mathrm{~h}$ and $24 \mathrm{~h}$ after lesion induction. However, no loss of claudin3 and -5 immunoreactivity was detected (Figure 8). In order to investigate whether the selective loss of occludin immunoreactivity from the TJ of brain vessels results in morphological alterations on the ultrastructural level, i.e. separation of adjacent EC, EM was performed. Animals were stereotactically injected with NMO-Ab and human complement and sacrificed $6 \mathrm{~h}$ and $24 \mathrm{~h}$ after lesion induction.

To ensure the presence of a fully developed astrocyte depleted lesion, the caudal part of the lesion was used for immunohistochemistry, while the rostral part was prepared for EM. GFAP-immunohistochemistry demonstrated the depletion of astrocytes. Using EM a close association between adjacent EC was observed in uninjected controls. No overt morphological TJ abnormalities or any separation of adjacent EC were detected $6 \mathrm{~h}$ and $24 \mathrm{~h}$ after lesion induction (Figure 9).
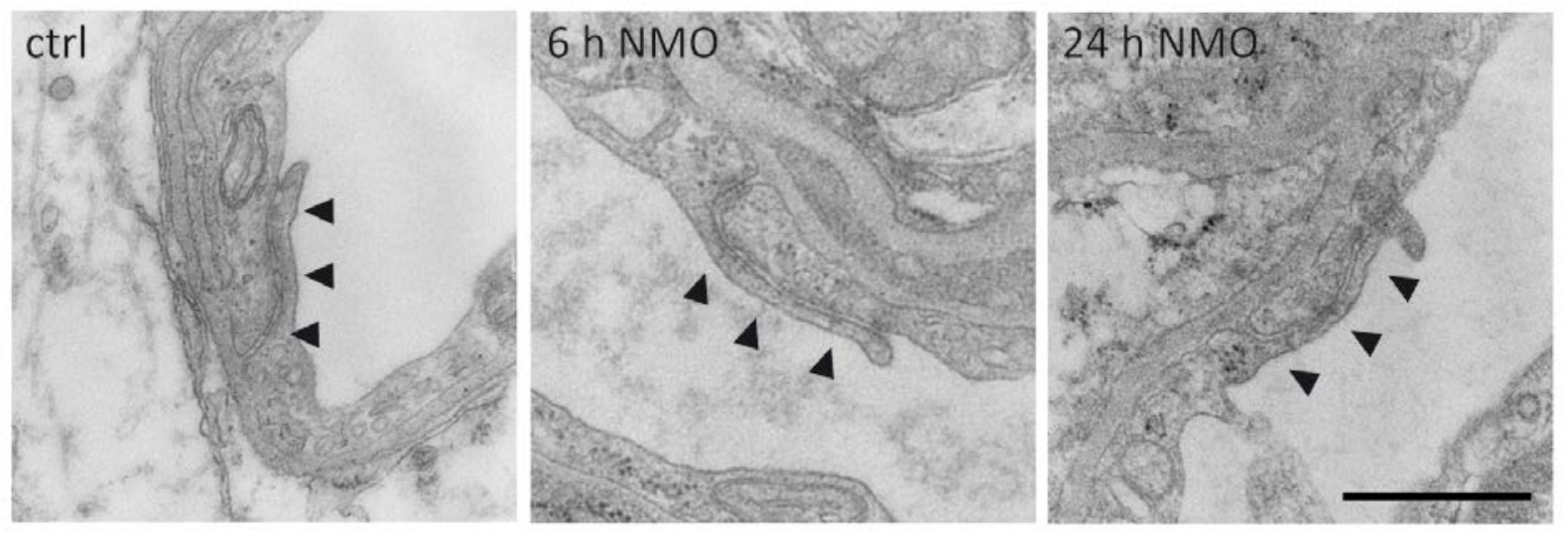

\section{Figure 9: Ultrastructure of $\mathrm{TJ}$ in cerebral blood vessels after lesion induction}

TJ were observed between adjacent EC in ultra-thin sections using EM. Injection of NMO-Ab and human complement did not result in overt morphological alterations of TJ complexes. Depicted images show representative TJ of brain blood vessels in uninjected controls and $6 \mathrm{~h}$ or $24 \mathrm{~h}$ after lesion induction. Arrows indicate endothelial cell-cell junctions. Scale bar $0.5 \mu \mathrm{m}$. 


\subsection{3 mRNA levels of occludin and claudin 5 are increased in NMO-like lesions}

In order to evaluate if changes in mRNA expression of TJ genes are induced during the development of NMO-like lesions a quantitative PCR study was performed. Animals were stereotactically injected with NMO- or ctrl-Ab together with human complement and perfused $10 \mathrm{~h}, 24 \mathrm{~h}, 3 \mathrm{~d}$ or $6 \mathrm{~d}$ after stereotactic injection. Uninjected brain tissue served as negative control. Subsequently, the tissue was cut into $10 \mu \mathrm{m}$ thick sections using a cryostat, mounted on polyethylene naphthalate membranes and stained for GFAP using a rapid staining protocol to avoid RNA degradation. Brain areas with loss of astrocytes were isolated under the microscope from NMO-Ab injected animals using laser capture microdissection. In animals injected with ctrl-Ab and human complement the area around the injection site was dissected or the respective brain area in uninjected controls. Subsequently, TaqMan Gene Expression Assay for occludin, claudin-3 and -5 were performed. The house keeping gene gapdh served as control. $C_{T}$ values for expression of $T J$ genes were normalized to $C_{T}$ values of gapdh to calculate $\Delta \mathrm{C}_{\mathrm{T}}$ values ( $\mathrm{C}_{\mathrm{T}} \mathrm{TJ}$ gene - $\mathrm{C}_{\mathrm{T}}$ gapdh). $\Delta \Delta \mathrm{C}_{\mathrm{T}}$ values were calculated by subtraction of the $\Delta C_{T}$ value of uninjected controls from $\Delta C_{T}$ values obtained after lesion induction.

$10 \mathrm{~h}$ and $24 \mathrm{~h}$ after stereotactic injection of NMO- or ctrl-Ab together with human complement, mRNA expression of occludin and claudin-5 were increased compared to uninjected controls $(\triangle \Delta C T$ values of NMO-/ctrl-Ab injected animals after $10 \mathrm{~h}$ : occludin=3.2/3.7, claudin-5=2.8/3.9; after $24 \mathrm{~h}$ : occludin=2.6/2.2, claudin-5=1.5/0.7). $3 \mathrm{~d}$ after stereotactic injection mRNA expression of occludin was similar to control levels while expression of claudin-5 was decreased $(\Delta \Delta C T$ values of NMO-/ctrl-Ab injected animals: occludin=-0.3/ 0.2, claudin-5=-1.7/-1.0). After $6 \mathrm{~d} \Delta \Delta C T$ values of $-0.7 /-0.4$ (occludin) and 0.1/-0.5 (claudin-5) were measured (Figure 10). No time dependent regulation of claudin-3 mRNA expression was observed, however an increased mRNA expression was measured $24 \mathrm{~h}$ after focal injection of NMO-Ab and human complement $(\Delta \Delta C T=2.6$, Figure 10). In summary, occludin and claudin-5 mRNA expression was increased $10 \mathrm{~h}$ and $24 \mathrm{~h}$ after stereotactic injection. No difference in mRNA expression was observed between animals injected with NMO- or ctrl-Ab together with human complement at the time points investigated. 


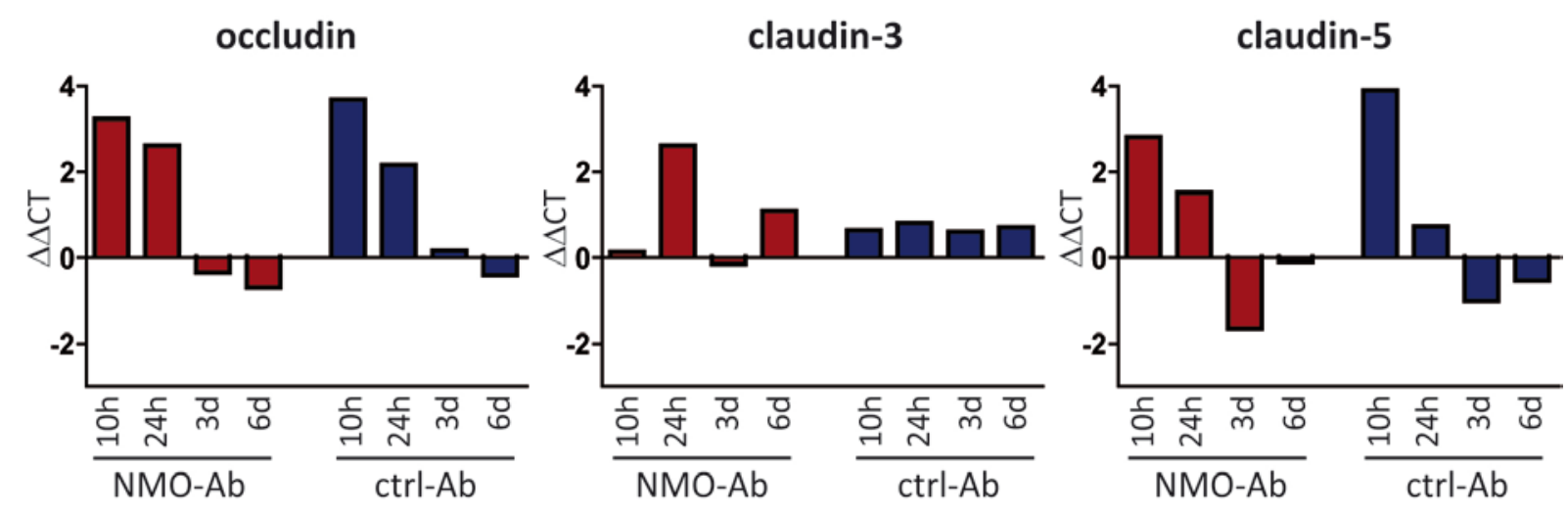

Figure 10: mRNA expression of occludin and claudin-5 were increased $10 \mathrm{~h}$ and $24 \mathrm{~h}$ after stereotactic injection

Increased expression of occludin and claudin-5 mRNA was measured $10 \mathrm{~h}$ and $24 \mathrm{~h}$ after stereotactic injection of NMO- or ctrl-Ab compared to uninjected controls. After $3 \mathrm{~d}$ and $6 \mathrm{~d}$ mRNA expression of occludin and claudin-5 were decreased (claudin-5 after $3 \mathrm{~d}$ ) or similar to uninjected controls. No time dependent regulation of claudin-3 was observed (number of animals measured at $10 \mathrm{~h} / 24 \mathrm{~h} / 3 \mathrm{~d} / 6 \mathrm{~d}$, occludin NMO: $n=2 / 3 / 2 / 4$, occludin ctrl: $n=3 / 4 / 3 / 4$, claudin-3 NMO: $n=3 / 4 / 2 / 4$, claudin-3-ctrl: $n=4 / 3 / 3 / 4$, claudin-5 NMO: $n=2 / 3 / 2 / 4$, claudin-5 ctrl: $n=4 / 2 / 3 / 4)$.

\subsection{Infiltration of polymorphonuclear cells (PMN) correlates with FITC- albumin extravasation $6 \mathrm{~h}$ after lesion induction}

The infiltration of immune cells into the brain parenchyma has been implied to mediate the breakdown of the BBB in diseases like NMO, multiple sclerosis or cerebral ischemia and their animal models (Bartholomaus et al., 2009; Carlson et al., 2008; Grossman et al., 1986; Morrissey et al., 1996; O'Riordan et al., 1996; Virapongse et al., 1986). Moreover, infiltration of immune cells into the brain has been described upon induction of focal NMO-like lesions $24 \mathrm{~h}$ and $7 \mathrm{~d}$ after lesion induction (Wrzos et al., 2014). In order to investigate the sequence of infiltrating immune cells in NMO-like lesions, animals were stereotactically injected with NMO-Ab and human complement and subsequently sacrificed after $3 \mathrm{~h}, 6 \mathrm{~h}, 12 \mathrm{~h}, 24 \mathrm{~h}, 3 \mathrm{~d}$ or $7 \mathrm{~d}$. To visualize immune cell subsets immunohistochemistry against ED1 (macrophages/ activated microglia) and CD3 (T cells) was performed. In addition, chloroacetate esterase enzyme histochemistry (CAE) was used to label PMN.

To quantify infiltrating immune cell subsets, cells were enumerated using a light microscope with an ocular morphometric grid, and their density was calculated. $3 \mathrm{~h}$ after lesion induction no infiltration of immune cells was observed at the injection site (Figure $11 \mathrm{~B}$ ). However, after $6 \mathrm{~h}$ infiltration of PMN with a density of $223 \pm 101 \mathrm{PMN} / \mathrm{mm}^{2}$ was detected which increased to $842 \pm 71$ after $12 \mathrm{~h}$, and $801 \pm 308 \mathrm{PMN} / \mathrm{mm}^{2}$ after $24 \mathrm{~h}$. Subsequently the PMN density again decreased to $314 \pm 202 \mathrm{PMN} / \mathrm{mm}^{2} 3 \mathrm{~d}$ after lesion induction. $7 \mathrm{~d}$ after focal injection only scattered PMN were detected (2 $\pm 2 \mathrm{PMN} / \mathrm{mm}^{2}$ Figure $\left.11 \mathrm{~A}, \mathrm{~B}\right)$. 
Investigating the numbers of ED1 positive macrophages/activated microglia, only scattered ED1 positive cells were observed $6 \mathrm{~h}$ after lesion induction ( $2 \pm 2$ ED1 positive cells $/ \mathrm{mm}^{2}$ ) which subsequently increased until the density peaked $24 \mathrm{~h}$ after lesion induction with $453 \pm 72$ ED1 positive cells/mm². Numbers of macrophages/activated microglia subsequently decreased to $204 \pm 34$ ED1 positive cells $/ \mathrm{mm}^{2}\left(3 \mathrm{~d}\right.$ ) and to $72 \pm 57 \mathrm{ED} 1$ positive cells $/ \mathrm{mm}^{2}$ (7 d after lesion induction, Figure $11 \mathrm{~A}, \mathrm{~B})$. T cell numbers during the course of NMO-like lesion development remained low with a maximal density of $18 \pm 16$ CD3 positive T cells $/ \mathrm{mm}^{2} 7 \mathrm{~d}$ after lesion induction (Figure $11 \mathrm{~A}, \mathrm{~B}$ ).

The density of PMN $/ \mathrm{mm}^{2}$ was significantly higher compared to ED1 positive cells $(* p<0.05)$ and CD3 positive T cells $(* * * p<0.05) 6 \mathrm{~h}$ after focal injection of NMO-Ab and human complement (Figure $11 \mathrm{C}$ ). Therefore, a possible relationship between PMN and BBB permeability $6 \mathrm{~h}$ after lesion induction was investigated. The total number of parenchymal PMN around the injection site was determined and correlated with the measured area of FITC-albumin extravasation in serial sections. The number of PMN in the lesion correlated with the area of FITC-albumin extravasation $(* p=0.0291$, Pearson $r=0.6271)$ potentially suggesting a role of PMN in the regulation of BBB permeability during the initiation of NMO like lesions. 
A

A $\quad 6 \mathrm{~h}$

$6 \mathrm{~h}$

$3 d$

$7 d$
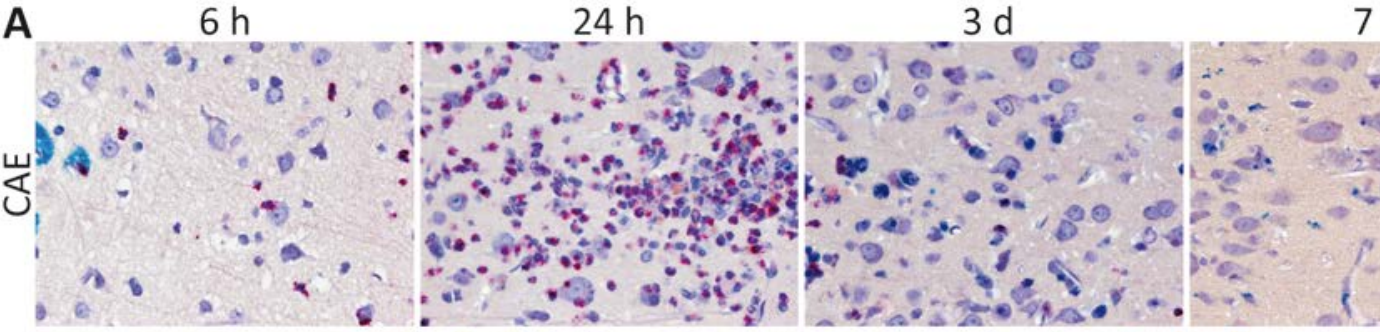

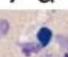
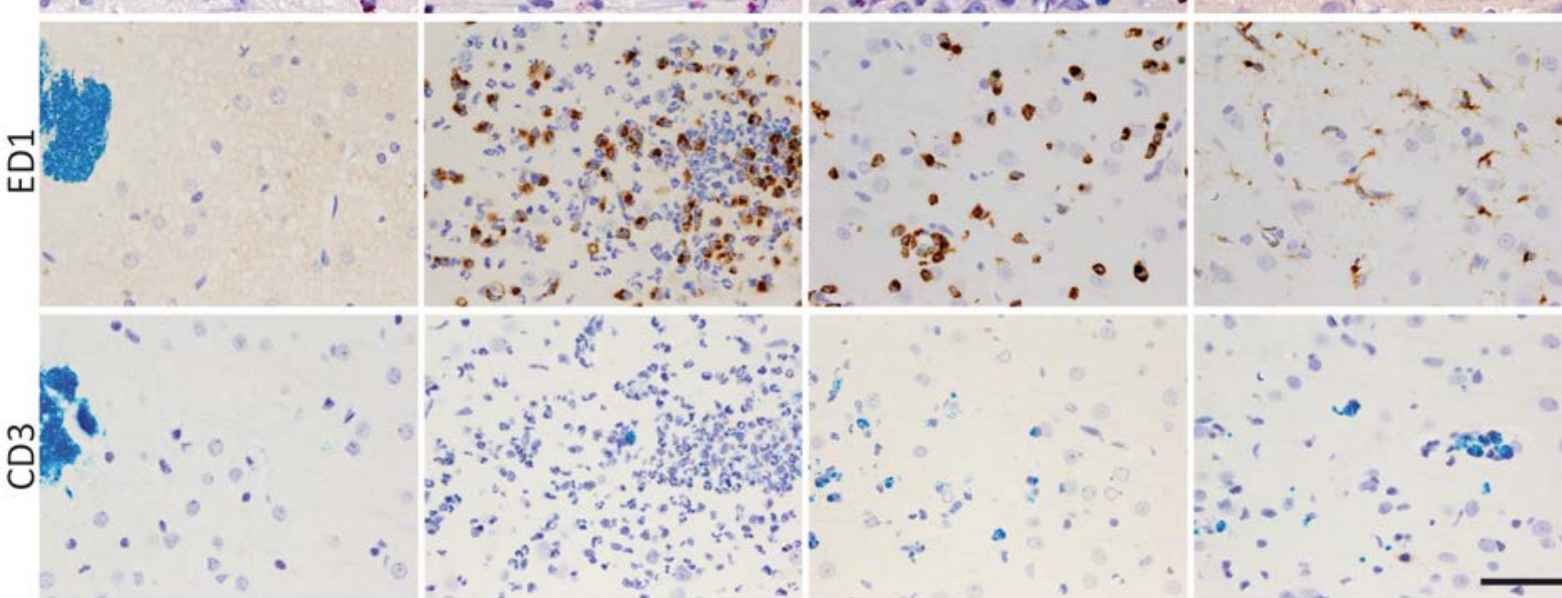

B

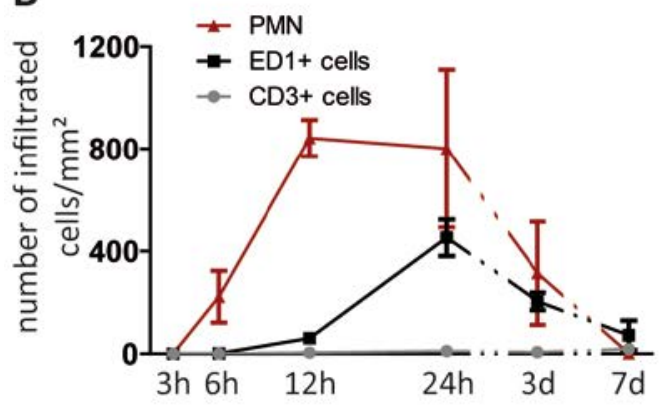

C

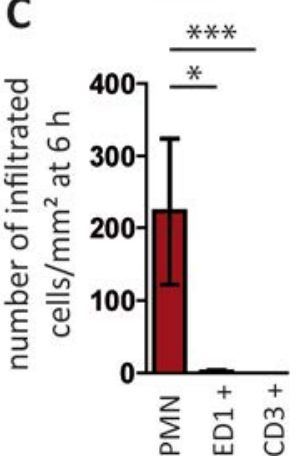

D

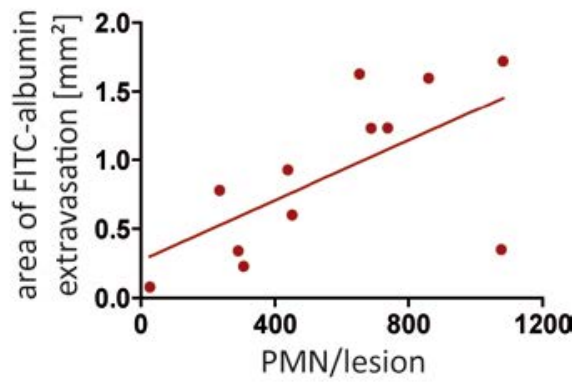

Figure 11: Strong infiltration of PMN correlated with extravasation of FITC-albumin $6 \mathrm{~h}$ after lesion induction

Representative photographs depicting the infiltration of PMN (CAE, bright pink), macrophages/activated microglia (ED1, brown) and T cells (CD3, brown) $6 \mathrm{~h}, 24 \mathrm{~h}, 3 \mathrm{~d}$ and $7 \mathrm{~d}$ after lesion induction (A). Quantification of the infiltration of infiltrated immune cell subsets: no immune cell infiltration was observed in the brain parenchyma $3 \mathrm{~h}$ after lesion induction. $6 \mathrm{~h}$ after lesion induction infiltration of PMN was observed. PMN numbers increased with a maximal density between $12 \mathrm{~h}$ and $24 \mathrm{~h}$ after lesion induction and subsequently decreased. Infiltration of ED1 positive cells was observed starting at $6 \mathrm{~h}$ after lesion induction until they reached the highest density at $24 \mathrm{~h}$ after lesion induction. Subsequently the number of ED1 positive macrophages/activated microglia decreased. Only few $\mathrm{CD} 3$ positive T cells were detected (number of animals analyzed in CAE/ED1/CD3: $3 \mathrm{~h} n=4 / 4 / 4,6 \mathrm{~h}: n=8 / 7 / 7,12 \mathrm{~h} n=2 / 2 / 2,24 \mathrm{~h} n=7 / 6 / 7,3 \mathrm{~d}: n=6 / 6 / 6,7 \mathrm{~d} 6 / 6 / 5$, B). PMN represented the highest density of all infiltrated immune cell subsets $6 \mathrm{~h}$ after lesion induction $(n=8 / 7 / 7$, C). The number of infiltrated PMN at this time point correlated with the area of FITC-albumin extravasation into the brain parenchyma (Pearson correlation for normally distributed samples ${ }^{*} p=0.0291$, Pearson $r=0.6271$, $n=12$, D). Monastral blue marks the injection site. Kruskal-Wallis test followed by Dunn's multiple comparison test $* p<0.05, * * * p<0.001$. Data are shown as mean \pm SD. Scale bar $50 \mu \mathrm{m}$. 


\subsection{Effect of PMN depletion on lesion development}

\subsubsection{Depletion of PMN prevents astrocyte depletion, breakdown of the BBB and occludin loss $6 \mathrm{~h}$ after lesion induction}

The number of PMN in the lesion correlated with the area of FITC-albumin extravasation $6 \mathrm{~h}$ after lesion induction. To investigate whether PMN infiltration contributes to the breakdown of the BBB, PMN depletion studies were performed. In order to deplete PMN from the blood, animals were treated with $1 \mathrm{ml} / \mathrm{kg}$ body weight anti-PMN antiserum (AIA51140; Accurate Chemical and Scientific Corporation), at $18 \mathrm{~h}$ prior and directly after stereotactic injection of NMO- or ctrl-Ab together with human complement. Control rats received normal serum (normal rabbit serum; AIS403, Accurate Chemical and Scientific Corporation). To investigate the BBB integrity, FITC-albumin was injected $40 \mathrm{~min}$ before perfusion. Animals were sacrificed $6 \mathrm{~h}$ after lesion induction (Figure $12 \mathrm{~A}$ ). CAE enzyme histochemistry was performed to visualize PMN. Immunohistochemistry for (i) FITC-albumin, (ii) astrocytes (GFAP), (ii) TJ proteins (occludin, claudin-3 and -5 ) and the basal lamina (LAMY1) were performed to investigate (i) BBB integrity, (ii) astrocyte depletion and (iii) loss of TJ proteins from the vasculature after PMN depletion.

To verify the depletion of PMN blood samples were collected before treatment and $6 \mathrm{~h}$ after lesion induction. Blood smear samples were analyzed by Giemsa stain. The number of PMN was counted among at least 300 cells on each slide and the number of PMN/100 white blood cells (WBCs) was determined. In blood samples obtained before treatment, $9.5 \pm 3.0$ PMN/100 WBCs were detected. After treatment with normal serum $14.7 \pm 5.2 \mathrm{PMN} / 100$ WBCs were observed in the blood samples. Anti-PMN antiserum treated animals, displayed a decrease of PMN numbers by $94 \%$ to $0.9 \pm 0.5$ PMN/100 WBCs. PMN numbers in normal serum treated rats were significantly higher compared to PMN numbers after treatment with anti-PMN serum $\left({ }^{* * *} p<0.001\right)$. Depletion of PMN was also confirmed in the brain parenchyma using CAE enzyme histochemistry. In rats treated with normal, but not antiPMN antiserum, infiltrated PMN were detected at the injection site after intracerebral injection of NMO-Ab and human complement (normal serum: $328 \pm 136 \mathrm{PMN} / \mathrm{mm}^{2}$, antiPMN serum: $0 \pm 0 \mathrm{PMN} / \mathrm{mm}^{2}, * * * p<0.001$, Figure $\left.12 \mathrm{~B}, \mathrm{C}\right)$. Some infiltration of PMN was also observed after stereotactic injection of ctrl-Ab and human complement in rats treated with non-immune serum (129 $\left.\pm 78 \mathrm{PMN} / \mathrm{mm}^{2}\right)$, reaching $39.3 \%$ of the $\mathrm{PMN}$ numbers observed in NMO-Ab injected animals (Figure $12 \mathrm{~B}, \mathrm{C}$ ). 
Furthermore the permeability of the BBB after cerebral injection of ctrl-Ab/NMO-Ab together with human complement was assessed in PMN depleted and non-depleted animals. In non-PMN-depleted animals, a prominent extravasation of the exogenous tracer molecule FITC-albumin into the brain parenchyma was observed $6 \mathrm{~h}$ after lesion induction. A substantially smaller area of FITC-albumin extravasation was also detected after stereotactic injection of ctrl-Ab and human complement (area of FITC-albumin extravasation in $\mathrm{mm}^{2}$ : NMO-A $b=0.9 \pm 0.5$; ctrl-Ab=0.5 $\pm 0.3,58.8 \%$ Figure $12 \mathrm{E}$ ). Depletion of PMN markedly reduced the extravasation of FITC-albumin in both NMO- and ctrl-Ab injected animals (area of FITC-albumin extravasation in $\mathrm{mm}^{2}$ : NMO-Ab=0.06 \pm 0.06 ; ctrl-Ab=0.05 \pm 0.1 ), which was significant compared to non-PMN depleted NMO-Ab injected animals $\left({ }^{*} p<0.01\right.$, Figure 12 $D, E)$.

In addition, depletion of astrocytes was assessed by GFAP immunohistochemistry. $6 \mathrm{~h}$ after focal injection of NMO-Ab and human complement, well demarcated GFAP depleted lesions were observed (area of GFAP-loss $0.6 \pm 0.4 \mathrm{~mm}^{2}$ ), whereas in ctrl-Ab injected animals no astrocyte loss was detected (area of GFAP-loss $0.0 \pm 0.0 \mathrm{~mm}^{2},{ }^{*} p<0.05$ ). Surprisingly, depletion of PMN in rats completely inhibited astrocyte lesion formation $6 \mathrm{~h}$ after focal injection of NMO-Ab and human complement (area of GFAP-loss $0.0 \pm 0.0 \mathrm{~mm}^{2}, * * * \mathrm{p}<0.001$, Figure $12 \mathrm{~F}, \mathrm{G})$.

Furthermore, the effect of PMN depletion on the localization of occludin, claudin- 3 and -5 at the TJ in LAMY1 positive blood vessels was investigated $6 \mathrm{~h}$ after intracerebral injection of NMO-Ab and human complement. The percentage of occludin positive vessel in uninjected brain tissue was $93.9 \pm 3.3 \%$. A significant reduction to $30.6 \pm 20.2 \%$ occludin positive vessels was observed $6 \mathrm{~h}$ after lesion induction $(* * \mathrm{p}<0.01)$. However, this reduction was not observed in PMN depleted animals ( $87.1 \pm 1.9 \%$ occludin positive vessels). The numbers of claudin-3 and -5 positive vessels were not altered $6 \mathrm{~h}$ after NMO-Ab injection with and without PMN depletion compared to untreated controls. The percentages of LAMY1 positive vessels with claudin-3 immunoreactivity were: uninjected brain tissue=90.4 $\pm 3.1 \%$; NMO$A b=91.5 \pm 0.5 \%$; and NMO-Ab after PMN depletion=91.1 $\pm 2.6 \%$. The percentages of LAM 1 positive vessels with claudin-5 immunoreactivity were: uninjected brain tissue=94.2 $\pm 2.9 \%$; $\mathrm{NMO}-\mathrm{Ab}=91.5 \pm 3.5 \%$; and NMO-Ab after PMN depletion=92.1 $\pm 2.7 \%$. (Figure $12 \mathrm{H}$ ).

These data indicate a crucial role for PMN in the development of NMO-like lesions, since depletion of PMN prevents breakdown of the BBB, loss of occludin from TJ and loss of astrocytes. In addition I showed in the presented thesis that focal injection of ctrl-Ab and 
human complement alone resulted in the infiltration of PMN (Figure 12) a subsequent breakdown of the BBB (Figure 4, Figure 5 and Figure 12) and loss of occludin from the TJ

(Figure 8), however substantially milder than in NMO-Ab injected rats. This emphasizes the importance of PMN in the regulation of BBB permeability.

Figure 12: Depletion of PMN inhibited astrocyte lesion formation, breakdown of the BBB and loss of occludin form the TJ $6 \mathrm{~h}$ after lesion induction

Experimental setup to assess the influence of PMN in the development of focal NMO-like lesions is depicted in (A). Focal injection of NMO-Ab or ctrl-Ab together with human complement results in the infiltration of CAE positive PMN (pink). Animals treated with normal rabbit serum showed 2.5 times more infiltrated PMN at the $\mathrm{NMO}-\mathrm{Ab}$ injected hemisphere than the ctrl-Ab injected hemisphere. Depletion of PMN from the blood is reflected by the absence of PMN infiltration in the cortex $6 \mathrm{~h}$ after focal injection of NMO- or ctrl-Ab together with human complement (B, quantification: $\mathbf{C}$ ). Furthermore, depletion of PMN decreased the permeability of the BBB to FITC-albumin $6 \mathrm{~h}$ after focal injection of NMO- or ctrl-Ab together with human complement (D, quantification: E). Loss of GFAP immunoreactivity was observed $6 \mathrm{~h}$ after focal injection of NMO-Ab, but not ctrl-Ab together with human complement. Loss of GFAP positive cells was inhibited by treatment with antiPMN serum (F, quantification: $\mathbf{G}$ ). C, E, G: $n=6$, pooled data of 2 independent experiments. Furthermore, the localization of occludin, claudin-3 and-5 at the TJ was investigated in PMN depleted and non-depleted animals $6 \mathrm{~h}$ after NMO-Ab injection. Injection of NMO-Ab together with human complement resulted in selective loss of occludin immunoreactivity from the TJ in normal serum injected animals. PMN-depletion inhibited loss of occludin immunoreactivity from blood vessels and was not altered compared to uninjected control brain. No changes in the percentage of claudin- 3 and -5 positive blood vessels were observed $6 \mathrm{~h}$ after lesion induction in PMN-depleted or non-depleted animals compared to uninjected control brain (ctrl $n=3,6 \mathrm{~h}$ NMO-Ab anti-PMN and ctrl: $n=4$, H). Kruskal-Wallis test followed by Dunn's multiple comparison test ${ }^{*} p<0.05,{ }^{* *} p<0.01$, ${ }^{* * *} p<0.001$. Data are shown as mean \pm SD. F: Dotted lines indicate area of GFAP loss. Scale bars: $50 \mu \mathrm{m}$ (B) and $500 \mu \mathrm{m}(\mathbf{D}, \mathbf{F})$ 

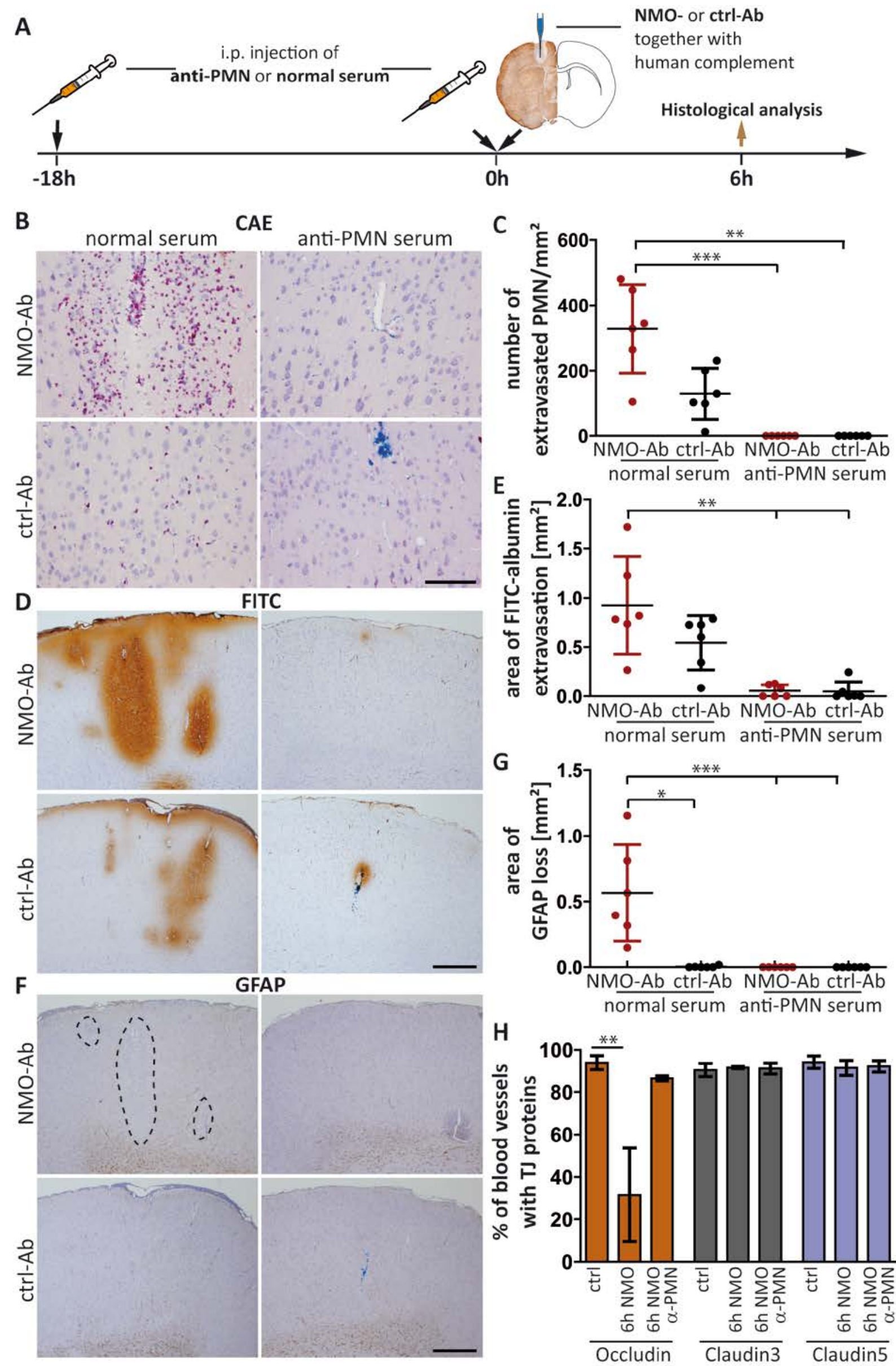


\subsubsection{Treatment with anti-PMN antiserum decreases immune cell numbers in the blood}

In order to investigate if anti-PMN antiserum selectively depletes PMN without affecting the numbers of other immune cell subsets flow cytometry was performed. Rats were treated with either anti-PMN antiserum (AIA51140; Accurate Chemical and Scientific Corporation) or normal serum (normal rabbit serum; AIS403, Accurate Chemical and Scientific Corporation) as described above (section 3.6.1). Blood was collected $6 \mathrm{~h}$ after the second serum treatment and processed for flow cytometry by Michael Haberl (Department of Neuroimmunology, Institute for Multiple Sclerosis Research, Göttingen). FACS staining was performed for T cells (CD4 and CD8), myeloid cells (CD11b), B cells (CD45RA) and PMN (RP1).

The numbers of PMN were strongly decreased in rats treated with anti-PMN antiserum and represented $0.8 \%$ of the PMN numbers detected in normal serum treated rats. However, a reduction of immune cells $/ \mathrm{ml}$ to $5.5-8.1 \%$ of the cell numbers measured in normal serum treated rats was also observed in all leukocyte subsets investigated (Figure 13). In detail, PMN were reduced from $1.68 \pm 1.1 \times 10^{6}$ cells $/ \mathrm{ml}$ in normal serum treated animals to $0.01 \pm$ $0.01 \times 10^{6}$ cells $/ \mathrm{ml}$ in anti-PMN antiserum treated animals. Furthermore, decreased numbers of T cell were detected: CD4 positive T cells were reduced to $6.5 \%$ and CD8 positive T cells to $5.5 \%$ in anti-PMN antiserum treated animals (normal serum: $3.2 \pm 1.5 \times 10^{5} \mathrm{CD} 4+\mathrm{T}$ cells $/ \mathrm{ml}$ and $2.1 \pm 0.2 \times 10^{5} \mathrm{CD} 8+\mathrm{T}$ cells $/ \mathrm{ml}$; anti-PMN: $0.21 \times 10^{5} \pm 0.13 \times 10^{5} \mathrm{CD} 4+\mathrm{T}$ cells $/ \mathrm{ml}$ and $0.12 \times 10^{5} \pm 0.03 \times 10^{5} \mathrm{CD} 8+\mathrm{T}$ cells $\left./ \mathrm{ml}\right)$. B cell numbers were decreased from $1.10 \pm 0.55 \times$ $10^{5} \mathrm{~B}$ cells $/ \mathrm{ml}$ in normal serum treated animals to $0.09 \pm 0.08 \times 10^{5} \mathrm{~B}$ cells $/ \mathrm{ml}$ in anti-PMN antiserum treated rats, which represents a reduction to $8.1 \%$. In addition, CD11b positive myeloid cells decreased to $6.6 \%$ after anti-PMN antiserum treatment (normal serum: $7.03 \pm$ $2.99 \times 10^{4} \mathrm{CD} 11 \mathrm{~b}$ positive cells/ml; anti-PMN antiserum: $0.47 \pm 0.02 \times 10^{4} \mathrm{CD} 11 \mathrm{~b}$ positive cells/ml). 

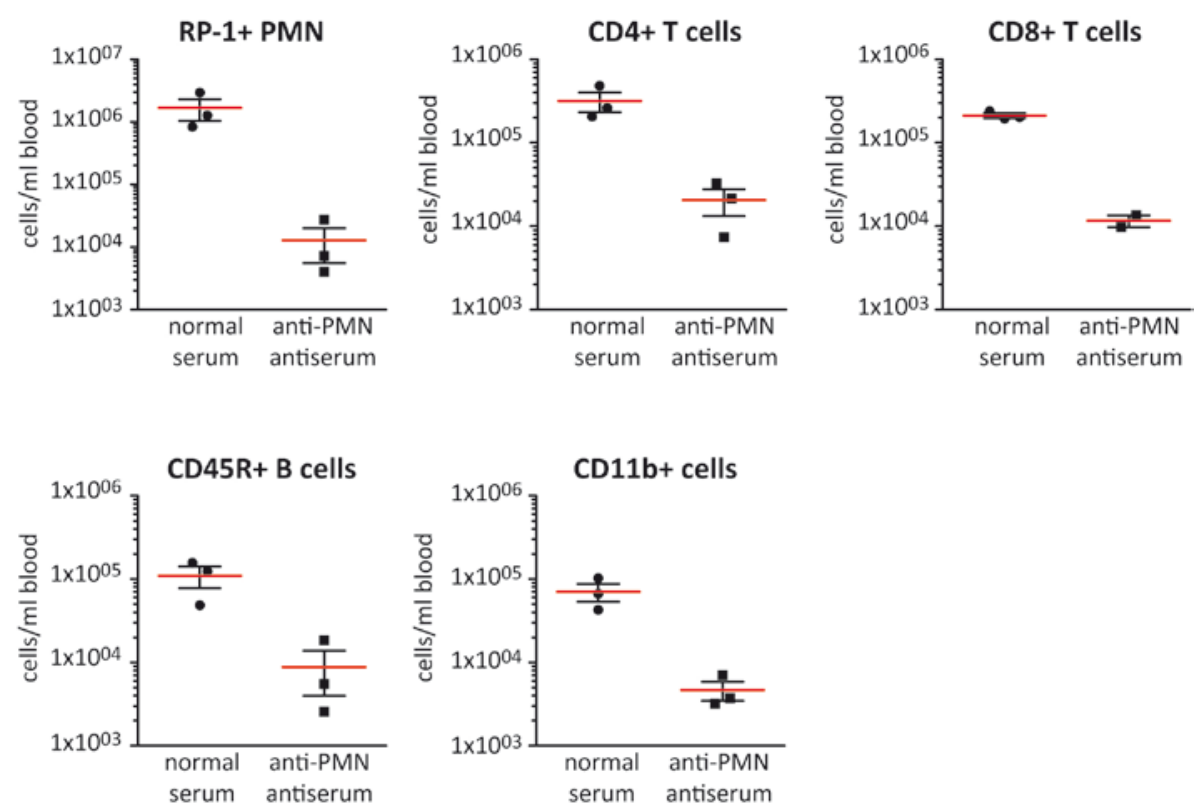

Figure 13: Treatment of animals with anti-PMN antiserum resulted in a strong decrease of PMN numbers but also a non-selective reduction of leukocytes compared to normal serum treated animals

Treatment of rats with anti-PMN antiserum strongly decreased the numbers of RP- 1 positive PMN to $0.8 \%$ in relation to the PMN number detected after treatment with normal serum. However, the depletion of PMN with anti-PMN antiserum was not selective. In addition, reduced numbers of CD4 and CD8 positive T cells, CD45R positive $B$ cells and CD11b positive myeloid cells were observed after anti-PMN antiserum treatment. $n$ in each group $=3$

\subsection{Inhibition of complement component C5a receptor (C5aR) significantly reduces astrocyte lesion size $6 \mathrm{~h}$ after lesion induction}

The development of focal NMO-like lesions is complement-dependent. After injection of $\mathrm{NMO}-\mathrm{Ab}$ and human complement, lesion development is triggered by the binding of the NMO-Ab to AQP4 on astrocytes and the subsequent activation of the complement cascade which results in the lysis of astrocytes (Wrzos et al., 2014). During the activation of the complement cascade, C5 convertase becomes activated and cleaves the inactive C5 into the active component $\mathrm{C} 5 \mathrm{~b}$ and the anaphylatoxin $\mathrm{C} 5 \mathrm{a}$. Its receptor $\mathrm{C} 5 \mathrm{aR}$ is highly expressed on PMN. C5a acts as a potent PMN chemoattractant, but is also important for PMN activation. In order to inhibit C5aR mediated signaling the small molecule C5aR antagonist PMX-53 was used. Rats were focally injected with NMO-Ab together with human complement and were i.v. injected with $1 \mathrm{mg} / \mathrm{kg}$ body weight PMX-53 or vehicle $3 \mathrm{~h}$ later. Animals were sacrificed $6 \mathrm{~h}$ after lesion induction (Figure $14 \mathrm{~A}$ ). For assessment of BBB integrity FITC-albumin was i.v. injected $40 \mathrm{~min}$ before perfusion. Immunohistochemistry was used to investigate astrocyte lesion size (GFAP and AQP4) and BBB permeability (FITC-albumin and fibrinogen). Additionally, CAE enzyme histochemistry was performed to visualize PMN. 
Focal injection of NMO-Ab and human complement results in the formation of astrocyte depleted lesions with sizes of $0.4 \pm 0.2 \mathrm{~mm}^{2}$ (AQP4) and $0.4 \pm 0.1 \mathrm{~mm}^{2}$ (GFAP) in vehicle treated animals. Systemic treatment with the C5aR antagonist PMX-53 significantly reduced the size of astrocyte depleted lesions (AQP4: $0.2 \pm 0.2 \mathrm{~mm}^{2},{ }^{*} p=0.0291$ and GFAP: $0.2 \pm 0.2$ $\mathrm{mm}^{2},{ }^{* *} \mathrm{p}=0.0039$, Figure $\left.14 \mathrm{~B}\right)$. The infiltration of PMN into the brain parenchyma $6 \mathrm{~h}$ after lesion induction, however, was not significantly altered between vehicle and PMX-53 treated animals (vehicle: $538 \pm 262 \mathrm{PMN} / \mathrm{mm}^{2}, \mathrm{PMX}-53: 329 \pm 231 \mathrm{PMN} / \mathrm{mm}^{2}$, Figure $14 \mathrm{C}$ ). Furthermore, no difference in BBB permeability was observed. The area of FITC-albumin extravasation was $1.1 \pm 0.6 \mathrm{~mm}^{2}$ in vehicle and $1.0 \pm 0.6$ in PMX-53 treated animals (Figure 14 D). Similar values were obtained for the area of fibrinogen extravasation (vehicle: $1.3 \pm$ $0.8 \mathrm{~mm}^{2}, \mathrm{PMX}-53: 1.1 \pm 0.6 \mathrm{~mm}^{2}$, Figure $\left.14 \mathrm{E}\right)$.

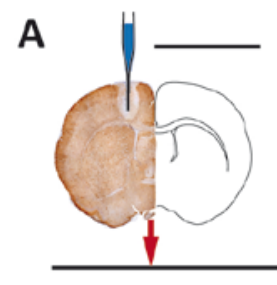

B
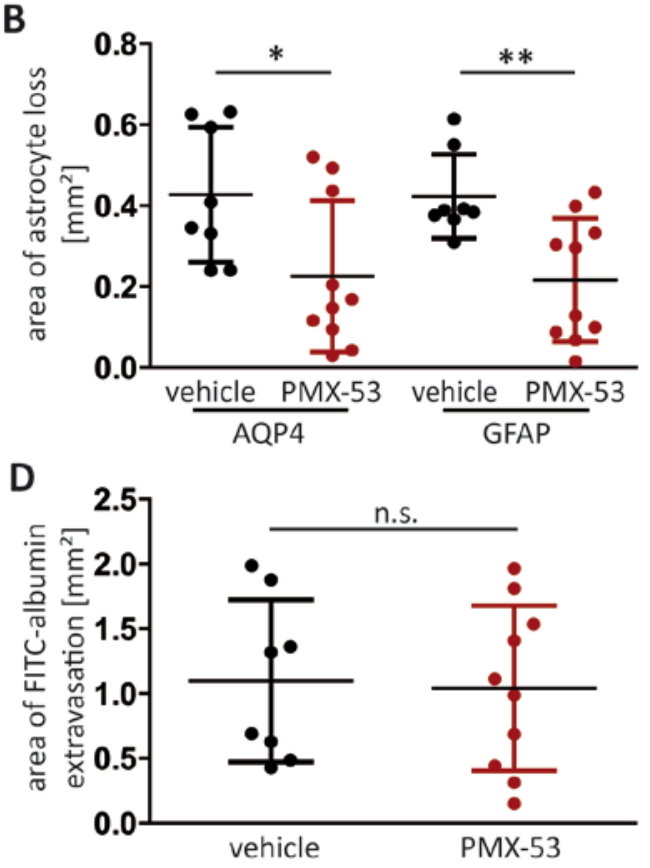

NMO-Ab

together with

human complement
$1 \mathrm{mg} / \mathrm{kg}$ PMX-53 or vehicle i.v.

$3 h$

C
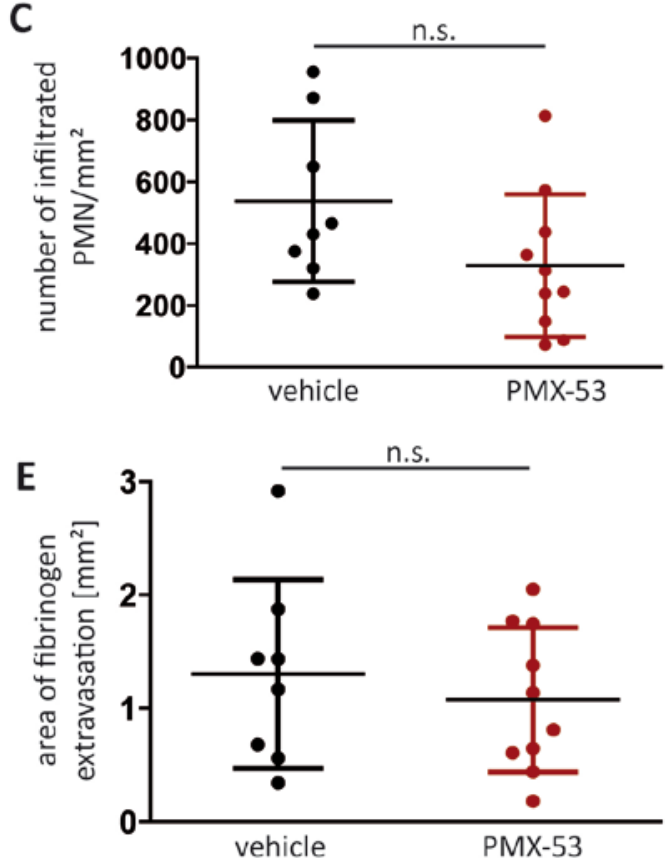

Figure 14: Decreased astrocyte loss after systemic treatment with the C5aR antagonist PMX-53 $6 \mathrm{~h}$ after lesion induction

Schematic illustration of the experimental setup to investigate the influence of the C5aR antagonist PMX-53 on the development of NMO-like lesions is depicted in (A). Treatment of rats with the C5aR antagonist PMX-53 significantly decreased the areas with loss of GFAP and AQP4 immunoreactivity compared to vehicle treated animals (B). However, infiltration of PMN was not altered in PMX-53 compared to vehicle treated animals (C). Additionally, no effect of PMX-53 treatment on the preservation of BBB integrity was observed, which was assessed by the measurement of FITC-albumin (D) and fibrinogen extravasation (E). Vehicle treated animals $n=8$, PMX-53 treated animals $n=10$, pooled data of 2 independent experiments. Unpaired t-test with Welch's correction, $* p<0.05, * * p<0.01$, n.s. $=$ not significant. Data are shown as mean \pm SD. 


\subsection{Investigation of PMN granule proteases in the regulation of BBB permeability}

PMN interactions with the endothelium under inflammatory conditions can result in the dysfunction of the BBB and an increased permeability. Several secreted PMN products have been implied to increase the endothelial permeability, like the proteases cathepsin $G$, neutrophil elastase (NE) and matrix metalloproteinase 9 (MMP9) (Chen et al., 2009; Hermant et al., 2003; Suzuki et al., 1994). In order to assess the direct effect of these proteases on brain endothelial cells, in vitro experiments were performed. Furthermore, the localization of MMP9 in infiltrating PMN and the effect of NE inhibition were investigated in vivo.

\subsubsection{Treatment of RBEC with MMP9 decreases electrical resistance in vitro}

PMN granules contain proteases like cathepsin G, NE and MMP9 which are released upon PMN activation. In order to investigate a direct effect of these proteases on the TEER values of confluent RBEC monolayers, cathepsin G $(0.06 \mathrm{U} / \mathrm{ml})$, NE $(6 \mu \mathrm{g} / \mathrm{ml})$ or MMP9 $(0.5 \mu \mathrm{g} / \mathrm{ml})$ were added to the apical compartment of a transwell system containing RBEC (Figure $15 \mathrm{~A}$ ). TEER was measured before treatment and after $60 \mathrm{~min}$ and $120 \mathrm{~min}$. Untreated RBEC monolayers were used as controls. To exclude a direct cytotoxic effect of the proteases on RBEC, the lactate dehydrogenase (LDH) based CytoTox-ONE ${ }^{\mathrm{TM}}$ Homogeneous Membrane Integrity Assay was applied. To assess the localization of occludin and claudin-5 in RBEC after treatment with MMP9 immunocytochemistry was performed.

Before treatment TEER values of $41 \pm 2 \Omega * \mathrm{~cm}^{2}$ across RBEC monolayers were measured. Treatment of RBEC with neither cathepsin G nor NE decreased TEER. In detail, in cathepsin G treated cells TEER values of $37 \Omega^{*} \mathrm{~cm}^{2}(60 \mathrm{~min})$ and $36 \Omega^{*} \mathrm{~cm}^{2}(120 \mathrm{~min})$ were determined. Treatment with NE resulted in an electrical resistance of $39 \Omega^{*} \mathrm{~cm}^{2}$ (60 min) and $35 \Omega * \mathrm{~cm}^{2}$ (120 $\mathrm{min}$ ). Both did not differ to untreated controls $\left(60 \mathrm{~min}: 38 \Omega^{*} \mathrm{~cm}^{2}\right.$ and $120 \mathrm{~min}: 38$ $\left.\Omega^{*} \mathrm{~cm}^{2}\right)$. Compared to untreated controls, however, MMP9 treatment of RBEC strongly decreased TEER values at both time points $\left(23.4 \Omega^{*} \mathrm{~cm}^{2}(60 \mathrm{~min})\right.$ and $19.5 \Omega^{*} \mathrm{~cm}^{2}(120 \mathrm{~min})$, Figure 15 B). To assess a possible cytotoxic effect of cathepsin G, NE or MMP9 treatment on RBEC, the release of LDH by damaged cells as a biomarker for cellular cytotoxicity and cytolysis was measured after $120 \mathrm{~min}$ of treatment. Compared to untreated controls no increase in LDH levels/cytotoxicity was observed after treatment with cathepsin G, NE or MMP9, confirming that a non-cytotoxic protease concentration was used for the transwell experiments (Figure $15 \mathrm{C}$ ). To investigate if treatment of RBEC with MMP9 results in loss of 
occludin or claudin-5 from the TJ at the endothelial cell contacts, immunocytochemistry was performed. In controls, occludin and claudin-5 immunocytochemistry was localized at the TJ between adjacent RBEC forming defined strands. No difference in staining pattern was observed after 120 min of incubation with MMP9 (Figure 15 D).
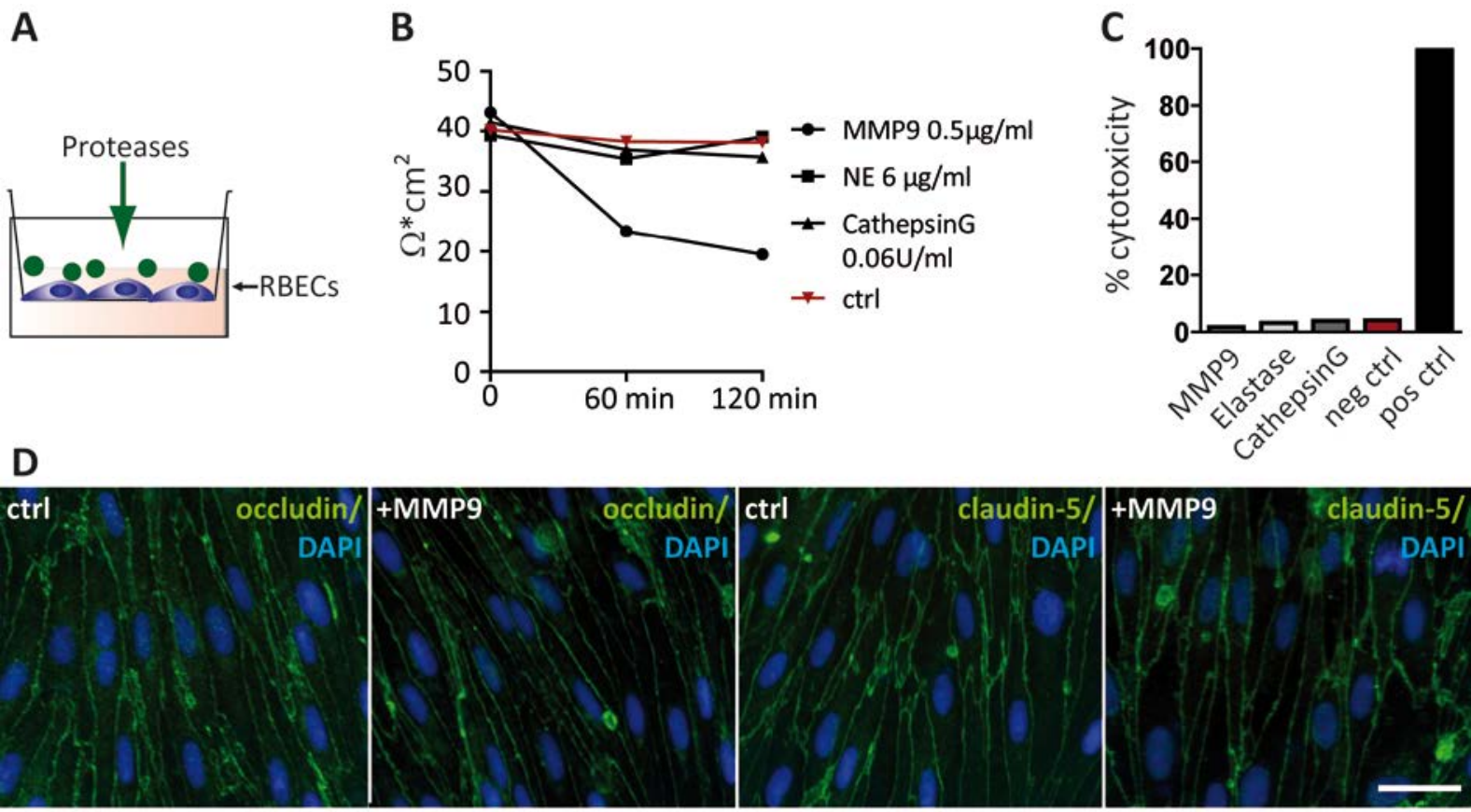

Figure 15: Treatment of RBEC with MMP9, but not NE or cathepsin G, reduced TEER in vitro

To investigate the effect of neutrophil proteases on BBB permeability, RBEC were incubated with cathepsin G, NE or MMP9 in vitro using a transwell system (A). Treatment of RBEC with MMP9 for 60 min and 120 min decreased TEER values while treatment with NE or cathepsin G did not alter TEER compared to untreated controls (B). To exclude a direct cytotoxic effect of MMP9, cathepsin G and NE on the RBEC, a LDH based assay for cytotoxicity was performed and the release of LDH by damaged cells as a biomarker for cellular cytotoxicity was measured. LDH release of the positive (pos) ctrl (RBEC treated with lysis buffer) was set to $100 \%$ cytotoxicity. Compared to untreated (neg) ctrl no increase in LDH levels/cytotoxicity was detected in RBEC treated with MMP9 $(0.5 \mu \mathrm{g} / \mathrm{ml})$, cathepsin $\mathrm{G}(0.06 \mathrm{U} / \mathrm{ml})$ or NE $(6 \mu \mathrm{g} / \mathrm{ml})$. (C). Immunocytochemistry for occludin and claudin-5 demonstrated localization of both TJ proteins between adjacent cells in untreated controls. No difference of occludin and claudin-5 immunoreactivity was observed after treatment with MMP9 (D). Scale bar $50 \mu \mathrm{m}$

\subsubsection{MMP9 positive PMN are abundant in NMO-like lesions $6 \mathrm{~h}$ after lesion induction}

In order to investigate MMP9 expression in infiltrating PMN after lesion induction, a time course study was performed. Animals were focally injected with NMO-Ab and human complement and subsequently sacrificed $6 \mathrm{~h}, 12 \mathrm{~h}, 24 \mathrm{~h}$ and $3 \mathrm{~d}$ after lesion induction. Immunohistochemistry for MMP9 was performed. PMN were identified based on their multi-lobed nuclear morphology in Mayer's hemalum counterstain. The total number of PMN was determined as well as the number of PMN with cellular MMP9 immunoreactivity to calculate the percentage of MMP9 positive PMN. 
$6 \mathrm{~h}$ after lesion induction, in $19.3 \pm 10.9 \%$ of PMN MMP9 immunoreactivity was observed which was localized within the cells in a granular pattern (Figure $16 \mathrm{~A}$, arrowheads; C). Subsequently, a reduction of PMN with intracellular MMP9 immunoreactivity was detected. $12 \mathrm{~h}$ after lesion induction $10.4 \pm 8.7 \%$ of PMN were MMP9 positive which was reduced further to $1.7 \pm 1.4 \% 24 \mathrm{~h}$ after lesion induction (Figure $16 \mathrm{~B}, \mathrm{C}$ ). No MMP9 positive cells were observed $3 \mathrm{~d}$ after lesion induction (Figure $16 \mathrm{C}$ ).
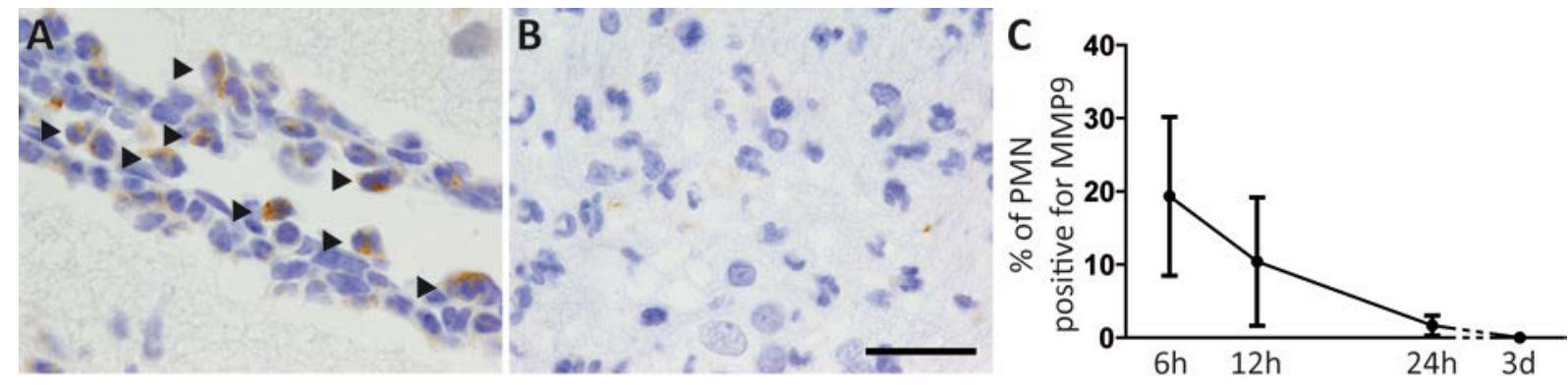

Figure 16: Intracellular MMP9 expression was detected in infiltrating PMN 6 h and 12 h after lesion induction

Numerous vascular and extravasating PMN displayed granular intracellular staining for MMP9 $6 \mathrm{~h}$ after lesion induction (arrowheads, A). Rare MMP9 positive PMN were detected $24 \mathrm{~h}$ after lesion induction (B). Quantification of MMP9 positive PMN in the lesions displayed a continuous decrease of MMP9 immunoreactivity within PMN. No MMP9 positive cells were observed $3 \mathrm{~d}$ after lesion induction $6 \mathrm{~h} n=4,12 \mathrm{~h}$ $n=2,24 \mathrm{~h} n=4,3 \mathrm{~d} n=3$, C). Data are shown as mean \pm SD. Scale bar $20 \mu \mathrm{m}$

\subsubsection{Inhibition of NE reduces astrocyte lesion size and PMN infiltration $6 \mathrm{~h}$ after lesion induction}

NE did not show a direct effect on TEER values of RBEC in vitro (Figure 15). However, in vitro and in vivo data indicate that NE cleaves the intercellular adhesion molecule 1 (ICAM-1) and thereby may play a role in PMN extravasation in vivo (Champagne et al., 1998; Kaynar et al., 2008). To investigate the role of NE in the development of focal NMO-like lesions, the competitive NE inhibitor Sivelestat was used. Rats were intraperitoneal (i.p.) injected with $50 \mathrm{mg} / \mathrm{kg}$ body weight Sivelestat or vehicle $3 \mathrm{~h}$ after focal injection of NMO-Ab and human complement. Subsequently, animals were sacrificed $6 \mathrm{~h}$ after lesion induction (Figure $17 \mathrm{~A}$ ). For evaluation of the BBB integrity FITC-albumin was injected i.v. 40 min before perfusion. Immunohistochemistry was used to investigate astrocyte loss (GFAP and AQP4) and FITCalbumin extravasation. Furthermore, CAE enzyme histochemistry was performed to visualize PMN.

In vehicle treated animals astrocyte depleted lesions developed $6 \mathrm{~h}$ after lesion induction (AQP4: $0.3 \pm 0.2 \mathrm{~mm}^{2}$, GFAP: $0.3 \pm 0.2 \mathrm{~mm}^{2}$ ). Treatment with the NE inhibitor Sivelestat significantly reduced the area of astrocyte loss (AQP4: $0.1 \pm 0.1 \mathrm{~mm}^{2},{ }^{*} \mathrm{p}=0.0184$ and GFAP: 
$0.1 \pm 0.1 \mathrm{~mm}^{2}, * \mathrm{p}=0.0415$, Figure $17 \mathrm{~B}$ ). Furthermore, the density of infiltrated PMN in the brain parenchyma was significantly decreased in Sivelestat treated animals compared to vehicle treated controls (vehicle: $603 \pm 376 \mathrm{PMN} / \mathrm{mm}^{2}$, Sivelestat: $256 \pm 154 \mathrm{PMN} / \mathrm{mm}^{2}$, $*_{p}=0.0387$, Figure $\left.17 \mathrm{C}\right)$. However, no difference in FITC-albumin extravasation was observed between vehicle and Sivelestat treated animals (vehicle: $1.0 \pm 0.6 \mathrm{~mm}^{2}$, Sivelestat: $0.9 \pm 0.6$ $\mathrm{mm}^{2}$, Figure $\left.17 \mathrm{D}\right)$.

A

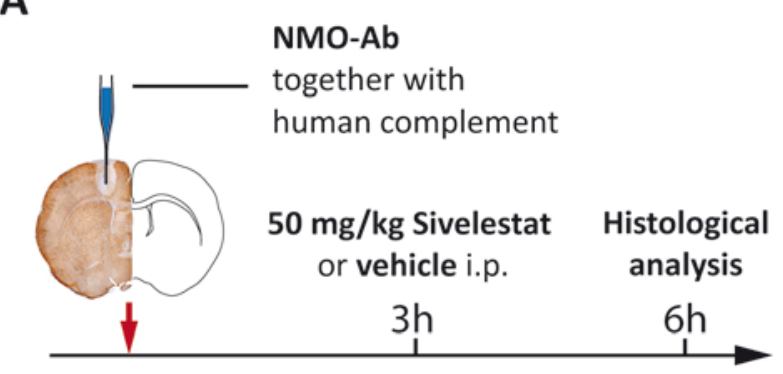

C

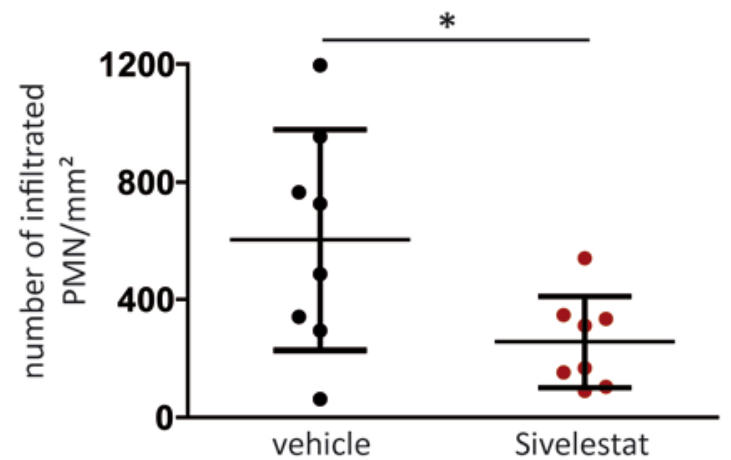

B

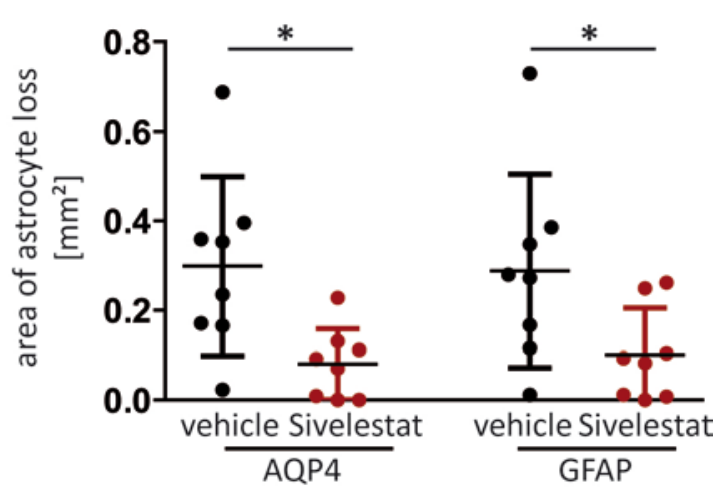

D

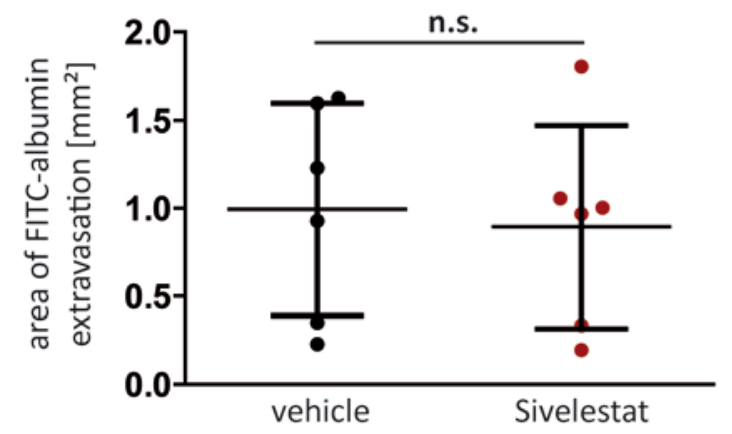

Figure 17: Inhibition of NE using Sivelestat resulted in a significant reduction of astrocyte lesion size and PMN infiltration $6 \mathrm{~h}$ after lesion induction

Illustration of the experimental setup to assess the influence of NE inhibition using Sivelestat in vivo (A). Treatment of animals with $50 \mathrm{mg} / \mathrm{kg}$ Sivelestat resulted in a significant reduction in the size of astrocyte depleted lesion compared to vehicle treated animals measured in AQP4 and GFAP immunohistochemistry (B). In addition, treatment with Sivelestat significantly decreased infiltration of PMN (C). Compared to vehicle treated animals no alterations in BBB permeability were detected after treatment with Sivelestat (D). Unpaired t-test with Welch's correction, ${ }^{*} p<0.05$, n.s. $=$ not significant. B, C: $n=8 ;$ D: $n=6$, pooled data of two independent experiments. Data are shown as mean $\pm \mathrm{SD}$.

\subsection{PMNs are a component of the inflammatory infiltrate in early human NMO lesions}

In focal NMO-like lesions PMN play a crucial role in the regulation of BBB permeability and lesion development. Therefore, the immune cell infiltrate of early human NMO lesions was investigated. Early active human NMO lesions were characterized by focal loss of AQP4 and GFAP immunoreactivity, focal loss of Luxol fast blue (LFB) positive myelin sheets and relative axonal preservation (as depicted in Figure 1). Five biopsies and two autopsies from anti- 
AQP4 seropositive patients with confirmed NMO or NMO spectrum disorder were assessed. To visualize immune cell subsets immunohistochemistry against MRP14 (recently invaded monocytes/macrophages and PMN), KIM1P (macrophages/activated microglia) and CD3 (T cells) was performed. Infiltrating PMN numbers were evaluated in hematoxylin and eosin (HE) staining based on their multi-lobed nuclear morphology.

A density of $132 \pm 89 \mathrm{PMN} / \mathrm{mm}^{2}$ (Figure $18 \mathrm{~A}, \mathrm{E}, \mathrm{F}$ (red dots)) and $465 \pm 291 \mathrm{MRP} 14$ positive cells $/ \mathrm{mm}^{2}$ (Figure $18 \mathrm{~B}, \mathrm{~F}$ (red dots)) was detected in biopsy tissue. These numbers were lower in autopsy tissue with a density of $2 \pm 2 \mathrm{PMN} / \mathrm{mm}^{2}$ (Figure $18 \mathrm{~F}$ (black dots)) and $45 \pm$ 63 MRP14 positive cells $/ \mathrm{mm}^{2}$ (Figure $18 \mathrm{~F}$ (black dots)). This difference might be due to the disease phase the tissue was collected. Although we investigated autopsy material with active, inflammatory NMO lesions, it is likely that these lesions reflect a more advanced (subacute) stage of NMO-lesion development, also considering the fact that most diagnostic biopsies are taken during acute disease phases. Furthermore, CD3 positive infiltrated T cells (95 \pm 97 cells $/ \mathrm{mm}^{2}$, Figure $18 \mathrm{D}, \mathrm{F}$ ) and abundant numbers of KiM1P positive macrophages/activated microglia (1195 \pm 241 cells $/ \mathrm{mm}^{2}$, Figure $18 \mathrm{C}, \mathrm{F}$ ) were observed in both autopsies and biopsies. 


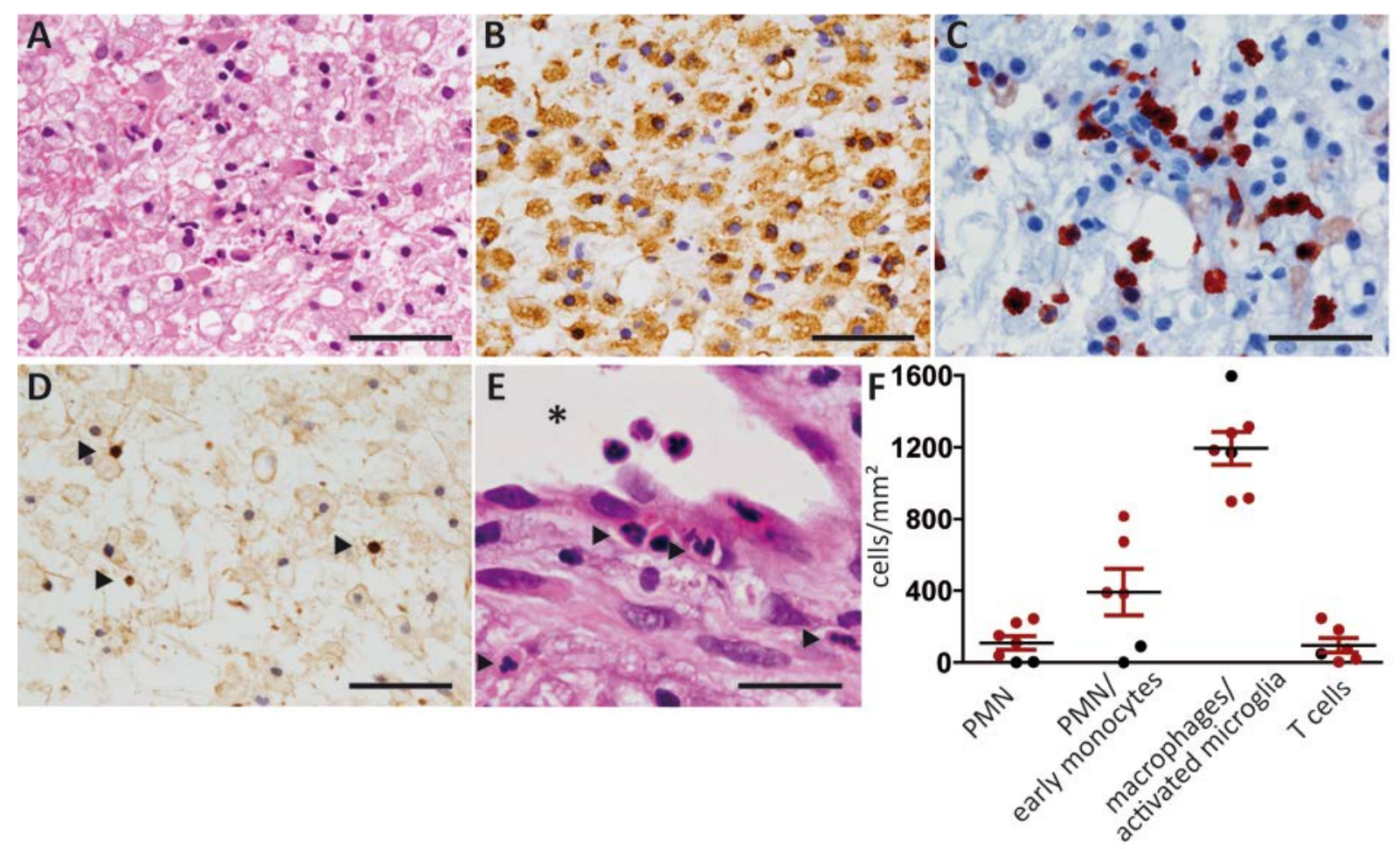

Figure 18: In the inflammatory infiltrate of early human NMO lesions PMN, monocytes, macrophages/activated microglia and T cells were observed

Inflammatory infiltrates were observed in early active NMO lesions. These infiltrates were characterized by PMN (HE staining, A and higher magnification: E. Infiltrated PMN are indicated by arrows. The asterisk marks the lumen of a blood vessel from which PMN extravasate). In addition, PMN and early infiltrating monocytes were visualized by MRP14 immunohistochemistry (B). Furthermore, early human NMO lesions were characterized by abundant KiM1P positive macrophages/activated microglia (C) and CD3 positive T cells (arrows, D). Quantification of immune cells is depicted in (F). F: red: biopsies $(n=5)$; black: autopsies $(n=2)$. Cell numbers were evaluated using the following staining methods: PMN: HE; early monocytes/PMN: MRP14;

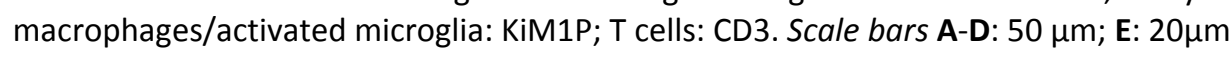


Neuromyelitis optica (NMO) is a demyelinating disease in which astrocytes are the major target. Indeed, about 70\% of NMO patients were found to be seropositive for antibodies (Ab) against aquaporin4 (AQP4) (Jarius et al., 2012b; Lennon et al., 2005; Lennon et al., 2004; Mealy et al., 2012; Waters et al., 2012) a water channel which is highly expressed on astrocytic endfeet in the central nervous system. Binding of this antibody to AQP4 on the astrocyte surface results in extensive complement and cell mediated astrocyte depletion during the acute phase of NMO lesion development. Astrocytes form, together with endothelial cells, pericytes and the basal lamina, the blood-brain barrier (BBB). Indeed, NMO patients manifest with a severe BBB disruption, as evidenced by multiple gadolinium enhanced lesions on magnetic resonance imaging (MRI) (de Seze et al., 2002; Ito et al., 2009; Kim et al., 2015; Wingerchuk et al., 1999). Furthermore, it was demonstrated that the extent of BBB permeability (measured by cerebrospinal fluid (CSF)/serum albumin ratios) correlated with the clinical disability (Tomizawa et al., 2012). Since the close interaction between different components of the BBB is necessary for maintaining its integrity, we hypothesized that the loss of astrocytes from the BBB in NMO lesions contributes to the severe BBB dysregulation that is observed in NMO patients. Therefore, the aims of this project were to characterize the breakdown of the $\mathrm{BBB}$ in an animal model of $\mathrm{NMO}$ in detail and to investigate its underlying mechanisms. In particular we were interested to examine whether there is (i) a correlation between BBB breakdown and astrocyte loss, (ii) a correlation between BBB breakdown and the disruption of tight junctions (TJ) at the BBB and (iii) a possible function of immune cells in the induction of BBB permeability.

In the present study we showed, that (i) onset of the development of astrocyte depleted lesions correlated with the time point of BBB breakdown in vivo. However, the BBB integrity was restored before astrocytes repopulated the lesion as observed using vascular tracers. Additionally co-culture of rat brain endothelial cells (RBEC) with astrocytes did not increase transendothelial electrical resistance (TEER) in vitro. No loss of pericytes from astrocyte depleted lesions was observed.

Furthermore, this work demonstrated that (ii) the TJ molecule occludin is selectively lost from the TJ in astrocyte depleted lesions but also reappears before astrocytes repopulate. Surprisingly, the BBB integrity to vascular tracer molecules was re-established before occludin immunoreactivity was again localized at the TJ. However, claudin-3 and -5 localization at the TJ was not affected at any time point investigated. Moreover, no 
morphological alterations of the TJ at endothelial contacts were detected on ultrastructural level using electron microscopy (EM). An increased mRNA expression of the TJ molecules occludin and claudin-5 was measured $10 \mathrm{~h}$ and $24 \mathrm{~h}$ after lesion induction.

Additionally, the present work aimed to investigate the role of immune cells in the development of focal NMO-like lesions (iii). $6 \mathrm{~h}$ after lesion induction polymorphonuclear cells (PMN) were the most abundant infiltrating immune cell subset and the number of PMN in the lesions correlated with the area of fluorescein isothiocyanate (FITC)-albumin extravasation. Depletion of PMN abrogated lesion development and prevented breakdown of the BBB. Furthermore, inhibition of the complement component $\mathrm{C} 5 \mathrm{a}$ receptor ( $\mathrm{C} 5 \mathrm{aR})$, which is important for PMN attraction and activation, reduced the area of astrocyte loss. Finally, it was demonstrated that proteases released from PMN granules upon activation were involved in the regulation of BBB permeability and astrocyte lesion formation. Matrix metalloproteinase 9 (MMP9) was shown to decrease TEER values of RBEC in vitro and was detected in infiltrating PMN $6 \mathrm{~h}$ and $12 \mathrm{~h}$ after lesion induction in vivo. Furthermore, inhibition of neutrophil elastase (NE) reduced astrocyte lesion size and PMN infiltration in vivo.

In summary we could show that PMN play a crucial role in the development of NMO-like lesions and mediate the breakdown of the BBB. The disruption of the BBB allows complement components to gain access to the brain parenchyma and results in the recruitment of additional immune cells. Both factors contribute to astrocyte loss in NMO-like lesions.

\subsection{Rapid restoration of the BBB in the focal NMO model, independent of loss of astrocytes and occludin}

To investigate the BBB in NMO, we utilized a focal NMO model in Lewis rats which is based on injection of a recombinant human NMO-Ab, directed against AQP4, together with human complement into the motorcortex. Intracerebral injection results in NMO-like lesions which are characterized by complement mediated astrocyte lysis followed by a rapid loss of oligodendrocytes and a delayed demyelination (Wrzos et al., 2014). Additionally, immune cell infiltration was observed, consisting mainly of PMN and macrophages. Astrocyte, oligodendrocyte and myelin loss, as well as PMN infiltration and abundant numbers of macrophages in the lesions are characteristics of early human NMO lesions (Cloys and Netsky, 1970; Lucchinetti et al., 2002; Misu et al., 2007; Roemer et al., 2007), see also Figure 
1 and Figure 18. Therefore, focal NMO-like lesions resemble early human NMO lesions in many aspects, especially since the NMO-Ab utilized is derived from a plasma cell of an NMO patient (Bennett et al., 2009). Furthermore, the usage of a recombinant human NMO-Ab for lesion induction - in contrast to patient sera - ensures the induction of reproducible NMOlike lesions which present a valuable tool to study astrocyte lesion development, also with regard to the regulation of the BBB permeability in the absence of astrocytes.

The $\mathrm{BBB}$ restricts the movements of serum molecules from the blood into the brain and thereby maintains the unique central nervous system (CNS) environment which enables neuronal function. It is well established that "typical" BBB properties of endothelial cells (EC), such as the development of tight interendothelial junctions or the polarized expression of transporter systems, depend on cellular and structural components of the CNS. This includes astrocytes, pericytes and the basal lamina, and in the broader term of the neurovascular unit (NVU) additionally microglia and neurons (Abbott et al., 2006). The restriction of the transendothelial flux of sodium ions across the endothelium is a feature of the BBB that is mainly dependent of the development of tight interendothelial junctions, and can be determined by the measurement of the electrical resistance. TEER values of about $1800 \Omega * \mathrm{~cm}^{2}$ were measured in situ in the rat CNS, representing a tight barrier (Butt et al., 1990). However, once isolated, the TEER values decrease to $50-150 \Omega * \mathrm{~cm}^{2}$ in monolayers of rat brain EC (e.g. de Vries et al., 1996; Demeuse et al., 2002; Perriere et al., 2007). Co-culture of brain endothelial monolayers with astrocytes and/or pericytes was shown to increase the TEER values, to reduce permeability and to result in an enhanced expression of TJ proteins (Demeuse et al., 2002; Nakagawa et al., 2009; Nakagawa et al., 2007; Perriere et al., 2007).

In order to identify a possible temporal relationship between astrocyte loss and the disruption of the BBB in focal NMO-like lesions a detailed time course study was performed. We detected dying glial fibrillary acidic protein (GFAP) positive astrocytes $3 \mathrm{~h}$ after focal injection of NMO-Ab and human complement. $6 \mathrm{~h}$ after lesion induction well-demarcated areas of GFAP loss were observed (Figure 3). Subsequently, loss of GFAP immunoreactivity expanded until lesions developed the maximal extent between $24 \mathrm{~h}$ and $3 \mathrm{~d}$. Loss of astrocytes was also confirmed by immunohistochemistry against the astrocytic markers AQP4 (Figure 3), S100 calcium-binding protein $ß$ (S100ß) and excitatory amino-acid transporter 2 (EAAT2) (Wrzos et al., 2014). One week after lesion induction $91.1 \%$ of the formerly astrocytes depleted area was repopulated by GFAP positive astrocytes (Figure 3). No loss of GFAP positive astrocytes was observed in animals with focal injection of a CNS 
unspecific control (ctrl)-Ab and human complement (Figure 3). Additionally, previous work of our group shows that intracerebral injection of NMO-Ab together with heat-inactivated human serum does not induce astrocyte loss (Wrzos et al., 2014). Concurrent with the development of well-demarcated astrocyte depleted lesions, a transient disruption of the BBB to the endogenous serum proteins immunoglobulin $G(\operatorname{lgG})(150 \mathrm{kDa})$ and fibrinogen (340 kDa, Figure 4) and the vascular tracer molecules (FITC)-albumin (70 kDa) and Texas $\operatorname{Red}^{\circledR}$ cadaverine $(0.69 \mathrm{kDa}$, Figure 5$)$ was detected $6 \mathrm{~h}$ after lesion induction. Surprisingly, $24 \mathrm{~h}$ after lesion induction the BBB integrity was restored as observed for the vascular tracers FITC-albumin and Texas $\operatorname{Red}^{\circledR}$ cadaverine although the highest extent of astrocyte loss was measured at that time point (Figure 1 and Figure 5).

Although an improvement of the BBB phenotype of isolated brain EC has been described when co-cultured with astrocytes, only few studies are published that directly investigate the loss of astrocytes and its effect on the BBB integrity in the adult brain in vivo. Early transplantation studies suggest that astrocyte presence alone does not induce a BBB phenotype in vessels. When fetal CNS tissue was transplanted into cerebral ventricles and subsequently was penetrated by choroidal vessels, these vessels were permeable for tracer molecules although ensheated by astrocytes (Krum and Rosenstein, 1989; Rosenstein, 1987). To the contrary, it was shown that transplantation of cultured astrocytes but not fibroblasts into the anterior eye chamber of rats resulted in protein impermeable vessels which vascularized the graft originating from the iris (Janzer and Raff, 1987). However, the iris microvasculature is characterized by an ocular-brain barrier and subsequent studies showed the astrocyte graft to be poorly vascularized in contrast to the fibroblast graft in which additionally a significant inflammatory response was observed (Holash et al., 1993). Later, using 3-chloropropanediol in rats to selectively deplete astrocytes in a toxic model, it was demonstrated that astrocyte loss precedes the breakdown of the BBB to FITC-labeled dextran. However, similar to the results obtained in our experiments, the integrity of the BBB to tracer molecules > $10 \mathrm{kDa}$ was restored in the absence of astrocytes (Willis et al., 2004). This restoration of the BBB integrity was later attributed to the continued expression of adherens junction (AJ) molecules, a remodeling of the extracellular matrix and an infiltration of macrophages (Willis et al., 2013). In another toxic model using the glial toxin $\mathrm{N}$-aminonicotinamine, Krum and colleague investigated the effect of astrocyte depletion on the endothelial BBB phenotype in a series of studies. They demonstrated that depletion of astrocytes in the brain did not result in a breakdown of the BBB to horseradish peroxidase or serum albumin in neonatal rats (Krum and Rosenstein, 1993). In adult animals, injection of 
$\mathrm{N}$-aminonicotinamine did not result in astrocyte death in the brain but in the spinal cord. Here, extravasation of horseradish peroxidase was observed from some, but not all astrocyte depleted vessels (Krum, 1994). In addition the expression of some microvascular markers (e.g. the glucose transporter 1 (Glut1)) stayed intact in the absence of astrocytes (Krum, 1994, 1996). In a genetic approach using mice expressing the herpes simplex virus thymidine kinase (HSV-TK) under the GFAP promoter, it was shown that ganciclovir mediated depletion of reactive, GFAP expressing astrocytes after a forebrain stab injury resulted in a prolonged opening of the BBB. In addition an increase and prolonged duration of leukocyte infiltration (including PMN, macrophages, monocytes and lymphocytes) was observed. However, it was not investigated whether the depletion of astrocytes or the increase in inflammatory cell infiltration were causing the prolonged disruption of BBB integrity (Bush et al., 1999).

Therefore, the direct effect of the loss of astrocytes on the maintenance of the BBB in the adult brain is not yet completely understood. In addition it was shown that astrocytic activation, e.g. during neuroinflammation, can lead to the release of signaling molecules by astrocytes which modulate BBB permeability and the expression of cell adhesion molecules on the endothelial surface. This was demonstrated e.g. for the release of sonic hedgehog (Shh) (Alvarez et al., 2011), vascular endothelial growth factor A (VEGF-A) (Argaw et al., 2009) and retinoic acid (Mizee et al., 2014). Our observation that astrocyte loss outlasts the loss of BBB integrity might be partially explained by the fact that in focal NMO-like lesions only a transient loss of astrocytes in a relatively small (about $1 \mathrm{~mm}^{2}$ in diameter) and restricted area of the CNS is observed. To further investigate the influence of factors released by primary astrocytes on TEER values of RBEC, we established an in vitro model of the BBB in which both cell types were co-cultured in separate compartments of a transwell system. However, co-culture of RBEC with astrocytes did not result in an increase of electrical resistance compared to RBEC cultured alone (Figure $6 \mathrm{C}$ ). Further experiments are planned in which the effect of astrocytes on the permeability of RBEC monolayers will be investigated using soluble tracer molecules in this experimental setup. In addition, to further dissect the effect of astrocyte loss on the integrity of the BBB additional studies are planned using conditional transgenic mouse models where focal loss of astrocytes can be induced e.g. by using GFAP Cre-inducible diphtheria toxin receptor (iDTR) mice.

Beside astrocytes, pericytes are important for the maintenance of endothelial BBB characteristics. As mentioned above, co-culture with pericytes enhances BBB properties of 
brain EC in vitro. Recent in vivo experiments underline the importance of pericytes in the establishment and maintenance of the BBB (Armulik et al., 2010; Bell et al., 2010; Daneman et al., 2010b). Using transgenic mice with significantly decreased vessel coverage by pericytes, an increase in BBB permeability (Armulik et al., 2010; Bell et al., 2010) and age dependent reduction of TJ protein expression (Bell et al., 2010) were observed in adult animals. Using the pericyte marker platelet-derived growth factor receptor $§$ (PDGFRß) we demonstrated that PDGFRß immunopositive cells line brain vessels in perilesional brain tissue (Figure $7 \mathrm{~A}$ ). This localization was not altered within astrocyte depleted areas $6 \mathrm{~h}$ and $24 \mathrm{~h}$ after lesion induction (Figure $7 \mathrm{~B}, \mathrm{C}$ ). This indicates that pericytes are not lost after lesion induction and therefore pericyte - EC crosstalk might contribute to the rapid restoration of the BBB integrity to soluble molecules after the PMN mediated BBB disruption in the absence of astrocytes.

An important structure which regulates the paracellular diffusion of soluble molecules into the brain parenchyma are TJ strands which are located between adjacent brain EC and seal the paracellular cleft. Although the TJ structure itself was already described in 1967 using EM (Reese and Karnovsky, 1967), the first TJ transmembrane linker protein occludin was only discovered in 1993 (Furuse et al., 1993). Besides occludin, another family of transmembrane linker proteins has been described: the claudins which are the main structural TJ components and consist to date of 27 members (Mineta et al., 2011). Claudin-1, $-3,-5$ and 12 have been described to be located at the TJ within the brain (Liebner et al., 2000b; Nitta, 2003; Wolburg et al., 2003). A disruption of the TJ morphology or loss of TJ proteins from the vasculature has been implicated in the breakdown of the BBB, e.g. in MS or glioblastoma (Kirk et al., 2003; Liebner et al., 2000a; Plumb et al., 2002; Wolburg et al., 2003). Indeed, we observed a transient and selective loss of occludin immunoreactivity from the TJ in NMO-like lesions which started $6 \mathrm{~h}$ after lesion induction simultaneous with the observation of the breakdown of the BBB. At that time point occludin immunoreactivity was detected in $31 \%$ of the vessels which decreased further to $3.5 \%$ after $10 \mathrm{~h}$ and $24 \mathrm{~h}$ compared to $93 \%$ occludin positive vessels in control brain tissue. Subsequently occludin expression again increased and $6 \mathrm{~d}$ after lesion induction no alterations in occludin expression were observed compared to uninjected controls (Figure 8). However, although occludin immunoreactivity at the TJ was significantly reduced $24 \mathrm{~h}$ after lesion induction the integrity of the BBB was already restored to molecules $>690$ Da (Figure 5). 
Although occludin, claudin-3 and claudin-5 were suggested to regulate BBB permeability in the CNS, claudin-5 is the only transmembrane molecule that was shown to be crucial for the development of a tight BBB. Also, it was shown to be expressed at significantly higher levels than claudin-1, -3 or -12 in brain EC (Daneman et al., 2010a; Ohtsuki et al., 2007; Ohtsuki et al., 2008). Claudin-5 deficient mice die within $10 \mathrm{~h}$ after birth and show a size selective permeability to tracer molecules smaller than 800 Da (Nitta, 2003). No BBB specific phenotype was detected in occludin or claudin-3 deficient mice (Kooij et al., 2013; Saitou et al., 2000), although the latter showed an impaired brain-CSF-barrier. In addition claudins, but not occludin were shown to induce de novo TJ formation in mouse fibroblasts in transfection studies (Furuse et al., 1998b). Furthermore, overexpression of occludin in Madin-Darby canine kidney II (MDCK II) cells, an epithelial cell line, did not result in a reduced permeability to uncharged molecules although higher TEER values were measured (Van Itallie et al., 2010). However, occludin was suggested to have a regulatory function on TJ tightness. In vitro studies showed that occludin transduces cytokine signaling of tumor necrosis factor (TNF)- $\alpha$ and interferon (INF)- $\nu$ (Van Itallie et al., 2010) and that occludin proteolysis or vascular endothelial growth factor (VEGF) signaling could increase permeability of cell monolayers (Murakami et al., 2009; Wachtel et al., 1999). The experimental data available indicate that the members of the claudin family have key functions in the sealing of the paracellular cleft and the induction of the BBB phenotype. Therefore, in addition to occludin, we also investigated the expression of claudin- 3 and -5 in NMO-like lesions. Indeed, no loss of claudin-3 and -5 immunoreactivity from the TJ was detected at any time point investigated (Figure 8). This indicates that lack of occludin might be compensated for by an intact localization of claudin-3 and -5 at the TJ strands between adjacent EC. This is supported by our observation on the ultrastructural level where we did not observe overt morphological TJ abnormalities or the separation of adjacent EC $6 \mathrm{~h}$ and $24 \mathrm{~h}$ after lesion induction (Figure 9). Furthermore, a rapid increase in mRNA expression of occludin and claudin-5 $10 \mathrm{~h}$ and $24 \mathrm{~h}$ after lesion induction might contribute to the reestablishment of the BBB integrity to soluble tracers (Figure 10).

\subsection{PMN play a crucial role in the breakdown of the BBB in focal NMO like lesions}

In neuroinflammatory diseases like NMO or multiple sclerosis (MS) the development of CNS lesions is accompanied by the infiltration of immune cells and inflammation mediated increases in BBB permeability (Grossman et al., 1986; Lucchinetti et al., 2000; Lucchinetti et 
al., 2002; O'Riordan et al., 1996). To assess whether the immune cell infiltration coincides with the disruption of the BBB in NMO-like lesions a time course experiment was performed. We detected only few CD3 positive T cells in focal NMO-like lesions starting not until $12 \mathrm{~h}$ after lesion induction. Furthermore, it was shown in a model of focal NMO-like lesions in mice that depletion of $\mathrm{T}$ cells does not reduce inflammation, myelin breakdown, or loss of AQP4 immunoreactivity (Saadoun et al., 2011) indicating, in line with our data, that T cells are not required for the development of NMO-like lesions. Contrary to the scarce infiltration of $\mathrm{T}$ cells, the infiltration of abundant numbers of PMN, macrophages and monocytes was observed. $6 \mathrm{~h}$ after lesion induction PMN were the most abundant leukocyte cell type observed at the injection site, and their numbers were significantly higher than these of ED1 positive monocytes/macrophages (Figure 11). Indeed, PMN were shown to be the first cells to infiltrate various tissues in other models of infection or tissue damage, e.g. in models of peritonitis, colitis (Ajuebor et al., 1999; Cailhier et al., 2005; Yamamoto et al., 2008) or ischemia (Barone et al., 1991; Garcia et al., 1994; Matsuo et al., 1994). In our model of NMOlike lesions the activation of the complement cascade after focal injection of NMO-Ab and human complement results in the release of the anaphylatoxin C5a and thus might contribute to the rapid recruitment of immune cells into the brain parenchyma. In addition, infection or tissue damage result in the release of pathogen- and damage-associated molecular pattern molecules (PAMPs and DAMPs), such as lipopolysaccharide (LPS) and nuclear or cytoplasmic components released from necrotic cells. PAMPs and DAMPs are sensed by resident macrophages and dendritic cells which results in their activation. It was shown that there is an early interaction between resident macrophages and PMN in response to DAMPs which might represent nuclear and cytoplasmic components released by necrotic astrocytes in NMO-like lesions. Activated resident macrophages in turn release proinflammatory cytokines (for example interleukine 8 (IL-8) or TNF- $\alpha$ ) and chemokines (e.g. chemokine ( $\mathrm{C}-\mathrm{X}-\mathrm{C}$ motif) ligand 1 (CXCL1), CXCL2 or CXCL5) which attract PMN from the blood into the tissue (Ajuebor et al., 1999; Cailhier et al., 2005; Tian et al., 2007; Yamamoto et al., 2008; Yamasaki et al., 2008; Yanai et al., 2009). PMN, in turn, recruit more PMN and classical monocytes with the release of diverse granule contents (Chertov et al., 1997; Soehnlein et al., 2008) and thereby promote the immune response.

Indeed, PMN were implied to play a role in the development of human and experimental NMO lesions. Infiltration of PMN into the CNS was described in human NMO lesions (Lucchinetti et al., 2002) and was present in all NMO biopsies investigated in the present study (Figure 18). In addition, PMN were described in the CSF of NMO patients (Wingerchuk 
et al., 1999). Using ex vivo spinal cord slice cultures it was demonstrated that neutrophils exacerbate lesion formation in the presence of a sublytic concentration of NMO-Ab in combination with human complement. This exacerbation was associated with the release of the PMN protease NE (Zhang et al., 2011). Later, PMN were implicated to participate in Abdependent cell-mediated cytotoxicity (ADCC) against AQP4 expressing astrocytes. Using in vitro cell culture and ex vivo spinal cord slice cultures it was shown that eosinophils induced cell death of AQP4 positive cells when added to the cultures in combination with recombinant NMO-Ab. When eosinophils were added together with a recombinant NMO-Ab lacking the ADCC effector function, the loss of AQP4 positive cells was significantly reduced (Zhang and Verkman, 2013). The contribution of ADCC mediated astrocyte loss to lesion formation was confirmed in vivo in focal NMO-like lesions and after intravenous (i.v.) injection of NMO-Ab in myelin basic protein (MBP)-preimmunized rats using the same ADCC deficient recombinant NMO-Ab in rats (Wrzos et al., 2014). Furthermore it was suggested that astrocyte targeted ADCC is mediated by the low affinity IgG receptor FcyRIII which is expressed on PMN and macrophages. Indeed, focal injection of NMO-Ab and human complement resulted in significantly smaller lesions in FcpRIII deficient mice compared to wt mice. Likewise, systemic treatment with an anti-FcyRII/III Ab resulted in diminished lesion development, but also in reduced neutrophil blood counts which might contribute together to the reduced pathology that was observed in these mice (Ratelade et al., 2013). In addition, we demonstrated that PMN are crucial for the development of NMO-like lesions. The development of astrocyte depleted lesions was only observed in control animals, but not in animals lacking PMN after treatment with anti-PMN antiserum $6 \mathrm{~h}$ after lesion induction (Figure 12). This is in line with results published by Saadoun and coworkers. They demonstrated that neutropenia induced in mice by injection of rat anti-neutrophil IgG (1A8) resulted in a reduction of astrocyte loss and demyelination. In contrast, induction of neutrophilia by injection of granulocyte colony stimulating factor significantly exacerbated lesion development (Saadoun et al., 2012).

Thus, our findings that PMN are crucial for development of NMO-like lesions are in line with published data in different NMO models. Moreover, we demonstrated that PMN play a central role in the regulation of BBB permeability during their infiltration into the brain parenchyma $6 \mathrm{~h}$ after lesion induction. A strong correlation between the numbers of PMN/lesion site and the extent of FITC-albumin extravasation was detected at this time point (Figure $11 \mathrm{D}$ ). Consequently, depletion of PMN by injection of anti-PMN antiserum prevented - beside the loss of astrocytes - the breakdown of the BBB and the loss of occludin 
from the TJ (Figure 12). In addition, we could show that focal injection of a CNS-unspecific ctrl-Ab together with human complement also results in the infiltration of, although smaller numbers of PMN (Figure 12), coinciding with some leakage of tracer molecules into the parenchyma (Figure 4, Figure 5 and Figure 12) and loss of occludin from few TJ (Figure 8). This emphasizes the importance of PMN in the induction of BBB permeability upon infiltration into the CNS even in the absence of a CNS-specific adaptive immune response. It suggests that in our model, a minimal activation of the complement cascade may result in a local inflammatory response which in turn leads to the recruitment of PMN into the brain. This, however, is substantially amplified by specific Ab-binding after focal injection of NMOAb.

A loss of BBB integrity induced by PMN transmigration is in line with observations in other models of CNS inflammation e.g. in stroke, trauma or experimental autoimmune encephalomyelitis (EAE) (Carlson et al., 2008; Neumann et al., 2015; Schnell et al., 1999). Using 2-photon imaging it was shown that extravasation of PMN into the CNS is associated with a local BBB disruption and infarct formation in an in vivo stroke model (Neumann et al., 2015). Also trauma-induced lesions in the CNS were associated with leukocyte infiltration and increased BBB permeability (Schnell et al., 1999). Furthermore, injection of IL-ß into the brain of juvenile rats or chronic expression of IL-1 in the brain resulted in recruitment of PMN and a subsequent breakdown of the BBB (Bolton et al., 1998; Ferrari et al., 2004). Blockage or deletion of CXCR2, which is important for PMN chemoattraction to the site of inflammation, as well as depletion of $P M N$, resulted in an abrogation of EAE and preservation of the BBB integrity. In this study supplementation of wild type (wt) PMN into CXCR2 deficient mice was sufficient to restore EAE susceptibility and BBB breakdown (Carlson et al., 2008). However, also contrary data is published. It was shown that direct injection of activated PMN into the striatum of mice did not affect BBB integrity (Joice et al., 2009). Additionally, it was demonstrated in vivo and in vitro that transcellular migration of PMN by itself does not necessarily result in the induction of increased permeability (von Wedel-Parlow et al., 2011; Wolburg et al., 2005). These and other studies also demonstrated that in the brain, leukocyte transmigration occurs predominantly by the transcellular pathway and that the TJ appeared unaltered in their ultrastructural morphology after transmigration (Lossinsky et al., 1989; Raine et al., 1990; von Wedel-Parlow et al., 2011; Wolburg et al., 2005). This is in line with our observations in focal NMO-like lesions using EM. An ultrastructural TJ morphology similar to that detected in brain vessels of uninjected control brain was observed in focal NMO-like lesion $6 \mathrm{~h}$ and $24 \mathrm{~h}$ after lesion induction 
(Figure 9). One possibility to explain the increased permeability despite morphologically intact TJ is the published observation that horseradish peroxidase leaks into the brain alongside transmigrating inflammatory cells in vivo (Claudio et al., 1990; Lossinsky et al., 1989) and in vitro (Wong et al., 2007).

In this study we demonstrated that PMN are the first cells to infiltrate the brain parenchyma, that the breakdown of the BBB correlated with PMN extravasation and that depletion of PMN from the blood prevented the loss of BBB integrity $6 \mathrm{~h}$ after lesion induction. However, high numbers of PMN were observed at the injection site $24 \mathrm{~h}$ after lesion induction with a simultaneous restoration of the BBB integrity to vascular tracers $>690 \mathrm{Da}$ (Figure 5). The maximal number of PMN in the lesion was reached between $12 \mathrm{~h}$ and $24 \mathrm{~h}$ after lesion induction with similar numbers of PMN detected around the injection site at both time points. This indicates that infiltration of PMN into the brain parenchyma is completed before $24 \mathrm{~h}$ after lesion induction. Once the infiltration of PMN stops, the BBB might be able to restore its integrity to soluble molecules. Indeed it was shown in vitro that TNF- $\alpha$ mediated PMN transmigration through a brain EC monolayer resulted in induction of permeability to horseradish peroxidase at the transmigration site accompanied by decreased TEER values. However, at the end of the migration period, the EC layer resumed their continuity and integrity to horseradish peroxidase (Wong et al., 2007). In addition, as discussed later in more detail, MMP9 released by PMN might play a role in the breakdown of the BBB. We demonstrated that 20\% of the PMN were immunopositive for MMP9 $6 \mathrm{~h}$ after lesion induction. However, in only $2 \%$ of the PMN cellular MMP9 immunoreactivity was detected $24 \mathrm{~h}$ after lesion induction (Figure 16). PMN release their granule contents early after activation and infiltration. Therefore, infiltrated PMN might represent a lesser activated state at $24 \mathrm{~h}$ compared to $6 \mathrm{~h}$ after lesion induction. Additionally, it has been shown that apoptotic PMN also play a role in the resolution of inflammation. Apoptotic PMN are cleared by macrophages. Upon PMN phagocytosis macrophages produce elevated levels of the antiinflammatory cytokine IL-10 while the production of the proinflammatory cytokines IL-23 and IL-12 decreases (Filardy et al., 2010; Stark et al., 2005). Thereby, macrophages assume a phenotype that promotes tissue repair. In addition, the resolution of inflammation requires the removal of proinflammatory mediators. It has been shown that apoptotic PMN express increased levels of CCR5 which can act as a scavenger receptor for the inflammatory chemokines CCL3 and CCL5 (Ariel et al., 2006). Additionally, PMN might express decoy receptors like the chemokine receptor D6 or IL-1R2 that bind inflammatory cc-chemokines and IL-1, respectively (Bourke et al., 2003; McKimmie et al., 2008). 
However, not only PMN, but also T cells or monocytes were shown to contribute to the disruption of the BBB in other inflammatory models such as EAE (Bartholomaus et al., 2009; Morrissey et al., 1996; Seeldrayers et al., 1993) or after intracerebral injection of chemokine (C-C motif) ligand 2 ( $\mathrm{Ccl} 2)$, an important chemoattracting molecule for monocytes/macrophages (Stamatovic et al., 2005). As mentioned above, a role of T cells can be excluded in our model. However, a possible role of monocytes in NMO-lesion development and in the dysregulation of the BBB cannot be completely ruled out in the study presented here. In addition to PMN (201 \pm 79 cells $/ \mathrm{mm}^{2}$ ), few $(2 \pm 2)$ ED1 (^CD68) positive cells $/ \mathrm{mm}^{2}$, a marker of monocytes/macrophages, were observed in the brain parenchyma $6 \mathrm{~h}$ after lesion induction. Although we demonstrated that depletion of PMN prevented NMO-like lesion formation, the anti-PMN antiserum used here to deplete PMN also resulted in the reduction of peripheral blood cells like monocytes and lymphocytes as investigated by flow cytometry (Figure 13). This is in contrast to a previous study which showed the treatment of Fischer F344 inbred rats with anti-PMN antiserum to result, beside PMN depletion, only in a slight decrease of lymphocytes. The numbers of monocytes in the blood were not investigated (Snipes et al., 1995). Therefore, a possible contribution of monocytes to lesion development requires further investigation. On the one hand an anti-rat neutrophil specific Ab (RP-3) has been described, which we plan to utilize to further specify the role of neutrophils in NMO-like lesion development (Sekiya et al., 1989). On the other hand we plan to specifically address the role of monocytes in lesion development by using CC chemokine receptor type 2 (Ccr2) deficient mice and Ccr2 inhibitors. Ccr2 is expressed on inflammatory monocytes and macrophages and is the receptor for $\mathrm{Ccl}$. It is crucial for monocyte chemotaxis and therefore also for the recruitment of monocytes into the inflamed tissue (Kurihara et al., 1997; Kuziel et al., 1997). Additionally, Ccr2 positive cells have been implied in the disruption of the BBB (Stamatovic et al., 2005). For this purpose NMO-like lesions in mice are currently established in our laboratory.

\subsection{PMN attracting molecules and released granule contents modulate NMO- like lesion development and BBB permeability}

The development of focal NMO-like lesions was shown to be complement-dependent. After injection of NMO-Ab and human complement, lesion development is triggered by the binding of the NMO-Ab to AQP4 on astrocytes and the subsequent activation of the complement cascade which results in astrocyte lysis (Wrzos et al., 2014). Furthermore, activated complement deposits were described both in experimental and human NMO 
lesions (Bennett et al., 2009; Lucchinetti et al., 2002) and antibodies defective in complement-dependent cytotoxicity $(C D C)$ diminish the formation of NMO-like lesions (Ratelade et al., 2013; Tradtrantip et al., 2012; Wrzos et al., 2014). After activation of the complement cascade, C5 convertase becomes activated and cleaves the inactive C5 into the active component $\mathrm{C} 5 \mathrm{~b}$ and the anaphylatoxin $\mathrm{C} 5 \mathrm{a}$. Inhibition of this step by using the monoclonal antibody eculizumab, directed against C5, inhibits the effector pathway of the complement cascade, and significantly reduced attack frequency and improved or stabilized the neurological disability measures in NMO patients (Pittock et al., 2013). Further downstream, activation of the complement cascade results in the formation of the final membrane attack complex resulting in astrocyte lysis. Furthermore, the anaphylatoxin $\mathrm{C} 5 \mathrm{a}$ is released. Two receptors of C5a are known: the C5a receptor-like 2 (C5L2) and the seven transmembrane-containing $\mathrm{G}$ protein-coupled receptor $\mathrm{C} 5 \mathrm{aR}$. C5a was shown to be a potent peptide mediator of inflammation with $\mathrm{C} 5 \mathrm{aR}$ being widely expressed on different cell types. In the CNS C5aR is, e.g., expressed by astrocytes, microglia, oligodendrocytes and EC (Gasque et al., 1995; Lacy et al., 1995; Laudes et al., 2002; Nataf et al., 2001) and can induce, e.g., the expression of adhesion molecules on the endothelial surface (Albrecht et al., 2004). Moreover, C5aR is expressed on different immune cells, and C5a acts as an important chemoattractant for PMN, macrophages and monocytes (Chenoweth et al., 1982; Chenoweth and Hugli, 1978).

Therefore, we addressed the question whether the development of NMO-like lesions can be attenuated by systemic treatment of animals with the small molecule C5aR antagonist PMX53 (Finch et al., 1999; Wong et al., 1998), which has been shown to be effective e.g. in the treatment of rheumatoid arthritis in rats (Woodruff et al., 2002). Indeed, application of the C5a-antagonist $3 \mathrm{~h}$ after lesion induction significantly decreased the development of astrocyte depletion lesions after $6 \mathrm{~h}$. However, neither the number of infiltrated PMN nor the BBB permeability in the lesions was significantly altered after treatment with PMX-53 (Figure 14). These apparently conflicting data might be explained by the complex functions C5aR signaling triggers in PMN. Additionally to the function of C5a as a chemoattractant, binding of $\mathrm{C} 5 \mathrm{a}$ to $\mathrm{C} 5 \mathrm{aR}$ is also implied in the full activation of PMN which is assumed to be a two-step process. The first step, also called priming, is mediated by pro-inflammatory cytokines such as TNF- $\alpha$ or IL-1 or by the contact with the activated endothelium (Fuortes et al., 1993; Lloyds et al., 1995; Nathan et al., 1989; Yagisawa et al., 1995). Full activation can be triggered upon exposure to PAMPs, growth factors or chemoattractants like C5a. Binding of $\mathrm{C} 5 \mathrm{a}$ to the $\mathrm{C} 5 \mathrm{aR}$ on PMN mediates enhanced expression of adhesion molecules, 
chemotaxis, release of granule enzymes, oxidative burst, phagocytosis and delayed apoptosis (Becker et al., 1985; Foreman et al., 1994; Goldstein et al., 1975; Kernen et al., 1991; Mollnes et al., 2002; Perianayagam et al., 2004; Snyderman and Pike, 1984).

During lesion initiation the complement mediated lysis of astrocytes may result in the development of an inflammatory milieu which results in the activation of the endothelium, in which also C5a has been implied. Endothelial cells were also shown to express C5aR and activation of $\mathrm{C} 5 \mathrm{aR}$ signaling pathways were demonstrated to result in the expression of cytokines, chemokines and adhesion molecules on the endothelial surface (Albrecht et al., 2004). Therefore, the contact of PMN to the activated endothelium at the injection side may lead to the priming of PMN and transmigration. This sequence of events is supported by observations made by Vincent and coworkers. They demonstrated that NMO-IgG binding to astrocytes and subsequent CDC mediated astrocyte lysis resulted in the attraction of PMN through an EC monolayer in vitro (Vincent et al., 2008). However, once the BBB is overcome PMN of animals treated with C5aR antagonist might fail to become fully activated (Becker et al., 1985; Foreman et al., 1994; Goldstein et al., 1975; Kernen et al., 1991; Mollnes et al., 2002; Perianayagam et al., 2004; Snyderman and Pike, 1984). This might result in reduced release of granule contents, reduced phagocytosis and reduced participation in ADCC which might result in the reduced loss of astrocyte observed after treatment with the C5aR antagonist although the BBB permeability and the number of infiltrated PMN are not significantly altered. However, additional in vitro experiments are planned to further investigate this hypothesis. The effect of $\mathrm{C} 5 \mathrm{a}$ incubation in the absence and presence of activated endothelial cells on the activation of PMN will be determined and subsequently their ability to lyse astrocytes in an ADCC depended manner together with NMO-Ab will be measured. In addition, the effect of $\mathrm{C} 5 \mathrm{aR}$ antagonist treatment will be investigated in this system.

To address the question how PMN mediate the breakdown of the $B B B$, we focused on proteases released from PMN granules upon activation, which have been reported to contribute to the induction of BBB permeability. During the granulopoiesis in the bone marrow PMN granules are formed successively. Four types of granules are known: primary (azurophilic), secondary (specific), tertiary (gelatinase) and secretory granules. Granules contain different sets of proteases, receptors, adhesion molecules and antibacterial proteins. The degree of PMN activation is important for the release of specific granule subtypes. In a first step secretory granules are released (Borregaard et al., 1990), containing 
primarily receptors and adhesion molecules (e.g. the $ß_{2}$-integrin MAC-1, CD14 and complement receptor 1 ). Upon fusion of the secretory vesicle membrane with the cell membrane these molecules are expressed on the cell surface and enhance firm adhesion to the endothelium and the chemotaxis-directed migration (Borregaard et al., 1990; Detmers et al., 1995; Miller et al., 1987; Sengeløv et al., 1994). Subsequently, tertiary and secondary granules are released. They contain e.g. the proteases MMP9 and collagenase which degrade the basement membrane and extracellular matrix and thereby facilitate PMN egress and migration (Dewald et al., 1982; Kjeldsen et al., 1993; Murphy et al., 1980). Primary granules are mobilized at the end and thereby release proteins that are thought to participate in bactericidal activity and contain, e.g. myeloperoxidase, NE and cathepsin G (Cramer et al., 1985; Malemud and Janoff, 1975; Ohlsson and Odsson, 1974).

MMP9, cathepsin G and NE are PMN proteases that are implied in disruption of the BBB (Chen et al., 2009; Hermant et al., 2003; Suzuki et al., 1994). Therefore, we investigated the effect of these proteases on the electrical resistance of RBEC in vitro. No alterations in TEER values were observed after incubation of RBEC monolayers with either cathepsin G or NE. However, treatment of RBEC with MMP9 resulted in a reduction of TEER values by $49.8 \%$ compared to untreated controls (Figure $15 \mathrm{~B}$ ). This observation was not due to RBEC death, which in turn would result in the disruption of the RBEC monolayer and thereby decrease TEER values (Figure $15 \mathrm{C}$ ). A possible role of MMP9 released from PMN in the breakdown of the BBB has been discussed in the literature. Especially in models of stroke and traumatic brain injury, MMP9 may play a crucial role in the disruption of the BBB. Elevated MMP9 levels are observed in stroke patients and correlate with poor neurologic outcome (Castellanos et al., 2003; Horstmann et al., 2003; Montaner et al., 2003). In autopsy tissue, MMP9 was mainly detected around vessels and associated with neutrophil infiltration (Rosell et al., 2008; Rosell et al., 2006). In experimental models of focal ischemia elevated MMP9 levels were demonstrated, which were associated with loss of BBB integrity, hemorrhage and neuronal death (Romanic et al., 1998). In MMP9 deficient mice or upon pharmacologic MMP inhibition subsequent to induction of focal cerebral ischemia smaller lesions and protection of the BBB integrity were described (Asahi et al., 2000; Asahi et al., 2001; Cui et al., 2012; Gu et al., 2005; Jiang et al., 2001; Koistinaho et al., 2005; Romanic et al., 1998; Rosenberg et al., 1998). Comparable findings were observed after traumatic brain injury in humans and experimental models (Hadass et al., 2013; Muradashvili et al., 2015; Suehiro et al., 2004). 
In addition, MMP9 has been implied to play a role in MS. Elevated MMP9 levels were measured in the CSF of MS patients (Gijbels et al., 1992; Hosokawa et al., 2011; Mandler et al., 2001) and in the serum and CNS tissue during EAE (Clements et al., 1997; Kandagaddala et al., 2012; Kieseier et al., 1998; Nygårdas and Hinkkanen, 2002). Furthermore, young, 3-4 week-old MMP9 deficient mice were shown to be less susceptible to EAE compared to agematched wt mice. In adult mice, however, no difference in the development and course of EAE was observed (Dubois et al., 1999). However, mice deficient in both MMP9 and MMP2 are resistant to EAE (Agrawal et al., 2006), and also pharmacologic inhibition of MMPs ameliorated EAE (Gijbels et al., 1994; Hewson et al., 1995; Niimi et al., 2013).

Limited data regarding the role of MMP9 in NMO is available. Like in MS the MMP9 CSF levels in NMO patients were shown to be elevated. Whether MMP9 levels in the CSF of NMO patients differ from those of MS patients is controversial: while one group reported significantly higher MMP9 levels in the CSF of NMO patients (Mandler et al., 2001), another group did not detect any differences (Hosokawa et al., 2011). However the latter showed that serum MMP9 concentrations were significantly higher in NMO than in MS patients and correlated with expanded disability status scale scores (EDSS) and BBB disruption, as measured by CSF:serum albumin ratios (Hosokawa et al., 2011). Utilizing our model of focal NMO-like lesions we could show that $20 \%$ of the infiltrating PMN at the injection site were immunopositive for MMP9 $6 \mathrm{~h}$ after lesion induction. At subsequent time points the percentage of MMP9 positive PMN decreased. Only 2\% of PMN were immunopositive for MMP9 $24 \mathrm{~h}$ after lesion induction, and no MMP9 reactivity was detected on day 3 after lesion induction (Figure 16), indicating an early release of MMP9 by PMN. Since treatment of RBEC with MMP9 induced a reduction of TEER values in vitro and MMP9 positive PMN were only found in early lesions in vivo, MMP9 released by PMN may be an important mediator of BBB disruption in NMO-like lesions. However, to investigate the functional effects of MMP9 on BBB integrity and lesion development in focal NMO-like lesions, further experiments are necessary. Studies are planned to assess the effect of pharmacologic inhibition of MMPs by using the specific MMP2/MMP9 inhibitor SB-3CT. In addition, we will investigate the development of NMO-like lesions and the breakdown of BBB in MMP9 deficient mice.

However, the mechanism by which MMP9 regulates the BBB permeability is still unknown. One proposed mechanism is the cleavage of the TJ molecule occludin by MMP9 (Bojarski, 2004; Lischper et al., 2010; Liu et al., 2009; Reijerkerk et al., 2006; Yang et al., 2007). Indeed, a transient loss of occludin immunoreactivity from the TJ of brain vessels was observed after 
lesion induction. After $6 \mathrm{~h}$, loss of occludin immunoreactivity from about $70 \%$ of the vessels coincided with infiltration of PMN into the lesions (Figure 8). However, direct incubation of RBEC for $2 \mathrm{~h}$ with active MMP9 did not result in loss of occludin immunoreactivity from the cell-cell contacts although a decrease in TEER values was observed (Figure 15 B, D). To further investigate a possible fragmentation of occludin after MMP9 treatment western blot analyses will be performed.

As mentioned before NE did not show a direct effect on TEER values of RBEC in vitro (Figure 15). However, published data suggests that NE released by PMN might play a role in the breakdown of the BBB and in the development of NMO-like lesion in vivo (Saadoun et al., 2012). To investigate the role of NE in the development of focal NMO-like lesions, the competitive NE inhibitor Sivelestat was used. Sivelestat was developed to inhibit human NE, but is also effective in other species like rat, rabbit and mouse (Kawabata et al., 1991). Indeed, systemic treatment of rats with Sivelestat $3 \mathrm{~h}$ after lesion induction resulted in significantly smaller astrocyte depleted lesions compared to vehicle treated controls after $6 \mathrm{~h}$ (Figure 17). These results are in line with published data. Zhang and colleagues have shown in an ex vivo NMO-model, that incubation of spinal cord slice cultures with neutrophils and an insufficient concentration of complement and NMO-Ab resulted in exacerbated lesion formation which was significantly reduced after treatment with Sivelestat. Conversely, supplementation of NE potentiated lesion development in the absence of neutrophils (Zhang et al., 2011). In vivo it was demonstrated in mice that intracerebral injection of NMO-Ab and human complement together with Sivelestat and a cathepsin G inhibitor reduced lesion size. Similar results were obtained after systemic treatment with Sivelestat. In addition, significantly fewer extravasated neutrophils were observed within the lesion. (Saadoun et al., 2012).

Fewer extravasating PMN were also observed in the brain parenchyma in our model after treatment with Sivelestat. Surprisingly, this did not result in a decreased permeability of the BBB to the vascular tracer FITC-albumin compared to vehicle treated animals (Figure 17). Whether NE directly increases the permeability of the BBB is conflicting in the literature. In vitro, incubation of EC with activated neutrophils or purified NE was shown to increase permeability (Hermant et al., 2003). Furthermore, NE mediated increase of endothelial permeability is prevented by Sivelestat (Ikegame et al., 2010; Suzuki et al., 1994). In contrast, removal of NE from the secreted supernatant of PMN by immunoabsorption did not result in different TEER values compared to NE-containing supernatant (Gautam et al., 1998; Gautam 
et al., 2000). In vivo, in a rat model of spinal cord injury and in a mouse model of focal ischemia, treatment with Sivelestat significantly reduced neurological deficits, neutrophil accumulation and BBB disruption (Ikegame et al., 2010; Tonai et al., 2001). However, using NE knockout mice it was demonstrated that deficiency of NE exacerbated pathology after ventilator-induced lung injury. This was surprising since NE deficient mice showed also lower numbers of neutrophils in the alveolar spaces. One possible explanation suggested by the authors is the finding that the intercellular adhesion molecule 1 (ICAM-1) is a substrate of NE (Champagne et al., 1998; Kaynar et al., 2008). By cleavage of ICAM-1 PMN adhesion to the endothelium is terminated and PMN can transmigrate into the tissue. However, in the absence of NE the interaction of PMN with the endothelium might be prolonged which leads to an increased permeability (Kaynar et al., 2008). This finding gives a possible explanation for the discrepancy we observed after treatment with Sivelestat in focal NMO-like lesions which resulted in smaller astrocyte depleted lesions and less infiltrating PMN, but no difference in BBB permeability (Figure 17). Upon focal injection of NMO-Ab and human complement the endothelium becomes activated which may be associated with an increased expression of ICAM-1 on the luminal side of the vasculature. This in turn leads to ICAM-1 mediated adherence of PMN to the endothelium, which may be prolonged in animals treated with Sivelestat, resulting also in reduced numbers of transmigrated cells. This explanation is supported by observations made by Saadoun and colleagues. They observed that treatment with Sivelestat did not inhibit binding of neutrophils to the endothelium, but their extravasation into the brain parenchyma (Saadoun et al., 2012). Prolonged contact of activated PMN with endothelial cells was shown to result in cell injury (Kaynar et al., 2008) and might therefore induce increases in BBB permeability at the sites of PMN adherence resulting in similar tracer extravasation in Sivelestat and vehicle treated animals. 


\section{$5 \quad$ SUMMARY AND CONCLUSIONS}

Neuromyelitis optica (NMO) is a demyelinating autoimmune disease of the central nervous system. Astrocytes were shown to be the major target in this disease, and autoantibodies against aquaporin4 (AQP4) are detected in about 70\% of NMO patients. The binding of NMO-antibodies (Ab) to AQP4, a water channel which is highly expressed on astrocytic endfeet, results in complement- and cell-mediated depletion of astrocytes in acute lesions. The breakdown of the blood-brain barrier (BBB) is an early and prominent event in NMO as evidenced by gadolinium enhanced magnetic resonance imaging (MRI), but the structural and molecular correlates of the BBB disruption are not yet well understood. We addressed this question in a focal NMO model in the rat, which is based on the intracerebral injection of a human recombinant NMO-Ab directed against AQP4 together with human complement. These lesions mimic key features of early human NMO lesions, including loss of astrocytes and oligodendrocytes, demyelination and infiltration of immune cells.

The development, maintenance and repair of the BBB were shown to be dependent on pericytes and astrocytes that closely interact with endothelial cells (EC). Therefore we hypothesized that the NMO-Ab mediated depletion of astrocytes in NMO-like lesions might contribute to the disruption of the BBB. To investigate this hypothesis, time course experiments were performed, which demonstrated that the onset of astrocyte lesion development coincided with the breakdown of the BBB $6 \mathrm{~h}$ after lesion induction. However, the integrity of the BBB to vascular tracers with molecular sizes of $>690$ Da was restored already after $24 \mathrm{~h}$, at least $2 \mathrm{~d}$ before astrocytes repopulated the lesion. Moreover, in a transwell co-culture model of the BBB we did not detect alterations in the transendothelial resistance of EC monolayers when cultured with or without astrocytes. No loss of pericytes from astrocyte depleted lesions was observed. Thus, pericytes might contribute to the restoration of the BBB in the absence of astrocytes.

Furthermore, a detailed analysis of the tight junctions (TJ), which restrict the paracellular diffusion of solutes across the BBB, was performed. To investigate if the loss of BBB integrity is associated with TJ disruption the expression of occludin, claudin- 3 and -5 at the TJ was assessed. A transient loss of occludin was detected in NMO-like lesions starting $6 \mathrm{~h}$ after lesion induction. The expression of claudin-3 and -5 was not altered. Interestingly, the BBB integrity to vascular tracer molecules was re-established in the absence of occludin $24 \mathrm{~h}$ after lesion induction. Moreover, no morphological alterations of the TJ structure were observed on the ultrastructural level. Claudin-5, but not occludin or claudin-3, was shown to 
be crucial for sealing the paracellular cleft which may in part explain these observations. A rapid increase of occludin and claudin-5 mRNA expression was found $10 \mathrm{~h}$ and $24 \mathrm{~h}$ after lesion induction which might contribute to the re-establishment of the BBB integrity to solutes.

In models of neuroinflammation it has been demonstrated that infiltrating immune cells contribute to the induction of BBB permeability. We found that $6 \mathrm{~h}$ after lesion induction polymorphonuclear cells (PMN) were the most abundant infiltrating leukocytes. At that time point, the number of PMN in the lesions correlated with the extent of FITC-albumin extravasation. Consequently, systemic depletion of PMN prevented breakdown of the BBB and loss of occludin from the TJ. Interestingly, also astrocyte lesion formation was completely abrogated. Since PMN appear to be crucial for astrocyte lesion development we hypothesized that the complement component $\mathrm{C} 5 \mathrm{a}$ receptor ( $\mathrm{C} 5 \mathrm{aR})$, which is important for PMN attraction and activation, may be important for lesion development. Indeed, systemic treatment of rats with the $\mathrm{C} 5 \mathrm{aR}$ antagonist PMX-53 reduced the area of astrocyte loss in NMO-like lesions. Additionally, it has been shown that proteases released from PMN granules upon activation were involved in the regulation of BBB permeability. We could demonstrate that treatment of rat brain EC monolayers with the protease MMP9 decreased their electrical resistance in vitro. Also, we detected MMP9 in early infiltrating PMN in focal NMO-like lesions after $6 \mathrm{~h}$ and $12 \mathrm{~h}$. Furthermore, systemic inhibition of the neutrophil elastase using Sivelestat reduced the area of astrocyte loss and the numbers of infiltrating $\mathrm{PMN}$ in vivo. Ultimately, we showed that infiltrating PMN are a component of the inflammatory infiltrate in early human NMO lesions.

To conclude, this thesis shows a crucial involvement of PMN in the development of NMOlike lesions. Our data indicate that infiltrating PMN mediate the breakdown of the BBB $6 \mathrm{~h}$ after lesion induction. This increase in permeability allows complement factors and immune cells to gain access to the CNS and to contribute to complement- and cell-mediated depletion of astrocytes. These findings together with the detection of PMN in early human NMO lesions identify infiltrating PMN and the concomitant breakdown of the BBB as promising targets for future therapeutic approaches. 


\section{BIBLIOGRAPHY}

Abadier, M., Haghayegh Jahromi, N., Cardoso Alves, L., Boscacci, R., Vestweber, D., Barnum, S., Deutsch, U., Engelhardt, B., and Lyck, R. (2015). Cell surface levels of endothelial ICAM-1 influence the transcellular or paracellular T-cell diapedesis across the blood-brain barrier. Eur J Immunol 45, 1043-1058.

Abbott, N.J., Patabendige, A.A., Dolman, D.E., Yusof, S.R., and Begley, D.J. (2010). Structure and function of the blood-brain barrier. Neurobiology of Disease 37, 13-25.

Abbott, N.J., Rönnbäck, L., and Hansson, E. (2006). Astrocyte-endothelial interactions at the bloodbrain barrier. Nature Reviews Neuroscience 7, 41-53.

Adair, J.C., Charlie, J., Dencoff, J.E., Kaye, J.A., Quinn, J.F., Camicioli, R.M., Stetler-Stevenson, W.G., and Rosenberg, G.A. (2004). Measurement of Gelatinase B (MMP-9) in the Cerebrospinal Fluid of Patients With Vascular Dementia and Alzheimer Disease. Stroke 35, e159-e162.

Agrawal, S., Anderson, P., Durbeej, M., van Rooijen, N., Ivars, F., Opdenakker, G., and Sorokin, L.M. (2006). Dystroglycan is selectively cleaved at the parenchymal basement membrane at sites of leukocyte extravasation in experimental autoimmune encephalomyelitis. J Exp Med 203, 1007-1019.

Ajuebor, M.N., Das, A.M., Virag, L., Flower, R.J., Szabo, C., and Perretti, M. (1999). Role of resident peritoneal macrophages and mast cells in chemokine production and neutrophil migration in acute inflammation: evidence for an inhibitory loop involving endogenous IL-10. J Immunol 162, 16851691.

Albrecht, E.A., Chinnaiyan, A.M., Varambally, S., Kumar-Sinha, C., Barrette, T.R., Sarma, J.V., and Ward, P.A. (2004). C5a-induced gene expression in human umbilical vein endothelial cells. Am J Pathol 164, 849-859.

Alexander, J.S., Harris, M.K., Wells, S.R., Mills, G., Chalamidas, K., Ganta, V.C., McGee, J., Jennings, M.H., Gonzalez-Toledo, E., and Minagar, A. (2010). Alterations in serum MMP-8, MMP-9, IL-12p40 and IL-23 in multiple sclerosis patients treated with interferon-beta1b. Mult Scler 16, 801-809.

Almekhlafi, M.A., Clark, A.W., Lucchinetti, C.F., Zhang, Y., Power, C., and Bell, R.B. (2011). Neuromyelitis optica with extensive active brain involvement: an autopsy study. Arch Neurol 68, 508512.

Alon, R., Kassner, P.D., Carr, M.W., Finger, E.B., Hemler, M.E., and Springer, T.A. (1995). The integrin VLA-4 supports tethering and rolling in flow on VCAM-1. J Cell Biol 128, 1243-1253.

Alvarez, J.I., Dodelet-Devillers, A., Kebir, H., Ifergan, I., Fabre, P.J., Terouz, S., Sabbagh, M., Wosik, K., Bourbonniere, L., Bernard, M., et al. (2011). The Hedgehog pathway promotes blood-brain barrier integrity and CNS immune quiescence. Science 334, 1727-1731.

Ando-Akatsuka, Y., Saitou, M., Hirase, T., Kishi, M., Sakakibara, A., Itoh, M., Yonemura, S., Furuse, M., and Tsukita, S. (1996). Interspecies diversity of the occludin sequence: cDNA cloning of human, mouse, dog, and rat-kangaroo homologues. J Cell Biol 133, 43-47.

Argaw, A.T., Asp, L., Zhang, J., Navrazhina, K., Pham, T., Mariani, J.N., Mahase, S., Dutta, D.J., Seto, J., Kramer, E.G., et al. (2012). Astrocyte-derived VEGF-A drives blood-brain barrier disruption in CNS inflammatory disease. J Clin Invest 122, 2454-2468.

Argaw, A.T., Gurfein, B.T., Zhang, Y., Zameer, A., and John, G.R. (2009). VEGF-mediated disruption of endothelial CLN-5 promotes blood-brain barrier breakdown. Proc Natl Acad Sci U S A 106, 1977-1982. 
Ariel, A., Fredman, G., Sun, Y.P., Kantarci, A., Van Dyke, T.E., Luster, A.D., and Serhan, C.N. (2006). Apoptotic neutrophils and $T$ cells sequester chemokines during immune response resolution through modulation of CCR5 expression. Nat Immunol 7, 1209-1216.

Armulik, A., Genove, G., Mae, M., Nisancioglu, M.H., Wallgard, E., Niaudet, C., He, L., Norlin, J., Lindblom, P., Strittmatter, K., et al. (2010). Pericytes regulate the blood-brain barrier. Nature 468, 557-561.

Asahi, M., Asahi, K., Jung, J.C., del Zoppo, G.J., Fini, M.E., and Lo, E.H. (2000). Role for matrix metalloproteinase 9 after focal cerebral ischemia: effects of gene knockout and enzyme inhibition with BB-94. J Cereb Blood Flow Metab 20, 1681-1689.

Asahi, M., Wang, X., Mori, T., Sumii, T., Jung, J.C., Moskowitz, M.A., Fini, M.E., and Lo, E.H. (2001). Effects of matrix metalloproteinase- 9 gene knock-out on the proteolysis of blood-brain barrier and white matter components after cerebral ischemia. J Neurosci 21, 7724-7732.

Asgari, N., Lillevang, S.T., Skejoe, H.P., Falah, M., Stenager, E., and Kyvik, K.O. (2011). A populationbased study of neuromyelitis optica in Caucasians. Neurology 76, 1589-1595.

Aurrand-Lions, M., Johnson-Leger, C., Wong, C., Du Pasquier, L., and Imhof, B.A. (2001). Heterogeneity of endothelial junctions is reflected by differential expression and specific subcellular localization of the three JAM family members. Blood 98, 3699-3707.

Balda, M.S., Garrett, M.D., and Matter, K. (2003). The ZO-1-associated Y-box factor ZONAB regulates epithelial cell proliferation and cell density. The Journal of Cell Biology 160, 423-432.

Balda, M.S., Whitney, J.A., Flores, C., Gonzalez, S., Cereijido, M., and Matter, K. (1996). Functional dissociation of paracellular permeability and transepithelial electrical resistance and disruption of the apical-basolateral intramembrane diffusion barrier by expression of a mutant tight junction membrane protein. J Cell Biol 134, 1031-1049.

Bar, T. (1983). Patterns of vascularization in the developing cerebral cortex. Ciba Found Symp 100, 20-36.

Barone, F.C., Hillegass, L.M., Price, W.J., White, R.F., Lee, E.V., Feuerstein, G.Z., Sarau, H.M., Clark, R.K., and Griswold, D.E. (1991). Polymorphonuclear leukocyte infiltration into cerebral focal ischemic tissue: myeloperoxidase activity assay and histologic verification. J Neurosci Res 29, 336-345.

Bartholomaus, I., Kawakami, N., Odoardi, F., Schlager, C., Miljkovic, D., Ellwart, J.W., Klinkert, W.E.F., Flugel-Koch, C., Issekutz, T.B., Wekerle, H., et al. (2009). Effector T cell interactions with meningeal vascular structures in nascent autoimmune CNS lesions. Nature 462, 94-98.

Becker, E.L., Kermode, J.C., Naccache, P.H., Yassin, R., Marsh, M.L., Munoz, J.J., and Sha'afi, R.I. (1985). The inhibition of neutrophil granule enzyme secretion and chemotaxis by pertussis toxin. J Cell Biol 100, 1641-1646.

Bell, R.D., Winkler, E.A., Sagare, A.P., Singh, I., LaRue, B., Deane, R., and Zlokovic, B.V. (2010). Pericytes control key neurovascular functions and neuronal phenotype in the adult brain and during brain aging. Neuron 68, 409-427.

Bennett, J., Lam, C., Kalluri, S., Saikali, P., Bautista, K., Dupree, C., Glogowska, M., Case, D., Antel, J., Owens, G., et al. (2009). Intrathecal pathogenic anti-aquaporin-4 antibodies in early neuromyelitis optica. Ann Neurol 66, 617 - 629. 
Berezowski, V., Landry, C., Dehouck, M.P., Cecchelli, R., and Fenart, L. (2004). Contribution of glial cells and pericytes to the mRNA profiles of P-glycoprotein and multidrug resistance-associated proteins in an in vitro model of the blood-brain barrier. Brain Res 1018, 1-9.

Berlin, C., Bargatze, R.F., Campbell, J.J., von Andrian, U.H., Szabo, M.C., Hasslen, S.R., Nelson, R.D., Berg, E.L., Erlandsen, S.L., and Butcher, E.C. (1995). $\alpha 4$ integrins mediate lymphocyte attachment and rolling under physiologic flow. Cell 80, 413-422.

Bernal, F., Elias, B., Hartung, H.P., and Kieseier, B.C. (2009). Regulation of matrix metalloproteinases and their inhibitors by interferon-beta: a longitudinal study in multiple sclerosis patients. Mult Scler 15, 721-727.

Bernardes-Silva, M., Anthony, D.C., Issekutz, A.C., and Perry, V.H. (2001). Recruitment of neutrophils across the blood-brain barrier: the role of E- and P-selectins. J Cereb Blood Flow Metab 21, 11151124.

Bichuetti, D.B., Rivero, R.L., Oliveira, D.M., Souza, N.A., Abdala, N., Oliveira, E.M., and Gabbai, A.A. (2008). Neuromyelitis optica: brain abnormalities in a Brazilian cohort. Arq Neuropsiquiatr 66, 1-4.

Bjarnegård, M., Enge, M., Norlin, J., Gustafsdottir, S., Fredriksson, S., Abramsson, A., Takemoto, M., Gustafsson, E., Fässler, R., and Betsholtz, C. (2004). Endothelium-specific ablation of PDGFB leads to pericyte loss and glomerular, cardiac and placental abnormalities. Development 131, 1847-1857.

Blum, M.S., Toninelli, E., Anderson, J.M., Balda, M.S., Zhou, J., O'Donnell, L., Pardi, R., and Bender, J.R. (1997). Cytoskeletal rearrangement mediates human microvascular endothelial tight junction modulation by cytokines, Vol 273.

Boado, R.J., Li, J.Y., Nagaya, M., Zhang, C., and Pardridge, W.M. (1999). Selective expression of the large neutral amino acid transporter at the blood-brain barrier. Proc Natl Acad Sci U S A 96, 1207912084.

Bojarski, C. (2004). The specific fates of tight junction proteins in apoptotic epithelial cells. Journal of Cell Science 117, 2097-2107.

Bolton, S.J., Anthony, D.C., and Perry, V.H. (1998). Loss of the tight junction proteins occludin and zonula occludens-1 from cerebral vascular endothelium during neutrophil-induced blood-brain barrier breakdown in vivo. Neuroscience 86, 1245-1257.

Bonnan, M., Valentino, R., Olindo, S., Mehdaoui, H., Smadja, D., and Cabre, P. (2009). Plasma exchange in severe spinal attacks associated with neuromyelitis optica spectrum disorder. Multiple Sclerosis 15, 487-492.

Borowsky, B., Mezey, E., and Hoffman, B.J. (1993). Two glycine transporter variants with distinct localization in the CNS and peripheral tissues are encoded by a common gene. Neuron 10, 851-863.

Borregaard, N., Christensen, L., Bejerrum, O.W., Birgens, H.S., and Clemmensen, I. (1990). Identification of a highly mobilizable subset of human neutrophil intracellular vesicles that contains tetranectin and latent alkaline phosphatase. J Clin Invest 85, 408-416.

Bourke, E., Cassetti, A., Villa, A., Fadlon, E., Colotta, F., and Mantovani, A. (2003). IL-1 beta scavenging by the type II IL-1 decoy receptor in human neutrophils. J Immunol 170, 5999-6005.

Bradl, M., Misu, T., Takahashi, T., Watanabe, M., Mader, S., Reindl, M., Adzemovic, M., Bauer, J., Berger, T., Fujihara, K., et al. (2009). Neuromyelitis optica: Pathogenicity of patient immunoglobulin in vivo. Annals of Neurology 66, 630-643. 
Brown, H., Hien, T.T., Day, N., Mai, N.T., Chuong, L.V., Chau, T.T., Loc, P.P., Phu, N.H., Bethell, D., Farrar, J., et al. (1999). Evidence of blood-brain barrier dysfunction in human cerebral malaria. Neuropathol Appl Neurobiol 25, 331-340.

Bush, T.G., Puvanachandra, N., Horner, C.H., Polito, A., Ostenfeld, T., Svendsen, C.N., Mucke, L., Johnson, M.H., and Sofroniew, M.V. (1999). Leukocyte Infiltration, Neuronal Degeneration, and Neurite Outgrowth after Ablation of Scar-Forming, Reactive Astrocytes in Adult Transgenic Mice. Neuron 23, 297-308.

Butt, A.M., Jones, H.C., and Abbott, N.J. (1990). Electrical resistance across the blood-brain barrier in anaesthetized rats: a developmental study. J Physiol 429, 47-62.

Cabre, P., Heinzlef, O., Merle, H., Buisson, G.G., Bera, O., Bellance, R., Vernant, J.C., and Smadja, D. (2001). MS and neuromyelitis optica in Martinique (French West Indies). Neurology 56, 507-514.

Cabrera-Gomez, J.A., Kurtzke, J.F., Gonzalez-Quevedo, A., and Lara-Rodriguez, R. (2009). An epidemiological study of neuromyelitis optica in Cuba. J Neurol 256, 35-44.

Cabrera-Gomez, J.A., Quevedo-Sotolongo, L., Gonzalez-Quevedo, A., Lima, S., Real-Gonzalez, Y., Cristofol-Corominas, M., Romero-Garcia, K., Ugarte-Sanchez, C., Jordan-Gonzalez, J., de la Nuez, J.E., et al. (2007). Brain magnetic resonance imaging findings in relapsing neuromyelitis optica. Mult Scler 13, 186-192.

Cailhier, J.F., Partolina, M., Vuthoori, S., Wu, S., Ko, K., Watson, S., Savill, J., Hughes, J., and Lang, R.A. (2005). Conditional macrophage ablation demonstrates that resident macrophages initiate acute peritoneal inflammation. J Immunol 174, 2336-2342.

Carlson, T., Kroenke, M., Rao, P., Lane, T.E., and Segal, B. (2008). The Th17-ELR+ CXC chemokine pathway is essential for the development of central nervous system autoimmune disease. Journal of Experimental Medicine 205, 811-823.

Carman, C.V., and Springer, T.A. (2004). A transmigratory cup in leukocyte diapedesis both through individual vascular endothelial cells and between them. J Cell Biol 167, 377-388.

Carmeliet, P., Ferreira, V., Breier, G., Pollefeyt, S., Kieckens, L., Gertsenstein, M., Fahrig, M., Vandenhoeck, A., Harpal, K., Eberhardt, C., et al. (1996). Abnormal blood vessel development and lethality in embryos lacking a single VEGF allele. Nature 380, 435-439.

Carvalho-Tavares, J., Hickey, M.J., Hutchison, J., Michaud, J., Sutcliffe, I.T., and Kubes, P. (2000). A Role for Platelets and Endothelial Selectins in Tumor Necrosis Factor- $\alpha-I n d u c e d$ Leukocyte Recruitment in the Brain Microvasculature. Circulation Research 87, 1141-1148.

Castellanos, M., Leira, R., Serena, J., Pumar, J.M., Lizasoain, I., Castillo, J., and Dávalos, A. (2003). Plasma Metalloproteinase-9 Concentration Predicts Hemorrhagic Transformation in Acute Ischemic Stroke. Stroke 34, 40-46.

Champagne, B., Tremblay, P., Cantin, A., and St. Pierre, Y. (1998). Proteolytic Cleavage of ICAM-1 by Human Neutrophil Elastase. The Journal of Immunology 161, 6398-6405.

Chen, F., Ohashi, N., Li, W., Eckman, C., and Nguyen, J.H. (2009). Disruptions of occludin and claudin5 in brain endothelial cells in vitro and in brains of mice with acute liver failure. Hepatology 50, 19141923. 
Chen, Y.H., Lu, Q., Goodenough, D.A., and Jeansonne, B. (2002). Nonreceptor tyrosine kinase c-Yes interacts with occludin during tight junction formation in canine kidney epithelial cells. Mol Biol Cell 13, 1227-1237.

Chenoweth, D.E., Goodman, M.G., and Weigle, W.O. (1982). Demonstration of a specific receptor for human C5a anaphylatoxin on murine macrophages. J Exp Med 156, 68-78.

Chenoweth, D.E., and Hugli, T.E. (1978). Demonstration of specific C5a receptor on intact human polymorphonuclear leukocytes. Proc Natl Acad Sci U S A 75, 3943-3947.

Chertov, O., Ueda, H., Xu, L.L., Tani, K., Murphy, W.J., Wang, J.M., Howard, O.M.Z., Sayers, T.J., and Oppenheim, J.J. (1997). Identification of Human Neutrophil-derived Cathepsin G and Azurocidin/CAP37 as Chemoattractants for Mononuclear Cells and Neutrophils. J Exp Med 186, 739747.

Chigaev, A., Zwartz, G., Graves, S.W., Dwyer, D.C., Tsuji, H., Foutz, T.D., Edwards, B.S., Prossnitz, E.R., Larson, R.S., and Sklar, L.A. (2003). Alpha4beta1 integrin affinity changes govern cell adhesion. J Biol Chem 278, 38174-38182.

Citi, S., Spadaro, D., Schneider, Y., Stutz, J., and Pulimeno, P. (2011). Regulation of small GTPases at epithelial cell-cell junctions. Mol Membr Biol 28, 427-444.

Claudio, L., Kress, Y., Factor, J., and Brosnan, C.F. (1990). Mechanisms of edema formation in experimental autoimmune encephalomyelitis. The contribution of inflammatory cells. Am J Pathol $137,1033-1045$.

Claudio, L., Kress, Y., Norton, W.T., and Brosnan, C.F. (1989). Increased vesicular transport and decreased mitochondrial content in blood-brain barrier endothelial cells during experimental autoimmune encephalomyelitis. Am J Pathol 135, 1157-1168.

Clements, J.M., Cossins, J.A., Wells, G.M., Corkill, D.J., Helfrich, K., Wood, L.M., Pigott, R., Stabler, G., Ward, G.A., Gearing, A.J., et al. (1997). Matrix metalloproteinase expression during experimental autoimmune encephalomyelitis and effects of a combined matrix metalloproteinase and tumour necrosis factor-alpha inhibitor. J Neuroimmunol 74, 85-94.

Cloys, D., and Netsky, M. (1970). Neuromyelitis optica, Vol 9 (Amsterdam: North Holland).

Cramer, E., Pryzwansky, K.B., Villeval, J.L., Testa, U., and Breton-Gorius, J. (1985). Ultrastructural localization of lactoferrin and myeloperoxidase in human neutrophils by immunogold. Blood 65, 423432.

Cree, B.A.C., Lamb, S., Morgan, K., Chen, A., Waubant, E., and Genain, C. (2005). An open label study of the effects of rituximab in neuromyelitis optica. Neurology $64,1270-1272$.

Cui, J., Chen, S., Zhang, C., Meng, F., Wu, W., Hu, R., Hadass, O., Lehmidi, T., Blair, G.J., Lee, M., et al. (2012). Inhibition of MMP-9 by a selective gelatinase inhibitor protects neurovasculature from embolic focal cerebral ischemia. Mol Neurodegener 7, 21.

Daneman, R., Agalliu, D., Zhou, L., Kuhnert, F., Kuo, C.J., and Barres, B.A. (2009). Wnt/beta-catenin signaling is required for CNS, but not non-CNS, angiogenesis. Proc Natl Acad Sci U S A 106, 641-646.

Daneman, R., Zhou, L., Agalliu, D., Cahoy, J.D., Kaushal, A., and Barres, B.A. (2010a). The Mouse Blood-Brain Barrier Transcriptome: A New Resource for Understanding the Development and Function of Brain Endothelial Cells. PLos One 5, e13741. 
Daneman, R., Zhou, L., Kebede, A.A., and Barres, B.A. (2010b). Pericytes are required for blood-brain barrier integrity during embryogenesis. Nature 468, 562-566.

de Seze, J., Stojkovic, T., Ferriby, D., Gauvrit, J.Y., Montagne, C., Mounier-Vehier, F., Verier, A., Pruvo, J.P., Hache, J.C., and Vermersch, P. (2002). Devic's neuromyelitis optica: clinical, laboratory, MRI and outcome profile. J Neurol Sci 197, 57-61.

de Vries, H.E., Blom-Roosemalen, M.C., van Oosten, M., de Boer, A.G., van Berkel, T.J., Breimer, D.D., and Kuiper, J. (1996). The influence of cytokines on the integrity of the blood-brain barrier in vitro. J Neuroimmunol 64, 37-43.

Demeuse, P., Kerkhofs, A., Struys-Ponsar, C., Knoops, B., Remacle, C., and van den Bosch de Aguilar, P. (2002). Compartmentalized coculture of rat brain endothelial cells and astrocytes: a syngenic model to study the blood-brain barrier. J Neurosci Methods 121, 21-31.

Detmers, P.A., Zhou, D., Powell, D., Lichenstein, H., Kelley, M., and Pironkova, R. (1995). Endotoxin receptors (CD14) are found with CD16 (Fc gamma RIII) in an intracellular compartment of neutrophils that contains alkaline phosphatase. J Immunol 155, 2085-2095.

Devic, E. (1894). Myélite subaiguë compliquée de névrite optique. Le Bulletin Médicale 8, 1033-1034.

Dewald, B., Bretz, U., and Baggiolini, M. (1982). Release of gelatinase from a novel secretory compartment of human neutrophils. J Clin Invest 70, 518-525.

Dohgu, S., Takata, F., Yamauchi, A., Nakagawa, S., Egawa, T., Naito, M., Tsuruo, T., Sawada, Y., Niwa, M., and Kataoka, Y. (2005). Brain pericytes contribute to the induction and up-regulation of bloodbrain barrier functions through transforming growth factor-beta production. Brain Res 1038, 208215.

Drin, G., Cottin, S., Blanc, E., Rees, A.R., and Temsamani, J. (2003). Studies on the internalization mechanism of cationic cell-penetrating peptides. J Biol Chem 278, 31192-31201.

Dubois, B., Masure, S., Hurtenbach, U., Paemen, L., Heremans, H., van den Oord, J., Sciot, R., Meinhardt, T., Hämmerling, G., Opdenakker, G., et al. (1999). Resistance of young gelatinase Bdeficient mice to experimental autoimmune encephalomyelitis and necrotizing tail lesions. J Clin Invest 104, 1507-1515.

Durieu-Trautmann, O., Chaverot, N., Cazaubon, S., Strosberg, A.D., and Couraud, P.O. (1994). Intercellular adhesion molecule 1 activation induces tyrosine phosphorylation of the cytoskeletonassociated protein cortactin in brain microvessel endothelial cells. J Biol Chem 269, 12536-12540.

Ehrlich, P. (1885). Das Sauerstoff-Bedürfnis des Organismus. Eine farbenanalytische Studie (Verlag von August Hirschwald, Berlin).

Eisenblätter, T., Hüwel, S., and Galla, H.-J. (2003). Characterisation of the brain multidrug resistance protein (BMDP/ABCG2/BCRP) expressed at the blood-brain barrier. Brain Research 971, 221-231.

Enge, M., Bjarnegård, M., Gerhardt, H., Gustafsson, E., Kalén, M., Asker, N., Hammes, H.P., Shani, M., Fässler, R., and Betsholtz, C. (2002). Endothelium-specific platelet-derived growth factor-B ablation mimics diabetic retinopathy, $\mathrm{Vol} 21$.

Errede, M., Girolamo, F., Ferrara, G., Strippoli, M., Morando, S., Boldrin, V., Rizzi, M., Uccelli, A., Perris, R., Bendotti, C., et al. (2012). Blood-brain barrier alterations in the cerebral cortex in experimental autoimmune encephalomyelitis. J Neuropathol Exp Neurol 71, 840-854. 
Etienne-Manneville, S., Manneville, J.B., Adamson, P., Wilbourn, B., Greenwood, J., and Couraud, P.O. (2000). ICAM-1-coupled cytoskeletal rearrangements and transendothelial lymphocyte migration involve intracellular calcium signaling in brain endothelial cell lines. J Immunol 165, 3375-3383.

Feeney, J.F., and Watterson, R.L. (1946). The development of the vascular pattern within the walls of the central nervous system of the chick embryo. Journal of Morphology 78, 231-303.

Ferrari, C.C., Depino, A.M., Prada, F., Muraro, N., Campbell, S., Podhajcer, O., Perry, V.H., Anthony, D.C., and Pitossi, F.J. (2004). Reversible Demyelination, Blood-Brain Barrier Breakdown, and Pronounced Neutrophil Recruitment Induced by Chronic IL-1 Expression in the Brain. Am J Pathol $165,1827-1837$.

Filardy, A.A., Pires, D.R., Nunes, M.P., Takiya, C.M., Freire-de-Lima, C.G., Ribeiro-Gomes, F.L., and DosReis, G.A. (2010). Proinflammatory clearance of apoptotic neutrophils induces an IL-12(low)IL10 (high) regulatory phenotype in macrophages. J Immunol 185, 2044-2050.

Finch, A.M., Wong, A.K., Paczkowski, N.J., Wadi, S.K., Craik, D.J., Fairlie, D.P., and Taylor, S.M. (1999). Low-molecular-weight peptidic and cyclic antagonists of the receptor for the complement factor C5a. J Med Chem 42, 1965-1974.

Foreman, K.E., Vaporciyan, A.A., Bonish, B.K., Jones, M.L., Johnson, K.J., Glovsky, M.M., Eddy, S.M., and Ward, P.A. (1994). C5a-induced expression of P-selectin in endothelial cells. J Clin Invest 94, 1147-1155.

Fuortes, M., Jin, W.W., and Nathan, C. (1993). Adhesion-dependent protein tyrosine phosphorylation in neutrophils treated with tumor necrosis factor. J Cell Biol 120, 777-784.

Furuse, M., Fujimoto, K., Sato, N., Hirase, T., and Tsukita, S. (1996). Overexpression of occludin, a tight junction-associated integral membrane protein, induces the formation of intracellular multilamellar bodies bearing tight junction-like structures. J Cell Sci 109 ( Pt 2), 429-435.

Furuse, M., Fujita, K., Hiiragi, T., Fujimoto, K., and Tsukita, S. (1998a). Claudin-1 and -2: novel integral membrane proteins localizing at tight junctions with no sequence similarity to occludin. J Cell Biol $141,1539-1550$.

Furuse, M., Hirase, T., Itoh, M., Nagafuchi, A., Yonemura, S., and Tsukita, S. (1993). Occludin: a novel integral membrane protein localizing at tight junctions. J Cell Biol 123, 1777-1788.

Furuse, M., Sasaki, H., Fujimoto, K., and Tsukita, S. (1998b). A Single Gene Product, Claudin-1 or -2, Reconstitutes Tight Junction Strands and Recruits Occludin in Fibroblasts. J Cell Biol 143, 391-401.

Garcia, J.H., Liu, K.F., Yoshida, Y., Lian, J., Chen, S., and del Zoppo, G.J. (1994). Influx of leukocytes and platelets in an evolving brain infarct (Wistar rat). Am J Pathol 144, 188-199.

Gasque, P., Chan, P., Fontaine, M., Ischenko, A., Lamacz, M., Gotze, O., and Morgan, B.P. (1995). Identification and characterization of the complement $\mathrm{C} 5$ a anaphylatoxin receptor on human astrocytes. J Immunol 155, 4882-4889.

Gault, F. (1894). De la neuromyélite optique aiguë. Thése: Faculté de Médecine et de Pharmacie.

Gautam, N., Hedqvist, P., and Lindbom, L. (1998). Kinetics of leukocyte-induced changes in endothelial barrier function. Br J Pharmacol 125, 1109-1114.

Gautam, N., Herwald, H., Hedqvist, P., and Lindbom, L. (2000). Signaling via beta(2) integrins triggers neutrophil-dependent alteration in endothelial barrier function. J Exp Med 191, 1829-1839. 
Gijbels, K., Galardy, R.E., and Steinman, L. (1994). Reversal of experimental autoimmune encephalomyelitis with a hydroxamate inhibitor of matrix metalloproteases. The Journal of Clinical Investigation 94, 2177-2182.

Gijbels, K., Masure, S., Carton, H., and Opdenakker, G. (1992). Gelatinase in the cerebrospinal fluid of patients with multiple sclerosis and other inflammatory neurological disorders. J Neuroimmunol 41, 29-34.

Goldmann, E. (1913). Vitalfärbungen am Zentralnervensystem. Beitrag zur Physio-Pathologie des Plexus Choroideus and der Hirnhäute. Abhandlungen der königlich preußischen Akademie der Wissenschaften. Physikalisch-Mathematische Classe 1, 1-64.

Goldstein, I.M., Roos, D., Kaplan, H.B., and Weissmann, G. (1975). Complement and immunoglobulins stimulate superoxide production by human leukocytes independently of phagocytosis. J Clin Invest 56, 1155-1163.

Gopalakrishnan, S., Raman, N., Atkinson, S.J., and Marrs, J.A. (1998). Rho GTPase signaling regulates tight junction assembly and protects tight junctions during ATP depletion. Am J Physiol 275, C798809.

Gorboulev, V., Ulzheimer, J.C., Akhoundova, A., Ulzheimer-Teuber, I., Karbach, U., Quester, S., Baumann, C., Lang, F., Busch, A.E., and Koepsell, H. (1997). Cloning and characterization of two human polyspecific organic cation transporters. DNA Cell Biol 16, 871-881.

Gorina, R., Lyck, R., Vestweber, D., and Engelhardt, B. (2014). 32 Integrin-Mediated Crawling on Endothelial ICAM-1 and ICAM-2 Is a Prerequisite for Transcellular Neutrophil Diapedesis across the Inflamed Blood-Brain Barrier. The Journal of Immunology 192, 324-337.

Greenwood, J., Amos, C.L., Walters, C.E., Couraud, P.O., Lyck, R., Engelhardt, B., and Adamson, P. (2003). Intracellular domain of brain endothelial intercellular adhesion molecule-1 is essential for $T$ lymphocyte-mediated signaling and migration. J Immunol 171, 2099-2108.

Greenwood, J., Howes, R., and Lightman, S. (1994). The blood-retinal barrier in experimental autoimmune uveoretinitis. Leukocyte interactions and functional damage. Lab Invest 70, 39-52.

Griffiths, M., Beaumont, N., Yao, S.Y., Sundaram, M., Boumah, C.E., Davies, A., Kwong, F.Y., Coe, I., Cass, C.E., Young, J.D., et al. (1997). Cloning of a human nucleoside transporter implicated in the cellular uptake of adenosine and chemotherapeutic drugs. Nat Med 3, 89-93.

Grossman, R.I., Gonzalez-Scarano, F., Atlas, S.W., Galetta, S., and Silberberg, D.H. (1986). Multiple sclerosis: gadolinium enhancement in MR imaging. Radiology 161, 721-725.

Gu, Z., Cui, J., Brown, S., Fridman, R., Mobashery, S., Strongin, A.Y., and Lipton, S.A. (2005). A Highly Specific Inhibitor of Matrix Metalloproteinase-9 Rescues Laminin from Proteolysis and Neurons from Apoptosis in Transient Focal Cerebral Ischemia. The Journal of Neuroscience 25, 6401-6408.

Gumbiner, B., Lowenkopf, T., and Apatira, D. (1991). Identification of a 160-kDa polypeptide that binds to the tight junction protein ZO-1. Proceedings of the National Academy of Sciences 88, 34603464.

Hadass, O., Tomlinson, B.N., Gooyit, M., Chen, S., Purdy, J.J., Walker, J.M., Zhang, C., Giritharan, A.B., Purnell, W., Robinson, C.R., II, et al. (2013). Selective Inhibition of Matrix Metalloproteinase-9 Attenuates Secondary Damage Resulting from Severe Traumatic Brain Injury. PLos One 8, e76904. 
Hafezi-Moghadam, A., Thomas, K.L., and Wagner, D.D. (2007). ApoE deficiency leads to a progressive age-dependent blood-brain barrier leakage. Am J Physiol Cell Physiol 292, C1256-1262.

Harhaj, N.S., Felinski, E.A., Wolpert, E.B., Sundstrom, J.M., Gardner, T.W., and Antonetti, D.A. (2006). VEGF activation of protein kinase $C$ stimulates occludin phosphorylation and contributes to endothelial permeability. Invest Ophthalmol Vis Sci 47, 5106-5115.

Hellström, M., Gerhardt, H., Kalén, M., Li, X., Eriksson, U., Wolburg, H., and Betsholtz, C. (2001). Lack of Pericytes Leads to Endothelial Hyperplasia and Abnormal Vascular Morphogenesis. The Journal of Cell Biology 153, 543-554.

Hellström, M., Kalen, M., Lindahl, P., Abramsson, A., and Betsholtz, C. (1999). Role of PDGF-B and PDGFR-beta in recruitment of vascular smooth muscle cells and pericytes during embryonic blood vessel formation in the mouse. Development 126, 3047-3055.

Hermant, B., Bibert, S., Concord, E., Dublet, B., Weidenhaupt, M., Vernet, T., and Gulino-Debrac, D. (2003). Identification of proteases involved in the proteolysis of vascular endothelium cadherin during neutrophil transmigration. J Biol Chem 278, 14002-14012.

Hewson, A.K., Smith, T., Leonard, J.P., and Cuzner, M.L. (1995). Suppression of experimental allergic encephalomyelitis in the Lewis rat by the matrix metalloproteinase inhibitor Ro31-9790. Inflamm Res 44, 345-349.

Hinson, S., Pittock, S., Lucchinetti, C., Roemer, S., Fryer, J., Kryzer, T., and Lennon, V. (2007). Pathogenic potential of IgG binding to water channel extracellular domain in neuromyelitis optica. Neurology 69, 2221 - 2231.

Holash, J.A., Noden, D.M., and Stewart, P.A. (1993). Re-evaluating the role of astrocytes in bloodbrain barrier induction. Dev Dyn 197, 14-25.

Horstmann, S., Kalb, P., Koziol, J., Gardner, H., and Wagner, S. (2003). Profiles of Matrix Metalloproteinases, Their Inhibitors, and Laminin in Stroke Patients: Influence of Different Therapies. Stroke 34, 2165-2170.

Hosokawa, T., Nakajima, H., Doi, Y., Sugino, M., Kimura, F., Hanafusa, T., and Takahashi, T. (2011). Increased serum matrix metalloproteinase-9 in neuromyelitis optica: Implication of disruption of blood-brain barrier. Journal of Neuroimmunology 236, 81-86.

Ikegame, Y., Yamashita, K., Hayashi, S., Yoshimura, S., Nakashima, S., and Iwama, T. (2010). Neutrophil elastase inhibitor prevents ischemic brain damage via reduction of vasogenic edema. Hypertens Res 33, 703-707.

Ikenouchi, J., Furuse, M., Furuse, K., Sasaki, H., and Tsukita, S. (2005). Tricellulin constitutes a novel barrier at tricellular contacts of epithelial cells. J Cell Biol 171, 939-945.

Ito, S., Mori, M., Makino, T., Hayakawa, S., and Kuwabara, S. (2009). "Cloud-like enhancement" is a magnetic resonance imaging abnormality specific to neuromyelitis optica. Ann Neurol 66, 425-428.

Janzer, R.C., and Raff, M.C. (1987). Astrocytes induce blood-brain barrier properties in endothelial cells. Nature $325,253-257$.

Jarius, S., Franciotta, D., Paul, F., Bergamaschi, R., Rommer, P.S., Ruprecht, K., Ringelstein, M., Aktas, O., Kristoferitsch, W., and Wildemann, B. (2012a). Testing for antibodies to human aquaporin-4 by ELISA: sensitivity, specificity, and direct comparison with immunohistochemistry. J Neurol Sci 320, 3237. 
Jarius, S., Paul, F., Franciotta, D., Ruprecht, K., Ringelstein, M., Bergamaschi, R., Rommer, P., Kleiter, I., Stich, O., Reuss, R., et al. (2011). Cerebrospinal fluid findings in aquaporin-4 antibody positive neuromyelitis optica: Results from 211 lumbar punctures. Journal of the Neurological Sciences 306, 82-90.

Jarius, S., Ruprecht, K., Wildemann, B., Kuempfel, T., Ringelstein, M., Geis, C., Kleiter, I., Kleinschnitz, C., Berthele, A., Brettschneider, J., et al. (2012b). Contrasting disease patterns in seropositive and seronegative neuromyelitis optica: a multicentre study of 175 patients. J Neuroinflammation 9, 14.

Jiang, X., Namura, S., and Nagata, I. (2001). Matrix metalloproteinase inhibitor KB-R7785 attenuates brain damage resulting from permanent focal cerebral ischemia in mice. Neurosci Lett 305, 41-44.

Joice, S.L., Mydeen, F., Couraud, P.-O., Weksler, B.B., Romero, I.A., Fraser, P.A., and Easton, A.S. (2009). Modulation of blood-brain barrier permeability by neutrophils: in vitro and in vivo studies. Brain Research 1298, 13-23.

Juliano, R.L., and Ling, V. (1976). A surface glycoprotein modulating drug permeability in Chinese hamster ovary cell mutants. Biochim Biophys Acta 455, 152-162.

Kago, T., Takagi, N., Date, I., Takenaga, Y., Takagi, K., and Takeo, S. (2006). Cerebral ischemia enhances tyrosine phosphorylation of occludin in brain capillaries. Biochem Biophys Res Commun 339, 1197-1203.

Kallmann, B.A., Wagner, S., Hummel, V., Buttmann, M., Bayas, A., Tonn, J.C., and Rieckmann, P. (2002). Characteristic gene expression profile of primary human cerebral endothelial cells. FASEB J 16, 589-591.

Kandagaddala, L.D., Kang, M.-J., Chung, B.C., Patterson, T.A., and Kwon, O.-S. (2012). Expression and activation of matrix metalloproteinase- 9 and NADPH oxidase in tissues and plasma of experimental autoimmune encephalomyelitis in mice. Experimental and Toxicologic Pathology 64, 109-114.

Kawabata, K., Suzuki, M., Sugitani, M., Imaki, K., Toda, M., and Miyamoto, T. (1991). ONO-5046, a novel inhibitor of human neutrophil elastase. Biochem Biophys Res Commun 177, 814-820.

Kaynar, A.M., Houghton, A.M., Lum, E.H., Pitt, B.R., and Shapiro, S.D. (2008). Neutrophil elastase is needed for neutrophil emigration into lungs in ventilator-induced lung injury. Am J Respir Cell Mol Biol 39, 53-60.

Kernen, P., Wymann, M.P., von Tscharner, V., Deranleau, D.A., Tai, P.C., Spry, C.J., Dahinden, C.A., and Baggiolini, M. (1991). Shape changes, exocytosis, and cytosolic free calcium changes in stimulated human eosinophils. J Clin Invest 87, 2012-2017.

Kieseier, B.C., Kiefer, R., Clements, J.M., Miller, K., Wells, G.M., Schweitzer, T., Gearing, A.J., and Hartung, H.P. (1998). Matrix metalloproteinase- 9 and -7 are regulated in experimental autoimmune encephalomyelitis, Vol 121.

Kim, H.J., Paul, F., Lana-Peixoto, M.A., Tenembaum, S., Asgari, N., Palace, J., Klawiter, E.C., Sato, D.K., de Seze, J., Wuerfel, J., et al. (2015). MRI characteristics of neuromyelitis optica spectrum disorder: An international update. Neurology 84, 1165-1173.

Kim, W., Lee, J.E., Li, X.F., Kim, S.H., Han, B.G., Lee, B.I., Kim, J.K., Choi, K., and Kim, H.J. (2012). Quantitative measurement of anti-aquaporin-4 antibodies by enzyme-linked immunosorbent assay using purified recombinant human aquaporin-4. Mult Scler 18, 578-586. 
King, J.S., and Schwyn, R.C. (1970). The fine structure of neuroglial cells and pericytes in the primate red nucleus and substantia nigra. Z Zellforsch Mikrosk Anat 106, 309-321.

Kinoshita, M., Nakatsuji, Y., Kimura, T., Moriya, M., Takata, K., Okuno, T., Kumanogoh, A., Kajiyama, K., Yoshikawa, H., and Sakoda, S. (2009). Neuromyelitis optica: Passive transfer to rats by human immunoglobulin. Biochem Biophys Res Commun 386, 623-627.

Kinoshita, M., Nakatsuji, Y., Kimura, T., Moriya, M., Takata, K., Okuno, T., Kumanogoh, A., Kajiyama, K., Yoshikawa, H., and Sakoda, S. (2010). Anti-aquaporin-4 antibody induces astrocytic cytotoxicity in the absence of CNS antigen-specific T cells. Biochemical and Biophysical Research Communications 394, 205-210.

Kirk, J., Plumb, J., Mirakhur, M., and McQuaid, S. (2003). Tight junctional abnormality in multiple sclerosis white matter affects all calibres of vessel and is associated with blood-brain barrier leakage and active demyelination. The Journal of Pathology 201, 319-327.

Kjeldsen, L., Bainton, D.F., Sengelov, H., and Borregaard, N. (1993). Structural and functional heterogeneity among peroxidase-negative granules in human neutrophils: identification of a distinct gelatinase-containing granule subset by combined immunocytochemistry and subcellular fractionation. Blood 82, 3183-3191.

Koistinaho, M., Malm, T.M., Kettunen, M.I., Goldsteins, G., Starckx, S., Kauppinen, R.A., Opdenakker, G., and Koistinaho, J. (2005). Minocycline protects against permanent cerebral ischemia in wild type but not in matrix metalloprotease-9-deficient mice. J Cereb Blood Flow Metab 25, 460-467.

Kooij, G., Kopplin, K., Blasig, R., Stuiver, M., Koning, N., Goverse, G., van der Pol, S.M.A., van het Hof, B., Gollasch, M., Drexhage, J.A.R., et al. (2013). Disturbed function of the blood-cerebrospinal fluid barrier aggravates neuro-inflammation. Acta Neuropathologica 128, 267-277.

Kratzer, I., Vasiljevic, A., Rey, C., Fevre-Montange, M., Saunders, N., Strazielle, N., and Ghersi-Egea, J.F. (2012). Complexity and developmental changes in the expression pattern of claudins at the bloodCSF barrier. Histochemistry and Cell Biology 138, 861-879.

Krum, J.M. (1994). Experimental gliopathy in the adult rat CNS: effect on the blood-spinal cord barrier. Glia 11, 354-366.

Krum, J.M. (1996). Effect of astroglial degeneration on neonatal blood-brain barrier marker expression. Exp Neurol 142, 29-35.

Krum, J.M., and Rosenstein, J.M. (1989). The fine structure of vascular-astroglial relations in transplanted fetal neocortex. Exp Neurol 103, 203-212.

Krum, J.M., and Rosenstein, J.M. (1993). Effect of astroglial degeneration on the blood-brain barrier to protein in neonatal rats. Brain Res Dev Brain Res 74, 41-50.

Kurihara, T., Warr, G., Loy, J., and Bravo, R. (1997). Defects in Macrophage Recruitment and Host Defense in Mice Lacking the CCR2 Chemokine Receptor. The Journal of Experimental Medicine 186, 1757-1762.

Kuroiwa, Y., Igata, A., Itahara, K., Koshijima, S., and Tsubaki, T. (1975). Nationwide survey of multiple sclerosis in Japan. Clinical analysis of 1,084 cases. Neurology 25, 845-851.

Kuziel, W.A., Morgan, S.J., Dawson, T.C., Griffin, S., Smithies, O., Ley, K., and Maeda, N. (1997). Severe reduction in leukocyte adhesion and monocyte extravasation in mice deficient in CC chemokine receptor 2. Proc Natl Acad Sci U S A 94, 12053-12058. 
Lacy, M., Jones, J., Whittemore, S.R., Haviland, D.L., Wetsel, R.A., and Barnum, S.R. (1995). Expression of the receptors for the $\mathrm{C} 5 \mathrm{a}$ anaphylatoxin, interleukin-8 and FMLP by human astrocytes and microglia. J Neuroimmunol 61, 71-78.

Laudes, I.J., Chu, J.C., Huber-Lang, M., Guo, R.F., Riedemann, N.C., Sarma, J.V., Mahdi, F., Murphy, H.S., Speyer, C., Lu, K.T., et al. (2002). Expression and function of C5a receptor in mouse microvascular endothelial cells. J Immunol 169, 5962-5970.

Lee, S.-W., Kim, W.J., Choi, Y.K., Song, H.S., Son, M.J., Gelman, I.H., Kim, Y.-J., and Kim, K.-W. (2003). SSeCKS regulates angiogenesis and tight junction formation in blood-brain barrier. Nat Med 9, 900906.

Lennon, V., Kryzer, T., Pittock, S., Verkman, A., and Hinson, S. (2005). IgG marker of optic-spinal multiple sclerosis binds to the aquaporin-4 water channel. J Exp Med 202, 473 - 477.

Lennon, V., Wingerchuk, D., Kryzer, T., Pittock, S., Lucchinetti, C., Fujihara, K., Nakashima, I., and Weinshenker, B. (2004). A serum autoantibody marker of neuromyelitis optica: distinction from multiple sclerosis. The Lancet 364, 2106-2112.

Lennon, V.A. (2005). IgG marker of optic-spinal multiple sclerosis binds to the aquaporin-4 water channel. Journal of Experimental Medicine 202, 473-477.

Levy, M., and Mealy, M.A. (2014). Purified human C1-esterase inhibitor is safe in acute relapses of neuromyelitis optica. Neurol Neuroimmunol Neuroinflamm 1.

Lewandowski, M. (1900). Zur Lehre von der Cerebrospinalflüssigkeit. Z Klein Forsch 40, 480-494.

Liebner, S., Corada, M., Bangsow, T., Babbage, J., Taddei, A., Czupalla, C.J., Reis, M., Felici, A., Wolburg, H., Fruttiger, M., et al. (2008). Wnt/beta-catenin signaling controls development of the blood-brain barrier. J Cell Biol 183, 409-417.

Liebner, S., Fischmann, A., Rascher, G., Duffner, F., Grote, E.H., Kalbacher, H., and Wolburg, H. (2000a). Claudin-1 and claudin-5 expression and tight junction morphology are altered in blood vessels of human glioblastoma multiforme. Acta Neuropathologica 100, 323-331.

Liebner, S., Kniesel, U., Kalbacher, H., and Wolburg, H. (2000b). Correlation of tight junction morphology with the expression of tight junction proteins in blood-brain barrier endothelial cells. Eur J Cell Biol 79, 707-717.

Lindahl, P., Johansson, B.R., Leveen, P., and Betsholtz, C. (1997). Pericyte loss and microaneurysm formation in PDGF-B-deficient mice. Science 277, 242-245.

Lindberg, R.L.P., De Groot, C.J.A., Montagne, L., Freitag, P., van der Valk, P., Kappos, L., and Leppert, D. (2001). The expression profile of matrix metalloproteinases (MMPs) and their inhibitors (TIMPs) in lesions and normal appearing white matter of multiple sclerosis, Vol 124.

Lischper, M., Beuck, S., Thanabalasundaram, G., Pieper, C., and Galla, H.-J. (2010). Metalloproteinase mediated occludin cleavage in the cerebral microcapillary endothelium under pathological conditions. Brain Research 1326, 114-127.

Liu, W., Hendren, J., Qin, X.J., Shen, J., and Liu, K.J. (2009). Normobaric hyperoxia attenuates early blood-brain barrier disruption by inhibiting MMP-9-mediated occludin degradation in focal cerebral ischemia. J Neurochem 108, 811-820. 
Liuzzi, G.M., Mastroianni, C.M., Santacroce, M.P., Fanelli, M., D'Agostino, C., Vullo, V., and Riccio, P. (2000). Increased activity of matrix metalloproteinases in the cerebrospinal fluid of patients with HIVassociated neurological diseases. J Neurovirol 6, 156-163.

Lloyds, D., Brindle, N.P., and Hallett, M.B. (1995). Priming of human neutrophils by tumour necrosis factor-alpha and substance $P$ is associated with tyrosine phosphorylation. Immunology 84, 220-226.

Lombardi, A., Cantini, G., Mello, T., Francalanci, M., Gelmini, S., Cosmi, L., Santarlasci, V., Degl'Innocenti, S., Luciani, P., Deledda, C., et al. (2009). Molecular mechanisms underlying the proinflammatory synergistic effect of tumor necrosis factor alpha and interferon gamma in human microvascular endothelium. Eur J Cell Biol 88, 731-742.

Lorenzl, S., Albers, D.S., Narr, S., Chirichigno, J., and Beal, M.F. (2002). Expression of MMP-2, MMP-9, and MMP-1 and Their Endogenous Counterregulators TIMP-1 and TIMP-2 in Postmortem Brain Tissue of Parkinson's Disease. Experimental Neurology 178, 13-20.

Lossinsky, A.S., Badmajew, V., Robson, J.A., Moretz, R.C., and Wisniewski, H.M. (1989). Sites of egress of inflammatory cells and horseradish peroxidase transport across the blood-brain barrier in a murine model of chronic relapsing experimental allergic encephalomyelitis. Acta Neuropathol 78, 359-371.

Lucchinetti, C., Bruck, W., Parisi, J., Scheithauer, B., Rodriguez, M., and Lassmann, H. (2000). Heterogeneity of multiple sclerosis lesions: implications for the pathogenesis of demyelination. Ann Neurol 47, 707-717.

Lucchinetti, C.F., Mandler, R.N., McGavern, D., Bruck, W., Gleich, G., Ransohoff, R.M., Trebst, C., Weinshenker, B., Wingerchuk, D., Parisi, J.E., et al. (2002). A role for humoral mechanisms in the pathogenesis of Devic's neuromyelitis optica. Brain 125, 1450-1461.

Luissint, A.C., Federici, C., Guillonneau, F., Chrétien, F., Camoin, L., Glacial, F., Ganeshamoorthy, K., and Couraud, P.O. (2012). Guanine nucleotide-binding protein Gai2: a new partner of claudin-5 that regulates tight junction integrity in human brain endothelial cells. J Cereb Blood Flow Metab 32, 860873.

Lyck, R., Reiss, Y., Gerwin, N., Greenwood, J., Adamson, P., and Engelhardt, B. (2003). T-cell interaction with ICAM-1/ICAM-2 double-deficient brain endothelium in vitro: the cytoplasmic tail of endothelial ICAM-1 is necessary for transendothelial migration of T cells. Blood 102, 3675-3683.

Lyck, R., Ruderisch, N., Moll, A.G., Steiner, O., Cohen, C.D., Engelhardt, B., Makrides, V., and Verrey, F. (2009). Culture-induced changes in blood-brain barrier transcriptome: implications for amino-acid transporters in vivo. J Cereb Blood Flow Metab 29, 1491-1502.

Malemud, C.J., and Janoff, A. (1975). Human polymorphonuclear leukocyte elastase and cathepsin G mediate the degradation of lapine articular cartilage proteoglycan. Ann N Y Acad Sci 256, 254-262.

Mandler, R.N., Davis, L.E., Jeffery, D.R., and Kornfeld, M. (1993). Devic's neuromyelitis optica: a clinicopathological study of 8 patients. Ann Neurol 34, 162-168.

Mandler, R.N., Dencoff, J.D., Midani, F., Ford, C.C., Ahmed, W., and Rosenberg, G.A. (2001). Matrix metalloproteinases and tissue inhibitors of metalloproteinases in cerebrospinal fluid differ in multiple sclerosis and Devic's neuromyelitis optica, Vol 124.

Matsuo, Y., Onodera, H., Shiga, Y., Nakamura, M., Ninomiya, M., Kihara, T., and Kogure, K. (1994). Correlation between myeloperoxidase-quantified neutrophil accumulation and ischemic brain injury in the rat. Effects of neutrophil depletion. Stroke 25, 1469-1475. 
Maxwell, K., Berliner, J.A., and Cancilla, P.A. (1987). Induction of gamma-glutamyl transpeptidase in cultured cerebral endothelial cells by a product released by astrocytes. Brain Res 410, 309-314.

McCarthy, K.M., Skare, I.B., Stankewich, M.C., Furuse, M., Tsukita, S., Rogers, R.A., Lynch, R.D., and Schneeberger, E.E. (1996). Occludin is a functional component of the tight junction. Journal of Cell Science 109, 2287-2298.

McKimmie, C.S., Fraser, A.R., Hansell, C., Gutierrez, L., Philipsen, S., Connell, L., Rot, A., KurowskaStolarska, M., Carreno, P., Pruenster, M., et al. (2008). Hemopoietic cell expression of the chemokine decoy receptor D6 is dynamic and regulated by GATA1. J Immunol 181, 3353-3363.

McMenamin, P.G., Forrester, J.V., Steptoe, R.J., and Dua, H.S. (1992). Ultrastructural pathology of experimental autoimmune uveitis. Quantitative evidence of activation and possible high endothelial venule-like changes in retinal vascular endothelium. Lab Invest 67, 42-55.

Mealy, M.A., Wingerchuk, D.M., Greenberg, B.M., and Levy, M. (2012). Epidemiology of neuromyelitis optica in the united states: A multicenter analysis. Archives of Neurology 69, 11761180.

Methia, N., Andre, P., Hafezi-Moghadam, A., Economopoulos, M., Thomas, K.L., and Wagner, D.D. (2001). ApoE deficiency compromises the blood brain barrier especially after injury. Mol Med 7, 810815.

Miller, L.J., Bainton, D.F., Borregaard, N., and Springer, T.A. (1987). Stimulated mobilization of monocyte Mac-1 and p150,95 adhesion proteins from an intracellular vesicular compartment to the cell surface. J Clin Invest 80, 535-544.

Mineta, K., Yamamoto, Y., Yamazaki, Y., Tanaka, H., Tada, Y., Saito, K., Tamura, A., Igarashi, M., Endo, T., Takeuchi, K., et al. (2011). Predicted expansion of the claudin multigene family. FEBS Lett 585, 606-612.

Misu, T., Fujihara, K., Kakita, A., Konno, H., Nakamura, M., Watanabe, S., Takahashi, T., Nakashima, I., Takahashi, H., and Itoyama, Y. (2007). Loss of aquaporin 4 in lesions of neuromyelitis optica: distinction from multiple sclerosis. Brain 130, 1224-1234.

Misu, T., Fujihara, K., Nakashima, I., Sato, S., and Itoyama, Y. (2005). Intractable hiccup and nausea with periaqueductal lesions in neuromyelitis optica. Neurology 65, 1479-1482.

Mizee, M.R., Nijland, P.G., van der Pol, S.M., Drexhage, J.A., van Het Hof, B., Mebius, R., van der Valk, P., van Horssen, J., Reijerkerk, A., and de Vries, H.E. (2014). Astrocyte-derived retinoic acid: a novel regulator of blood-brain barrier function in multiple sclerosis. Acta Neuropathol 128, 691-703.

Mollnes, T.E., Brekke, O.L., Fung, M., Fure, H., Christiansen, D., Bergseth, G., Videm, V., Lappegard, K.T., Kohl, J., and Lambris, J.D. (2002). Essential role of the C5a receptor in E coli-induced oxidative burst and phagocytosis revealed by a novel lepirudin-based human whole blood model of inflammation. Blood 100, 1869-1877.

Montaner, J., Rovira, A., Molina, C.A., Arenillas, J.F., Ribo, M., Chacon, P., Monasterio, J., and AlvarezSabin, J. (2003). Plasmatic Level of Neuroinflammatory Markers Predict the Extent of DiffusionWeighted Image Lesions in Hyperacute Stroke. J Cereb Blood Flow Metab 23, 1403-1407.

Morita, K., Furuse, M., Fujimoto, K., and Tsukita, S. (1999). Claudin multigene family encoding fourtransmembrane domain protein components of tight junction strands. Proc Natl Acad Sci U S A 96, 511-516. 
Morrissey, S.P., Stodal, H., Zettl, U., Simonis, C., Jung, S., Kiefer, R., Lassmann, H., Hartung, H.P., Haase, A., and Toyka, K.V. (1996). In vivo MRI and its histological correlates in acute adoptive transfer experimental allergic encephalomyelitis. Quantification of inflammation and oedema. Brain 119 ( Pt 1), 239-248.

Movat, H.Z., and Fernando, N.V. (1964). THE FINE STRUCTURE OF THE TERMINAL VASCULAR BED. IV. THE VENULES AND THEIR PERIVASCULAR CELLS (PERICYTES, ADVENTITIAL CELLS). Exp Mol Pathol 34, 98-114.

Mueckler, M., Caruso, C., Baldwin, S.A., Panico, M., Blench, I., Morris, H.R., Allard, W.J., Lienhard, G.E., and Lodish, H.F. (1985). Sequence and structure of a human glucose transporter. Science 229, 941-945.

Muradashvili, N., Benton, R.L., Saatman, K.E., Tyagi, S.C., and Lominadze, D. (2015). Ablation of matrix metalloproteinase- 9 gene decreases cerebrovascular permeability and fibrinogen deposition post traumatic brain injury in mice. Metab Brain Dis 30, 411-426.

Murakami, M., Sugita, A., Shimada, T., and Nakamura, K. (1979). Surface view of pericytes on the retinal capillary in rabbits revealed by scanning electron microscopy. Arch Histol Jpn 42, 297-303.

Murakami, T., Felinski, E.A., and Antonetti, D.A. (2009). Occludin Phosphorylation and Ubiquitination Regulate Tight Junction Trafficking and Vascular Endothelial Growth Factor-induced Permeability. Journal of Biological Chemistry 284, 21036-21046.

Murphy, G., Bretz, U., Baggiolini, M., and Reynolds, J.J. (1980). The latent collagenase and gelatinase of human polymorphonuclear neutrophil leucocytes. Biochem J 192, 517-525.

Nakagawa, S., Deli, M.A., Kawaguchi, H., Shimizudani, T., Shimono, T., Kittel, A., Tanaka, K., and Niwa, M. (2009). A new blood-brain barrier model using primary rat brain endothelial cells, pericytes and astrocytes. Neurochem Int 54, 253-263.

Nakagawa, S., Deli, M.A., Nakao, S., Honda, M., Hayashi, K., Nakaoke, R., Kataoka, Y., and Niwa, M. (2007). Pericytes from brain microvessels strengthen the barrier integrity in primary cultures of rat brain endothelial cells. Cell Mol Neurobiol 27, 687-694.

Nasdala, I., Wolburg-Buchholz, K., Wolburg, H., Kuhn, A., Ebnet, K., Brachtendorf, G., Samulowitz, U., Kuster, B., Engelhardt, B., Vestweber, D., et al. (2002). A transmembrane tight junction protein selectively expressed on endothelial cells and platelets. J Biol Chem 277, 16294-16303.

Nataf, S., Levison, S.W., and Barnum, S.R. (2001). Expression of the anaphylatoxin C5a receptor in the oligodendrocyte lineage. Brain Res 894, 321-326.

Nathan, C., Srimal, S., Farber, C., Sanchez, E., Kabbash, L., Asch, A., Gailit, J., and Wright, S.D. (1989). Cytokine-induced respiratory burst of human neutrophils: dependence on extracellular matrix proteins and CD11/CD18 integrins. J Cell Biol 109, 1341-1349.

Neumann, J., Riek-Burchardt, M., Herz, J., Doeppner, T.R., Konig, R., Hutten, H., Etemire, E., Mann, L., Klingberg, A., Fischer, T., et al. (2015). Very-late-antigen-4 (VLA-4)-mediated brain invasion by neutrophils leads to interactions with microglia, increased ischemic injury and impaired behavior in experimental stroke. Acta Neuropathol 129, 259-277.

Niimi, N., Kohyama, K., and Matsumoto, Y. (2013). Minocycline suppresses experimental autoimmune encephalomyelitis by increasing tissue inhibitors of metalloproteinases. Neuropathology 33, 612-620. 
Nishitsuji, K., Hosono, T., Nakamura, T., Bu, G., and Michikawa, M. (2011). Apolipoprotein E regulates the integrity of tight junctions in an isoform-dependent manner in an in vitro blood-brain barrier model. J Biol Chem 286, 17536-17542.

Nishiyama, S., Ito, T., Misu, T., Takahashi, T., Kikuchi, A., Suzuki, N., Jin, K., Aoki, M., Fujihara, K., and Itoyama, Y. (2009). A case of NMO seropositive for aquaporin-4 antibody more than 10 years before onset. Neurology 72, 1960-1961.

Nitta, T. (2003). Size-selective loosening of the blood-brain barrier in claudin-5-deficient mice. The Journal of Cell Biology 161, 653-660.

Nygårdas, P.T., and Hinkkanen, A.E. (2002). Up-regulation of MMP-8 and MMP-9 activity in the $\mathrm{BALB} / \mathrm{c}$ mouse spinal cord correlates with the severity of experimental autoimmune encephalomyelitis. Clin Exp Immunol 128, 245-254.

O'Riordan, J.I., Gallagher, H.L., Thompson, A.J., Howard, R.S., Kingsley, D.P., Thompson, E.J., McDonald, W.I., and Miller, D.H. (1996). Clinical, CSF, and MRI findings in Devic's neuromyelitis optica. Journal of Neurology, Neurosurgery \& Psychiatry 60, 382-387.

Ohlsson, K., and Odsson, I. (1974). The Neutral Proteases of Human Granulocytes. European Journal of Biochemistry 42, 519-527.

Ohtsuki, S., Sato, S., Yamaguchi, H., Kamoi, M., Asashima, T., and Terasaki, T. (2007). Exogenous expression of claudin-5 induces barrier properties in cultured rat brain capillary endothelial cells. Journal of Cellular Physiology 210, 81-86.

Ohtsuki, S., Yamaguchi, H., Katsukura, Y., Asashima, T., and Terasaki, T. (2008). mRNA expression levels of tight junction protein genes in mouse brain capillary endothelial cells highly purified by magnetic cell sorting. J Neurochem 104, 147-154.

Ozaki, H., Ishii, K., Horiuchi, H., Arai, H., Kawamoto, T., Okawa, K., Iwamatsu, A., and Kita, T. (1999). Cutting edge: combined treatment of TNF-alpha and IFN-gamma causes redistribution of junctional adhesion molecule in human endothelial cells. J Immunol 163, 553-557.

Pan, W., Kastin, A.J., and Brennan, J.M. (2000). Saturable entry of leukemia inhibitory factor from blood to the central nervous system. J Neuroimmunol 106, 172-180.

Pardridge, W.M., Triguero, D., Buciak, J., and Yang, J. (1990). Evaluation of cationized rat albumin as a potential blood-brain barrier drug transport vector. J Pharmacol Exp Ther 255, 893-899.

Parratt, J.D., and Prineas, J.W. (2010). Neuromyelitis optica: a demyelinating disease characterized by acute destruction and regeneration of perivascular astrocytes. Multiple Sclerosis 16, 1156-1172.

Perianayagam, M.C., Balakrishnan, V.S., Pereira, B.J., and Jaber, B.L. (2004). C5a delays apoptosis of human neutrophils via an extracellular signal-regulated kinase and Bad-mediated signalling pathway. Eur J Clin Invest 34, 50-56.

Perrière, N., Demeuse, P.H., Garcia, E., Regina, A., Debray, M., Andreux, J.P., Couvreur, P., Scherrmann, J.M., Temsamani, J., Couraud, P.O., et al. (2005). Puromycin-based purification of rat brain capillary endothelial cell cultures. Effect on the expression of blood-brain barrier-specific properties. Journal of Neurochemistry 93, 279-289.

Perriere, N., Yousif, S., Cazaubon, S., Chaverot, N., Bourasset, F., Cisternino, S., Decleves, X., Hori, S., Terasaki, T., Deli, M., et al. (2007). A functional in vitro model of rat blood-brain barrier for molecular analysis of efflux transporters. Brain Res 1150, 1-13. 
Petri, B., Kaur, J., Long, E.M., Li, H., Parsons, S.A., Butz, S., Phillipson, M., Vestweber, D., Patel, K.D., Robbins, S.M., et al. (2011). Endothelial LSP1 is involved in endothelial dome formation, minimizing vascular permeability changes during neutrophil transmigration in vivo. Blood 117, 942-952.

Phuan, P.W., Ratelade, J., Rossi, A., Tradtrantip, L., and Verkman, A.S. (2012). Complementdependent cytotoxicity in neuromyelitis optica requires aquaporin-4 protein assembly in orthogonal arrays. J Biol Chem 287, 13829-13839.

Piccio, L., Rossi, B., Scarpini, E., Laudanna, C., Giagulli, C., Issekutz, A.C., Vestweber, D., Butcher, E.C., and Constantin, G. (2002). Molecular mechanisms involved in lymphocyte recruitment in inflamed brain microvessels: critical roles for P-selectin glycoprotein ligand-1 and heterotrimeric $\mathrm{G}(\mathrm{i})$-linked receptors. J Immunol 168, 1940-1949.

Pittock, S.J., Lennon, V.A., Krecke, K., Wingerchuk, D.M., Lucchinetti, C.F., and Weinshenker, B.G. (2006). Brain abnormalities in neuromyelitis optica. Arch Neurol 63, 390-396.

Pittock, S.J., Lennon, V.A., McKeon, A., Mandrekar, J., Weinshenker, B.G., Lucchinetti, C.F., O'Toole, O., and Wingerchuk, D.M. (2013). Eculizumab in AQP4-IgG-positive relapsing neuromyelitis optica spectrum disorders: an open-label pilot study. Lancet Neurol 12, 554-562.

Plumb, J., McQuaid, S., Mirakhur, M., and Kirk, J. (2002). Abnormal endothelial tight junctions in active lesions and normal-appearing white matter in multiple sclerosis. Brain Pathol 12, 154-169.

Potschka, H., Fedrowitz, M., and Löscher, W. (2003). Multidrug Resistance Protein MRP2 Contributes to Blood-Brain Barrier Function and Restricts Antiepileptic Drug Activity. Journal of Pharmacology and Experimental Therapeutics 306, 124-131.

Proescholdt, M.A., Jacobson, S., Tresser, N., Oldfield, E.H., and Merrill, M.J. (2002). Vascular endothelial growth factor is expressed in multiple sclerosis plaques and can induce inflammatory lesions in experimental allergic encephalomyelitis rats. J Neuropathol Exp Neurol 61, 914-925.

Raab, S., Beck, H., Gaumann, A., Yuce, A., Gerber, H.P., Plate, K., Hammes, H.P., Ferrara, N., and Breier, G. (2004). Impaired brain angiogenesis and neuronal apoptosis induced by conditional homozygous inactivation of vascular endothelial growth factor. Thromb Haemost 91, 595-605.

Raine, C.S., Cannella, B., Duijvestijn, A.M., and Cross, A.H. (1990). Homing to central nervous system vasculature by antigen-specific lymphocytes. II. Lymphocyte/endothelial cell adhesion during the initial stages of autoimmune demyelination. Lab Invest 63, 476-489.

Ratelade, J., Asavapanumas, N., Ritchie, A.M., Wemlinger, S., Bennett, J.L., and Verkman, A.S. (2013). Involvement of antibody-dependent cell-mediated cytotoxicity in inflammatory demyelination in a mouse model of neuromyelitis optica. Acta Neuropathol 126, 699-709.

Ratelade, J., Bennett, J.L., and Verkman, A.S. (2011). Intravenous Neuromyelitis Optica Autoantibody in Mice Targets Aquaporin-4 in Peripheral Organs and Area Postrema. PLos One 6, e27412.

Reese, T.S., and Karnovsky, M.J. (1967). Fine structural localization of a blood-brain barrier to exogenous peroxidase. J Cell Biol 34, 207-217.

Reijerkerk, A., Kooij, G., van der Pol, S.M., Khazen, S., Dijkstra, C.D., and de Vries, H.E. (2006). Diapedesis of monocytes is associated with MMP-mediated occludin disappearance in brain endothelial cells. FASEB J 20, 2550-2552.

Rivera, J.F., Kurtzke, J.F., Booth, V.J., and Corona, V.T.t. (2008). Characteristics of Devic's disease (neuromyelitis optica) in Mexico. J Neurol 255, 710-715. 
Roemer, S.F., Parisi, J.E., Lennon, V.A., Benarroch, E.E., Lassmann, H., Bruck, W., Mandler, R.N., Weinshenker, B.G., Pittock, S.J., Wingerchuk, D.M., et al. (2007). Pattern-specific loss of aquaporin-4 immunoreactivity distinguishes neuromyelitis optica from multiple sclerosis. Brain 130, 1194-1205.

Romanic, A.M., White, R.F., Arleth, A.J., Ohlstein, E.H., and Barone, F.C. (1998). Matrix Metalloproteinase Expression Increases After Cerebral Focal Ischemia in Rats: Inhibition of Matrix Metalloproteinase-9 Reduces Infarct Size. Stroke 29, 1020-1030.

Rosell, A., Cuadrado, E., Ortega-Aznar, A., Hernandez-Guillamon, M., Lo, E.H., and Montaner, J. (2008). MMP-9-positive neutrophil infiltration is associated to blood-brain barrier breakdown and basal lamina type IV collagen degradation during hemorrhagic transformation after human ischemic stroke. Stroke 39, 1121-1126.

Rosell, A., Ortega-Aznar, A., Alvarez-Sabín, J., Fernández-Cadenas, I., Ribó, M., Molina, C.A., Lo, E.H., and Montaner, J. (2006). Increased Brain Expression of Matrix Metalloproteinase-9 After Ischemic and Hemorrhagic Human Stroke. Stroke 37, 1399-1406.

Rosenberg, G.A., Estrada, E.Y., and Dencoff, J.E. (1998). Matrix metalloproteinases and TIMPs are associated with blood-brain barrier opening after reperfusion in rat brain. Stroke 29, 2189-2195.

Rosenstein, J.M. (1987). Neocortical transplants in the mammalian brain lack a blood-brain barrier to macromolecules. Science $235,772-774$.

Saadoun, S., Waters, P., Bell, B., Vincent, A., Verkman, A., and Papadopoulos, M. (2010). Intracerebral injection of neuromyelitis optica immunoglobulin $\mathrm{G}$ and human complement produces neuromyelitis optica lesions in mice. Brain 133, 349 - 361.

Saadoun, S., Waters, P., MacDonald, C., Bell, B., Vincent, A., Verkman, A., and Papadopoulos, M. (2012). Neutrophil protease inhibition reduces NMO-IgG-induced damage in mouse brain. Ann Neurol 71, $323-333$.

Saadoun, S., Waters, P., MacDonald, C., Bridges, L.R., Bell, B.A., Vincent, A., Verkman, A.S., and Papadopoulos, M.C. (2011). T cell deficiency does not reduce lesions in mice produced by intracerebral injection of NMO-IgG and complement. Journal of Neuroimmunology 235, 27-32.

Saitou, M., Furuse, M., Sasaki, H., Schulzke, J.D., Fromm, M., Takano, H., Noda, T., and Tsukita, S. (2000). Complex phenotype of mice lacking occludin, a component of tight junction strands. Mol Biol Cell 11, 4131-4142.

Sakakibara, A., Furuse, M., Saitou, M., Ando-Akatsuka, Y., and Tsukita, S. (1997). Possible involvement of phosphorylation of occludin in tight junction formation. J Cell Biol 137, 1393-1401.

Schiera, G., Bono, E., Raffa, M.P., Gallo, A., Pitarresi, G.L., Di Liegro, I., and Savettieri, G. (2003). Synergistic effects of neurons and astrocytes on the differentiation of brain capillary endothelial cells in culture. J Cell Mol Med 7, 165-170.

Schnell, L., Fearn, S., Schwab, M.E., Perry, V.H., and Anthony, D.C. (1999). Cytokine-induced Acute Inflammation in the Brain and Spinal Cord. Journal of Neuropathology \& Experimental Neurology 58, 245-254.

Schulze, C., and Firth, J.A. (1993). Immunohistochemical localization of adherens junction components in blood-brain barrier microvessels of the rat. J Cell Sci 104 ( Pt 3), 773-782.

Seeldrayers, P.A., Syha, J., Morrissey, S.P., Stodal, H., Vass, K., Jung, S., Gneiting, T., Lassmann, H., Haase, A., Hartung, H.P., et al. (1993). Magnetic resonance imaging investigation of blood-brain 
barrier damage in adoptive transfer experimental autoimmune encephalomyelitis. J Neuroimmunol 46, 199-206.

Sekiya, S., Gotoh, S., Yamashita, T., Watanabe, T., Saitoh, S., and Sendo, F. (1989). Selective depletion of rat neutrophils by in vivo administration of a monoclonal antibody. J Leukoc Biol 46, 96-102.

Sengeløv, H., Kjeldsen, L., Kroeze, W., Berger, M., and Borregaard, N. (1994). Secretory vesicles are the intracellular reservoir of complement receptor 1 in human neutrophils. The Journal of Immunology 153, 804-810.

Shepro, D., and Morel, N.M. (1993). Pericyte physiology. FASEB J 7, 1031-1038.

Shimizu, F., Nishihara, H., Sano, Y., Takeshita, Y., Takahashi, S., Maeda, T., Takahashi, T., Abe, M., Koga, M., and Kanda, T. (2015). Markedly Increased IP-10 Production by Blood-Brain Barrier in Neuromyelitis Optica. PLos One 10, e0122000.

Shimizu, F., Sano, Y., Takahashi, T., Haruki, H., Saito, K., Koga, M., and Kanda, T. (2012). Sera from neuromyelitis optica patients disrupt the blood-brain barrier. J Neurol Neurosurg Psychiatry 83, 288297.

Simon, D.B., Lu, Y., Choate, K.A., Velazquez, H., Al-Sabban, E., Praga, M., Casari, G., Bettinelli, A., Colussi, G., Rodriguez-Soriano, J., et al. (1999). Paracellin-1, a renal tight junction protein required for paracellular Mg2+ resorption. Science 285, 103-106.

Snipes, M.B., Barnett, A.L., Harkema, J.R., Hotchkiss, J.A., Rebar, A.H., and Reddick, L.J. (1995). Specific Biological Effects of an Anti-Rat PMN Antiserum Intraperitoneally Injected into F344/N Rats. Veterinary Clinical Pathology 24, 11-17.

Snyderman, R., and Pike, M.C. (1984). Chemoattractant receptors on phagocytic cells. Annu Rev Immunol 2, 257-281.

Soehnlein, O., Zernecke, A., Eriksson, E.E., Rothfuchs, A.G., Pham, C.T., Herwald, H., Bidzhekov, K., Rottenberg, M.E., Weber, C., and Lindbom, L. (2008). Neutrophil secretion products pave the way for inflammatory monocytes. Blood 112, 1461-1471.

Stamatovic, S.M., Shakui, P., Keep, R.F., Moore, B.B., Kunkel, S.L., Van Rooijen, N., and Andjelkovic, A.V. (2005). Monocyte chemoattractant protein-1 regulation of blood-brain barrier permeability. J Cereb Blood Flow Metab 25, 593-606.

Stanimirovic, D., Shapiro, A., Wong, J., Hutchison, J., and Durkin, J. (1997). The induction of ICAM-1 in human cerebromicrovascular endothelial cells (HCEC) by ischemia-like conditions promotes enhanced neutrophil/HCEC adhesion. Journal of Neuroimmunology 76, 193-205.

Stark, M.A., Huo, Y., Burcin, T.L., Morris, M.A., Olson, T.S., and Ley, K. (2005). Phagocytosis of apoptotic neutrophils regulates granulopoiesis via IL-23 and IL-17. Immunity 22, 285-294.

Steed, E., Rodrigues, N., Balda, M., and Matter, K. (2009). Identification of MarvelD3 as a tight junction-associated transmembrane protein of the occludin family. BMC Cell Biol 10, 1-14.

Stenman, J.M., Rajagopal, J., Carroll, T.J., Ishibashi, M., McMahon, J., and McMahon, A.P. (2008). Canonical Wnt signaling regulates organ-specific assembly and differentiation of CNS vasculature. Science 322, 1247-1250. 
Stevenson, B.R., Siliciano, J.D., Mooseker, M.S., and Goodenough, D.A. (1986). Identification of ZO-1: a high molecular weight polypeptide associated with the tight junction (zonula occludens) in a variety of epithelia. The Journal of Cell Biology 103, 755-766.

Stewart, P.A., and Wiley, M.J. (1981). Developing nervous tissue induces formation of blood-brain barrier characteristics in invading endothelial cells: a study using quail--chick transplantation chimeras. Dev Biol 84, 183-192.

Strong, L.H. (1964). The early embryonic pattern of internal vascularization of the mammalian cerebral cortex. The Journal of Comparative Neurology 123, 121-138.

Subileau, E.A., Rezaie, P., Davies, H.A., Colyer, F.M., Greenwood, J., Male, D.K., and Romero, I.A. (2009). Expression of chemokines and their receptors by human brain endothelium: implications for multiple sclerosis. J Neuropathol Exp Neurol 68, 227-240.

Suehiro, E., Fujisawa, H., Akimura, T., Ishihara, H., Kajiwara, K., Kato, S., Fujii, M., Yamashita, S., Maekawa, T., and Suzuki, M. (2004). Increased Matrix Metalloproteinase-9 in Blood in Association with Activation of Interleukin-6 after Traumatic Brain Injury: Influence of Hypothermic Therapy. Journal of Neurotrauma 21, 1706-1711.

Suzuki, A., Ishiyama, C., Hashiba, K., Shimizu, M., Ebnet, K., and Ohno, S. (2002). aPKC kinase activity is required for the asymmetric differentiation of the premature junctional complex during epithelial cell polarization. J Cell Sci 115, 3565-3573.

Suzuki, N., Ishii, Y., and Kitamura, S. (1994). Mechanism for the Increased Permeability in Endothelial Monolayers Induced by Elastase. Mediators Inflamm 3, 11-16.

Takahashi, T., Fujihara, K., Nakashima, I., Misu, T., Miyazawa, I., Nakamura, M., Watanabe, S., Shiga, Y., Kanaoka, C., Fujimori, J., et al. (2007). Anti-aquaporin-4 antibody is involved in the pathogenesis of NMO: a study on antibody titre. Brain 130, 1235-1243.

Takenaga, Y., Takagi, N., Murotomi, K., Tanonaka, K., and Takeo, S. (2009). Inhibition of Src activity decreases tyrosine phosphorylation of occludin in brain capillaries and attenuates increase in permeability of the blood-brain barrier after transient focal cerebral ischemia. J Cereb Blood Flow Metab 29, 1099-1108.

Tasaki, A., Shimizu, F., Sano, Y., Fujisawa, M., Takahashi, T., Haruki, H., Abe, M., Koga, M., and Kanda, T. (2014). Autocrine MMP-2/9 secretion increases the BBB permeability in neuromyelitis optica. J Neurol Neurosurg Psychiatry 85, 419-430.

Tian, J., Avalos, A.M., Mao, S.Y., Chen, B., Senthil, K., Wu, H., Parroche, P., Drabic, S., Golenbock, D., Sirois, C., et al. (2007). Toll-like receptor 9-dependent activation by DNA-containing immune complexes is mediated by HMGB1 and RAGE. Nat Immunol 8, 487-496.

Tomizawa, Y., Yokoyama, K., Saiki, S., Takahashi, T., Matsuoka, J., and Hattori, N. (2012). Blood-brain barrier disruption is more severe in neuromyelitis optica than in multiple sclerosis and correlates with clinical disability. J Int Med Res 40, 1483-1491.

Tonai, T., Shiba, K.-i., Taketani, Y., Ohmoto, Y., Murata, K., Muraguchi, M., Ohsaki, H., Takeda, E., and Nishisho, T. (2001). A neutrophil elastase inhibitor (ONO-5046) reduces neurologic damage after spinal cord injury in rats. Journal of Neurochemistry 78, 1064-1072.

Tradtrantip, L., Zhang, H., Saadoun, S., Phuan, P.W., Lam, C., Papadopoulos, M.C., Bennett, J.L., and Verkman, A.S. (2012). Anti-aquaporin-4 monoclonal antibody blocker therapy for neuromyelitis optica. Ann Neurol 71, 314-322. 
Tsukamoto, T., and Nigam, S.K. (1999). Role of tyrosine phosphorylation in the reassembly of occludin and other tight junction proteins. Am J Physiol 276, F737-750.

Turowski, P., Martinelli, R., Crawford, R., Wateridge, D., Papageorgiou, A.P., Lampugnani, M.G., Gamp, A.C., Vestweber, D., Adamson, P., Dejana, E., et al. (2008). Phosphorylation of vascular endothelial cadherin controls lymphocyte emigration. J Cell Sci 121, 29-37.

Van Itallie, C.M., Fanning, A.S., Holmes, J., and Anderson, J.M. (2010). Occludin is required for cytokine-induced regulation of tight junction barriers. Journal of Cell Science 123, 2844-2852.

Vincent, T., Saikali, P., Cayrol, R., Roth, A.D., Bar-Or, A., Prat, A., and Antel, J.P. (2008). Functional Consequences of Neuromyelitis Optica-IgG Astrocyte Interactions on Blood-Brain Barrier Permeability and Granulocyte Recruitment. The Journal of Immunology, 5730-5737.

Virapongse, C., Mancuso, A., and Quisling, R. (1986). Human brain infarcts: Gd-DTPA-enhanced MR imaging. Radiology 161, 785-794.

Visser, C.C., Voorwinden, L.H., Crommelin, D.J., Danhof, M., and de Boer, A.G. (2004). Characterization and modulation of the transferrin receptor on brain capillary endothelial cells. Pharm Res 21, 761-769.

von Wedel-Parlow, M., Schrot, S., Lemmen, J., Treeratanapiboon, L., Wegener, J., and Galla, H.J. (2011). Neutrophils cross the BBB primarily on transcellular pathways: an in vitro study. Brain Res $1367,62-76$.

Wachtel, M., Frei, K., Ehler, E., Fontana, A., Winterhalter, K., and Gloor, S.M. (1999). Occludin proteolysis and increased permeability in endothelial cells through tyrosine phosphatase inhibition. $J$ Cell Sci 112 ( Pt 23), 4347-4356.

Watanabe, S., Nakashima, I., Misu, T., Miyazawa, I., Shiga, Y., Füjihara, K., and Itoyama, Y. (2007). Therapeutic efficacy of plasma exchange in NMO-IgG-positive patients with neuromyelitis optica. Multiple Sclerosis 13, 128-132.

Waters, P., McKeon, A., Leite, M., Rajasekharan, S., Lennon, V., Villalobos, A., Palace, J., Mandrekar, J., Vincent, A., Bar-Or, A., et al. (2012). Serologic diagnosis of NMO: a multicenter comparison of aquaporin-4-IgG assays. Neurology 78, 665 - 671.

Willis, C.L., Camire, R.B., Brule, S.A., and Ray, D.E. (2013). Partial recovery of the damaged rat bloodbrain barrier is mediated by adherens junction complexes, extracellular matrix remodeling and macrophage infiltration following focal astrocyte loss. Neuroscience $250,773-785$.

Willis, C.L., Nolan, C.C., Reith, S.N., Lister, T., Prior, M.J.W., Guerin, C.J., Mavroudis, G., and Ray, D.E. (2004). Focal astrocyte loss is followed by microvascular damage, with subsequent repair of the blood-brain barrier in the apparent absence of direct astrocytic contact. Glia 45, 325-337.

Wingerchuk, D.M., Hogancamp, W.F., O'Brien, P.C., and Weinshenker, B.G. (1999). The clinical course of neuromyelitis optica (Devic's syndrome). Neurology 53, 1107-1114.

Wingerchuk, D.M., Lennon, V.A., Lucchinetti, C.F., Pittock, S.J., and Weinshenker, B.G. (2007). The spectrum of neuromyelitis optica. The Lancet Neurology 6, 805-815.

Wolburg, H., Noell, S., Mack, A., Wolburg-Buchholz, K., and Fallier-Becker, P. (2008). Brain endothelial cells and the glio-vascular complex. Cell and Tissue Research 335, 75-96. 
Wolburg, H., Wolburg-Buchholz, K., and Engelhardt, B. (2005). Diapedesis of mononuclear cells across cerebral venules during experimental autoimmune encephalomyelitis leaves tight junctions intact. Acta Neuropathol 109, 181-190.

Wolburg, H., Wolburg-Buchholz, K., Kraus, J., Rascher-Eggstein, G., Liebner, S., Hamm, S., Duffner, F., Grote, E.H., Risau, W., and Engelhardt, B. (2003). Localization of claudin-3 in tight junctions of the blood-brain barrier is selectively lost during experimental autoimmune encephalomyelitis and human glioblastoma multiforme. Acta Neuropathol 105, 586-592.

Wong, A.K., Finch, A.M., Pierens, G.K., Craik, D.J., Taylor, S.M., and Fairlie, D.P. (1998). Small molecular probes for G-protein-coupled C5a receptors: conformationally constrained antagonists derived from the $C$ terminus of the human plasma protein C5a. J Med Chem 41, 3417-3425.

Wong, D., Prameya, R., and Dorovini-Zis, K. (2007). Adhesion and migration of polymorphonuclear leukocytes across human brain microvessel endothelial cells are differentially regulated by endothelial cell adhesion molecules and modulate monolayer permeability. J Neuroimmunol 184, 136-148.

Wong, V. (1997). Phosphorylation of occludin correlates with occludin localization and function at the tight junction. Am J Physiol 273, C1859-1867.

Woodruff, T.M., Strachan, A.J., Dryburgh, N., Shiels, I.A., Reid, R.C., Fairlie, D.P., and Taylor, S.M. (2002). Antiarthritic activity of an orally active C5a receptor antagonist against antigen-induced monarticular arthritis in the rat. Arthritis \& Rheumatism 46, 2476-2485.

Wosik, K., Biernacki, K., Khouzam, M.P., and Prat, A. (2007a). Death receptor expression and function at the human blood brain barrier. J Neurol Sci 259, 53-60.

Wosik, K., Cayrol, R., Dodelet-Devillers, A., Berthelet, F., Bernard, M., Moumdjian, R., Bouthillier, A., Reudelhuber, T.L., and Prat, A. (2007b). Angiotensin II controls occludin function and is required for blood brain barrier maintenance: relevance to multiple sclerosis. J Neurosci 27, 9032-9042.

Wrzos, C., Winkler, A., Metz, I., Kayser, D.M., Thal, D.R., Wegner, C., Bruck, W., Nessler, S., Bennett, J.L., and Stadelmann, C. (2014). Early loss of oligodendrocytes in human and experimental neuromyelitis optica lesions. Acta Neuropathol 127, 523-538.

Yagisawa, M., Yuo, A., Kitagawa, S., Yazaki, Y., Togawa, A., and Takaku, F. (1995). Stimulation and priming of human neutrophils by IL-1 alpha and IL-1 beta: complete inhibition by IL-1 receptor antagonist and no interaction with other cytokines. Exp Hematol 23, 603-608.

Yamamoto, S., Shimizu, S., Kiyonaka, S., Takahashi, N., Wajima, T., Hara, Y., Negoro, T., Hiroi, T., Kiuchi, Y., Okada, T., et al. (2008). TRPM2-mediated Ca2+influx induces chemokine production in monocytes that aggravates inflammatory neutrophil infiltration. Nat Med 14, 738-747.

Yamanaka, T., Horikoshi, Y., Suzuki, A., Sugiyama, Y., Kitamura, K., Maniwa, R., Nagai, Y., Yamashita, A., Hirose, T., Ishikawa, H., et al. (2001). PAR-6 regulates aPKC activity in a novel way and mediates cell-cell contact-induced formation of the epithelial junctional complex. Genes Cells 6, 721-731.

Yamasaki, S., Ishikawa, E., Sakuma, M., Hara, H., Ogata, K., and Saito, T. (2008). Mincle is an ITAMcoupled activating receptor that senses damaged cells. Nat Immunol 9, 1179-1188.

Yanai, H., Ban, T., Wang, Z., Choi, M.K., Kawamura, T., Negishi, H., Nakasato, M., Lu, Y., Hangai, S., Koshiba, R., et al. (2009). HMGB proteins function as universal sentinels for nucleic-acid-mediated innate immune responses. Nature 462, 99-103. 
Yang, L., Froio, R.M., Sciuto, T.E., Dvorak, A.M., Alon, R., and Luscinskas, F.W. (2005). ICAM-1 regulates neutrophil adhesion and transcellular migration of TNF-alpha-activated vascular endothelium under flow. Blood 106, 584-592.

Yang, Y., Estrada, E.Y., Thompson, J.F., Liu, W., and Rosenberg, G.A. (2007). Matrix metalloproteinasemediated disruption of tight junction proteins in cerebral vessels is reversed by synthetic matrix metalloproteinase inhibitor in focal ischemia in rat. J Cereb Blood Flow Metab 27, 697-709.

Zhang, H., Bennett, J.L., and Verkman, A.S. (2011). Ex vivo spinal cord slice model of neuromyelitis optica reveals novel immunopathogenic mechanisms. Annals of Neurology 70, 943-954.

Zhang, H., and Verkman, A.S. (2013). Eosinophil pathogenicity mechanisms and therapeutics in neuromyelitis optica. J Clin Invest 123, 2306-2316.

Zhang, Y., Han, H., Elmquist, W.F., and Miller, D.W. (2000). Expression of various multidrug resistance-associated protein (MRP) homologues in brain microvessel endothelial cells. Brain Research 876, 148-153.

Zlokovic, B.V., Skundric, D.S., Segal, M.B., Lipovac, M.N., Mackic, J.B., and Davson, H. (1990). A saturable mechanism for transport of immunoglobulin $\mathrm{G}$ across the blood-brain barrier of the guinea pig.

Exp

Neurol

107

263-270. 
ABBREVIATIONS 